\title{
Molecular response properties from a Hermitian eigenvalue equation for a time- periodic Hamiltonian
}

Filip Pawłowski ${ }^{\prime}$, Jeppe Olsen, and Poul Jørgensen

Citation: The Journal of Chemical Physics 142, 114109 (2015); doi: 10.1063/1.4913364

View online: http://dx.doi.org/10.1063/1.4913364

View Table of Contents: http://aip.scitation.org/toc/jcp/142/11

Published by the American Institute of Physics

\section{Articles you may be interested in}

Molecular response properties in equation of motion coupled cluster theory: A time-dependent perspective The Journal of Chemical Physics 144, 024102 (2016); 10.1063/1.4939183

Perspective: Explicitly correlated electronic structure theory for complex systems

The Journal of Chemical Physics 146, 080901 (2017); 10.1063/1.4976974

Linear and nonlinear response functions for an exact state and for an MCSCF state

The Journal of Chemical Physics 82, 3235 (1998); 10.1063/1.448223

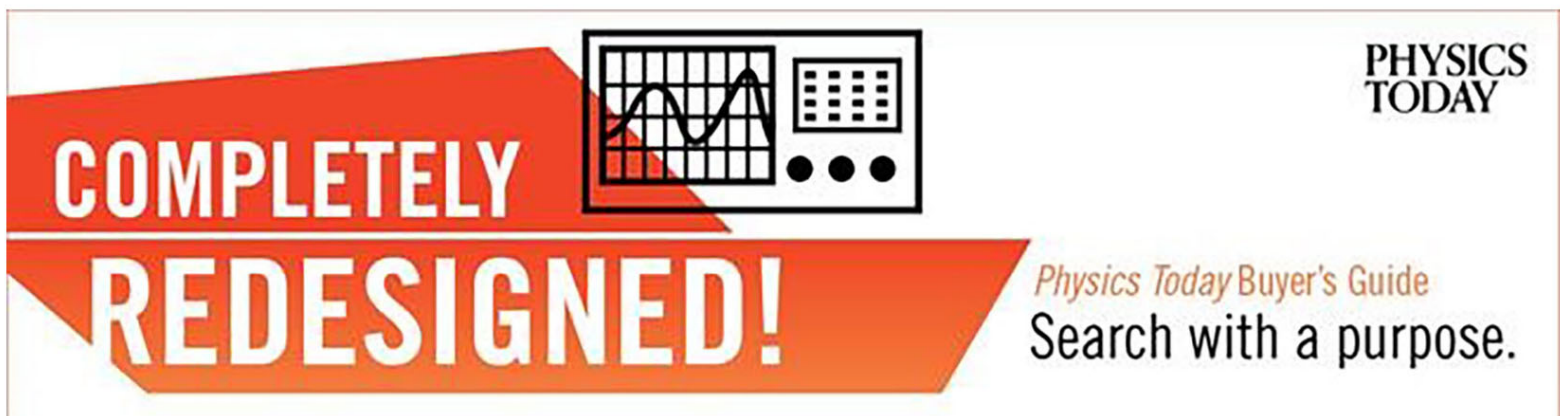




\title{
Molecular response properties from a Hermitian eigenvalue equation for a time-periodic Hamiltonian
}

\author{
Filip Pawłowski, ${ }^{1,2, a)}$ Jeppe Olsen, ${ }^{1}$ and Poul Jørgensen ${ }^{1}$ \\ ${ }^{1}$ qLEAP Center for Theoretical Chemistry, Department of Chemistry, Aarhus University, Langelandsgade 140, \\ DK-8000 Aarhus C, Denmark \\ ${ }^{2}$ Institute of Physics, Kazimierz Wielki University, Plac Weyssenhoffa 11, 85-072 Bydgoszcz, Poland
}

(Received 23 December 2014; accepted 11 February 2015; published online 18 March 2015)

\begin{abstract}
The time-dependent Schrödinger equation for a time-periodic perturbation is recasted into a Hermitian eigenvalue equation, where the quasi-energy is an eigenvalue and the time-periodic regular wave function an eigenstate. From this Hermitian eigenvalue equation, a rigorous and transparent formulation of response function theory is developed where (i) molecular properties are defined as derivatives of the quasi-energy with respect to perturbation strengths, (ii) the quasi-energy can be determined from the time-periodic regular wave function using a variational principle or via projection, and (iii) the parametrization of the unperturbed state can differ from the parametrization of the time evolution of this state. This development brings the definition of molecular properties and their determination on par for static and time-periodic perturbations and removes inaccuracies and inconsistencies of previous response function theory formulations. The development where the parametrization of the unperturbed state and its time evolution may differ also extends the range of the wave function models for which response functions can be determined. The simplicity and universality of the presented formulation is illustrated by applying it to the configuration interaction (CI) and the coupled cluster (CC) wave function models and by introducing a new model-the coupled cluster configuration interaction (CC-CI) model-where a coupled cluster exponential parametrization is used for the unperturbed state and a linear parametrization for its time evolution. For static perturbations, the CC-CI response functions are shown to be the analytical analogues of the static molecular properties obtained from finite field equation-of-motion coupled cluster (EOMCC) energy calculations. The structural similarities and differences between the CI, CC, and CC-CI response functions are also discussed with emphasis on linear versus non-linear parametrizations and the size-extensivity of the obtained molecular properties. C 2015 AIP Publishing LLC. [http://dx.doi.org/10.1063/1.4913364]
\end{abstract}

\section{INTRODUCTION}

Response functions provide a framework which allows the determination of molecular properties for a variety of quantum chemical models. We present a transparent and rigorous formulation of response function theory, where the application range of response theory is extended and where the derivation follows a different and simpler route compared to previous formulations. ${ }^{1,2}$ The cornerstone is the recasting of the time-dependent Schrödinger equation for a time-periodic perturbation into a Hermitian eigenvalue equation where the quasi-energy is an eigenvalue and the timeperiodic regular wave function an eigenstate. In the static limit, this Hermitian eigenvalue equation becomes the Hermitian eigenvalue equation for static perturbations. For static perturbations, molecular properties are defined as derivatives of the eigenvalue with respect to perturbation strengths. For periodic perturbations, this definition of molecular properties thus extends to derivatives of the quasi-energy.

Recasting the time-dependent Schrödinger equation for a time-periodic perturbation into a Hermitian eigenvalue

\footnotetext{
a)Electronic mail: filip.pawlowski1 @ gmail.com
}

equation is by itself not new and has been carried out, for example, in the context of Floquet theory ${ }^{3-5}$ and steady-state ${ }^{6}$ theory. In the steady-state formulation, the time-dependent wave function is written as an exponential phase-factor containing the quasi-energy times a time-dependent wave function, the steady-state wave function. Using simple timetranslational arguments, it is easy to see that the steady-state wave function is time-periodic with the same period as the Hamiltonian. However, the steady-state wave function does not provide the best starting point for the development of approximate time-dependent quantum mechanical methods. In particular, the steady-state wave function contains an overall phase, which is redundant and often leads to problems, in particular in the time-independent limit. To avoid this problem, it is useful to introduce the regular wave function defined as the steady-state wave function times a time-dependent phasefactor, which is chosen so that the time-dependent coefficient of a chosen reference state is real and positive at all times. In the development of Sambe ${ }^{6}$ it is shown that the regular wave function has the same periodicity as the steady-state wave function, provided the reference state is a solution to a timeindependent Schrödinger equation. In the present work, this restriction is removed, i.e., we allow an arbitrary normalized state with non-vanishing overlap with the steady-state wave 
function as the reference state and show that the regular and steady-state wave functions have the same periodicity.

The regular wave function provides the starting point for developing approximate time-dependent methods free of singularities for the wave functions that are normalized at all times. For a number of standard approximate wave function models, in particular coupled cluster (CC) methods, an intermediate normalized rather than a unit-norm normalized wave function is used. By eliminating the amplitude of the reference wave function from the regular wave function, we show that the regular wave function is then also time-periodic and fulfills a slightly modified eigenvalue equation than for a unit-normalized state.

We have thus described a direct route where, using the time-translational invariance of the Hamiltonian, the time-dependent Schrödinger equation can be recasted into a Hermitian eigenvalue equation and where molecular response functions are defined as derivatives of the quasi-energy with respect to the perturbation strengths. The advantages of defining molecular properties in this way compared to the traditional one, where response functions are defined in terms of the expansion coefficients of a perturbation expansion of an expectation value for an observable, are also discussed.

The formulation we present allows for a different parametrization of the unperturbed state and its time evolution. This makes it possible to derive response functions for new classes of states. For example, we introduce a new model with a coupled cluster unperturbed state and a linear parametrization of the time evolution, which we denote the coupled cluster configuration interaction (CC-CI) model, and derive response functions for this model. In the static limit, molecular properties obtained with the CC-CI model become an analytic representation of the static molecular properties obtained from finite field equation-of-motion coupled cluster (EOMCC) energy calculations. The CC-CI molecular properties are not size-extensive; however, we show that the lack of sizeextensivity is weak as the unperturbed system is described by a coupled cluster state. We also derive response functions for the standard configuration interaction (CI) and CC wave function models and discuss the size-extensivity of molecular properties for these models for truncated excitation manifolds.

The formulation we present simplifies the derivation of response functions and allows us to solve inadequacies and inconsistencies of previous formulations of response function theory. ${ }^{1,2}$ For example, in the previous formulations of response function theory, time evolution has only been considered for states with a norm fixed in time and results were assumed to carry over for states with time-evolving norms, as coupled cluster states. The formulation we develop here is rigorously applicable to states with both a constant and a time-evolving norm.

Response function theory was introduced in the physics literature in the 1950s and 1960s. We refer to the books of Zubarev $^{7}$ and Linderberg and Öhrn ${ }^{8}$ for more details. Response function theory was introduced into chemistry in the 1960s and $1970 \mathrm{~s}^{9-11}$ and in its contemporary form formulated in 1985 by Olsen and Jørgensen ${ }^{1}$ for determining molecular properties for both ground and excited states and transition properties between these states. Olsen and Jørgensen ${ }^{1}$ derived response functions for exact and multi-configurational selfconsistent field (MCSCF) reference states. The response functions were identified from a perturbation expansion of the expectation value of an observable, and the permutational symmetry of response functions with respect to interchange of operator-frequency pairs was for that reason far from trivial to obtain within this formulation. ${ }^{12}$

The first-generation formulation of response function theory was followed by implementations of MCSCF linear, ${ }^{13}$ quadratic, ${ }^{14,15}$ and cubic $^{16,17}$ response functions and their residues and numerous applications of MCSCF response theory in various fields of chemistry and physics have been reported, see, for example, Refs. 18-31.

The derivation of response functions from a perturbation expansion of the expectation value of an observable was in 1990 generalized by Koch and Jørgensen ${ }^{32}$ to comprehend coupled cluster wave functions and implementations were reported for a coupled cluster singles and doubles (CCSD) state for excitation energies, ${ }^{33}$ transition strengths, ${ }^{34}$ and polarizabilities. ${ }^{35}$

In 1998, Christiansen, Hättig, and Jørgensen published the second generation formulation of response function theory ${ }^{2}$ that treated variational and non-variational states in a uniform manner. Central for that formulation was the generalization of the energy of time-independent theory to the quasi-energy for time-periodic perturbations and the recognition that the quasienergy satisfies, as the energy, a variational principle. Using the generalized Hellmann-Feynman theorem, the response functions were shown, in the spirit of an earlier work by Aiga, Sasagane, and Itoh, ${ }^{36,37}$ to be derivatives of the quasi-energy with respect to the perturbation strengths. For non-variational states, the quasi-energy was replaced by the quasi-energy Lagrangian and the time evolution of a non-variational state was determined from the stationary conditions for the quasienergy Lagrangian. Response functions for non-variational states were determined as derivatives of the quasi-energy Lagrangian. Expressing response functions as quasi-energy or quasi-energy Lagrangian derivatives automatically yielded the operator-frequency pair permutational symmetries of the response functions, which were cumbersome to identify in the first generation formulation of response function theory. The second generation formulation also allowed the application of the $2 n+1$ and $2 n+2$ rules, ${ }^{38-41}$ thereby considerably simplifying response functions expressions.

Identifying the response functions as quasi-energy Lagrangian derivatives allowed also the derivation of response functions for approximate coupled cluster models, where simplifications were introduced in the coupled cluster amplitude equations based, for example, on a Møller-Plesset partitioning of the Hamiltonian. This led to the coupled cluster response hierarchy $\mathrm{CCS}, \mathrm{CC} 2,{ }^{42} \mathrm{CCSD},{ }^{43} \mathrm{CC} 3,{ }^{44-46}$ CCSDT,${ }^{47,48} \ldots$ and also to response functions for other iterative approximate coupled cluster wave function models. ${ }^{49-54}$

The second generation formulation boosted efficient implementations of response functions for coupled cluster and approximate coupled cluster wave function models that have been widely used. ${ }^{55-66}$ Further, the second generation formulation of response function theory allowed for the development of linear-scaling implementations and applications of 
density-matrix based quasi-energy Hartree-Fock and KohnSham response theories. ${ }^{67-69}$ Damped response function theory was introduced by Norman et al. , $^{70,71}$ where finite excitedstate lifetimes were introduced as an alternative to a residue analysis for addressing resonance spectroscopies. ${ }^{72-75}$ For a comprehensive review of the developments, implementations, and applications of response function theory until 2012, see also Ref. 76. The second generation formulation thus advanced response function theory to become a commonly used black box tool for predicting molecular properties for ground and excited states and for transitions between these states.

Despite its great success, the second generation formulation suffered from several formal deficiencies. One example is the legitimacy of generalizing the determination of response functions as derivatives of the quasi-energy to the quasi-energy Lagrangian. The quasi-energy is obtained solving the timedependent Schrödinger equation for a time-evolving state with a unit norm, an assumption that clearly cannot be applied for non-variational wave functions that have a time-evolving norm, for example, a coupled cluster wave function. Another example is that for approximate non-variational states, the Hermiticity of an expectation value of an observable is not guaranteed. This deficiency was circumvented by an $a d$ hoc introduction of an operator that symmetrizes response functions with respect to simultaneous sign inversion of the frequencies and complex conjugation, thus superimposing the symmetry relation of the response functions for an exact state on the response functions for approximate non-variational states, for example, coupled cluster states.

The formulation presented in this article leads to molecular response properties expressed as derivatives of the quasienergy or the quasi-energy Lagrangian, as did the second generation formulation of 1998, but without the inadequacies of the previous formulations. The way in which we arrive at the quasi-energy and quasi-energy Lagrangian is, however, very different. In our formulation, the quasi-energy is an eigenvalue of a Hermitian eigenvalue equation and molecular properties may therefore straightforwardly be defined by differentiation of the eigenvalue with respect to perturbation strengths. This direct way of defining molecular response properties does not require any recourse to the generalized HellmannFeynman theorem. The operator that symmetrizes response functions with respect to simultaneous sign inversion of the frequencies and complex conjugation arises automatically in the formulation presented here.

Another advantage of the formulation presented here is that the parametrization of the time evolution is separated from the parametrization of the unperturbed state. This allows us to eliminate the zero-order amplitude and multiplier equations from the quasi-energy Lagrangian at an early stage of the derivation, which greatly simplifies the derivation of response functions, compared to previous formulations. Additional simplifications result from the fact that we may use the $2 n+1$ and $2 n+2$ rules, as in the formulation of 1998 in order to derive the response functions. However, we may also use the generalizations of these rules, as formulated by Kristensen et al. ${ }^{77}$

The simplicity in our derivation of response functions is illustrated by deriving linear, quadratic, and cubic response functions for $\mathrm{CI}, \mathrm{CC}-\mathrm{CI}$, and $\mathrm{CC}$ wave function models. The CI, CC-CI, and CC models constitute different parametrizations of the same exact solution to the time-dependent Schrödinger equation and, therefore, provide full configuration interaction (FCI) results in the limit, where no truncations are performed in the excitation manifold. In both the CI and the CC-CI models, the time evolution is linearly parametrized, in CI combined with a time-independent CI reference state, while in CC-CI with a time-independent $\mathrm{CC}$ reference state. The linear parametrization of the time evolution makes it possible to obtain an explicit representation of the ground and excited states, but this happens at the expense that the $\mathrm{CI}$ and CC-CI molecular properties are not size-extensive. In the $\mathrm{CC}$ model, both the time-independent reference state and the time evolution are exponentially parametrized. The exponential parametrization of the time evolution makes it impossible to obtain an explicit representation of the ground and excited states. This, however, does not pose any problem, since explicit expressions for molecular properties may be obtained from the response functions. In turn, the exponential parametrization ensures that the $\mathrm{CC}$ molecular properties are size-extensive, also when truncations are performed in the excitation manifold.

We also compare the CC-CI response functions with the EOMCC response functions. EOMCC response functions are not derived by differentiation of a quasi-energy Lagrangian but, as described by Stanton and Bartlett ${ }^{78}$ and Rozyczko and Bartlett, ${ }^{79-81}$ from CI response functions by substituting the $\mathrm{CI}$ energies, states, and response vectors with their EOMCC counterparts. In this article, we show that the EOMCC and CC-CI linear response functions are identical. We show also that the EOMCC and CC-CI quadratic (and higher-order) response functions differ and that the EOMCC quadratic, cubic, etc., response functions therefore cannot reproduce the FCI limit results.

In Sec. II, we recast the time-dependent Schrödinger equation for a time-periodic perturbation into a Hermitian eigenvalue equation. We eliminate the time-dependent phase from the eigenstate and show that in the time-independent limit the phase-isolated eigenstate (the regular wave function) becomes the eigenstate and the quasi-energy the eigenvalue of the time-independent Hamiltonian. Molecular response properties for a time-periodic Hamiltonian may therefore be defined as derivatives of the quasi-energy with respect to timeperiodic perturbation strengths, in the same way the static molecular properties are defined as derivatives of the energy with respect to static perturbation strengths. In Sec. III we show how molecular response functions may be obtained when the Hermitian eigenvalue equation is solved using a variational principle. We also compare the definition of response functions as derivatives of the quasi-energy to the standard definition as the time-dependent expansion coefficients of the expectation value of a perturbation operator. In Sec. IV, we show how molecular response functions may be obtained as derivatives of the quasi-energy Lagrangian when the Hermitian eigenvalue equation is solved via projection. We also parametrize the time-dependent wave function such that the parametrization and optimization of the unperturbed system may differ from the parametrization and optimization of its time evolution. We 
apply the general development of Sec. IV in Secs. VI-VIII to derive response functions for the $\mathrm{CI}, \mathrm{CC}-\mathrm{CI}$, and $\mathrm{CC}$ models. The EOMCC response functions are given in Sec. IX. A comprehensive comparison between CI, CC-CI, and CC response functions is carried out in Sec. $\mathrm{X}$ with emphasis on linear versus exponential parametrization and the sizeextensivity of molecular response properties. We also show that the CC-CI static limit response properties may be obtained from EOMCC finite field energy calculations. Section XI contains concluding remarks.

\section{THE TIME-DEPENDENT SCHRÖDINGER EQUATION FOR A PERIODIC PERTURBATION AS A HERMITIAN EIGENVALUE EQUATION}

In this section, we present the foundation for recasting the time-dependent Schrödinger equation for a time-periodic Hamiltonian into a Hermitian eigenvalue equation. We also describe how molecular properties become the derivatives of the eigenvalue of this Hermitian eigenvalue equation with respect to perturbation strengths and how molecular response functions therefore may be obtained solving the Hermitian eigenvalue equation. This development brings the determination of molecular properties for time-periodic and static perturbations on par.

\section{A. The steady-state wave function and its eigenvalue equation}

Consider the time-dependent Schrödinger equation, which in atomic units becomes

$$
\left.H(t, \epsilon)|\widetilde{0}(t, \epsilon)\rangle=i \frac{\partial}{\partial t} \widetilde{0}(t, \epsilon)\right\rangle,
$$

where $H(t, \epsilon)$ is a time-dependent bounded Hermitian Hamiltonian

$$
H(t, \epsilon)=H_{0}+V(t, \epsilon)
$$

where $H_{0}$ is the time-independent Hamiltonian of the unperturbed system and $V(t, \epsilon)$ is a time-dependent perturbation. $V(t, \epsilon)$ may be expanded in a sum over Fourier components,

$$
V(t, \epsilon)=\sum_{j} X_{j} \epsilon_{X_{j}}\left(\omega_{X_{j}}\right) \exp \left(-i \omega_{X_{j}} t\right),
$$

where $X_{j}$ is a Hermitian time-independent operator and $\epsilon_{X_{j}}$ $\left(\omega_{X_{j}}\right)$ is the associated perturbation strength for the real frequency $\omega_{X_{j}}$. $\epsilon$ denotes a set of perturbation strengths $\epsilon_{X_{j}}$ $\left(\omega_{X_{j}}\right)$. To obtain a Hermitian perturbation operator, the sum in Eq. (3) includes for each term $X_{j} \epsilon_{X_{j}}\left(\omega_{X_{j}}\right) \exp \left(-i \omega_{X_{j}} t\right)$ also its adjoint $X_{j}^{\dagger} \epsilon_{X_{j}}\left(-\omega_{X_{j}}\right) \exp \left(i \omega_{X_{j}} t\right)$ with

$$
\epsilon_{X_{j}}\left(-\omega_{X_{j}}\right)=\epsilon_{X_{j}}^{*}\left(\omega_{X_{j}}\right) \text {. }
$$

We will in the following consider solely perturbations that are periodic in time with a period $T$,

$$
V(t+T, \epsilon)=V(t, \epsilon) .
$$

It then follows from Eq. (3) that associated with the period $T$, there is a fundamental frequency $\omega_{T}$,

$$
\omega_{T}=\frac{2 \pi}{T}
$$

such that the frequencies in Eq. (3) become an integer times the fundamental frequency,

$$
\omega_{X_{j}}=n_{X_{j}} \omega_{T}
$$

As the Hamiltonian, $H(t, \epsilon)$ of Eq. (2), is bounded, it has solutions with finite norm, and we consider in the following only such solutions. From the Hermiticity of the Hamiltonian, it follows that the norm of $\widetilde{0}(t, \epsilon)\rangle$ is independent of time. We choose this norm to be one,

$$
\langle\widetilde{0}(t, \epsilon) \mid \widetilde{0}(t, \epsilon)\rangle=1 .
$$

The time-periodicity of the perturbation operator in Eq. (5) implies that the Hamiltonian $H(t, \epsilon)$ of Eq. (2) is invariant with respect to time translations of the length $k T$, where $k$ is an integer,

$$
\left[\mathcal{T}_{t}(k T), H(t, \epsilon)\right]=0, \quad k=0, \pm 1, \pm 2, \ldots,
$$

where $\mathcal{T}_{t}(\Delta t)$ is a time-translation operator that carries out a translation of a state $|f(t)\rangle$ from an initial time $t$ to a final time $t+\Delta t$

$$
\mathcal{T}_{t}(\Delta t)|f(t)\rangle=|f(t+\Delta t)\rangle
$$

Acting with the time-translation operator on the Schrödinger equation [Eq. (1)], we obtain

$$
\left.\mathcal{T}_{t}(k T) H(t, \epsilon)|\widetilde{0}(t, \epsilon)\rangle=\mathcal{T}_{t}(k T) i \frac{\partial}{\partial t} \widetilde{\mid 0}(t, \epsilon)\right\rangle .
$$

Using Eq. (9), and the fact that the time differentiation operator commutes with the time-translation operator, we obtain from Eq. (11)

$$
H(t, \epsilon)\left(\mathcal{T}_{t}(k T)|\widetilde{0}(t, \epsilon)\rangle\right)=i \frac{\partial}{\partial t}\left(\mathcal{T}_{t}(k T)|\widetilde{0}(t, \epsilon)\rangle\right),
$$

which, using Eq. (10), may be written as

$$
H(t, \epsilon)|\widetilde{0}(t+k T, \epsilon)\rangle=i \frac{\partial}{\partial t}|\widetilde{0}(t+k T, \epsilon)\rangle .
$$

Comparing Eqs. (1) and (13) and assuming that $|\widetilde{0}(t, \epsilon)\rangle$ is nondegenerate, we conclude that $\widetilde{0}(t, \epsilon)\rangle$ and $|\widetilde{0}(t+k T, \epsilon)\rangle$ satisfy the same Schrödinger equation and they therefore differ at most by a constant phase factor,

$$
\widetilde{0}(t+k T, \epsilon)\rangle=\exp (-i k \alpha)|\widetilde{0}(t, \epsilon)\rangle
$$

where $\alpha$ is the phase that describes a time-translation over one period $T$. Since $\widetilde{0}(t, \epsilon)\rangle$ is unit-normalized at each time [Eq. (8)], $\alpha$ is real.

Eq. (14) is satisfied for a wave function of the form

$$
\widetilde{0}(t, \epsilon)\rangle=\exp \left(-i \frac{\alpha}{T} t\right)|u(t, \epsilon)\rangle,
$$

where $|u(t, \epsilon)\rangle$ is time-periodic with the same period $T$ as the Hamiltonian,

$$
|u(t+T, \epsilon)\rangle=|u(t, \epsilon)\rangle .
$$


This may be seen from the following derivation:

$$
\begin{aligned}
\widetilde{0}(t+k T, \epsilon)\rangle & =\exp \left[-i \frac{\alpha}{T}(t+k T)\right]|u(t+k T, \epsilon)\rangle \\
& =\exp (-i k \alpha) \exp \left(-i \frac{\alpha}{T} t\right)|u(t, \epsilon)\rangle \\
& =\exp (-i k \alpha)|\widetilde{0}(t, \epsilon)\rangle .
\end{aligned}
$$

From Eqs. (8) and (15), it follows that $|u(t, \epsilon)\rangle$ is unitnormalized,

$$
\langle u(t, \epsilon) \mid u(t, \epsilon)\rangle=1 .
$$

Introducing the notation

$$
\mathcal{E}=\frac{\alpha}{T}
$$

$\widetilde{0}(t, \epsilon)\rangle$ of Eq. (15) becomes

$$
\widetilde{0}(t, \epsilon)\rangle=\exp (-i \mathcal{E}(\epsilon) t)|u(t, \epsilon)\rangle .
$$

Inserting Eq. (20) into the Schrödinger equation [Eq. (1)] yields an eigenvalue equation from which $\mathcal{E}(\epsilon)$ and $|u(t, \epsilon)\rangle$ may be determined,

$$
\left(H(t, \epsilon)-i \frac{\partial}{\partial t}\right)|u(t, \epsilon)\rangle=\mathcal{E}(\epsilon)|u(t, \epsilon)\rangle .
$$

Note that $\mathcal{E}(\epsilon)$ depends on the set of perturbation strengths $\epsilon$. Equations (16) and (21) are the time-dependent Schrödinger equation within steady-state theory. ${ }^{6}$

\section{B. The regular wave function and its eigenvalue equation}

The steady-state wave function contains an undetermined time-dependent phase factor. To determine this phase factor, we introduce a reference state $\left|s_{0}\right\rangle$ which may be any normalized time-independent state that has a non-vanishing component in $|u(t, \epsilon)\rangle$,

$$
\left\langle s_{0} \mid u(t, \epsilon)\right\rangle \neq 0
$$

We then introduce the time-dependent regular wave function, $\left|0_{R}(t, \epsilon)\right\rangle$, defined as

$$
|u(t, \epsilon)\rangle=\frac{\left\langle s_{0} \mid u(t, \epsilon)\right\rangle}{\left|\left\langle s_{0} \mid u(t, \epsilon)\right\rangle\right|}\left|0_{R}(t, \epsilon)\right\rangle,
$$

where $\left|\left\langle s_{0} \mid u(t, \epsilon)\right\rangle\right|$ is the modulus of $\left\langle s_{0} \mid u(t, \epsilon)\right\rangle$. We now show that $\left|0_{R}(t, \epsilon)\right\rangle$ is connected to $|u(t, \epsilon)\rangle$ via a phase factor that has a real time-periodic phase, that $\left|0_{R}(t, \epsilon)\right\rangle$ is time-periodic and unit-normalized, and that it has a real positive component in the $\left|s_{0}\right\rangle$ direction.

As $\frac{\left\langle s_{0} \mid u(t, \epsilon)\right\rangle}{\left\langle s_{0} \mid u(t, \epsilon)\right\rangle \mid}$ has modulus 1 , it may be written in terms of a real phase $\mathcal{F}_{P_{0}}(t, \epsilon)$ as

$$
\frac{\left\langle s_{0} \mid u(t, \epsilon)\right\rangle}{\left|\left\langle s_{0} \mid u(t, \epsilon)\right\rangle\right|}=\exp \left(-i \mathcal{F}_{P_{0}}(t, \epsilon)\right) .
$$

Since $|u(t, \epsilon)\rangle$ is time-periodic, it follows from Eq. (24) that $\mathcal{F}_{P_{0}}(t, \epsilon)$ is also time-periodic. Substituting Eq. (24) in Eq. (23) gives as

$$
|u(t, \epsilon)\rangle=\exp \left(-i \mathcal{F}_{P_{0}}(t, \epsilon)\right)\left|0_{R}(t, \epsilon)\right\rangle
$$

From Eq. (25), we see that the time-periodicity of $|u(t, \epsilon)\rangle$ and $\mathcal{F}_{P_{0}}(t, \epsilon)$ implies that the regular wave function is time-periodic

$$
\left|0_{R}(t+T, \epsilon)\right\rangle=\left|0_{R}(t, \epsilon)\right\rangle .
$$

Using that the steady-state wave function is unit-normalized [Eq. (18)] and that $\mathcal{F}_{P_{0}}(t, \epsilon)$ is real, it follows from Eq. (25) that the regular wave function is unit-normalized,

$$
\left\langle 0_{R}(t, \epsilon) \mid 0_{R}(t, \epsilon)\right\rangle=1 .
$$

Taking the scalar product of $|u(t, \epsilon)\rangle$ in Eq. (23) with $\left\langle s_{0}\right|$ gives

$$
\left\langle s_{0} \mid 0_{R}(t, \epsilon)\right\rangle=\left|\left\langle s_{0} \mid u(t, \epsilon)\right\rangle\right|>0 .
$$

Inserting Eq. (25) in Eq. (20) shows the connection between the full solution to the Schrödinger equation and the regular wave function,

$$
\widetilde{0}(t, \epsilon)\rangle=\exp (-i \mathcal{F}(t, \epsilon))\left|0_{R}(t, \epsilon)\right\rangle,
$$

where

$$
\mathcal{F}(t, \epsilon)=\mathcal{E}(\epsilon) t+\mathcal{F}_{P_{0}}(t, \epsilon) .
$$

Substituting Eq. (25) in the Schrödinger equation for the steady-state wave function [Eq. (21)] gives

$$
\begin{gathered}
\left(H(t, \epsilon)-i \frac{\partial}{\partial t}\right) \exp \left(-i \mathcal{F}_{P_{0}}(t, \epsilon)\right)\left|0_{R}(t, \epsilon)\right\rangle \\
=\mathcal{E}(\epsilon) \exp \left(-i \mathcal{F}_{P_{0}}(t, \epsilon)\right)\left|0_{R}(t, \epsilon)\right\rangle,
\end{gathered}
$$

which may be expressed as

$$
\left(H(t, \epsilon)-i \frac{\partial}{\partial t}-\dot{\mathcal{F}}_{P_{0}}(t, \epsilon)\right)\left|0_{R}(t, \epsilon)\right\rangle=\mathcal{E}(\epsilon)\left|0_{R}(t, \epsilon)\right\rangle .
$$

Eq. (32) is an eigenvalue equation with the eigenvalue $\mathcal{E}(\epsilon)$ and the eigenstate $\left|0_{R}(t, \epsilon)\right\rangle$. The regular wave function thus is a phase-isolated solution to the time-dependent Schrödinger equation.

The phase [Eq. (30)] is not uniquely defined as $\mathcal{F}_{P_{0}}(t, \epsilon)$ [Eq. (24)] depends on the choice of the reference state $\left|s_{0}\right\rangle$. In Sec. II C, we introduce a composite Hilbert space ${ }^{6}$ and show that in this space both Eqs. (21) and (32) are Hermitian eigenvalue equations and that the solution to Eq. (32) becomes independent of $\mathcal{F}_{P_{0}}(t, \epsilon)$.

\section{The Hermiticity of the time-dependent Hamiltonian}

We now introduce a composite Hilbert space in the time and configuration space variables for solving Eqs. (21) and (32). First, we recognize that the functions $\exp \left(i p \omega_{T} t\right)$, with $\omega_{T}$ defined in Eq. (6) and $p=0, \pm 1, \pm 2, \ldots$, satisfy

$$
\frac{1}{T} \int_{0}^{T}\left[\exp \left(i p \omega_{T} t\right)\right]^{*} \exp \left(i q \omega_{T} t\right) \mathrm{d} t=\delta_{p q}
$$

and form a complete orthonormal basis for the Hilbert space in the time variable, where the inner product for two timeperiodic functions $f(t)$ and $g(t)$ is defined as

$$
\left\{f^{*}(t) g(t)\right\}_{T}=\frac{1}{T} \int_{0}^{T} f^{*}(t) g(t) \mathrm{d} t .
$$


Since $|u(t, \epsilon)\rangle$ is time-periodic with the period $T$, we may expand $|u(t, \epsilon)\rangle$ as

$$
|u(t, \epsilon)\rangle=\sum_{m p} c_{m p} \exp \left(i p \omega_{T} t\right)|m\rangle,
$$

where $\{|m\rangle\}$ is an orthonormal basis in the Hilbert space over configuration space variables and the coefficients $c_{m p}$ are timeindependent. The eigenstate $|u(t, \epsilon)\rangle$ of Eq. (35) therefore may be expanded in the composite Hilbert space comprehending $^{6}$ both the time and the configuration space variables.

The inner product in the composite Hilbert space is

$$
\{\langle a(t) \mid b(t)\rangle\}_{T}=\frac{1}{T} \int_{0}^{T}\langle a(t) \mid b(t)\rangle \mathrm{d} t,
$$

where $\langle a(t) \mid b(t)\rangle$ denotes the standard inner product over the configuration space variables. The time average of a time derivative of a time-periodic function vanishes

$$
\begin{aligned}
f(t+T) & =f(t) \Rightarrow\left\{\frac{\mathrm{d} f(t)}{\mathrm{d} t}\right\}_{T}=\frac{1}{T} \int_{0}^{T} \frac{\mathrm{d} f(t)}{\mathrm{d} t} \mathrm{~d} t \\
& =\frac{1}{T}[f(t)]_{0}^{T}=\frac{1}{T}(f(T)-f(0))=0 .
\end{aligned}
$$

The operator $i \frac{\partial}{\partial t}$ is Hermitian in the composite Hilbert space, since for two time-periodic states $\langle a(t)|$ and $|b(t)\rangle$ with the period $T$, we have from Eq. (37)

$$
\begin{aligned}
0 & =\left\{i \frac{\partial}{\partial t}\langle a(t) \mid b(t)\rangle\right\}_{T} \\
& =-\left\{\left\langle i \frac{\partial}{\partial t} a(t) \mid b(t)\right\rangle\right\}_{T}+\left\{\left\langle a(t) \mid i \frac{\partial}{\partial t} b(t)\right\rangle\right\}_{T}
\end{aligned}
$$

and thus

$$
\left\{\left\langle i \frac{\partial}{\partial t} a(t) \mid b(t)\right\rangle\right\}_{T}=\left\{\left\langle a(t) \mid i \frac{\partial}{\partial t} b(t)\right\rangle\right\}_{T} .
$$

Since the Hamiltonian $H(t, \epsilon)$ of Eq. (2) is Hermitian, it follows from Eq. (39) that the operator $\left(H-i \frac{\partial}{\partial t}\right)$ is Hermitian in the composite Hilbert space and Eqs. (21) and (32) constitute standard Hermitian eigenvalue equations in this space. ${ }^{6}$ The regular wave-function is also periodic and may therefore also be expressed in the composite Hilbert space.

The time average of the time-differentiated overall phasefactor, Eq. (30), gives

$$
\{\dot{\mathcal{F}}(t, \epsilon)\}_{T}=\mathcal{E}(\epsilon)+\left\{\dot{\mathcal{F}}_{P_{0}}(t, \epsilon)\right\}_{T}
$$

Using that $\mathcal{F}_{P_{0}}(t, \epsilon)$ is periodic, Eq. (37) may be invoked

$$
\left\{\dot{\mathcal{F}}_{P_{0}}(t, \epsilon)\right\}_{T}=0
$$

and Eq. (40) becomes

$$
\{\dot{\mathcal{F}}(t, \epsilon)\}_{T}=\mathcal{E}(\epsilon) .
$$

Whereas the phase $\mathcal{F}(t, \epsilon)$ is not uniquely defined and depends on the choice of the state $\left|s_{0}\right\rangle$, the time average of the time derivative of the phase, $\{\dot{\mathcal{F}}(t, \epsilon)\}_{T}$, is independent of that choice of $\left|s_{0}\right\rangle$ and uniquely defined ${ }^{76}$ and equal to $\mathcal{E}(\epsilon)$.

\section{The Hermitian eigenvalue equation in the limit of a time-independent perturbation}

In the limit, where the frequencies in Eq. (3) are zero, the perturbation operator of Eq. (3) and hence also the Hamiltonian of Eq. (2) become time-independent

$$
H(\epsilon)=H_{0}+V(\epsilon)
$$

and the time-dependent Schrödinger equation reads

$$
H(\epsilon)\left|\widetilde{0}_{0}(t, \epsilon)\right\rangle=i \frac{\partial}{\partial t}\left|\widetilde{0}_{0}(t, \epsilon)\right\rangle .
$$

The solution to the time-dependent Schrödinger equation [Eq. (44)] may then be parametrized as

$$
\left|\widetilde{0}_{0}(t, \epsilon)\right\rangle=\exp \left(-i E_{0}(\epsilon) t\right)\left|\widetilde{0}_{0}(\epsilon)\right\rangle,
$$

which, inserted in Eq. (44), gives the time-independent Schrödinger equation

$$
H(\epsilon)\left|\widetilde{0}_{0}(\epsilon)\right\rangle=E_{0}(\epsilon)\left|\widetilde{0}_{0}(\epsilon)\right\rangle .
$$

The eigenstate $\left|\widetilde{0}_{0}(\epsilon)\right\rangle$ contains an undetermined timeindependent phase factor,

$$
\left.\widetilde{0}_{0}(\epsilon)\right\rangle=\exp (-i \phi(\epsilon))\left|0_{0}(\epsilon)\right\rangle .
$$

Inserting Eq. (47) in Eq. (46) gives

$H(\epsilon) \exp (-i \phi(\epsilon))\left|0_{0}(\epsilon)\right\rangle=E_{0}(\epsilon) \exp (-i \phi(\epsilon))\left|0_{0}(\epsilon)\right\rangle$.

The phase factor is constant and may therefore straightforwardly be eliminated giving the time-independent Schrödinger equation for the phase-isolated state,

$$
H(\epsilon)\left|0_{0}(\epsilon)\right\rangle=E_{0}(\epsilon)\left|0_{0}(\epsilon)\right\rangle .
$$

We now consider the time-independent limit of the timedependent Schrödinger equation [Eq. (1)]. In this limit, the steady-state eigenvalue equation [Eq. (21)] becomes the timeindependent Schrödinger equation for the phase-including state [Eq. (46)]. Comparing Eqs. (25) and (47), we see that in the time-independent limit the phase factor $\exp \left(-i \mathcal{F}_{P_{0}}(t, \epsilon)\right)$ becomes time-independent and equal to $\exp (-i \phi(\epsilon))$, whereas the regular wave function $\left|0_{R}(t, \epsilon)\right\rangle$ becomes equal to $\left|0_{0}(\epsilon)\right\rangle$. For the time-independent Schrödinger equation [Eq. (48)], the time-independent phase factor could be removed giving the time-independent Schrödinger equation [Eq. (49)]. For the time-dependent Schrödinger eigenvalue equation [Eq. (31)], the time-dependent phase factor introduces the time derivative of the phase, $\dot{\mathcal{F}}_{P_{0}}(t, \epsilon)$, in the eigenvalue equation [Eq. (32)], which of course vanishes in the time-independent limit.

Summarizing, in the time-independent limit, we have

$$
\begin{gathered}
\mathcal{E}(\epsilon) \longrightarrow E_{0}(\epsilon), \\
\left.|u(t, \epsilon)\rangle \longrightarrow \widetilde{0}_{0}(\epsilon)\right\rangle, \\
\left|0_{R}(t, \epsilon)\right\rangle \longrightarrow\left|0_{0}(\epsilon)\right\rangle,
\end{gathered}
$$

and

$$
\mathcal{F}_{P_{0}}(t, \epsilon) \longrightarrow \phi(\epsilon) .
$$

The eigenvalue $\mathcal{E}(\epsilon)$ is thus a generalization of the energy to time-periodic perturbations. $\mathcal{E}(\epsilon)$ is therefore referred to as the quasi-energy. ${ }^{6}$ 


\section{E. The variational principle for the quasi-energy}

The eigenvalue $\mathcal{E}(\epsilon)$ may be determined by projecting, in the composite Hilbert space, either the Hermitian eigenvalue equation [Eq. (21)] against $\langle u(t, \epsilon)|$,

$$
\mathcal{E}(\epsilon)=\left\{\left\langle u(t, \epsilon)\left|\left(H(t, \epsilon)-i \frac{\partial}{\partial t}\right)\right| u(t, \epsilon)\right\rangle\right\}_{T},
$$

or the Hermitian eigenvalue equation [Eq. (32)] against $\left\langle 0_{R}(t, \epsilon)\right|$,

$$
\mathcal{E}(\epsilon)=\left\{\left\langle 0_{R}(t, \epsilon)\left|\left(H(t, \epsilon)-i \frac{\partial}{\partial t}\right)\right| 0_{R}(t, \epsilon)\right\rangle\right\}_{T},
$$

where we have used the unit normalization of $|u(t, \epsilon)\rangle$ [Eq. (18)] and $\left|0_{R}(t, \epsilon)\right\rangle$ [Eq. (27)]. To obtain Eq. (55), we have also used Eq. (41). In the following, we will determine $\mathcal{E}(\epsilon)$ expressed in terms of $\left|0_{R}(t, \epsilon)\right\rangle$ [Eq. (55)], rather than in terms of $|u(t, \epsilon)\rangle$ [Eq. (54)], since $\left|0_{R}(t, \epsilon)\right\rangle$, and not $|u(t, \epsilon)\rangle$, becomes equal to $\left|0_{0}(\epsilon)\right\rangle$ in the limit of static perturbation.

$\mathcal{E}(\epsilon)$ of Eq. (55) is a generalization of the expectation value expression for the energy for a time-independent system

$$
E_{0}(\epsilon)=\left\langle 0_{0}(\epsilon)|H(\epsilon)| 0_{0}(\epsilon)\right\rangle .
$$

$\mathcal{E}(\epsilon)$ is also an eigenvalue of the Hermitian eigenvalue equation [Eq. (32)] which is a generalization of the eigenvalue of the Hermitian eigenvalue equation for a time-independent Hamiltonian [Eq. (49)]. For the time-independent perturbed system, the variational principle for the ground-state energy of Eq. (56) becomes

$$
\delta E_{0}(\epsilon)=\left\langle\delta 0_{0}(\epsilon)|H(\epsilon)| 0_{0}(\epsilon)\right\rangle+\left\langle 0_{0}(\epsilon)|H(\epsilon)| \delta 0_{0}(\epsilon)\right\rangle=0
$$

and is equivalent to solving the time-independent Schrödinger equation [Eq. (49)]. Similarly, for a time-periodic system, the variational principle for $\mathcal{E}(\epsilon)$ in Eq. (55)

$$
\begin{aligned}
\delta \mathcal{E}(\epsilon)= & \left\{\left\langle\delta 0_{R}(t, \epsilon)\left|\left(H(t, \epsilon)-i \frac{\partial}{\partial t}\right)\right| 0_{R}(t, \epsilon)\right\rangle\right\}_{T} \\
& +\left\{\left\langle 0_{R}(t, \epsilon)\left|\left(H(t, \epsilon)-i \frac{\partial}{\partial t}\right)\right| \delta 0_{R}(t, \epsilon)\right\rangle\right\}_{T}=0
\end{aligned}
$$

is equivalent to solving the Hermitian eigenvalue equation [Eq. (32)]. This equivalence between a variational principle and a Hermitian eigenvalue equation is a general equivalence and is discussed for time-independent theory, for example, in Ref. 82. Alternatively, the equivalence may be shown treating $\mathcal{E}(\epsilon)$ of Eq. (55) as the action functional in the same manner as in Refs. 83-85.

When the variational principle is written in the form of Eq. (58), we have used that the time-periodic regular wave function $\left|0_{R}(t, \epsilon)\right\rangle$ is unit-normalized for all perturbation strengths and at each time [Eq. (27)]. The allowed variations in Eq. (58) are therefore time-periodic variations which according to Eq. (27) fulfill the normalization condition

$$
\left\langle 0_{R}(t, \epsilon)+\delta 0_{R}(t, \epsilon) \mid 0_{R}(t, \epsilon)+\delta 0_{R}(t, \epsilon)\right\rangle=1 .
$$

This implies that through first-order, the variations satisfy

$$
\left\langle\delta 0_{R}(t, \epsilon) \mid 0_{R}(t, \epsilon)\right\rangle+\left\langle 0_{R}(t, \epsilon) \mid \delta 0_{R}(t, \epsilon)\right\rangle=0
$$

corresponding to

$$
\left\langle 0_{R}(t, \epsilon) \mid \delta 0_{R}(t, \epsilon)\right\rangle=-\left\langle 0_{R}(t, \epsilon) \mid \delta 0_{R}(t, \epsilon)\right\rangle^{*} .
$$

Equation (61) shows that for an allowed variation satisfying Eq. (58), $\left\langle 0_{R}(t, \epsilon) \mid \delta 0_{R}(t, \epsilon)\right\rangle$ must either vanish or be purely imaginary. Similar allowed variations may be applied to obtain Eq. (57).

\section{F. Molecular response properties as derivatives of the eigenvalue of the Hermitian eigenvalue equation}

We will now discuss how molecular response properties may be determined from the quasi-energy $\mathcal{E}(\epsilon)$. Consider the limit, where the frequencies of the perturbation of Eq. (3) are zero. The quasi-energy $\mathcal{E}(\epsilon)$ then becomes the ground-state energy, $E_{0}(\epsilon)$, of the perturbed time-independent system. For the perturbations described by the Hamiltonian of Eq. (43), the induced changes of the ground state energy may be experimentally observed for general values of the perturbation strengths. It is therefore experimentally possible to determine an order expansion of the ground state energy, which directly gives the static molecular properties. Carrying out a Taylor series expansion of $E_{0}(\epsilon)$ in the perturbation strengths around the ground-state energy $E_{0}$ of the unperturbed system

$$
E_{0}(\epsilon)=E_{0}+\sum_{j_{1}} E_{0}^{X_{j_{1}}} \epsilon_{X_{j_{1}}}+\frac{1}{2} \sum_{j_{1} j_{2}} E_{0}^{X_{j_{1}} X_{j_{2}}} \epsilon_{X_{j_{1}}} \epsilon_{X_{j_{2}}}+\cdots,
$$

we may thus identify static molecular properties for the state $\left|0_{0}\right\rangle$ of the energy $E_{0}$ as the expansion coefficients of the Taylor series

$$
E_{0}^{X_{j_{1}} \cdots X_{j_{n}}}=\left(\frac{\partial^{n} E_{0}(\epsilon)}{\partial \epsilon_{X_{j_{1}}} \ldots \partial \epsilon_{X_{j_{n}}}}\right),
$$

where the index 0 denotes that the derivative is taken at zero perturbation strength.

For a time-periodic Hamiltonian [Eq. (2)], the quasienergy of Eq. (55) may also be determined in each order of the perturbation strengths identifying molecular constants denoted the frequency-dependent molecular properties or the molecular response functions. Carrying out a Taylor series expansion of $\mathcal{E}(\epsilon)$ in the perturbation strengths around the ground-state energy $E_{0}$ of the unperturbed system

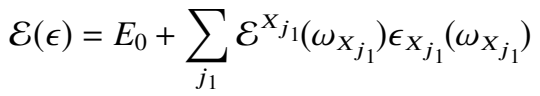

$$
\begin{aligned}
& +\frac{1}{2} \sum_{j_{1} j_{2}} \mathcal{E}^{X_{j_{1}} X_{j_{2}}}\left(\omega_{X_{j 1}}, \omega_{X_{j 2}}\right) \\
& \times \epsilon_{X_{j 1}}\left(\omega_{X_{j 1}}\right) \epsilon_{X_{j 2}}\left(\omega_{X_{j 2}}\right)+\cdots,
\end{aligned}
$$

we may thus identify the frequency-dependent molecular properties for the state $\left|0_{0}\right\rangle$ of the energy $E_{0}$ as the expansion coefficients of the Taylor series

$$
\begin{aligned}
\mathcal{E}^{X_{j_{1}} \ldots X_{j_{n}}}\left(\omega_{X_{j_{1}}}, \ldots, \omega_{X_{j_{n}}}\right) \\
=\left(\frac{\partial^{n} \mathcal{E}(\epsilon)}{\partial \epsilon_{X_{j_{1}}}\left(\omega_{X_{j_{1}}}\right) \ldots \partial \epsilon_{X_{j_{n}}}\left(\omega_{X_{j_{n}}}\right)}\right),
\end{aligned}
$$

where $\mathcal{E}^{X_{j_{1}} \ldots X_{j_{n}}}\left(\omega_{X_{j_{1}}}, \ldots, \omega_{X_{j_{n}}}\right)$ is symmetric with respect to permutations of operator-frequency pairs

$$
\left(X_{j_{k}}, \omega_{X_{j_{k}}}\right) \leftrightarrow\left(X_{j_{l}}, \omega_{X_{j_{l}}}\right), \quad k, l=1,2, \ldots n .
$$


Eq. (65) thus is a generalization of Eq. (63) to timeperiodic perturbations and determines the molecular response functions.

The Hamiltonian $H_{0}$ for the unperturbed system satisfies the eigenvalue equation

$$
H_{0}\left|0_{m}\right\rangle=E_{m}\left|0_{m}\right\rangle .
$$

Since the eigenstates of $H_{0}$ form a complete set, the regular wave function, $\left|0_{R}(t, \epsilon)\right\rangle$ in Eq. (32), may be expressed as

$$
\left|0_{R}(t, \epsilon)\right\rangle=N(t, \epsilon)\left(\left|0_{0}\right\rangle+\sum_{m \neq 0} b_{m}(t, \epsilon)\left|0_{m}\right\rangle\right),
$$

where the reference state $\left|s_{0}\right\rangle$ that has a real and positive expansion coefficient in $\left|0_{R}(t, \epsilon)\right\rangle$ [Eq. (28)] has been chosen as $\left|0_{0}\right\rangle$.

The regular wave function $\left|0_{R}(t, \epsilon)\right\rangle$ describes the time evolution of the perturbed ground state and thus determines the molecular response properties for this state. However, through the time-evolving coefficients $b_{m}(t, \epsilon)$ [Eq. (68)], the regular wave function also describes how transitions may be induced between the ground and excited states and between excited states and further probes the molecular properties of the excited states. Consider, for example, the second-order quasi-

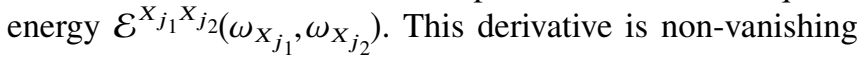
only for $\omega_{X_{j_{1}}}+\omega_{X_{j_{2}}}=0$ [see Sec. III B 1, in particular Eq. (142)] and may be expanded in the basis of eigenfunctions $\left|0_{m}\right\rangle$ as

$$
\begin{aligned}
\mathcal{E}^{X_{j_{1}} X_{j_{2}}\left(\omega_{X_{j_{1}}}, \omega_{X_{j_{2}}}\right)=} \sum_{m}\left(\frac{\left\langle 0_{0}\left|X_{j_{1}}\right| 0_{m}\right\rangle\left\langle 0_{m}\left|X_{j_{2}}\right| 0_{0}\right\rangle}{\omega_{X_{j_{2}}}-\left(E_{m}-E_{0}\right)}\right. \\
\left.+\frac{\left\langle 0_{0}\left|X_{j_{2}}\right| 0_{m}\right\rangle\left\langle 0_{m}\left|X_{j_{1}}\right| 0_{0}\right\rangle}{-\omega_{X_{j_{2}}}-\left(E_{m}-E_{0}\right)}\right) .
\end{aligned}
$$

Equation (69) shows that the second-order quasi-energy becomes infinite whenever an experimental frequency, $\omega_{X_{j_{2}}}$, matches an excitation energy, $E_{m 0}=E_{m}-E_{0}$. Similarly, the general-order quasi-energy, $\mathcal{E}^{X_{j_{1}} \ldots X_{j_{n}}}\left(\omega_{X_{j_{1}}}, \ldots, \omega_{X_{j_{n}}}\right)$ in Eq. (65), becomes infinite whenever one of the frequencies matches an excitation energy. For this match, molecular properties are determined by the residues of the second- and higher-order energy corrections, as the energy corrections only contain simple poles. For example, when the experimental frequency $\omega_{X_{k}}$ matches $E_{m 0}=E_{m}-E_{0}$, the simple pole for $\omega_{X_{k}}=E_{m 0}$ can be removed by taking the limit

$$
\begin{aligned}
& \lim _{\omega_{X_{k}} \rightarrow E_{m 0}}\left(\omega_{X_{k}}-E_{m 0}\right) \mathcal{E}(\epsilon) \\
& =\frac{1}{2} \lim _{\omega_{X_{k}} \rightarrow E_{m 0}}\left(\omega_{X_{k}}-E_{m 0}\right) \\
& \times \sum_{j_{1} j_{2}} \mathcal{E}^{X_{j_{1}} X_{j_{2}}} \epsilon_{X_{j_{1}}}\left(\omega_{X_{j_{1}}}\right) \epsilon_{X_{j_{2}}}\left(\omega_{X_{j_{2}}}\right) \\
& +\frac{1}{6} \lim _{\omega_{X_{k}} \rightarrow E_{m 0}}\left(\omega_{X_{k}}-E_{m 0}\right) \sum_{j_{1} j_{2} j_{3}} \mathcal{E}^{X_{j_{1}} X_{j_{2}} X_{j_{3}}} \\
& \times \epsilon_{X_{j_{1}}}\left(\omega_{X_{j_{1}}}\right) \epsilon_{X_{j_{2}}}\left(\omega_{X_{j_{2}}}\right) \epsilon_{X_{j_{3}}}\left(\omega_{X_{j_{3}}}\right)+\cdots
\end{aligned}
$$

and residues are determined at each order in the perturbation. These residues are molecular constants and determine the molecular properties that describe the strength of the transitions that are induced by the perturbation of the frequency $\omega_{X_{k}}$ between the ground state $\left|0_{0}\right\rangle$ and the excited state $\left|0_{m}\right\rangle$. For example, for the second-order quasi-energy in Eq. (69), we obtain

$$
\begin{aligned}
\lim _{\omega_{X_{2}} \rightarrow \omega}\left(\omega_{X_{2}}-\omega\right) \mathcal{E}^{X_{j_{1}} X_{j_{2}}\left(\omega_{X_{j_{1}}}, \omega_{X_{j_{2}}}\right)} & \\
= & \sum_{m: E_{m}-E_{0}=\omega}\left\langle 0_{0}\left|X_{j_{1}}\right| 0_{m}\right\rangle\left\langle 0_{m}\left|X_{j_{2}}\right| 0_{0}\right\rangle,
\end{aligned}
$$

where the summation is restricted to excited states with energies fulfilling $E_{m}-E_{0}=\omega$. If $\omega$ matches an excitation energy $E_{m}-E_{0}$ and the corresponding excited state is nondegenerate, we obtain

$$
\begin{aligned}
& \lim _{\omega_{X_{2}} \rightarrow\left(E_{m}-E_{0}\right)}\left(\omega_{X_{2}}-\left(E_{m}-E_{0}\right)\right) \mathcal{E}^{X_{j_{1}} X_{j_{2}}\left(\omega_{X_{j_{1}}}, \omega_{X_{j_{2}}}\right)} \\
& =\left\langle 0_{0}\left|X_{j_{1}}\right| 0_{m}\right\rangle\left\langle 0_{m}\left|X_{j_{2}}\right| 0_{0}\right\rangle
\end{aligned}
$$

showing that the ground to excited state transition strengths may be obtained from the residues of the frequency-dependent second-order quasi-energy.

Similarly, we have double residues as $\lim _{\omega_{X_{k}} \rightarrow E_{m 0}}\left(\omega_{X_{k}}-\right.$ $\left.E_{m 0}\right)\left(\lim _{\omega_{X_{l}} \rightarrow E_{n 0}}\left(\omega_{X_{l}}-E_{n 0}\right) \mathcal{E}(\epsilon)\right)$, which determine transition properties between two excited states $\left|0_{m}\right\rangle$ and $\left|0_{n}\right\rangle$. In particular, if $\left|0_{m}\right\rangle=\left|0_{n}\right\rangle$, this residue determines molecular properties for the excited state $\left|0_{m}\right\rangle$. Transition properties and molecular properties of the excited states are inaccessible in time-independent theory, as static perturbations cannot be targeted to excite to a single excited state.

In this article, we focus on determining the ground-state response functions as derivatives of the quasi-energy (see Eq. (65)) and refer to Refs. 1, 2, and 76 for a detailed discussion how the transition properties and molecular properties of excited states may be determined from the residues of the ground-state response functions.

\section{G. Size-extensivity of the quasi-energy and molecular properties}

In this subsection, we examine, for time-periodic perturbations, the size-extensivity of the exact wave-function and the quasi-energy. Consider two molecular systems $C, D$ that are located infinitely apart. The two systems do not interact, so the Hamiltonian for the combined system may be written

$$
H_{C D}(t, \epsilon)=H_{C}(t, \epsilon)+H_{D}(t, \epsilon)
$$

where $H_{C}(t, \epsilon)$ and $H_{D}(t, \epsilon)$ are the time-dependent Hamiltonians for system $C$ and $D$, respectively. The steady-state wave functions for the two sub-systems, $\left|u_{C}(t, \epsilon)\right\rangle$ and $\left|u_{D}(t, \epsilon)\right\rangle$, satisfy

$$
\begin{aligned}
& \left(H_{C}(t, \epsilon)-i \frac{\partial}{\partial t}\right)\left|u_{C}(t, \epsilon)\right\rangle=\mathcal{E}_{C}(\epsilon)\left|u_{C}(t, \epsilon)\right\rangle, \\
& \left(H_{D}(t, \epsilon)-i \frac{\partial}{\partial t}\right)\left|u_{D}(t, \epsilon)\right\rangle=\mathcal{E}_{D}(\epsilon)\left|u_{D}(t, \epsilon)\right\rangle .
\end{aligned}
$$


From Eq. (74), it follows that the direct product wave function $\left|u_{C}(t, \epsilon) u_{D}(t, \epsilon)\right\rangle$ satisfies

$$
\begin{array}{r}
\left(H_{C}(t, \epsilon)+H_{D}(t, \epsilon)-i \frac{\partial}{\partial t}\right)\left|u_{C}(t, \epsilon) u_{D}(t, \epsilon)\right\rangle \\
=\left|\left[\left(H_{C}(t, \epsilon)-i \frac{\partial}{\partial t}\right) u_{C}(t, \epsilon)\right] u_{D}(t, \epsilon)\right\rangle \\
+\left|u_{C}(t, \epsilon)\left(H_{D}(t, \epsilon)-i \frac{\partial}{\partial t}\right) u_{D}(t, \epsilon)\right\rangle \\
=\left(\mathcal{E}_{C}(\epsilon)+\mathcal{E}_{D}(\epsilon)\right)\left|u_{C}(t, \epsilon) u_{D}(t, \epsilon)\right\rangle,
\end{array}
$$

showing that for a multiplicatively separable steady-state wave function

$$
\left|u_{C D}(t, \epsilon)\right\rangle=\left|u_{C}(t, \epsilon) u_{D}(t, \epsilon)\right\rangle,
$$

the quasi-energy is additively separable

$$
\mathcal{E}_{C D}(\epsilon)=\mathcal{E}_{C}(\epsilon)+\mathcal{E}_{D}(\epsilon) .
$$

We now show that the regular wave function is also multiplicatively separable. To see this, we consider the phase factor that connects the regular and steady-state wave function in Eq. (23),

$$
\frac{\left\langle s_{0, C D} \mid u_{C D}(t, \epsilon)\right\rangle}{\left|\left\langle s_{0, C D} \mid u_{C D}(t, \epsilon)\right\rangle\right|}=\frac{\left\langle s_{0, C} \mid u_{C}(t, \epsilon)\right\rangle}{\left|\left\langle s_{0, C} \mid u_{C}(t, \epsilon)\right\rangle\right|} \frac{\left\langle s_{0, D} \mid u_{D}(t, \epsilon)\right\rangle}{\left|\left\langle s_{0, D} \mid u_{D}(t, \epsilon)\right\rangle\right|},
$$

where the reference wave function for the compound system, $\left|s_{0, C D}\right\rangle=\left|s_{0, C} s_{0, D}\right\rangle$, is assumed to be multiplicatively separable. Eq. (78) implies that the phase $\mathcal{F}_{P_{0}}(t, \epsilon)$ of Eq. (24) is additively separable,

$$
\mathcal{F}_{P_{0, C D}}(t, \epsilon)=\mathcal{F}_{P_{0, C}}(t, \epsilon)+\mathcal{F}_{P_{0, D}}(t, \epsilon),
$$

and the connection between the steady-state and the regular wave functions may therefore for two non-interacting subsystems $C$ and $D$ be written as

$$
\begin{aligned}
\left|u_{C}(t, \epsilon) u_{D}(t, \epsilon)\right\rangle= & \exp \left(-i \mathcal{F}_{P_{0, C}}(t, \epsilon)\right) \\
& \times \exp \left(-i \mathcal{F}_{P_{0, D}}(t, \epsilon)\right)\left|0_{R, C D}(t, \epsilon)\right\rangle .
\end{aligned}
$$

It follows from Eq. (80) that the regular wave function is multiplicatively separable,

$$
\left|0_{R, C D}(t, \epsilon)\right\rangle=\left|0_{R, C}(t, \epsilon) 0_{R, D}(t, \epsilon)\right\rangle .
$$

Equations (77) and (79) further show that the overall phase [Eq. (30)] is additively separable,

$$
\mathcal{F}_{C D}(t, \epsilon)=\mathcal{F}_{P_{0, C D}}(t, \epsilon)+\mathcal{E}_{C D}(\epsilon)=\mathcal{F}_{C}(t, \epsilon)+\mathcal{F}_{D}(t, \epsilon) .
$$

The full solution to the Schrödinger equation [Eq. (29)] is multiplicatively separable and Eqs. (81) and (82) show that it may be written in the form

$$
\begin{aligned}
\left|\widetilde{0}_{C D}(t, \epsilon)\right\rangle= & \exp \left(-i \mathcal{F}_{C D}(t, \epsilon)\right)\left|0_{R, C D}(t, \epsilon)\right\rangle \\
= & \exp \left(-i \mathcal{F}_{C}(t, \epsilon)\right) \exp \left(-i \mathcal{F}_{D}(t, \epsilon)\right) \\
& \times\left|0_{R, C}(t, \epsilon) 0_{R, D}(t, \epsilon)\right\rangle=\left|\widetilde{0}_{C}(t, \epsilon) \widetilde{0}_{D}(t, \epsilon)\right\rangle .
\end{aligned}
$$

Molecular properties were in Eq. (65) identified as derivatives of the quasi-energy with respect to the pertur- bation strengths. As the quasi-energy is additively separable [Eq. (77)] for all perturbation strengths, the molecular properties are also additively separable

$$
\begin{aligned}
\mathcal{E}_{C D}^{X_{j_{1}} \ldots X_{j_{n}}}\left(\omega_{X_{j_{1}}}, \ldots, \omega_{X_{j_{n}}}\right)= & \left(\frac{\partial^{n} \mathcal{E}_{C D}(\epsilon)}{\partial \epsilon_{X_{j_{1}}}\left(\omega_{X_{j_{1}}}\right) \ldots \partial \epsilon_{X_{j_{n}}}\left(\omega_{X_{j_{n}}}\right)}\right)_{0} \\
= & \mathcal{E}_{C}^{X_{j_{1}} \ldots X_{j_{n}}}\left(\omega_{X_{j_{1}}}, \ldots, \omega_{X_{j_{n}}}\right) \\
& +\mathcal{E}_{D}^{X_{j_{1}} \ldots X_{j_{n}}}\left(\omega_{X_{j_{1}}}, \ldots, \omega_{X_{j_{n}}}\right) .
\end{aligned}
$$

The frequency-dependent energy-corrections become infinite when a frequency matches an excitation energy of the system [see Eq. (69)]. The excitation energies are properties of the individual molecules and are thus an intensive property. However, as the energy-corrections are additively separable [Eq. (84)], so are the corresponding residues. For example, for the residue of second-order energy correction, we obtain from Eq. (71)

$$
\begin{aligned}
\lim _{X_{2} \rightarrow \omega} & \left(\omega_{X_{2}}-\omega\right) \mathcal{E}_{C D}^{X_{j_{1}} X_{j_{2}}}\left(\omega_{X_{j_{1}}}, \omega_{X_{j_{2}}}\right) \\
= & \sum_{m: E_{m, C^{-}-E_{0, C}=\omega}}\left\langle 0_{0, C}\left|X_{j_{1}}\right| 0_{m, C}\right\rangle\left\langle 0_{m, C}\left|X_{j_{2}}\right| 0_{0, C}\right\rangle \\
& +\sum_{m: E_{m, D^{-}} E_{0, D}=\omega}\left\langle 0_{0, D}\left|X_{j_{1}}\right| 0_{m, D}\right\rangle\left\langle 0_{m, D}\left|X_{j_{2}}\right| 0_{0, D}\right\rangle .
\end{aligned}
$$

If the systems $C$ and $D$ are identical and the excited state $m$ is non-degenerate for the individual systems, we obtain from Eq. (85)

$$
\begin{aligned}
& \lim _{\omega_{X_{2} \rightarrow \omega} \rightarrow}\left(\omega_{X_{2}}-\omega\right) \mathcal{E}_{C C}^{X_{j_{1}} X_{j_{2}}}\left(\omega_{X_{j_{1}}}, \omega_{X_{j_{2}}}\right) \\
& \quad=2\left\langle 0_{0, C}\left|X_{j_{1}}\right| 0_{m, C}\right\rangle\left\langle 0_{m, C}\left|X_{j_{2}}\right| 0_{0, C}\right\rangle,
\end{aligned}
$$

showing the size-extensivity of the transition strengths.

In the above, size-extensivity is defined as additive separability of, for example, the energy for a set of noninteracting systems. In the nomenclature of Bartlett, ${ }^{86}$ this property is referred to as size-consistency, whereas sizeextensivity refers to the scaling of a set of weakly interacting systems. For a thorough discussion of the relation between these two concepts, we refer to Ref. 87. In the present context, only size-extensivity in our restricted meaning of the word can be proven using the above general arguments. For the scaling of weakly interacting systems, an analysis of a specific method is required and we will return to this when discussing specific methods.

In Sec. III, we show in detail how response functions may be determined as derivatives of the quasi-energy, when the Hermitian eigenvalue equation [Eq. (32)] is solved by applying the variational principle of Eq. (58). We also show how the explicit time-dependence may be averaged out in the composite Hilbert space and replaced by a frequency dependence. In Sec. IV, we carry out a similar development, where the Hermitian eigenvalue equation is solved via projection. 


\section{RESPONSE FUNCTIONS FROM THE HERMITIAN EIGENVALUE EQUATION SOLVED USING THE VARIATIONAL PRINCIPLE}

In this section, we determine molecular response properties from the quasi-energy obtained using the variational principle. In Sec. III A, we parametrize the regular wave function and transform the quasi-energy to the frequency domain. In Sec. III B, molecular response properties are determined as derivatives of the quasi-energy with respect to perturbation strengths. In Sec. III C, we apply this development to the determination of molecular response functions for a configuration interaction wave function. In Sec. III D, we show that the definition of molecular response properties as derivatives of the quasi-energy with respect to perturbation strengths is identical to the standard definition based on a Fourier component expansion of the expectation value of an operator representing an observable.

To simplify the notation, we suppress in the remainder of this article the explicit time and perturbation argument, such that, for example, $H(t, \epsilon)$ and $|0(t, \epsilon)\rangle$ are denoted as $H$ and $|0\rangle$, respectively, and the perturbation-dependent quasi-energy $\mathcal{E}(\epsilon)$ is denoted as $\mathcal{E}$.

\section{A. Transformation of the quasi-energy from the time domain to the frequency domain}

In Subsection III A 1, the quasi-energy is determined in the time domain. In Subsection III A 2, the time-dependent wave function coefficients are transformed to the frequency domain and an order expansion in the perturbation strength is introduced for the wave function coefficients in the frequency domain. The quasi-energy is determined in the frequency domain in Subsection III A 3.

\section{Parametrization of the quasi-energy in the time domain}

We now consider the parametrization of the regular wave function, $\left|0_{R}(t, \epsilon)\right\rangle$ of Eq. (23), in the time domain, where the reference state $\left|s_{0}\right\rangle$ of Sec. II B is chosen as an eigenstate $\left|0_{0}\right\rangle$ of the unperturbed system with the energy $E_{0}$,

$$
\begin{aligned}
\left|s_{0}\right\rangle & =\left|0_{0}\right\rangle, \\
H_{0}\left|0_{0}\right\rangle & =E_{0}\left|0_{0}\right\rangle .
\end{aligned}
$$

The states $\left|0_{m}\right\rangle, m \neq 0$ form an orthogonal complement set to $\left|0_{0}\right\rangle$ and are not necessarily eigenstates of $H_{0}$. The regular wave function, $\left|0_{R}(t, \epsilon)\right\rangle$ of Eq. (23), then becomes

$$
\left|0_{R}(t, \epsilon)\right\rangle=N(t, \epsilon)\left|0_{I}(t, \epsilon)\right\rangle,
$$

where we have introduced the intermediate-normalized state

$$
\begin{gathered}
\left|0_{I}(t, \epsilon)\right\rangle=\left|0_{0}\right\rangle+\left|\delta 0_{0}\right\rangle, \\
\left|\delta 0_{0}\right\rangle=\sum_{m \neq 0} b_{m}(t, \epsilon)\left|0_{m}\right\rangle
\end{gathered}
$$

and the normalization constant

$$
N(t, \epsilon)=\left\langle 0_{I}(t, \epsilon) \mid 0_{I}(t, \epsilon)\right\rangle^{-1 / 2}=\left(1+\left\langle\delta 0_{0} \mid \delta 0_{0}\right\rangle\right)^{-1 / 2}
$$

$$
=\left(1+\sum_{m}\left|b_{m}(t, \epsilon)\right|^{2}\right)^{-1 / 2},
$$

since Eq. (60) is satisfied. The unperturbed ground state $\left|0_{0}\right\rangle$ may be determined using the time-independent variational principle of Eq. (57). In the absence of the perturbation $\left|0_{R}(t, \epsilon)\right\rangle$ becomes $\left|0_{0}\right\rangle$, so $\left|\delta 0_{0}\right\rangle$ and thereby $b_{m}(t, \epsilon)$ vanish.

Inserting Eq. (88) into the quasi-energy expression of Eq. (55) gives

$$
\begin{aligned}
\mathcal{E}= & \left\{N^{2}(t, \epsilon)\left\langle 0_{I}(t, \epsilon)\left|H(t, \epsilon)-i \frac{\partial}{\partial t}\right| 0_{I}(t, \epsilon)\right\rangle\right\}_{T} \\
& -i\left\{N(t, \epsilon) \dot{N}(t, \epsilon)\left\langle 0_{I}(t, \epsilon) \mid 0_{I}(t, \epsilon)\right\rangle\right\}_{T} .
\end{aligned}
$$

Using Eq. (90), the second term on the right-hand side of Eq. (91) becomes

$$
\begin{aligned}
& i\left\{N(t, \epsilon) \dot{N}(t, \epsilon)\left\langle 0_{I}(t, \epsilon) \mid 0_{I}(t, \epsilon)\right\rangle\right\}_{T} \\
& \quad=i\left\{N^{-1}(t, \epsilon) \dot{N}(t, \epsilon)\right\}_{T}=i\left\{\frac{\mathrm{d}}{\mathrm{d} t} \ln N(t, \epsilon)\right\}_{T}
\end{aligned}
$$

and vanishes

$$
i\left\{\frac{\mathrm{d}}{\mathrm{d} t} \ln N(t, \epsilon)\right\}_{T}=0,
$$

as follows from Eq. (37) and the periodicity of $N(t, \epsilon)$. The quasi-energy of Eq. (91) therefore becomes

$$
\begin{aligned}
\mathcal{E}= & \left\{N^{2}\left\langle 0_{I}\left|H_{0}\right| 0_{I}\right\rangle\right\}_{T}-i\left\{N^{2}\left\langle 0_{I} \mid \frac{\partial 0_{I}}{\partial t}\right\rangle\right\}_{T} \\
& +\sum_{j_{1}}\left\{N^{2}\left\langle 0_{I}\left|X_{j_{1}}\right| 0_{I}\right\rangle \exp \left(-i \omega_{X_{j_{1}}} t\right)\right\}_{T} \epsilon_{X_{j_{1}}}\left(\omega_{X_{j_{1}}}\right),
\end{aligned}
$$

where we have used Eqs. (2) and (3). The normalization constant $N^{2}$ may be expressed as

$$
\begin{aligned}
N^{2} & =\left(1+\sum_{m}\left|b_{m}(t, \epsilon)\right|^{2}\right)^{-1} \\
& =\sum_{k=0}^{\infty}(-1)^{k}\left(\sum_{m}\left|b_{m}(t, \epsilon)\right|^{2}\right)^{k},
\end{aligned}
$$

where we have used

$$
\frac{1}{1+x}=\sum_{k=0}^{\infty}(-1)^{k} x^{k}
$$

The quasi-energy in Eq. (94) may be written in the form

$$
\mathcal{E}=\mathcal{E}_{H_{0}}+\mathcal{E}_{F}+\sum_{j_{1}} \mathcal{E}_{X_{j_{1}}} \epsilon_{X_{j_{1}}}\left(\omega_{X_{j_{1}}}\right),
$$

where

$$
\begin{aligned}
\mathcal{E}_{H_{0}} & =\left\{N^{2}\left\langle 0_{I}\left|H_{0}\right| 0_{I}\right\rangle\right\}_{T}, \\
\mathcal{E}_{F} & =-i\left\{N^{2}\left\langle 0_{I} \mid \frac{\partial 0_{I}}{\partial t}\right\rangle\right\}_{T}, \\
\mathcal{E}_{X_{j_{1}}}=\mathcal{E}_{X_{j_{1}}}\left(\omega_{X_{j_{1}}}\right) & =\left\{N^{2}\left\langle 0_{I}\left|X_{j_{1}}\right| 0_{I}\right\rangle \exp \left(-i \omega_{X_{j_{1}}} t\right)\right\}_{T} .
\end{aligned}
$$

As we shall see later, the $\mathcal{E}_{F}$ contribution introduces the frequency dependence to the response functions and hence is labeled with $F$. 
Using Eq. (89a), we may write $\mathcal{E}_{H_{0}}$ [Eq. (98)] as

$$
\begin{aligned}
\mathcal{E}_{H_{0}}= & \left\{N ^ { 2 } \left(\left\langle 0_{0}\left|H_{0}\right| 0_{0}\right\rangle+\left\langle 0_{0}\left|H_{0}\right| \delta 0_{0}\right\rangle\right.\right. \\
& \left.\left.+\left\langle\delta 0_{0}\left|H_{0}\right| 0_{0}\right\rangle+\left\langle\delta 0_{0}\left|H_{0}\right| \delta 0_{0}\right\rangle\right)\right\}_{T},
\end{aligned}
$$

which becomes

$$
\mathcal{E}_{H_{0}}=\left\{N^{2}\left(E_{0}+\left\langle\delta 0_{0}\left|H_{0}\right| \delta 0_{0}\right\rangle\right)\right\}_{T},
$$

where we have used that Eq. (57) is satisfied in the limit of vanishing perturbation. Using Eqs. (89b) and (95) and writing out explicitly terms through fourth order in $b_{m}(t, \epsilon)$, Eq. (102) becomes

$$
\begin{aligned}
\mathcal{E}_{H_{0}}= & E_{0}+\sum_{m n}\left\{\left[b_{m}^{*}(t, \epsilon) b_{n}(t, \epsilon)\right.\right. \\
& \left.-\left(\sum_{k} b_{k}^{*}(t, \epsilon) b_{k}(t, \epsilon)\right) b_{m}^{*}(t, \epsilon) b_{n}(t, \epsilon)\right] \\
& \left.\times\left[\left\langle 0_{m}\left|H_{0}\right| 0_{n}\right\rangle-E_{0} \delta_{m n}\right]\right\}_{T}+O\left(b^{6}\right) .
\end{aligned}
$$

Using Eq. (89) and the orthonormality of the $\left\{\left|0_{0}\right\rangle,\left|0_{i}\right\rangle\right\}$ set, the $\mathcal{E}_{F}$ term [Eq. (99)] may be simplified as

$$
\begin{aligned}
\mathcal{E}_{F} & =-i\left\{N^{2}\left\langle\delta 0_{0} \mid \frac{\partial \delta 0_{0}}{\partial t}\right\rangle\right\}_{T} \\
& =-i\left\{N^{2}\left(\sum_{m} b_{m}^{*}(t, \epsilon)\left\langle 0_{m}\right|\right) \sum_{k} \dot{b}_{k}(t, \epsilon)\left|0_{k}\right\rangle\right\}_{T} \\
& =-i\left\{N^{2} \sum_{m} b_{m}^{*}(t, \epsilon) \dot{b}_{m}(t, \epsilon)\right\}_{T},
\end{aligned}
$$

which, using Eq. (95) and keeping terms through fourth order in $b_{m}(t, \epsilon)$, becomes

$$
\begin{aligned}
\mathcal{E}_{F}= & -i \sum_{m}\left\{b_{m}^{*}(t, \epsilon) \dot{b}_{m}(t, \epsilon)\right\}_{T} \\
& +i \sum_{k m}\left\{b_{k}^{*}(t, \epsilon) b_{k}(t, \epsilon) b_{m}^{*}(t, \epsilon) \dot{b}_{m}(t, \epsilon)\right\}_{T}+O\left(b^{6}\right) .
\end{aligned}
$$

For $\mathcal{E}_{X_{j_{1}}}$ [Eq. (100)], we obtain

$$
\begin{aligned}
\mathcal{E}_{X_{j_{1}}}= & \left\{N^{2}\left(\left\langle 0_{0}\left|X_{j_{1}}\right| 0_{0}\right\rangle+\left\langle\delta 0_{0}\left|X_{j_{1}}\right| \delta 0_{0}\right\rangle\right) \exp \left(-i \omega_{X_{j_{1}}} t\right)\right\}_{T} \\
& +\left\{N^{2}\left\langle 0_{0}\left|X_{j_{1}}\right| \delta 0_{0}\right\rangle \exp \left(-i \omega_{X_{j_{1}}} t\right)\right\}_{T} \\
& +\left\{N^{2}\left\langle\delta 0_{0}\left|X_{j_{1}}\right| 0_{0}\right\rangle \exp \left(-i \omega_{X_{j_{1}}} t\right)\right\}_{T}
\end{aligned}
$$

which through third order in $b_{m}(t, \epsilon)$ may be expressed as

$$
\begin{aligned}
\mathcal{E}_{X_{j_{1}}}= & \left\langle 0_{0}\left|X_{j_{1}}\right| 0_{0}\right\rangle\left\{\exp \left(-i \omega_{X_{j_{1}}} t\right)\right\}_{T} \\
& +\sum_{m n}\left\{b_{m}^{*}(t, \epsilon) b_{n}(t, \epsilon) \exp \left(-i \omega_{X_{j_{1}}} t\right)\right\}_{T} \\
& \times\left(\left\langle 0_{m}\left|X_{j_{1}}\right| 0_{n}\right\rangle-\left\langle 0_{0}\left|X_{j_{1}}\right| 0_{0}\right\rangle \delta_{m n}\right) \\
& +\sum_{m}\left[\left\{b_{m}(t, \epsilon) \exp \left(-i \omega_{X_{j_{1}}} t\right)\right\}_{T}\right.
\end{aligned}
$$

$$
\begin{aligned}
& \left.-\sum_{k}\left\{b_{k}^{*}(t, \epsilon) b_{k}(t, \epsilon) b_{m}(t, \epsilon) \exp \left(-i \omega_{X_{j_{1}}} t\right)\right\}_{T}\right] \\
& \times\left\langle 0_{0}\left|X_{j_{1}}\right| 0_{m}\right\rangle+\sum_{m}\left[\left\{b_{m}^{*}(t, \epsilon) \exp \left(-i \omega_{X_{j_{1}}} t\right)\right\}_{T}\right. \\
& \left.-\sum_{k}\left\{b_{k}^{*}(t, \epsilon) b_{k}(t, \epsilon) b_{m}^{*}(t, \epsilon) \exp \left(-i \omega_{X_{j_{1}}} t\right)\right\}_{T}\right] \\
& \times\left\langle 0_{m}\left|X_{j_{1}}\right| 0_{0}\right\rangle+O\left(b^{5}\right) .
\end{aligned}
$$

In order to determine an order expansion of the quasienergy in the perturbation, we now transform the quasi-energy from the time to the frequency domain. In Sec. III A 2, the expansion coefficients of Eq. (89) are transformed from the time to the frequency domain, and in Sec. III A 3, this expansion is used to express the quasi-energy in the frequency domain.

\section{Perturbation expansion of time-dependent wave function coefficients in the frequency domain}

We will now consider a perturbation expansion of the time-dependent expansion coefficients $b_{k}(t, \epsilon)$ of Eq. (89b) in frequency domain with the frequencies satisfying Eq. (7). To simplify the notation, we suppress in the following the explicit reference to the perturbation strength $\epsilon$ for the coefficients,

$$
b_{k}(t)=b_{k}(t, \epsilon)
$$

and express the perturbation expansion of the time-dependent coefficients $b_{k}(t)$ as

$b_{k}(t)=b_{k}^{(0)}+b_{k}^{(1)}(t)+b_{k}^{(2)}(t)+\cdots+b_{k}^{(n)}(t)+\cdots$,

where

$$
\begin{aligned}
b_{k}^{(1)}(t)= & \sum_{j_{1}} b_{k}^{X_{j_{1}}}\left(\omega_{X_{j_{1}}}\right) \epsilon_{X_{j_{1}}}\left(\omega_{X_{j_{1}}}\right) \exp \left(-i \omega_{X_{j_{1}}} t\right), \\
b_{k}^{(2)}(t)= & \frac{1}{2} \sum_{j_{1}} \sum_{j_{2}} b_{k}^{X_{j_{1}} X_{j_{2}}}\left(\omega_{X_{j_{1}}}, \omega_{X_{j_{2}}}\right) \epsilon_{X_{j_{1}}}\left(\omega_{X_{j_{1}}}\right) \\
& \times \epsilon_{X_{j_{2}}}\left(\omega_{X_{j_{2}}}\right) \exp \left(-i\left(\omega_{X_{j_{1}}}+\omega_{X_{j_{2}}}\right) t\right), \\
\vdots & \\
b_{k}^{(n)}(t)= & \frac{1}{n !} \sum_{j_{1}} \ldots \sum_{j_{n}} b_{k}^{X_{j_{1}} \ldots X_{j_{n}}}\left(\omega_{X_{j_{1}}}, \ldots, \omega_{X_{j_{n}}}\right) \\
& \times\left[\prod_{m=1}^{n} \epsilon_{X_{j_{m}}}\left(\omega_{X_{j_{m}}}\right)\right] \exp \left(-i \sum_{m=1}^{n} \omega_{X_{j_{m}}} t\right),
\end{aligned}
$$

and $b_{k}^{(0)}$ are the parameters that are determined in the absence of the perturbation. The $b_{k}^{(0)}$ parameter vanishes since $b_{k}(t)$ is zero in the absence of the perturbation. However, we will later use an expansion of the form of Eqs. (109)-(112) for other quantities that have non-vanishing zero-order terms and we therefore have kept the full expansion in Eq. (109). 
The wave function parameter responses to a given order in the perturbation are given as

$b_{k}^{X_{j_{1} \cdots X_{j_{n}}}}\left(\omega_{X_{j_{1}}}, \ldots, \omega_{X_{j_{n}}}\right)=\left(\frac{\mathrm{d}^{n} b_{k}(t)}{\mathrm{d} \epsilon_{X_{j_{1}}}\left(\omega_{X_{j_{1}}}\right) \cdots \mathrm{d} \epsilon_{X_{j_{n}}}\left(\omega_{X_{j_{n}}}\right)}\right)_{0}$

and are symmetric in the perturbation operator-frequency pairs.

Alternatively, the terms in the expansion of the timedependent coefficients [Eq. (109)] may be collected in a perturbation series which reflects more directly the connection to the frequency domain,

$$
b_{k}(t)=\sum_{K} b_{k}\left(\omega_{K}\right) \exp \left(-i \omega_{K} t\right)
$$

where

$$
\begin{aligned}
b_{k}\left(\omega_{K}\right)= & b_{k}^{(0)} \Delta\left(0-\omega_{K}\right) \\
& +\sum_{j_{1}} b_{k}^{X_{j_{1}}}\left(\omega_{X_{j_{1}}}\right) \epsilon_{X_{j_{1}}}\left(\omega_{X_{j_{1}}}\right) \Delta\left(\omega_{X_{j_{1}}}-\omega_{K}\right) \\
& +\frac{1}{2} \sum_{j_{1} j_{2}} b_{k}^{X_{j_{1}} X_{j_{2}}}\left(\omega_{X_{j_{1}}}, \omega_{X_{j_{2}}}\right) \epsilon_{X_{j_{1}}}\left(\omega_{X_{j_{1}}}\right) \\
& \times \epsilon_{X_{j_{2}}}\left(\omega_{X_{j_{2}}}\right) \Delta\left(\omega_{X_{j_{1}}}+\omega_{X_{j_{2}}}-\omega_{K}\right) \\
& +\ldots \\
& +\frac{1}{n !} \sum_{j_{1} \cdots j_{n}} b_{k}^{X_{j_{1}} \cdots X_{j_{n}}}\left(\omega_{X_{j_{1}}}, \ldots, \omega_{X_{j_{n}}}\right) \\
& \times\left[\prod_{m=1}^{n} \epsilon_{X_{j_{m}}}\left(\omega_{X_{j_{m}}}\right)\right] \Delta\left(\sum_{m=1}^{n} \omega_{X_{j_{m}}}-\omega_{K}\right) \\
& +\ldots
\end{aligned}
$$

and

$$
\Delta(x)=\left\{\begin{array}{ll}
0, & x \neq 0 \\
1, & x=0
\end{array} .\right.
$$

The frequency $\omega_{K}$ thus only gives a non-vanishing contribution, when it is equal to a sum of perturbation frequencies.

The $b_{k}^{*}(t)$ coefficients may be expressed by taking the complex conjugate of Eq. (114),

$$
b_{k}^{*}(t)=\sum_{K} b_{k}^{*}\left(\omega_{K}\right) \exp \left(i \omega_{K} t\right)
$$

where the coefficients $b_{k}^{*}\left(\omega_{K}\right)$ are the complex conjugates of $b_{k}\left(\omega_{K}\right)$ of Eq. (115),

$$
\begin{aligned}
b_{k}^{*}\left(\omega_{K}\right)= & b_{k}^{(0)^{*}} \Delta\left(0+\omega_{K}\right) \\
& +\sum_{j_{1}} b_{k}^{X_{j_{1}}{ }^{*}}\left(-\omega_{X_{j_{1}}}\right) \epsilon_{X_{j_{1}}}\left(\omega_{X_{j_{1}}}\right) \Delta\left(\omega_{X_{j_{1}}}+\omega_{K}\right) \\
& +\frac{1}{2 !} \sum_{j_{1} j_{2}} b_{k}^{X_{j_{1}} X_{j_{2}}{ }^{*}}\left(-\omega_{X_{j_{1}}},-\omega_{X_{j_{2}}}\right) \\
& \times \epsilon_{X_{j_{1}}}\left(\omega_{X_{j_{1}}}\right) \epsilon_{X_{j_{2}}}\left(\omega_{X_{j_{2}}}\right) \Delta\left(\omega_{X_{j_{1}}}+\omega_{X_{j_{2}}}+\omega_{K}\right) \\
& +\cdots \\
& +\frac{1}{n !} \sum_{j_{1} \cdots j_{n}} b_{k}^{X_{j_{1} \cdots X_{j_{n}}}{ }^{*}}\left(-\omega_{X_{j_{1}}}, \ldots,-\omega_{X_{j_{n}}}\right)
\end{aligned}
$$

$$
\begin{aligned}
& \times\left(\prod_{m=1}^{n} \epsilon_{X_{j m}}\left(\omega_{X_{j m}}\right)\right) \Delta\left(\sum_{m=1}^{n} \omega_{X_{j m}}+\omega_{K}\right) \\
& +\cdots,
\end{aligned}
$$

where we have used Eq. (4) and that $\Delta(x)=\Delta(-x)$ and that for each summation index $j_{p}$ both $\epsilon_{X_{j_{p}}}\left(\omega_{X_{j_{p}}}\right)$ and $\epsilon_{X_{j_{p}}}\left(-\omega_{X_{j_{p}}}\right)$ enter the expansion.

The time derivative of the expansion coefficients, $\dot{b}_{k}(t)$, enters in Eq. (105) and may be expressed as

$$
\begin{aligned}
\dot{b}_{k}(t) & =\frac{\mathrm{d}}{\mathrm{d} t} \sum_{K} b_{k}\left(\omega_{K}\right) \exp \left(-i \omega_{K} t\right) \\
& =-i \sum_{K} \omega_{K} b_{k}\left(\omega_{K}\right) \exp \left(-i \omega_{K} t\right) .
\end{aligned}
$$

\section{The quasi-energy in frequency domain}

In the following, we transform the individual terms, $\mathcal{E}_{H_{0}}$ [Eq. (103)], $\mathcal{E}_{F}$ [Eq. (105)], and $\mathcal{E}_{X_{j_{1}}}$ [Eq. (107)], of the quasienergy $\mathcal{E}$ to the frequency domain where the explicit reference to time can be removed. This requires that the time averaging is carried out in Eqs. (103), (105), and (107). In order to do this, we describe initially how the time averaging may be carried out for an exponential operator containing the frequencies of Eq. (7). Then, we determine the quasi-energy in the frequency domain.

We start out showing that the time average of the exponential satisfies

$$
\begin{gathered}
\left\{\exp \left(-i\left[\left(\sum_{K} \omega_{K}-\sum_{L} \omega_{L}\right)+\left(\sum_{m} \omega_{X_{j_{m}}}-\sum_{n} \omega_{X_{j_{n}}}\right)\right] t\right)\right\}_{T} \\
=\Delta\left(\left(\sum_{K} \omega_{K}-\sum_{L} \omega_{L}\right)+\left(\sum_{m} \omega_{X_{j_{m}}}-\sum_{n} \omega_{X_{j_{n}}}\right)\right),
\end{gathered}
$$

where $\omega_{K}$ and $\omega_{L}$ refer to the frequencies of the wave function parameters in Eq. (114) (where $\omega_{K}=n_{K} \omega_{T}$ and $n_{K}$ is an integer) and where $\omega_{X_{j m}}$ and $\omega_{X_{j n}}$ are defined by Eq. (7). To prove Eq. (120), we note that the exponent on the left-hand side of Eq. (120) may be expressed as

$$
\begin{aligned}
\left(\sum_{K} \omega_{K}-\sum_{L} \omega_{L}\right)+\left(\sum_{m} \omega_{X_{j_{m}}}-\sum_{n} \omega_{X_{j_{n}}}\right) \\
=\left(\sum_{K} n_{K} \omega_{T}-\sum_{L} n_{L} \omega_{T}\right) \\
\quad+\left(\sum_{m} n_{X_{j_{m}}} \omega_{T}-\sum_{n} n_{X_{j_{n}}} \omega_{T}\right)=\sum_{q} n_{q} \omega_{T},
\end{aligned}
$$

where $n_{q}$ is an integer that may be positive or negative. Inserting Eq. (121) in the left-hand side of Eq. (120) and assuming $\sum_{q} n_{q} \omega_{T} \neq 0$ gives

$$
\begin{aligned}
\left\{\exp \left(-i \sum_{q} n_{q} \omega_{T} t\right)\right\}_{T} \\
=\frac{1}{-i \sum_{q} n_{q} \omega_{T}}\left\{\frac{\mathrm{d}}{\mathrm{d} t} \exp \left(-i \sum_{q} n_{q} \omega_{T} t\right)\right\}_{T}=0,
\end{aligned}
$$


where we have used Eq. (37). For $\sum_{q} n_{q} \omega_{T}=0$, we obtain

$$
\left\{\exp \left(-i \sum_{q} n_{q} \omega_{T} t\right)\right\}_{T}=1, \quad \sum_{q} n_{q} \omega_{T}=0 .
$$

Combining Eqs. (121)-(123) gives Eq. (120).

We now express the individual terms, $\mathcal{E}_{H_{0}}$ [Eq. (103)], $\mathcal{E}_{F}$ [Eq. (105)], and $\mathcal{E}_{X_{j_{1}}}$ [Eq. (107)], of the quasi-energy $\mathcal{E}$ [Eq. (97)] in the frequency domain removing the explicit reference to time. Inserting Eq. (114) in Eq. (103) and keeping terms through fourth order in the perturbation strength, $\mathcal{E}_{H_{0}}$ becomes

$$
\begin{aligned}
\mathcal{E}_{H_{0}}= & E_{0}+\sum_{m n} \sum_{N}\left[\left(\sum_{M} b_{m}^{*}\left(\omega_{M}\right) b_{n}\left(\omega_{N}\right)\right.\right. \\
& \times\left\{\exp \left(-i\left(\omega_{N}-\omega_{M}\right) t\right)\right\}_{T}-\sum_{k} \sum_{K L M} b_{k}^{*}\left(\omega_{K}\right) \\
& \times b_{k}\left(\omega_{L}\right) b_{m}^{*}\left(\omega_{M}\right) b_{n}\left(\omega_{N}\right) \\
& \left.\times\left\{\exp \left(-i\left(\omega_{L}+\omega_{N}-\omega_{K}-\omega_{M}\right) t\right)\right\}_{T}\right) \\
& \left.\times\left(\left\langle 0_{m}\left|H_{0}\right| 0_{n}\right\rangle-E_{0} \delta_{m n}\right)\right]+O\left(\epsilon^{(6)}\right)
\end{aligned}
$$

Using Eq. (120), we remove any reference to time from $\mathcal{E}_{H_{0}}$,

$$
\begin{aligned}
\mathcal{E}_{H_{0}}= & E_{0}+\sum_{m n} \sum_{M N} b_{m}^{*}\left(\omega_{M}\right) b_{n}\left(\omega_{N}\right) \Delta\left(\omega_{N}-\omega_{M}\right) \\
& \times\left[\left\langle 0_{m}\left|H_{0}\right| 0_{n}\right\rangle-E_{0} \delta_{m n}\right] \\
& -\sum_{k m n} \sum_{K L M N} b_{k}^{*}\left(\omega_{K}\right) b_{k}\left(\omega_{L}\right) b_{m}^{*}\left(\omega_{M}\right) b_{n}\left(\omega_{N}\right) \\
& \times \Delta\left(\omega_{L}+\omega_{N}-\omega_{K}-\omega_{M}\right)\left[\left\langle 0_{m}\left|H_{0}\right| 0_{n}\right\rangle-E_{0} \delta_{m n}\right] \\
& +O\left(\epsilon^{(6)}\right) .
\end{aligned}
$$

The wave function coefficients $b_{n}\left(\omega_{N}\right)$ and $b_{m}^{*}\left(\omega_{M}\right)$ may be viewed as independent parameters. Differentiating $\mathcal{E}_{H_{0}}$ [Eq. (125)] with respect to these independent parameters and taking the limit of the zero perturbation strength gives the Jacobian

$$
J_{m n}=\left(\frac{\partial^{2} \mathcal{E}_{H_{0}}}{\partial b_{m}^{*}\left(\omega_{M}\right) \partial b_{n}\left(\omega_{N}\right)}\right)_{0}
$$

which plays a central role in response function theory. Note that the Jacobian is defined in terms of the quasi-energy differentiated with respect to the time-dependent parameters, rather than in terms of the energy differentiated with respect to the time-independent parameters. The eigenvalues of the Jacobian occur at the excitation energies of the unperturbed molecular system, which may be seen by substituting Eq. (125) in Eq. (126) and using that the coefficients $b_{n}\left(\omega_{N}\right)$ and $b_{m}^{*}\left(\omega_{M}\right)$ vanish at the zero perturbation strength, where the frequencies are equal to zero, giving

$$
J_{m n}=\left\langle 0_{m}\left|H_{0}\right| 0_{n}\right\rangle-E_{0} \delta_{m n} .
$$

In the basis where $\left|0_{n}\right\rangle$ is an eigenstate of $H_{0}$ with the eigenvalue $E_{n}$, the Jacobian matrix is therefore diagonal,

$$
J_{m n}=\left(E_{n}-E_{0}\right) \delta_{m n},
$$

with the eigenvalues equal to the excitation energies of the unperturbed system. Inserting Eq. (127) in Eq. (125) gives

$$
\begin{aligned}
\mathcal{E}_{H_{0}}= & E_{0}+\sum_{m n} \sum_{M N} b_{m}^{*}\left(\omega_{M}\right) b_{n}\left(\omega_{N}\right) J_{m n} \Delta\left(\omega_{N}-\omega_{M}\right) \\
& -\sum_{k m n} \sum_{K L M N} b_{k}^{*}\left(\omega_{K}\right) b_{k}\left(\omega_{L}\right) b_{m}^{*}\left(\omega_{M}\right) b_{n}\left(\omega_{N}\right) J_{m n} \\
& \times \Delta\left(\omega_{L}+\omega_{N}-\omega_{K}-\omega_{M}\right) O\left(\epsilon^{(6)}\right) .
\end{aligned}
$$

We consider now $\mathcal{E}_{F}$ of Eq. (105). Inserting Eqs. (114) and (119) into Eq. (105) and keeping terms through fourth order in the perturbation strength gives

$$
\begin{aligned}
\mathcal{E}_{F}= & -\omega_{N} \sum_{m} \sum_{M N} b_{m}^{*}\left(\omega_{M}\right) b_{m}\left(\omega_{N}\right) \Delta\left(\omega_{N}-\omega_{M}\right) \\
& +\omega_{N} \sum_{k m} \sum_{K L M N} b_{k}^{*}\left(\omega_{K}\right) b_{k}\left(\omega_{L}\right) b_{m}^{*}\left(\omega_{M}\right) b_{m}\left(\omega_{N}\right) \\
& \times \Delta\left(\omega_{L}+\omega_{N}-\omega_{K}-\omega_{M}\right) O\left(\epsilon^{(6)}\right)
\end{aligned}
$$

For the derivation of response function in Sec. III C, it is convenient to combine the $\mathcal{E}_{H_{0}}$ [Eq. (129)] and $\mathcal{E}_{F}$ [Eq. (130)] contributions in $\mathcal{E}_{\mathcal{H}}$,

$$
\begin{aligned}
\mathcal{E}_{\mathcal{H}}= & \mathcal{E}_{H_{0}}+\mathcal{E}_{F} \\
= & E_{0}+\sum_{m n} \sum_{M N} b_{m}^{*}\left(\omega_{M}\right) b_{n}\left(\omega_{N}\right)\left[J_{m n}-\omega_{N} \delta_{m n}\right] \\
& \times \Delta\left(\omega_{N}-\omega_{M}\right)-\sum_{k m n} \sum_{K L M N} b_{k}^{*}\left(\omega_{K}\right) b_{k}\left(\omega_{L}\right) \\
& \times b_{m}^{*}\left(\omega_{M}\right) b_{n}\left(\omega_{N}\right)\left[J_{m n}-\omega_{N} \delta_{m n}\right] \\
& \times \Delta\left(\omega_{L}+\omega_{N}-\omega_{K}-\omega_{M}\right)+O\left(\epsilon^{(6)}\right) .
\end{aligned}
$$

Proceeding in a similar way, $\mathcal{E}_{X_{j_{1}}}$ [Eq. (107)] may be expressed in the frequency domain as

$$
\begin{aligned}
\mathcal{E}_{X_{j_{1}}}= & \left\langle 0_{0}\left|X_{j_{1}}\right| 0_{0}\right\rangle \Delta\left(\omega_{X_{j_{1}}}\right)+\sum_{m n} \sum_{M N} b_{m}^{*}\left(\omega_{M}\right) b_{n}\left(\omega_{N}\right)\left[\left\langle 0_{m}\left|X_{j_{1}}\right| 0_{n}\right\rangle-\left\langle 0_{0}\left|X_{j_{1}}\right| 0_{0}\right\rangle \delta_{m n}\right] \Delta\left(\omega_{X_{j_{1}}}+\omega_{N}-\omega_{M}\right) \\
& +\sum_{m}\left[\sum_{M} b_{m}\left(\omega_{M}\right) \Delta\left(\omega_{X_{j_{1}}}+\omega_{M}\right)-\sum_{k} \sum_{K L M} b_{k}^{*}\left(\omega_{K}\right) b_{k}\left(\omega_{L}\right) b_{m}\left(\omega_{M}\right) \Delta\left(\omega_{X_{j_{1}}}+\omega_{L}-\omega_{K}+\omega_{M}\right)\right]\left\langle 0_{0}\left|X_{j_{1}}\right| 0_{m}\right\rangle \\
& +\sum_{m}\left[\sum_{M} b_{m}^{*}\left(\omega_{M}\right) \Delta\left(\omega_{X_{j_{1}}}-\omega_{M}\right)-\sum_{k} \sum_{K L M} b_{k}^{*}\left(\omega_{K}\right) b_{k}\left(\omega_{L}\right) b_{m}^{*}\left(\omega_{M}\right) \Delta\left(\omega_{X_{j_{1}}}+\omega_{L}-\omega_{K}-\omega_{M}\right)\right]\left\langle 0_{m}\left|X_{j_{1}}\right| 0_{0}\right\rangle \\
& +O\left(\epsilon^{(5)}\right)
\end{aligned}
$$


The quasi-energy in Eq. (97) may thus be written as

$$
\mathcal{E}=\mathcal{E}_{\mathcal{H}}+\sum_{j_{1}} \mathcal{E}_{X_{j_{1}}} \epsilon_{X_{j_{1}}}\left(\omega_{X_{j_{1}}}\right),
$$

where $\mathcal{E}_{\mathcal{H}}$ and $\mathcal{E}_{X_{j_{1}}}$ are given in Eqs. (131) and (132), respectively.

\section{B. Molecular response properties from an order expansion of the quasi-energy in the frequency domain}

In Sec. II F, molecular response functions were identified as expansion coefficients $\mathcal{E}^{X_{j_{1}} \ldots X_{j_{n}}}\left(\omega_{X_{j_{1}}}, \ldots, \omega_{X_{j_{n}}}\right)$ [Eq. (65)] of a Taylor series expansion of the quasi-energy $\mathcal{E}$ in the perturbation strengths [Eq. (64)], where the expansion coefficients were symmetric with respect to the operator-frequency pair permutations in Eq. (66). In Sec. III A, we derived an expansion of the quasi-energy [Eq. (133)] expressed in terms of two contributions: $\mathcal{E}_{\mathcal{H}}$ [Eq. (131)] and $\mathcal{E}_{X_{j_{1}}}$ [Eq. (132)]. From the expansion in Eq. (133), it is not obvious how to rearrange the terms to end up with a Taylor series, where the expansion coefficients satisfy the operator-frequency pair permutational symmetry. In Sec. III B 1, we describe how this rearrangement can be performed. As a byproduct of this derivation, we also show that the frequencies of the expansion coefficients $\mathcal{E}^{X_{j_{1}} \ldots X_{j_{n}}}\left(\omega_{X_{j_{1}}}, \ldots, \omega_{X_{j_{n}}}\right)$ must satisfy $\sum_{m=1}^{n} \omega_{X_{j_{m}}}=0$. In Sec. III B 2, the variational conditions are established for the expansion coefficients.

\section{Order expansion of the quasi-energy in the perturbation strengths}

To establish the rearrangement of the terms in Eq. (133) leading to a Taylor series, we first express Eq. (64) in terms of summation over distinct (i.e., not related by the permutational symmetry) $n$-fold multiplets of the operator-frequency pairs,

$$
\mathcal{E}=\sum_{n=0}^{\infty} \mathcal{E}^{(n)}
$$

where

$$
\begin{aligned}
\mathcal{E}^{(n)}= & \frac{1}{n !} \sum_{j_{1} \leq \cdots \leq j_{n}} \mathcal{N}_{\omega_{X_{j_{1}}} \ldots \omega_{X_{j_{n}}}}^{X_{j_{1}} \ldots X_{j_{n}}} \mathcal{E}^{X_{j_{1}} \ldots X_{j_{n}}}\left(\omega_{X_{j_{1}}}, \ldots, \omega_{X_{j_{n}}}\right) \\
& \times \epsilon_{X_{j_{1}}}\left(\omega_{X_{j_{1}}}\right) \ldots \epsilon_{X_{j_{n}}}\left(\omega_{X_{j_{n}}}\right)
\end{aligned}
$$

and where

$$
\mathcal{N}_{\omega_{X_{j_{1}}} \ldots \omega_{X_{j n}}}^{X_{j_{1}} \ldots X_{j_{n}}}=\frac{n !}{\prod_{k} n_{k} !}
$$

and $n_{k}$ is the number of times the perturbation-frequency pair

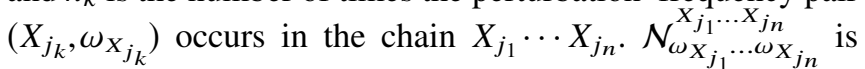
thus the number of distinct permutations of a given multiplet. For example, for a set of two operator-frequency pairs $\left\{\left(Y, \omega_{Y}\right),\left(Z, \omega_{Z}\right)\right\}$, the second-order term $(n=2)$ in Eq. (64) becomes

$$
\begin{aligned}
& \frac{1}{2 !}\left[\left(\frac{\mathrm{d}^{2} \mathcal{E}}{\mathrm{d} \epsilon_{Y} \mathrm{~d} \epsilon_{Y}}\right)_{0} \epsilon_{Y} \epsilon_{Y}+\left(\frac{\mathrm{d}^{2} \mathcal{E}}{\mathrm{d} \epsilon_{Y} \mathrm{~d} \epsilon_{Z}}\right)_{0} \epsilon_{Y} \epsilon_{Z}\right. \\
& \left.\quad+\left(\frac{\mathrm{d}^{2} \mathcal{E}}{\mathrm{d} \epsilon_{Z} \mathrm{~d} \epsilon_{Y}}\right)_{0} \epsilon_{Z} \epsilon_{Y}+\left(\frac{\mathrm{d}^{2} \mathcal{E}}{\mathrm{d} \epsilon_{Z} \mathrm{~d} \epsilon_{Z}}\right)_{0} \epsilon_{Z} \epsilon_{Z}\right]
\end{aligned}
$$

$$
\begin{aligned}
= & \frac{1}{2 !}\left[\left(\frac{\mathrm{d}^{2} \mathcal{E}}{\mathrm{d} \epsilon_{Y} \mathrm{~d} \epsilon_{Y}}\right)_{0} \epsilon_{Y} \epsilon_{Y}+2\left(\frac{\mathrm{d}^{2} \mathcal{E}}{\mathrm{d} \epsilon_{Y} \mathrm{~d} \epsilon_{Z}}\right)_{0} \epsilon_{Y} \epsilon_{Z}\right. \\
& \left.+\left(\frac{\mathrm{d}^{2} \mathcal{E}}{\mathrm{d} \epsilon_{Z} \mathrm{~d} \epsilon_{Z}}\right)_{0} \epsilon_{Z} \epsilon_{Z}\right]
\end{aligned}
$$

since the $Z Y$ and $Y Z$ components are related by permutational symmetry and $\mathcal{N}_{\omega_{Y} \omega_{Z}}^{Y Z}=2$ and $\mathcal{N}_{\omega_{Y} \omega_{Y}}^{Y Y}=\mathcal{N}_{\omega_{Z} \omega_{Z}}^{Z Z}=1$.

We now consider the expansion in Eq. (133). By inserting Eqs. (131) and (132) into Eq. (133) and collecting terms of the same order in the perturbation strength and using Eqs. (114) and (117), the $n$ th-order quasi-energy correction may be written as

$$
\begin{aligned}
\mathcal{E}^{(n)}= & \sum_{j_{1} \cdots j_{n}} f_{\omega_{X_{j_{1}}} \ldots \omega_{X_{j n}}}^{X_{j_{1}} \ldots X_{X_{j}}} \epsilon_{X_{j_{1}}}\left(\omega_{X_{j_{1}}}\right) \ldots \epsilon_{X_{j_{n}}}\left(\omega_{X_{j_{n}}}\right) \\
& \times \Delta\left(\sum_{m=1}^{n} \omega_{X_{j m}}\right)
\end{aligned}
$$

$\mathcal{E}^{(n)}$ contains $n$ perturbation strength parameters, an expansion coefficient $f_{\omega_{X_{j_{1}}} \ldots \omega_{X_{j_{n}}}}^{X_{j_{1}} \ldots X_{j_{n}}}$, and a factor $\Delta\left(\sum_{m=1}^{n} \omega_{X_{j_{m}}}\right)$. The latter originates from the presence of $\Delta\left(\sum_{K} \omega_{K}\right)$ in Eq. (131) and $\Delta\left(\omega_{X_{j_{1}}}+\sum_{K} \omega_{K}\right)$ in Eq. (132), where the frequencies $\omega_{K}$ from $b_{k}\left(\omega_{K}\right)$ in Eq. (115) are resolved according to the frequency constraint $\Delta\left(\sum_{m=1}^{n} \omega_{X_{j m}}-\omega_{K}\right)$ in Eq. (115), and similar for $b_{k}^{*}\left(\omega_{K}\right)$ in Eq. (118).

The expansion coefficients $f_{\omega_{X_{j_{1}}} \ldots \omega_{X_{j_{n}}}}^{X_{j_{1}} \ldots X_{j_{n}}}$ contain matrix elements of the unperturbed Hamiltonian and the perturbation operators multiplied by a sum of products of coefficients $b_{k}^{X_{j_{1}} \cdots X_{j_{l}}}$ (where $l$ runs through all orders up to $n$ ). For example, one of the third-order contributions from $\mathcal{E}_{\mathcal{H}}$ of Eq. (131),

$$
\begin{aligned}
\mathcal{E}^{(3)} \longleftarrow & \sum_{m n} \sum_{M N} b_{m}^{*}\left(\omega_{M}\right) b_{n}\left(\omega_{N}\right)\left[J_{m n}-\omega_{N} \delta_{m n}\right] \\
& \times \Delta\left(\omega_{N}-\omega_{M}\right)
\end{aligned}
$$

has the form

$$
\begin{aligned}
& \mathcal{E}^{(3)} \longleftarrow \frac{1}{2} \sum_{j_{1} j_{2} j_{3}} \sum_{m n} b_{m}^{X_{j_{1}}{ }^{*}}\left(-\omega_{X_{j_{1}}}\right) b_{n}^{X_{j_{2}}{ }^{X_{j_{3}}}}\left(\omega_{X_{j_{2}}}, \omega_{X_{j_{3}}}\right) \\
& \times\left[J_{m n}-\left(\omega_{X_{j_{2}}}+\omega_{X_{j_{3}}}\right) \delta_{m n}\right] \\
& \times \epsilon_{X_{j_{1}}}\left(\omega_{X_{j_{1}}}\right) \epsilon_{X_{j_{2}}}\left(\omega_{X_{j_{2}}}\right) \epsilon_{X_{j_{3}}}\left(\omega_{X_{j_{3}}}\right) \\
& \times \Delta\left(\omega_{X_{j_{1}}}+\omega_{X_{j_{2}}}+\omega_{X_{j_{3}}}\right) \text {, }
\end{aligned}
$$

where the factor $\frac{1}{2}$ arises from the second-order coefficients $b_{m}^{X_{j_{2}} X_{j_{3}}}\left(\omega_{X_{j_{2}}}, \omega_{X_{j_{3}}}\right)$ in Eq. (115). The contribution to

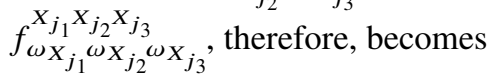

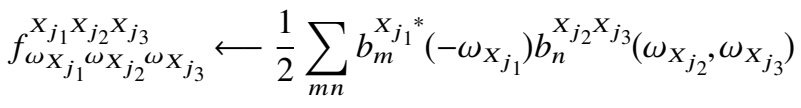

$$
\begin{aligned}
& \times\left[J_{m n}-\left(\omega_{X_{j_{2}}}+\omega_{X_{j_{3}}}\right) \delta_{m n}\right] \text {. }
\end{aligned}
$$

It follows from Eq. (138) that 


$$
\begin{aligned}
\mathcal{E}^{(n)}= & \sum_{j_{1} \cdots j_{n}} f_{\omega_{X_{j_{1}}} \ldots \omega_{X_{j_{n}}}}^{X_{j_{1} \ldots X_{j_{n}}}} \epsilon_{X_{j_{1}}}\left(\omega_{X_{j_{1}}}\right) \ldots \epsilon_{X_{j_{n}}}\left(\omega_{X_{j_{n}}}\right), \\
& \sum_{m=1}^{n} \omega_{X_{j_{m}}}=0
\end{aligned}
$$

where we have introduced the frequency constraint explicitly. We now introduce the permutation operator $P_{\omega_{X_{j_{1}}} \ldots \omega_{X_{j_{n}}}}^{X_{j_{1}} \ldots X_{j_{n}}}$ that generates all $n$ ! (distinct and non-distinct) permutations of the operator-frequency pairs

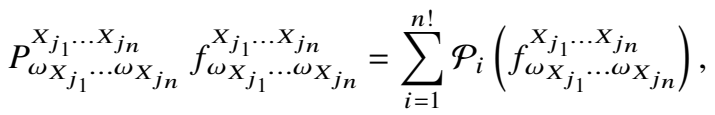

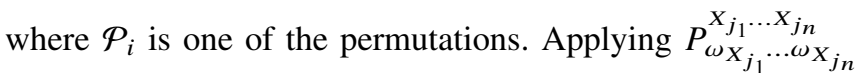
on the right-hand side of Eq. (142) only describes a relabeling of the terms in the summation and we may therefore rewrite Eq. (142) as

$$
\begin{aligned}
& \mathcal{E}^{(n)}=\sum_{j_{1} \cdots j_{n}} \epsilon_{X_{j_{1}}}\left(\omega_{X_{j_{1}}}\right) \ldots \epsilon_{X_{j_{n}}}\left(\omega_{X_{j_{n}}}\right)
\end{aligned}
$$

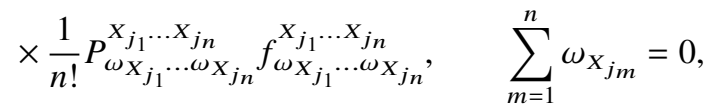

where the factor $\frac{1}{n !}$ has been introduced because the permutation operator generates $n$ ! copies of each term. To identify $\mathcal{E}^{X_{j_{1}} \ldots X_{j_{n}}}\left(\omega_{X_{j_{1}}}, \ldots, \omega_{X_{j_{n}}}\right)$ of Eq. (135), we have to rearrange Eq. (144) to contain only distinct multiplets of

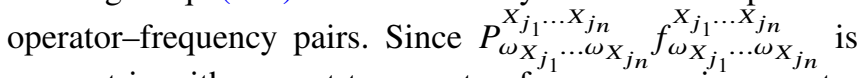
symmetric with respect to operator-frequency pair permutations as is the case for $\mathcal{E}^{X_{j_{1}} \ldots X_{j_{n}}}\left(\omega_{X_{j_{1}}}, \ldots, \omega_{X_{j_{n}}}\right)$, we may in Eq. (144) restrict the summation range introducing the factor $\mathcal{N}_{\omega_{X_{j_{1}}} \ldots \omega_{X_{j_{n}}}}^{X_{j_{1}} \ldots X_{j_{n}}}$, giving

$$
\begin{aligned}
& \mathcal{E}^{(n)}=\sum_{j_{1} \leq \cdots \leq j_{n}} \epsilon_{X_{j_{1}}}\left(\omega_{X_{j_{1}}}\right) \ldots \epsilon_{X_{j_{n}}}\left(\omega_{X_{j_{n}}}\right) \frac{\mathcal{N}_{\omega_{X_{j_{1}}} \cdots \omega_{X_{j_{n}}}}^{X_{j_{1}} \ldots X_{j_{n}}}}{n !}
\end{aligned}
$$

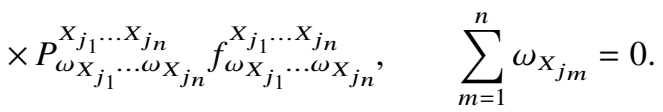

Equating Eqs. (135) and (145), we obtain

$$
\begin{aligned}
& \mathcal{E}^{X_{j_{1}} \ldots X_{j_{n}}}\left(\omega_{X_{j_{1}}}, \ldots, \omega_{X_{j_{n}}}\right)=P_{\omega_{X_{j_{1}}} \ldots \omega_{X_{j_{n}}}}^{X_{j_{1} \ldots X_{j_{n}}}} f_{\omega_{j_{1}} \ldots \omega_{X_{j_{n}}}}^{X_{j_{1}} \ldots X_{j_{n}}}, \\
& \sum_{m=1}^{n} \omega_{X_{j m}}=0 \text {. }
\end{aligned}
$$

Equation (146) shows that $\mathcal{E}^{X_{j_{1}} \ldots X_{j_{n}}}\left(\omega_{X_{j_{1}}}, \ldots, \omega_{X_{j_{n}}}\right)$ may be obtained identifying the symmetry distinct contributions in Eq. (133) and then applying the permutation operator on these contributions. $\mathcal{E}^{X_{j_{1}} \ldots X_{j_{n}}}\left(\omega_{X_{j_{1}}}, \ldots, \omega_{X_{j_{n}}}\right)$ will then be symmetric with respect to the operator-frequency pair permutations in Eq. (66) and satisfy the frequency constraint in Eq. (142).

\section{Stationary conditions for the quasi-energy and its perturbation components}

Let us now consider the stationary conditions for the quasi-energy $\mathcal{E}$ of Eq. (133), where $\mathcal{E}_{\mathcal{H}}$ and $\mathcal{E}_{X_{j_{1}}}$ are defined in Eqs. (131) and (132), respectively, in terms of the frequencydependent coefficients $b_{i}\left(\omega_{K}\right)$. The quasi-energy is variational with respect to all periodic variations of the wave function (see Eq. (58)) and in particular stationary in the coefficients $b_{i}\left(\omega_{K}\right)$ of Eq. (115),

$$
\frac{\partial \mathcal{E}}{\partial b_{i}\left(\omega_{K}\right)}=0, \quad \omega_{K}=n_{K} \omega_{T},
$$

and in the coefficients $b_{i}^{X_{j_{1}} \cdots X_{j_{n}}}\left(\omega_{X_{j_{1}}}, \ldots, \omega_{X_{j_{n}}}\right)$ of Eq. (113),

$$
\frac{\partial \mathcal{E}}{\partial b_{i}^{X_{j_{1} \cdots X_{j_{k}}}}\left(\omega_{X_{j_{1}}}, \ldots, \omega_{X_{j_{k}}}\right)}=0, \quad \sum_{m=1}^{k} \omega_{X_{j_{m}}}=n \omega_{T},
$$

where $n_{K}$ and $n$ are integers. Equations (147) and (148) hold for all values of the perturbation strengths $\epsilon$. Differentiating Eq. (148) with respect to perturbation strengths and setting $\epsilon=0$ gives

$$
\begin{aligned}
& 0=\left(\frac{\mathrm{d}^{n}}{\mathrm{~d} \epsilon_{X_{j_{1}}}\left(\omega_{X_{j_{1}}}\right) \cdots \mathrm{d} \epsilon_{X_{j_{n}}}\left(\omega_{X_{j_{n}}}\right)}\right. \\
& \left.\times \frac{\partial \mathcal{E}}{\partial b_{i}^{X_{j_{1}} \cdots X_{j_{k}}}\left(\omega_{X_{j_{1}}}, \ldots, \omega_{X_{j_{k}}}\right)}\right)_{0}
\end{aligned}
$$

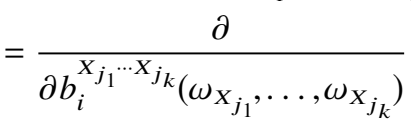

$$
\begin{aligned}
& \times\left(\frac{\mathrm{d}^{n} \mathcal{E}}{\mathrm{d} \epsilon_{X_{j_{1}}}\left(\omega_{X_{j_{1}}}\right) \cdots \mathrm{d} \epsilon_{X_{j_{n}}}\left(\omega_{X_{j_{n}}}\right)}\right) .
\end{aligned}
$$

Using Eqs. (65) and (146), we obtain

$\frac{\partial \mathcal{E}^{X_{j_{1}} \cdots X_{j_{n}}}\left(\omega_{X_{j_{1}}}, \ldots, \omega_{X_{j_{n}}}\right)}{\partial b_{i}^{X_{j_{1} \cdots X_{j_{k}}}}\left(\omega_{X_{j_{1}}}, \ldots, \omega_{X_{j_{k}}}\right)}=0, \quad \sum_{m=1}^{n} \omega_{X_{j_{m}}}=0$.

Since $\mathcal{E}$ is also stationary in the coefficients $b_{i}^{X_{j_{1}} \cdots X_{j_{k}}}{ }^{*}$ $\left(-\omega_{X_{j_{1}}}, \ldots,-\omega_{X_{j_{k}}}\right)$, we obtain in a similar way that

$$
\frac{\partial \mathcal{E}^{X_{j_{1}} \cdots X_{j_{n}}}\left(\omega_{X_{j_{1}}}, \ldots, \omega_{X_{j_{n}}}\right)}{\partial b_{i}^{X_{j_{1}} \cdots X_{j_{k}}{ }^{*}}\left(-\omega_{X_{j_{1}}}, \ldots,-\omega_{X_{j_{k}}}\right)}=0, \quad \sum_{m=1}^{n} \omega_{X_{j_{m}}}=0 .
$$

We thus conclude that the response functions $\mathcal{E}^{X_{j_{1}} \ldots X_{j_{n}}}\left(\omega_{X_{j_{1}}}\right.$, $\left.\ldots, \omega_{X_{j_{n}}}\right)$ are variational in the perturbation components $b_{i}^{X_{j_{1} \cdots X_{j_{k}}}}\left(\omega_{X_{j_{1}}}, \ldots, \omega_{X_{j_{k}}}\right)$ and $b_{i}^{X_{j_{1}} \cdots X_{j_{k}}}{ }^{*}\left(-\omega_{X_{j_{1}}}, \ldots,-\omega_{X_{j_{k}}}\right)$.

\section{Response functions through fourth order}

We now derive explicit expressions for molecular response properties through fourth order. We initially identify $\mathcal{E}^{X_{j_{1}} \ldots X_{j_{n}}}\left(\omega_{X_{j_{1}}}, \ldots, \omega_{X_{j_{n}}}\right)$ by writing $\mathcal{E}^{(n)}$ in terms of the individual contributions from $\mathcal{E}_{\mathcal{H}}$ [Eq. (131)] and $\mathcal{E}_{X_{j_{1}}}$ 
[Eq. (132)],

$$
\mathcal{E}^{(n)}=\mathcal{E}_{\mathcal{H}}^{(n)}+\sum_{j_{1}} \mathcal{E}_{X_{j_{1}}}^{(n-1)}\left(\omega_{X_{j_{1}}}\right) \epsilon_{X_{j_{1}}}\left(\omega_{X_{j_{1}}}\right),
$$

identifying, according to Eq. (146), the individual distinct contributions to $\mathcal{E}^{X_{j_{1}} \cdots X_{j_{n}}}\left(\omega_{X_{j_{1}}}, \ldots, \omega_{X_{j_{n}}}\right)$. We then obtain response equations by differentiating $\mathcal{E}^{X_{j_{1}} \ldots X_{j_{n}}}\left(\omega_{X_{j_{1}}}, \ldots\right.$, $\left.\omega_{X_{j_{n}}}\right)$ with respect to $b_{k}^{X_{j_{1}} \cdots X_{j_{m}}{ }^{*}}\left(-\omega X_{j_{1}, \ldots,}, \omega x_{j_{m}}\right)$ using the stationary condition of Eq. (151). Finally, we determine molecular property expression by simplifying $\mathcal{E}^{X_{j_{1}} \ldots X_{j_{n}}}\left(\omega_{X_{j_{1}}}, \ldots\right.$, $\omega_{X_{j_{n}}}$ ) using the $2 n+1$ rule, see, for example, Ref. 77 .

\section{Energy and first-order molecular properties}

From Eq. (152), we determine $\mathcal{E}^{(0)}$ as

$$
\mathcal{E}^{(0)}=\mathcal{E}_{\mathcal{H}}^{(0)}=\mathcal{E}_{H_{0}}^{(0)}=E_{0}
$$

since the expansion coefficients $b_{i}\left(\omega_{K}\right)$ do not contain zeroorder contributions.

Using Eq. (146), the first-order molecular property expression is obtained as

$$
\mathcal{E}^{X_{j_{1}}}\left(\omega_{X_{j_{1}}}\right)=\left\langle 0_{0}\left|X_{j_{1}}\right| 0_{0}\right\rangle, \quad \omega_{X_{j_{1}}}=0 .
$$

The first-order molecular properties are thus frequencyindependent and expressed in terms of the ground state expectation value of a perturbation operator, as in the timeindependent perturbation theory.

\section{Second-order molecular properties}

Using Eq. (146), the second-order quasi-energy becomes

$$
\begin{aligned}
& \mathcal{E}^{X_{j_{1}} X_{j_{2}}\left(\omega_{X_{j_{1}}}, \omega_{X_{j_{2}}}\right)} \\
& =P_{\omega_{X_{j_{1}}}{ }^{X_{j_{1}} X_{j_{2}}} \sum_{m}}\left[b_{m}^{X_{j_{1}}{ }^{*}}\left(-\omega_{X_{j_{1}}}\right) \sum_{n} b_{n}^{X_{j_{2}}}\left(\omega_{X_{j_{2}}}\right)\right.
\end{aligned}
$$

$$
\begin{aligned}
& \times\left(J_{m n}-\omega_{X_{j_{2}}} \delta_{m n}\right)+b_{m}^{X_{j_{2}}}\left(\omega_{X_{j_{2}}}\right)\left\langle 0_{0}\left|X_{j_{1}}\right| 0_{m}\right\rangle \\
& \left.+b_{m}^{X_{j_{2}}{ }^{*}}\left(-\omega_{X_{j_{2}}}\right)\left\langle 0_{m}\left|X_{j_{1}}\right| 0_{0}\right\rangle\right], \quad \omega_{X_{j_{1}}}+\omega_{X_{j_{2}}}=0,
\end{aligned}
$$

where we have used Eqs. (131) and (132).

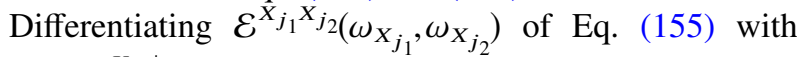
respect to $b_{k}^{X_{j_{2}}{ }^{*}}\left(-\omega_{X_{j_{2}}}\right)$ and using the stationary condition of Eq. (151)

$$
\frac{\partial \mathcal{E}^{X_{j_{1}} X_{j_{2}}\left(\omega_{X_{j_{1}}}, \omega_{X_{j_{2}}}\right)}}{\partial b_{k}^{X_{j_{2}}{ }^{*}}\left(-\omega_{X_{j_{2}}}\right)}=0,
$$

we obtain the first-order amplitude equations

$\sum_{n}\left(J_{k n}-\omega_{X_{j_{1}}} \delta_{k n}\right) b_{n}^{X_{j_{1}}}\left(\omega_{X_{j_{1}}}\right)=-\left\langle 0_{k}\left|X_{j_{1}}\right| 0_{0}\right\rangle$,

from which $b_{n}^{X_{j_{1}}}\left(\omega_{X_{j_{1}}}\right)$ may be calculated.

Using Eq. (157), second-order molecular properties may be simplified as

$$
\begin{aligned}
\mathcal{E}^{X_{j_{1}} X_{j_{2}}\left(\omega_{X_{j_{1}}}, \omega_{X_{j_{2}}}\right)=} & P_{\omega_{X_{j_{1}}} \omega_{X_{j_{2}}}^{X_{j_{2}} X_{j_{2}}} \sum_{m}} b_{m}^{X_{j_{2}}}\left(\omega_{X_{j_{2}}}\right) \\
& \times\left\langle 0_{0}\left|X_{j_{1}}\right| 0_{m}\right\rangle, \quad \omega_{X_{j_{1}}}+\omega_{X_{j_{2}}}=0,
\end{aligned}
$$

where $b_{m}^{X_{j_{2}}}\left(\omega_{X_{j_{2}}}\right)$ are obtained from Eq. (157). This is equivalent to applying the $2 n+1$ rule for even-order corrections to quasi-energy and using that the complex conjugate coefficients satisfy for even-order a $2 n+2$ rule.

\section{Third-order molecular properties}

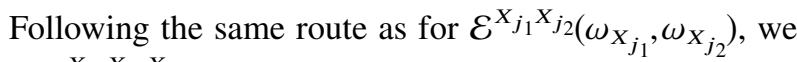

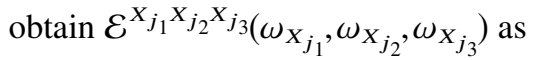

$$
\begin{aligned}
& \mathcal{E}^{X_{j_{1}} X_{j_{2}} X_{j_{3}}\left(\omega_{X_{j_{1}}}, \omega_{X_{j_{2}}}, \omega_{X_{j_{3}}}\right)}
\end{aligned}
$$

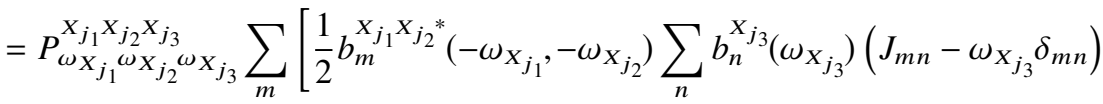

$$
\begin{aligned}
& +\frac{1}{2} b_{m}^{X_{j_{1}}{ }^{*}}\left(-\omega_{X_{j_{1}}}\right) \sum_{n} b_{n}^{X_{j_{2}} X_{j_{3}}}\left(\omega_{X_{j_{2}}}, \omega_{X_{j_{3}}}\right)\left(J_{m n}-\left(\omega_{X_{j_{2}}}+\omega_{X_{j_{3}}}\right) \delta_{m n}\right) \\
& +b_{m}^{X_{j_{2}}{ }^{*}}\left(-\omega_{X_{j_{2}}}\right) \sum_{n} b_{n}^{X_{j_{3}}}\left(\omega_{X_{j_{3}}}\right)\left(\left\langle 0_{m}\left|X_{j_{1}}\right| 0_{n}\right\rangle-\left\langle 0_{0}\left|X_{j_{1}}\right| 0_{0}\right\rangle \delta_{m n}\right) \\
& \left.+\frac{1}{2}\left(b_{m}^{X_{j_{2}} X_{j_{3}}}\left(\omega_{X_{j_{2}}}, \omega_{X_{j_{3}}}\right)\left\langle 0_{0}\left|X_{j_{1}}\right| 0_{m}\right\rangle+b_{m}^{X_{j_{2}} X_{j_{3}}{ }^{*}}\left(-\omega_{X_{j_{2}}},-\omega_{X_{j_{3}}}\right)\left\langle 0_{m}\left|X_{j_{1}}\right| 0_{0}\right\rangle\right)\right], \quad \omega_{X_{j_{1}}}+\omega_{X_{j_{2}}}+\omega_{X_{j_{3}}}=0 .
\end{aligned}
$$

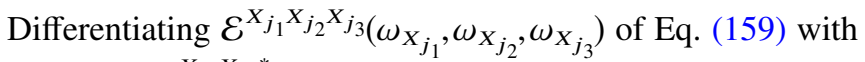

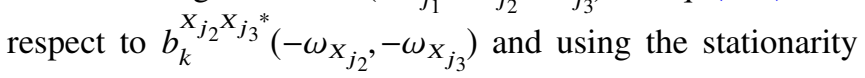

of $\mathcal{E}^{X_{j_{1}} X_{j_{2}} X_{j_{3}}}\left(\omega_{X_{j_{1}}}, \omega_{X_{j_{2}}}, \omega_{X_{j_{3}}}\right)$, we recover the first-order response equation of Eq. (157). Differentiating with respect to 
$b_{k}^{X_{j_{3}}{ }^{*}}\left(-\omega_{X_{j_{3}}}\right)$, we obtain the second-order response equation

$$
\begin{aligned}
& \sum_{n}\left(J_{k n}-\left(\omega_{X_{j_{1}}}+\omega_{X_{j_{2}}}\right) \delta_{k n}\right) b_{n}^{X_{j_{1}} X_{j_{2}}}\left(\omega_{X_{j_{1}}}, \omega_{X_{j_{2}}}\right) \\
& =-P_{\omega_{X_{j_{1}}}{ }_{\boldsymbol{J}_{X_{j}} X_{j_{2}}}}^{X_{j_{2}}} \sum_{n} b_{n}^{X_{j_{2}}}\left(\omega_{X_{j_{2}}}\right) \\
& \times\left(\left\langle 0_{k}\left|X_{j_{1}}\right| 0_{n}\right\rangle-\left\langle 0_{0}\left|X_{j_{1}}\right| 0_{0}\right\rangle \delta_{k n}\right),
\end{aligned}
$$

from which $b_{n}^{X_{j_{1}} X_{j_{2}}}\left(\omega_{X_{j_{1}}}, \omega_{X_{j_{2}}}\right)$, needed in Subsection III C 4 for the fourth-order properties, may be calculated.
Using the $2 n+1$ rule, third-order molecular properties become

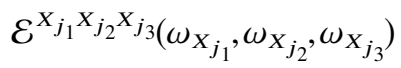

$$
\begin{aligned}
& =P_{\omega_{X_{j_{1}}} \omega_{X_{j_{2}}} X_{j_{1}} X_{j_{j_{3}}} X_{j_{3}}} \sum_{m n} b_{m}^{X_{j_{2}}{ }^{*}}\left(-\omega_{X_{j_{2}}}\right) b_{n}^{X_{j_{3}}}\left(\omega_{X_{j_{3}}}\right) \\
& \times\left(\left\langle 0_{m}\left|X_{j_{1}}\right| 0_{n}\right\rangle-\left\langle 0_{0}\left|X_{j_{1}}\right| 0_{0}\right\rangle \delta_{m n}\right), \\
& \omega_{X_{j_{1}}}+\omega_{X_{j_{2}}}+\omega_{X_{j_{3}}}=0 \text {, }
\end{aligned}
$$

where $b_{n}^{X_{j_{3}}}\left(\omega_{X_{j_{3}}}\right)$ are obtained from Eq. (157) and $b_{m}^{X_{j_{2}}{ }^{*}}\left(-\omega_{X_{j_{2}}}\right)$ from the complex conjugate of Eq. (157).

\section{Fourth-order molecular properties}

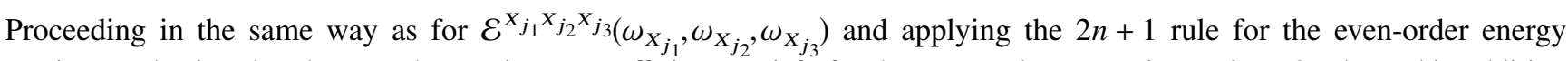
corrections and using that the complex conjugate coefficients satisfy for the even-order corrections a $2 n+2$ rule, and in addition applying the first-order equation [Eq. (157)], the fourth-order molecular property expression becomes

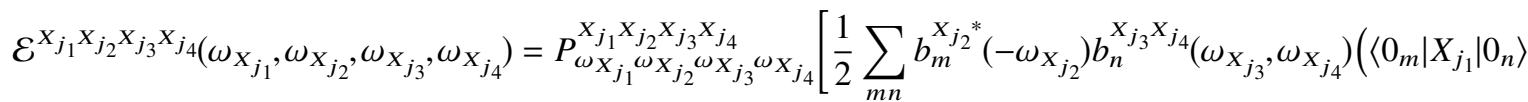

$$
\begin{aligned}
& \left.\left.-\left\langle 0_{0}\left|X_{j_{1}}\right| 0_{0}\right\rangle \delta_{m n}\right)-\sum_{k} b_{k}^{X_{j_{2}}{ }^{*}}\left(-\omega_{X_{j_{2}}}\right) b_{k}^{X_{j_{3}}}\left(\omega_{X_{j_{3}}}\right) \sum_{m} b_{m}^{X_{j_{4}}}\left(\omega_{X_{j_{4}}}\right)\left\langle 0_{0}\left|X_{j_{1}}\right| 0_{m}\right\rangle\right], \\
& \omega_{X_{j_{1}}}+\omega_{X_{j_{2}}}+\omega_{X_{j_{3}}}+\omega_{X_{j_{4}}}=0 \text {. }
\end{aligned}
$$

where $b_{m}^{X_{j_{4}}}\left(\omega_{X_{j_{4}}}\right)$ are obtained from Eq. (157), $b_{m}^{X_{j_{2}}{ }^{*}}\left(-\omega_{X_{j_{2}}}\right)$ from the complex conjugate of Eq. (157), and $b_{n}^{X_{j_{2}} X_{j_{3}}}$ $\left(\omega_{X_{j_{2}}}, \omega_{X_{j_{3}}}\right)$ from Eq. (160).

The derivation presented in this section may be extended to any order.

\section{Molecular properties from the quasi-energy compared to the standard definition}

We have in this article identified molecular response properties as derivatives of the quasi-energy with respect to perturbation strengths. Traditionally, molecular response properties have been defined as the expansion coefficients of a perturbation expansion of the expectation value of an observable, $\left.\widetilde{\langle 0}\left|X_{i}\right| \widetilde{0}\right\rangle$, for the time-dependent wave function $|\widetilde{0}\rangle$. Since $|\widetilde{0}\rangle$ is non-periodic, it cannot be expanded in the composite Hilbert space. To arrive at an expression that can be expanded in the composite Hilbert space, we use Eq. (29) to express the expectation value in terms of the periodic regular wave function,

$$
\left\langle\widetilde{0}\left|X_{i}\right| \widetilde{0}\right\rangle=\left\langle 0_{R}\left|\exp (i \mathcal{F}) X_{i} \exp (-i \mathcal{F})\right| 0_{R}\right\rangle=\left\langle 0_{R}\left|X_{i}\right| 0_{R}\right\rangle
$$

For the perturbations of the Hamiltonian in Eq. (3), the expectation value $\left\langle\widetilde{0}\left|X_{i}\right| \widetilde{0}\right\rangle$ may be experimentally determined for all perturbation strengths. All terms in the expansion of $\left\langle 0_{R}\left|X_{i}\right| 0_{R}\right\rangle$ in the perturbation strengths may therefore be obtained from experiments identifying at each order in the perturbation strength the molecular constants, i.e., molecular properties of the molecular system. The expansion of $\left\langle 0_{R}\left|X_{i}\right| 0_{R}\right\rangle$ in the perturbation strength may be expressed as

$$
\begin{aligned}
\left\langle 0_{R}\left|X_{i}\right| 0_{R}\right\rangle & =\left\langle\left\langle X_{i}\right\rangle\right\rangle_{0}+\sum_{j_{1}}\left\langle\left\langle X_{i} ; X_{j_{1}}\right\rangle\right\rangle_{\omega_{X_{j_{1}}}} \epsilon_{X_{j_{1}}}\left(\omega_{X_{j_{1}}}\right) \\
& \times \exp \left(-i \omega_{X_{j_{1}}} t\right)+\frac{1}{2} \sum_{j_{1}} \sum_{j_{2}}\left\langle\left\langle X_{i} ; X_{j_{1}}, X_{j_{2}}\right\rangle\right\rangle_{\omega_{X_{j_{1}}}, \omega_{X_{j_{2}}}} \\
& \times \epsilon_{X_{j_{1}}}\left(\omega_{X_{j_{1}}}\right) \epsilon_{X_{j_{2}}}\left(\omega_{X_{j_{2}}}\right) \exp \left[-i\left(\omega_{X_{j_{1}}}+\omega_{X_{j_{2}}}\right) t\right] \\
& +\cdots \\
& +\frac{1}{n !} \sum_{j_{1}} \cdots \sum_{j_{n}}\left\langle\left\langle X_{i} ; X_{j_{1}}, \ldots, X_{j_{n}}\right\rangle\right\rangle_{\omega_{X_{j_{1}}}, \ldots, \omega_{X_{j_{n}}}} \\
& \times \prod_{m=1}^{n} \epsilon_{X_{j_{m}}}\left(\omega_{X_{j_{m}}}\right) \exp \left(-i \sum_{m=1}^{n} \omega_{X_{j_{m}}} t\right),
\end{aligned}
$$

where the expansion coefficients are symmetric with respect to the operator-frequency pair permutations $\left(X_{j_{k}}, \omega_{X_{j_{k}}}\right)$ $\leftrightarrow\left(X_{j_{l}}, \omega_{X_{j_{l}}}\right)$, for $k, l=1,2, \ldots$ The zero-order term, $\left\langle\left\langle X_{i}\right\rangle\right\rangle_{0}$ $=\left\langle 0_{0}\left|X_{i}\right| 0_{0}\right\rangle$, is the static first-order molecular property. The expansion coefficient of the first-order term, $\left\langle\left\langle X_{i} ; X_{j_{1}}\right\rangle\right\rangle_{\omega_{X_{j_{1}}}}$, is the linear response function and measures how the molecular property described by the operator $X_{i}$ is altered linearly by applying a perturbation field $X_{j_{1}}$ oscillating with a frequency $\omega_{X_{j_{1}}} \cdot\left\langle\left\langle X_{i} ; X_{j_{1}}\right\rangle\right\rangle_{\omega_{X_{j_{1}}}}$ is a second-order molecular property. In general, the expansion coefficients, 
$\left\langle\left\langle X_{i} ; X_{j_{1}}, \ldots, X_{j_{n}}\right\rangle\right\rangle_{\omega_{X_{j_{1}}}, \ldots, \omega_{X_{j_{n}}}}$, are the $n$ th-order response functions and determine the response of the molecular property described by the operator $X_{i}$ to $n$ perturbations.

To see that the two definitions of molecular properties are identical, we first have to derive the generalized HellmannFeynman theorem for the quasi-energy. We therefore take the derivative of $\mathcal{E}$ in Eq. (55) with respect to a perturbation strength $\epsilon_{X_{i}}\left(\omega_{X_{i}}\right)$,

$$
\begin{aligned}
\frac{\mathrm{d} \mathcal{E}}{\mathrm{d} \epsilon_{X_{i}}\left(\omega_{X_{i}}\right)}= & \frac{\mathrm{d}\left\{\left\langle 0_{R}\left|H-i \frac{\partial}{\partial t}\right| 0_{R}\right\rangle\right\}_{T}}{\mathrm{~d} \epsilon_{X_{i}}\left(\omega_{X_{i}}\right)} \\
= & \left\{\left\langle 0_{R}\left|X_{i}\right| 0_{R}\right\rangle \exp \left(-i \omega_{X_{i}} t\right)\right\}_{T} \\
& +\left\{\left\langle\frac{\partial 0_{R}}{\partial \epsilon_{X_{i}}\left(\omega_{X_{i}}\right)}\left|H-i \frac{\partial}{\partial t}\right| 0_{R}\right\rangle\right.
\end{aligned}
$$

$$
\left.+\left\langle 0_{R}\left|H-i \frac{\partial}{\partial t}\right| \frac{\partial 0_{R}}{\partial \epsilon_{X_{i}}\left(\omega_{X_{i}}\right)}\right\rangle\right\}_{T},
$$

where we have used Eqs. (2) and (3). Using the variational principle in Eq. (58) and the fact that $\left|\frac{\partial 0_{R}}{\partial \epsilon_{X_{i}}}\right\rangle$ is an allowed variation, since it preserves periodicity of $\left|0_{R}\right\rangle$ and satisfies Eq. (61), we obtain from Eq. (165) the generalized HellmannFeynman theorem

$$
\frac{\mathrm{d} \mathcal{E}}{\mathrm{d} \epsilon_{X_{i}}\left(\omega_{X_{i}}\right)}=\left\{\left\langle 0_{R}\left|X_{i}\right| 0_{R}\right\rangle \exp \left(-i \omega_{X_{i}} t\right)\right\}_{T} .
$$

Inserting $\left\langle 0_{R}\left|X_{i}\right| 0_{R}\right\rangle$ of Eq. (164) into Eq. (166), we obtain

$$
\begin{aligned}
& \frac{\mathrm{d} \mathcal{E}}{\mathrm{d} \epsilon_{X_{i}}\left(\omega_{X_{i}}\right)}=\left\langle\left\langle X_{i}\right\rangle\right\rangle_{0}\left\{\exp \left(-i \omega_{X_{i}} t\right)\right\}_{T}+\sum_{j_{1}}\left\langle\left\langle X_{i} ; X_{j_{1}}\right\rangle\right\rangle_{\omega_{X_{j_{1}}}} \epsilon_{X_{j_{1}}}\left(\omega_{X_{j_{1}}}\right)\left\{\exp \left[-i\left(\omega_{X_{i}}+\omega_{X_{j_{1}}}\right) t\right]\right\}_{T} \\
& +\frac{1}{2} \sum_{j_{1}} \sum_{j_{2}}\left\langle\left\langle X_{i} ; X_{j_{1}}, X_{j_{2}}\right\rangle\right\rangle_{\omega_{X_{j_{1}}} \omega_{X_{j_{2}}}} \epsilon_{X_{j_{1}}}\left(\omega_{X_{j_{1}}}\right) \epsilon_{X_{j_{2}}}\left(\omega_{X_{j_{2}}}\right)\left\{\exp \left[-i\left(\omega_{X_{i}}+\omega_{X_{j_{1}}}+\omega_{X_{j_{2}}}\right) t\right]\right\}_{T} \\
& +\cdots+ \\
& \frac{1}{n !} \sum_{j_{1}} \ldots \sum_{j_{n}}\left\langle\left\langle X_{i} ; X_{j_{1}}, \ldots, X_{j_{n}}\right\rangle\right\rangle_{\omega_{X_{j_{1}}}, \ldots, \omega_{X_{j_{n}}}} \prod_{m=1}^{n} \epsilon_{X_{j_{m}}}\left(\omega_{X_{j_{m}}}\right)\left\{\exp \left[-i\left(\omega_{X_{i}}+\sum_{m=1}^{n} \omega_{X_{j_{m}}}\right) t\right]\right\}_{T} .
\end{aligned}
$$

Using Eq. (120) gives

$$
\begin{aligned}
\frac{\mathrm{d} \mathcal{E}}{\mathrm{d} \epsilon_{X_{i}}\left(\omega_{X_{i}}\right)}= & \left\langle\left\langle X_{i}\right\rangle\right\rangle_{0} \Delta\left(\omega_{X_{i}}\right)+\sum_{j_{1}}\left\langle\left\langle X_{i} ; X_{j_{1}}\right\rangle\right\rangle_{\omega_{X_{j_{1}}}} \epsilon_{X_{j_{1}}}\left(\omega_{X_{j_{1}}}\right) \Delta\left(\omega_{X_{i}}+\omega_{X_{j_{1}}}\right)+\frac{1}{2} \sum_{j_{1}} \sum_{j_{2}}\left\langle\left\langle X_{i} ; X_{j_{1}}, X_{j_{2}}\right\rangle\right\rangle_{\omega_{X_{j_{1}}} \omega_{X_{j_{2}}}} \\
& \times \epsilon_{X_{j_{1}}}\left(\omega_{X_{j_{1}}}\right) \epsilon_{X_{j_{2}}}\left(\omega_{X_{j_{2}}}\right) \Delta\left(\omega_{X_{i}}+\omega_{X_{j_{1}}}+\omega_{X_{j_{2}}}\right)+\cdots+\frac{1}{n !} \sum_{j_{1}} \cdots \sum_{j_{n}}\left\langle\left\langle X_{i} ; X_{j_{1}}, \ldots, X_{j_{n}}\right\rangle\right\rangle_{\omega_{X_{j_{1}}}, \ldots, \omega_{X_{j_{n}}}} \\
& \times \prod_{m=1}^{n} \epsilon_{X_{j_{m}}}\left(\omega_{X_{j_{m}}}\right) \Delta\left(\omega_{X_{i}}+\sum_{m=1}^{n} \omega_{X_{j m}}\right)
\end{aligned}
$$

Differentiating Eq. (168) with respect to $\epsilon_{X_{j_{1}}}\left(\omega_{X_{j_{1}}}\right) \ldots \epsilon_{X_{j_{n}}}\left(\omega_{X_{j_{n}}}\right)$ at the zero perturbation strength gives

$$
\begin{gathered}
\left(\frac{\mathrm{d}^{n+1} \mathcal{E}}{\mathrm{d} \epsilon_{X_{i}}\left(\omega_{X_{i}}\right) \mathrm{d} \epsilon_{X_{j_{1}}}\left(\omega_{X_{j_{1}}}\right) \cdots \mathrm{d} \epsilon_{X_{j_{n}}}\left(\omega_{X_{j_{n}}}\right)}\right)_{0} \\
=\left\langle\left\langle X_{i} ; X_{j_{1}}, \ldots, X_{j_{n}}\right\rangle\right\rangle_{\omega_{X_{j_{1}}}, \ldots, \omega_{X_{j_{n}}}} \\
\omega_{X_{i}}+\sum_{m=1}^{n} \omega_{X_{j_{m}}}=0,
\end{gathered}
$$

where we have used that response functions $\left\langle\left\langle X_{i} ; X_{j_{1}}, \ldots, X_{j_{n}}\right\rangle\right\rangle_{\omega_{X_{j_{1}}}, \ldots, \omega_{X_{j_{n}}}}$ are symmetric with respect to permutations of operator-frequency pairs $\left(X_{j_{k}}, \omega_{X_{j_{k}}}\right) \leftrightarrow$ $\left(X_{j_{l}}, \omega_{X_{j_{l}}}\right), \quad k, l=1,2, \ldots$ Using Eq. (65), we obtain from
Eq. (169)

$$
\begin{aligned}
& \left\langle\left\langle X_{i} ; X_{j_{1}}, \ldots, X_{j_{n}}\right\rangle\right\rangle_{\omega_{X_{j_{1}}}, \ldots, \omega_{X_{j_{n}}}} \\
& \quad=\mathcal{E}^{X_{i} X_{j_{1}} \cdots X_{j_{n}}}\left(\omega_{X_{i}}, \omega_{X_{j_{1}}}, \ldots, \omega_{X_{j_{n}}}\right), \quad \omega_{X_{i}}+\sum_{m=1}^{n} \omega_{X_{j_{m}}}=0
\end{aligned}
$$

and conclude that the traditional definitions of the response functions as the coefficients of the expansion in Eq. (164) are identical to the definitions from the quasi-energy expansion in Eq. (65).

When molecular properties are defined in terms of the expansion coefficients of the Taylor series in Eq. (164), here

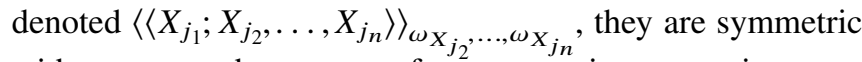
with respect to the operator-frequency pair permutations

$$
\left(X_{j_{k}}, \omega_{X_{j_{k}}}\right) \leftrightarrow\left(X_{j_{l}}, \omega_{X_{j_{l}}}\right), \quad k, l=2,3, \ldots
$$


Further, there is no constraint on the frequencies. The frequency constraint arises from the integration over time when the periodicity of $\left|0_{R}\right\rangle$ is introduced. In the above derivation, this is done when the expansion of $\left\langle 0_{R}\left|X_{i}\right| 0_{R}\right\rangle$ in Eq. (164) is inserted into the generalized HellmannFeynman theorem expression in Eq. (166) and the time averaging is carried out. Since from Eq. (170) the response functions are derivatives of the quasi-energy with respect to perturbation strengths, they are symmetric with respect to permutations of all the operator-frequency pairs, including the first operator-frequency pair $\left(X_{j_{1}}, \omega_{X_{j_{1}}}\right)$,

$$
\left(X_{j_{k}}, \omega_{X_{j_{k}}}\right) \leftrightarrow\left(X_{j_{l}}, \omega_{X_{j_{l}}}\right), \quad k, l=1,2, \ldots
$$

The extension of the symmetry relation to include also the first operator-frequency pair $\left(X_{j_{1}}, \omega_{X_{j_{1}}}\right)$ is far from trivial to prove when molecular properties are defined through the expansion in Eq. (164). ${ }^{12}$

For a variational wave function, the generalized HellmannFeynman theorem is satisfied and molecular properties are identical whether they are defined in terms of derivatives of the quasi-energy or from an average value of a perturbation operator. However, for a non-variational wave function, where the generalized Hellmann-Feynman theorem is not satisfied, molecular properties are expressed in terms of quasi-energy derivatives. The obtained expressions then deviate from the simple average value expression due to the non-variational nature of the wave function. From a theoretical point of view, the quasi-energy expressed as an eigenvalue of the Hermitian eigenvalue equation is the fundamental quantity for a molecular system when a periodic perturbation is imposed, as the energy is the fundamental quantity when the perturbation is static. This should be reflected in the way molecular response properties are defined.

\section{RESPONSE FUNCTIONS FROM THE HERMITIAN EIGENVALUE EQUATION SOLVED VIA PROJECTION}

In this section, we consider how response functions may be obtained when the Hermitian eigenvalue equation [Eq. (32)] is solved via projection in the composite Hilbert space. The development we present allows for determination of response functions where the parametrization of the ground state for the unperturbed system differs from the parametrization of its time evolution. This allows response functions to be determined for a new general class of approximate wave function models, extending the range of wave function models for which response functions can be determined.

In Sec. IV A, we parametrize the regular wave function of Eq. (68) where the parametrization of the unperturbed ground state may be different from the parametrization of the time evolution. In Sec. IV B, we describe how the Hermitian eigenvalue equation [Eq. (32)] may be solved via projection. In Secs. IV C and IV D, we introduce the quasi-energy Lagrangian for the solution of Eq. (32), and in Sec. IV E, response functions are derived from the quasienergy Lagrangian.

\section{A. Parametrization of the regular wave function}

In this section, we consider wave function models where projection is used to solve the Hermitian eigenvalue equation. In Subsection IV A 1, we consider the parametrization of the ground state for the unperturbed system, whereas in Subsection IV A 2, the parametrization of the time evolution of this state is described.

\section{Parametrization of the unperturbed state}

The eigenstate $\left|0_{0}\right\rangle$ of the unperturbed Hamiltonian $H_{0}$ may be parametrized in terms of an orthonormal basis consisting of a reference state $|\mathscr{R}\rangle$ and its orthogonal complement $\{|k\rangle\}$,

$$
|\mathcal{B}\rangle=\{|\mathscr{R}\rangle,|k\rangle\} .
$$

With this parametrization, $\left|0_{0}\right\rangle$ may be written as

$$
\left|0_{0}\right\rangle=N_{0}\left|0_{I_{0}}\right\rangle
$$

where

$$
\left|0_{I_{0}}\right\rangle=\exp \left(B_{0}\right)|\Re\rangle
$$

and $N_{0}$ is the normalization constant,

$$
N_{0}=\left\langle\mathscr{R}\left|\exp \left(B_{0}\right)^{\dagger} \exp \left(B_{0}\right)\right| \mathscr{R}\right\rangle^{-1 / 2} .
$$

We discuss below the parametrization of the exponential operator $\exp \left(B_{0}\right)$. To comprehend coupled cluster theory, we consider $\left|0_{I_{0}}\right\rangle$ states that are intermediate normalized,

$$
\left\langle\mathscr{R} \mid 0_{I_{0}}\right\rangle=1,
$$

hence the subscript $I$ in $\left|0_{I_{0}}\right\rangle$. The intermediate normalization condition may be satisfied by requiring that the action of $B_{0}$ on any state $|P\rangle$ leads to a state that is orthogonal to the reference state $|\mathscr{R}\rangle$,

$$
B_{0}|P\rangle=\sum_{k} b_{k}^{(0)}|k\rangle
$$

We express the operators $B_{0}$ satisfying Eq. (178) in the generic form

$$
B_{0}=\sum_{k} b_{k}^{(0)} \beta_{k}
$$

The operator $\beta_{k}$ may take on the form of a state-transfer operator

$$
\beta_{k}=|k\rangle\langle\mathscr{R}|
$$

and Eq. (175) then describes a linear parametrization of $\left|0_{I_{0}}\right\rangle$ in the basis $|\mathcal{B}\rangle$ of Eq. (173),

$$
\left|0_{I_{0}}\right\rangle=\exp \left(B_{0}\right)|\mathscr{R}\rangle=|\mathscr{R}\rangle+\sum_{k} b_{k}^{(0)}|k\rangle .
$$

The advantage of expressing a linear parametrization of a wave function in terms of an exponential operator is that the inverse of the wave function may then straightforwardly be determined,

$$
\left\langle 0_{I_{0}} \mid 0_{I_{0}}\right\rangle=\left\langle\mathscr{R}\left|\exp \left(-B_{0}\right) \exp \left(B_{0}\right)\right| \mathscr{R}\right\rangle=1 .
$$

The operator $\beta_{k}$ may alternatively be defined as a manybody orbital excitation operator carrying out excitations from 
orbitals occupied in the reference state to its orthogonal complement

$$
\beta_{k}=\tau_{k}
$$

Equation (175) then describes an exponential parametrization of $\left|0_{I_{0}}\right\rangle$, as in coupled cluster theory. Using the exponential operator form of a linear parametrization allows us to develop a generic expression for the quasi-energy Lagrangian that encompasses both a linear and exponential parametrization of $\left|0_{I_{0}}\right\rangle$.

For a given choice of the operator manifold, the generic excitation operators commute

$$
\left[\beta_{k}, \beta_{j}\right]=0
$$

The operators $\beta_{k}$ satisfy

$$
\beta_{k}|\mathscr{R}\rangle=|k\rangle
$$

as follows from Eq. (178). Using Eq. (178) and the orthonormality of the basis in Eq. (173), we have for any state $|P\rangle$

$$
\left\langle\mathscr{R}\left|\exp \left(-B_{0}\right)\right| P\right\rangle=\left\langle\mathscr{R}\left|\left(1-B_{0}+\frac{1}{2} B_{0}^{2}+\cdots\right)\right| P\right\rangle=\langle\mathscr{R} \mid P\rangle .
$$

Since Eq. (186) is fulfilled for any state $|P\rangle$, it follows that

$$
\langle\mathscr{R}| B_{0}=0
$$

and

$$
\langle\mathscr{R}|=\langle\mathscr{R}| \exp \left(-B_{0}\right)
$$

Inserting Eq. (174) in Eq. (87b) and using Eq. (175), we obtain

$$
H_{0} \exp \left(B_{0}\right)|\mathscr{R}\rangle=E_{0} \exp \left(B_{0}\right)|\mathscr{R}\rangle
$$

The reference to the normalization constant thus vanishes trivially, when the Schrödinger equation is solved for a timeindependent unperturbed system. Multiplying Eq. (189) by $\exp \left(-B_{0}\right)$, we obtain the similarity-transformed Schrödinger equation

$$
\exp \left(-B_{0}\right) H_{0} \exp \left(B_{0}\right)|\mathscr{R}\rangle=E_{0}|\mathscr{R}\rangle
$$

$E_{0}$ is thus the eigenvalue of a similarity-transformed Hamiltonian

$$
H_{0}^{B_{0}}=\exp \left(-B_{0}\right) H_{0} \exp \left(B_{0}\right)
$$

Equation (190) emphasizes that the eigenvalues of the Schrödinger equation do not change when the Hamiltonian is subjected to a similarity transformation. Equation (190) may be solved by projecting it against the basis

$$
\langle\mathcal{B}|=\{\langle\mathscr{R}|,\langle k|\},
$$

giving

$$
\begin{aligned}
\left\langle\mathscr{R}\left|\exp \left(-B_{0}\right) H_{0} \exp \left(B_{0}\right)\right| \mathscr{R}\right\rangle & =E_{0}, \\
\left\langle k\left|\exp \left(-B_{0}\right) H_{0} \exp \left(B_{0}\right)\right| \mathscr{R}\right\rangle & =0 .
\end{aligned}
$$

For the determination of response functions, it turns out to be convenient to express the solution to the Schrödinger equation for the unperturbed system in Eqs. (193a) and (193b) in terms of a Lagrangian, where Eq. (193b) is added as a constraint to Eq. (193a) using undetermined Lagrangian multipliers, $\bar{b}_{k}^{(0)}$,

$$
L_{0}=\left\langle\mathscr{R}\left|H_{0}^{B_{0}}\right| \mathscr{R}\right\rangle+\sum_{k} \bar{b}_{k}^{(0)}\left\langle k\left|H_{0}^{B_{0}}\right| \mathscr{R}\right\rangle,
$$

where we have used Eq. (191). The Lagrangian, $L_{0}$, in Eq. (194) is stationary with respect to the Lagrangian multipliers

$$
\frac{\partial L_{0}}{\partial \bar{b}_{k}^{(0)}}=\left\langle k\left|H_{0}^{B_{0}}\right| \mathscr{R}\right\rangle=0 .
$$

The multipliers, $\bar{b}_{k}^{(0)}$, are undetermined and may be chosen such that the Lagrangian in Eq. (194) is also stationary with respect to the coefficients $b_{k}^{(0)}$ in Eq. (179),

$$
\begin{aligned}
\frac{\partial L_{0}}{\partial b_{k}^{(0)}}= & \left\langle\mathscr{R}\left|\left[H_{0}^{B_{0}}, \beta_{k}\right]\right| \mathscr{R}\right\rangle \\
& +\sum_{m} \bar{b}_{m}^{(0)}\left\langle m\left|\left[H_{0}^{B_{0}}, \beta_{k}\right]\right| \mathscr{R}\right\rangle=0 .
\end{aligned}
$$

\section{Parametrization of the time evolution}

The formal separation of the description of the unperturbed ground state and the time evolution of this state may be achieved by parametrizing the unit normalized regular wave function $\left|0_{R}(t, \epsilon)\right\rangle$ of Eq. (68) as

$$
\left|0_{R}(t, \epsilon)\right\rangle=N(t, \epsilon)\left|0_{I}(t, \epsilon)\right\rangle,
$$

where

$$
\left|0_{I}(t, \epsilon)\right\rangle=\exp \left(B_{0}\right) \exp (B(t))|\mathscr{R}\rangle
$$

and $N(t, \epsilon)$ is the time-dependent normalization constant

$N(t, \epsilon)=\left\langle\mathscr{R}\left|\exp (B(t))^{\dagger} \exp \left(B_{0}\right)^{\dagger} \exp \left(B_{0}\right) \exp (B(t))\right| \mathscr{R}\right\rangle^{-1 / 2}$.

The operator $B_{0}$ parametrizes the unperturbed ground state (as discussed in Sec. IV A 1) and the operator $B(t)$ parametrizes the perturbation-induced time evolution of this state. Similarly to $B_{0}$, the operator $B(t)$ is defined such that $\left|0_{I}(t, \epsilon)\right\rangle$ is intermediate normalized against $\langle\mathscr{R}|$,

$$
\left\langle\mathscr{R} \mid 0_{I}(t, \epsilon)\right\rangle=1,
$$

and therefore the action of $B(t)$ on any state leads to a state that is orthogonal to the reference state $|\mathscr{R}\rangle$. Analogous to Eq. (179), $B(t)$ may be written as

$$
B(t)=\sum_{k} b_{k}(t) \beta_{k}
$$

A time-dependent analogue of Eq. (188) also holds for $B(t)$,

$$
\langle\mathscr{R}|=\langle\mathscr{R}| \exp (-B(t)) \text {. }
$$


The operators $B_{0}$ and $B(t)$ in general do not commute, for example, if $B_{0}$ is expressed in terms of the many-body operators in Eq. (183), while $B(t)$ is expressed in terms of the state-transfer operators in Eq. (180). The order that is chosen in Eq. (198) allows to describe the time evolution in terms of a similarity-transformed Hamiltonian $H^{B_{0}}$ containing the time-independent operator $\exp \left(B_{0}\right)$.

Since the normalization constant $N(t, \epsilon)$ in Eq. (199) is time-dependent, it is not trivially eliminated when $\left|0_{R}(t, \epsilon)\right\rangle$ of Eq. (197) is inserted in the Hermitian eigenvalue equation [Eq. (32)] in contrast to the time-independent case where the normalization constant can be removed straightforwardly giving Eq. (189). In Sec. IV B, we show how the reference to the normalization constant can be eliminated when the Hermitian eigenvalue equation in Eq. (32) is solved via projection in the composite Hilbert space and that the solution to the Hermitian eigenvalue equation then may be expressed as a straightforward generalization of Eqs. (193a) and (193b).

\section{B. Projected Hermitian eigenvalue equation}

We now describe how the solution to the time-dependent Schrödinger equation [Eq. (32)] may be determined using projection. Inserting Eq. (197) in the Hermitian eigenvalue equation [Eq. (32)] and using Eq. (198) gives

$$
\begin{aligned}
(H- & \left.i \frac{\partial}{\partial t}-\dot{\mathcal{F}}_{P_{0}}-i N^{-1} \dot{N}\right) \exp \left(B_{0}\right) \exp (B(t))|\mathscr{R}\rangle \\
& =\mathcal{E} \exp \left(B_{0}\right) \exp (B(t))|\mathscr{R}\rangle .
\end{aligned}
$$

Multiplying Eq. (203) by $\exp (-B(t)) \exp \left(-B_{0}\right)$, we obtain

$$
\begin{aligned}
\exp ( & -B(t))\left(H^{B_{0}}-i \frac{\partial}{\partial t}\right) \exp (B(t))|\Re\rangle \\
= & \left(\mathcal{E}+\dot{\mathcal{F}}_{P_{0}}+i \frac{\mathrm{d}}{\mathrm{d} t} \ln N\right)|\mathscr{R}\rangle,
\end{aligned}
$$

where we have used that $B_{0}$ is time-independent and where the similarity transformed Hamiltonian $H^{B_{0}}$ is defined analogously to $H_{0}^{B_{0}}$ of Eq. (191). Projecting Eq. (204) against the basis $\langle\mathcal{B}|$ of Eq. (192), we obtain the set of equations

$$
\begin{gathered}
\operatorname{Re}\left\langle\mathscr{R}\left|\exp (-B(t))\left(H^{B_{0}}-i \frac{\partial}{\partial t}\right) \exp (B(t))\right| \mathscr{R}\right\rangle=\mathcal{E}+\dot{\mathcal{F}}_{P_{0}} \\
\operatorname{Im}\left\langle\mathscr{R}\left|\exp (-B(t))\left(H^{B_{0}}-i \frac{\partial}{\partial t}\right) \exp (B(t))\right| \mathscr{R}\right\rangle=\frac{\mathrm{d}}{\mathrm{d} t} \ln N \\
\left\langle k\left|\exp (-B(t))\left(H^{B_{0}}-i \frac{\partial}{\partial t}\right) \exp (B(t))\right| \mathscr{R}\right\rangle=0
\end{gathered}
$$

where we have used that $\mathcal{E}$ and $\dot{\mathcal{F}}_{P_{0}}$ are real and $i \frac{\mathrm{d}}{\mathrm{d} t} \ln N$ is purely imaginary (since $N$ is real by construction-see Eq. (199)). Time-averaging the set of equations (205) and using Eqs. (41) and (93), we obtain

$$
\begin{aligned}
& \mathcal{E}=\operatorname{Re}\left\{\left\langle\mathscr{R}\left|\exp (-B(t))\left(H^{B_{0}}-i \frac{\partial}{\partial t}\right) \exp (B(t))\right| \mathscr{R}\right\rangle\right\}_{T}, \\
& 0=\operatorname{Im}\left\{\left\langle\mathscr{R}\left|\exp (-B(t))\left(H^{B_{0}}-i \frac{\partial}{\partial t}\right) \exp (B(t))\right| \mathscr{R}\right\rangle\right\}_{T},
\end{aligned}
$$

$\left\{\left\langle k\left|\exp (-B(t))\left(H^{B_{0}}-i \frac{\partial}{\partial t}\right) \exp (B(t))\right| \mathscr{R}\right\rangle\right\}_{T}=0$.

The explicit reference to the normalization constant therefore vanishes when the Hermitian eigenvalue equation [Eq. (32)] is solved via projection in the composite Hilbert space. Equation (206) shows that the solution to the Hermitian eigenvalue equation [Eq. (32)] is obtained by first solving Eq. (193b) to obtain $H^{B_{0}}$, then solving Eq. (206c) and inserting this solution to Eq. (206a) yielding $\mathcal{E}$. Knowledge of the time-periodic phase $\mathcal{F}_{P_{0}}$ is thus not required for determining $\left|0_{R}\right\rangle$ and $\mathcal{E}$.

The set of equations (206) may alternatively be obtained in a single step by projecting Eq. (204) directly in the composite Hilbert space and using the definition of the inner product in this space [Eq. (36)]. The right-hand side of Eq. (206a) would then not be ensured to be real. This does not pose a problem for an exact representation of the regular wave function $\left|0_{R}(t, \epsilon)\right\rangle$, where the quasi-energy $\mathcal{E}$ is real being an eigenvalue of the Hermitian operator. For approximate wave function models, the time averages of $\frac{\mathrm{d}}{\mathrm{d} t} \ln N$ and $\dot{\mathcal{F}}_{P_{0}}$ still vanish. However, $\left\langle\mathscr{R}\left|\left(H^{B_{0}}-i \frac{\partial}{\partial t}\right) \exp (B(t))\right| \mathscr{R}\right\rangle$ may be complex. Using the two-step approach above to derive the set of equations (206) ensures that the quasi-energy, and hence also molecular properties, is real also for approximate wave function models.

\section{Stationary conditions for the quasi-energy Lagrangian}

Molecular response properties may be determined by solving Eq. (206c) explicitly and then determining $\mathcal{E}$ from Eq. (206a). However, to get variational flexibility to simplify the determination of response functions, we introduce, as for the static case, a Lagrangian, where Eq. (206c) is added as a constraint to Eq. (206a) using frequency-dependent undetermined Lagrangian multipliers, $\bar{b}_{k}\left(\omega_{K}\right)$, which are defined analogously to $b_{k}\left(\omega_{K}\right)$ of Eq. (114),

$$
\bar{b}_{k}(t)=\sum_{K} \bar{b}_{k}\left(\omega_{K}\right) \exp \left(-i \omega_{K} t\right) .
$$

The Lagrangian then becomes

$$
\begin{aligned}
\mathcal{L}= & \operatorname{Re}\left\{\left\langle\mathscr{R}\left|\exp (-B(t))\left(H^{B_{0}}-i \frac{\partial}{\partial t}\right) \exp (B(t))\right| \mathscr{R}\right\rangle\right\}_{T} \\
& +\sum_{k K} \bar{b}_{k}\left(\omega_{K}\right)\left\{\langle k| \exp (-B(t))\left(H^{B_{0}}-i \frac{\partial}{\partial t}\right)\right. \\
& \left.\times \exp (B(t))|\mathscr{R}\rangle \exp \left(-i \omega_{K} t\right)\right\}_{T} .
\end{aligned}
$$

The Lagrangian multipliers, $\bar{b}_{k}\left(\omega_{K}\right)$, contain the zero-order contribution, $\bar{b}_{k}^{(0)}$. In the limit of a time-independent unperturbed system, the Lagrangian $\mathcal{L}$ therefore reduces to $L_{0}$ [Eq. (194)]. 
The Lagrangian of Eq. (208) is per construction stationary with respect to the multipliers

$$
\begin{aligned}
\frac{\partial \mathcal{L}}{\partial \bar{b}_{k}\left(\omega_{K}\right)}= & \left\{\langle k| \exp (-B(t))\left(H^{B_{0}}-i \frac{\partial}{\partial t}\right)\right. \\
& \left.\times \exp (B(t))|\mathscr{R}\rangle \exp \left(-i \omega_{K} t\right)\right\}_{T}=0 .
\end{aligned}
$$

The Lagrangian multipliers are undetermined and are chosen such that the Lagrangian of Eq. (208) is stationary also with respect to the coefficients $b_{k}\left(\omega_{K}\right)$ (entering $B(t)$ of Eq. (201) in the frequency domain),

$$
\frac{\partial \mathcal{L}}{\partial b_{k}\left(\omega_{K}\right)}=0 .
$$

We now consider a Lagrangian, where the real part is taken on both terms in Eq. (208),

$$
\begin{aligned}
\mathcal{L}= & \operatorname{Re}\left(\left\{\left\langle\mathscr{R}\left|\exp (-B(t))\left(H^{B_{0}}-i \frac{\partial}{\partial t}\right) \exp (B(t))\right| \mathscr{R}\right\rangle\right\}_{T}\right. \\
& +\sum_{k K} \bar{b}_{k}\left(\omega_{K}\right)\left\{\left\langle k\left|\exp (-B(t))\left(H^{B_{0}}-i \frac{\partial}{\partial t}\right) \exp (B(t))\right| \mathscr{R}\right\rangle\right. \\
& \left.\left.\times \exp \left(-i \omega_{K} t\right)\right\}_{T}\right) .
\end{aligned}
$$

To show that the two forms of the Lagrangian, Eqs. (208) and (211), lead to the same stationary points, we consider $\bar{b}_{k}\left(\omega_{K}\right)$ and $\bar{b}_{k}^{*}\left(\omega_{K}\right)$ as independent parameters. Then, noting that the first term in Eq. (211) does not depend on multipliers, we obtain

$$
\begin{aligned}
\frac{\partial \mathcal{L}}{\partial \bar{b}_{m}\left(\omega_{M}\right)}= & \frac{1}{2} \frac{\partial}{\partial \bar{b}_{m}\left(\omega_{M}\right)}\left(\sum_{k K} \bar{b}_{k}\left(\omega_{K}\right)\left\{\left\langle k\left|\exp (-B(t))\left(H^{B_{0}}-i \frac{\partial}{\partial t}\right) \exp (B(t))\right| \mathscr{R}\right\rangle \exp \left(-i \omega_{K} t\right)\right\}_{T}\right. \\
& \left.+\sum_{k K} \bar{b}_{k}^{*}\left(\omega_{K}\right)\left\{\left\langle k\left|\exp (-B(t))\left(H^{B_{0}}-i \frac{\partial}{\partial t}\right) \exp (B(t))\right| \mathscr{R}\right\rangle^{*} \exp \left(i \omega_{K} t\right)\right\}_{T}\right) \\
= & \frac{1}{2}\left\{\left\langle m\left|\exp (-B(t))\left(H^{B_{0}}-i \frac{\partial}{\partial t}\right) \exp (B(t))\right| \mathscr{R}\right\rangle \exp \left(-i \omega_{M} t\right)\right\}_{T} .
\end{aligned}
$$

A similar equation also holds for $\bar{b}_{m}^{*}\left(\omega_{M}\right)$. The Lagrangian of Eq. (211) is thus stationary with respect to variations in $\bar{b}_{m}\left(\omega_{M}\right)$ and $\bar{b}_{m}^{*}\left(\omega_{M}\right)$ if and only if Eq. (206c) is satisfied. The Lagrangian expression in Eq. (208) and in Eq. (211) thus both have stationary points at the solution to the projected Schrödinger equation [Eq. (206c)]. Further, it follows from Eqs. (208) and (211) that the two forms of the Lagrangian are equal at the stationary points.

The set of equations (206) implies that at the stationary points the Lagrangian of Eq. (211) is equal to the quasi-energy of Eq. (55),

$\mathcal{L}=\mathcal{E} \quad$ for $\quad \delta \mathcal{E}=0, \frac{\partial \mathcal{L}}{\partial \bar{b}_{k}\left(\omega_{K}\right)}=0, \frac{\partial \mathcal{L}}{\partial b_{k}\left(\omega_{K}\right)}=0$.

We therefore denote the Lagrangian $\mathcal{L}$ of Eq. (211) as the quasi-energy Lagrangian. It follows from Eq. (213) that at the stationary points the derivatives of $\mathcal{E}$ and $\mathcal{L}$ with respect to the perturbation strength are equal, in particular

$$
\begin{aligned}
\frac{\mathrm{d} \mathcal{E}}{\mathrm{d} \epsilon_{X_{i}}\left(\omega_{X_{i}}\right)} & =\frac{\mathrm{d} \mathcal{L}}{\mathrm{d} \epsilon_{X_{i}}\left(\omega_{X_{i}}\right)} \\
\text { for } \delta \mathcal{E} & =0, \frac{\partial \mathcal{L}}{\partial \bar{b}_{k}\left(\omega_{K}\right)}=0, \frac{\partial \mathcal{L}}{\partial b_{k}\left(\omega_{K}\right)}=0 .
\end{aligned}
$$

When the Hermitian eigenvalue equation is solved via projection, molecular response properties may therefore be obtained as derivatives of the real quasi-energy Lagrangian $\mathcal{L}$ of Eq. (211), in the same way as molecular response properties are obtained as derivatives of the real quasi-energy $\mathcal{E}$ of
Eq. (55), when the Hermitian eigenvalue equation is solved using the variational principle. For exact wave functions, the two approaches lead to the same molecular response properties.

\section{Complex quasi-energy Lagrangian and its stationary conditions}

In Subsection IV D 1, we derive an explicit expression for the complex quasi-energy Lagrangian, and in Subsection IV D 2, the stationary condition is derived for this Lagrangian.

\section{Complex quasi-energy Lagrangian}

To determine molecular properties, it is convenient to express the real quasi-energy Lagrangian, $\mathcal{L}$ of Eq. (211), in terms of the complex quasi-energy Lagrangian, ${ }^{c} \mathcal{L}$,

$$
\mathcal{L}=\operatorname{Re}{ }^{c} \mathcal{L}=\frac{1}{2}\left({ }^{c} \mathcal{L}+{ }^{c} \mathcal{L}^{*}\right),
$$

where

$$
\begin{aligned}
c^{c} \mathcal{L}= & \left\{\left\langle\mathscr{R}\left|\exp (-B(t))\left(H^{B_{0}}-i \frac{\partial}{\partial t}\right) \exp (B(t))\right| \mathscr{R}\right\rangle\right\}_{T} \\
& +\sum_{k K} \bar{b}_{k}\left(\omega_{K}\right)\left\{\langle k| \exp (-B(t))\left(H^{B_{0}}-i \frac{\partial}{\partial t}\right)\right. \\
& \left.\times \exp (B(t))|\mathscr{R}\rangle \exp \left(-i \omega_{K} t\right)\right\}_{T} .
\end{aligned}
$$


Further, it is convenient to split the complex quasi-energy Lagrangian, ${ }^{c} \mathcal{L}$ of Eq. (216), into the contributions similar to the ones obtained for the quasi-energy in Eq. (133). Using Eqs. (2) and (3), we may write the complex quasi-energy Lagrangian as

$$
\begin{aligned}
c_{\mathcal{L}}= & \left\{\left\langle\mathscr{R}\left|\exp (-B(t)) H_{0}^{B_{0}} \exp (B(t))\right| \mathscr{R}\right\rangle\right\}_{T} \\
& +\sum_{k K} \bar{b}_{k}\left(\omega_{K}\right)\left\{\left\langle k\left|\exp (-B(t))\left(H_{0}^{B_{0}}-i \frac{\partial}{\partial t}\right) \exp (B(t))\right| \mathscr{R}\right\rangle \exp \left(-i \omega_{K} t\right)\right\}_{T} \\
& +\sum_{j_{1}}\left\{\left\langle\mathscr{R}\left|\exp (-B(t)) X_{j_{1}}^{B_{0}} \exp (B(t))\right| \mathscr{R}\right\rangle \exp \left(-i \omega_{X_{j_{1}}} t\right)\right\}_{T} \epsilon_{X_{j_{1}}}\left(\omega_{X_{j_{1}}}\right) \\
& +\sum_{j_{1}} \sum_{k K} \bar{b}_{k}\left(\omega_{K}\right)\left\{\left\langle k\left|\exp (-B(t)) X_{j_{1}}^{B_{0}} \exp (B(t))\right| \mathscr{R}\right\rangle \exp \left(-i\left(\omega_{K}+\omega_{X_{j_{1}}}\right) t\right)\right\}_{T} \epsilon_{X_{j_{1}}}\left(\omega_{X_{j_{1}}}\right),
\end{aligned}
$$

where we have used that

$$
\begin{aligned}
\left\langle\mathscr{R}\left|\exp (-B(t)) \frac{\partial}{\partial t} \exp (B(t))\right| \mathscr{R}\right\rangle & =\left\langle\mathscr{R}\left|\frac{\partial}{\partial t} \exp (B(t))\right| \mathscr{R}\right\rangle \\
& =\frac{\mathrm{d}}{\mathrm{d} t}\langle\mathscr{R}|\exp (B(t))| \mathscr{R}\rangle \\
& =\frac{\mathrm{d}}{\mathrm{d} t}\langle\mathscr{R} \mid \mathscr{R}\rangle=0, \quad(21
\end{aligned}
$$

as follows from Eq. (202) and the time independence of $|\mathscr{R}\rangle$. The complex quasi-energy Lagrangian ${ }^{c} \mathcal{L}$ in Eq. (217) may be written in the form

$$
{ }^{c} \mathcal{L}={ }^{c} \mathcal{L}_{H_{0}}+{ }^{c} \mathcal{L}_{F}+\sum_{j_{1}}{ }^{c} \mathcal{L}_{X_{j_{1}}} \epsilon_{X_{j_{1}}}\left(\omega_{X_{j_{1}}}\right),
$$

where

$$
\begin{aligned}
{ }^{c} \mathcal{L}_{H_{0}}= & \left\{\left\langle\mathscr{R}\left|\exp (-B(t)) H_{0}^{B_{0}} \exp (B(t))\right| \mathscr{R}\right\rangle\right\}_{T} \\
& +\sum_{k K} \bar{b}_{k}\left(\omega_{K}\right)\left\{\left\langle k\left|\exp (-B(t)) H_{0}^{B_{0}} \exp (B(t))\right| \mathscr{R}\right\rangle\right. \\
& \left.\times \exp \left(-i \omega_{K} t\right)\right\}_{T}, \\
{ }^{c} \mathcal{L}_{F}= & -i \sum_{k K} \bar{b}_{k}\left(\omega_{K}\right)\left\{\left\langle k\left|\exp (-B(t)) \frac{\partial}{\partial t} \exp (B(t))\right| \mathscr{R}\right\rangle\right. \\
& \left.\times \exp \left(-i \omega_{K} t\right)\right\}_{T} \\
= & -i \sum_{k m K} \bar{b}_{k}\left(\omega_{K}\right)\left\{\dot{b}_{m}(t) \exp \left(-i \omega_{K} t\right)\right\}_{T}\left\langle k\left|\beta_{m}\right| \mathscr{R}\right\rangle,
\end{aligned}
$$

and

$$
\begin{aligned}
{ }^{c} \mathcal{L}_{X_{j_{1}}}= & \left\{\left\langle\mathscr{R}\left|\exp (-B(t)) X_{j_{1}}^{B_{0}} \exp (B(t))\right| \mathscr{R}\right\rangle \exp \left(-i \omega_{X_{j_{1}}} t\right)\right\}_{T} \\
& +\sum_{k K} \bar{b}_{k}\left(\omega_{K}\right)\left\{\left\langle k\left|\exp (-B(t)) X_{j_{1}}^{B_{0}} \exp (B(t))\right| \mathscr{R}\right\rangle\right. \\
& \left.\times \exp \left(-i\left(\omega_{K}+\omega_{X_{j_{1}}}\right) t\right)\right\}_{T} .
\end{aligned}
$$

To obtain the second equality in Eq. (221), we have used Eq. (201).

\section{Stationary conditions for the complex quasi-energy Lagrangian}

We have shown that the real quasi-energy Lagrangian $\mathcal{L}$ of Eq. (211) is stationary with respect to the wave-function coefficients $b_{k}\left(\omega_{K}\right)$ and Lagrangian multipliers $\bar{b}_{k}\left(\omega_{K}\right)$ and will now show that this implies that the complex quasi-energy Lagrangian ${ }^{c} \mathcal{L}$ is also stationary with respect to $b_{k}\left(\omega_{K}\right)$ and $\bar{b}_{k}\left(\omega_{K}\right)$. To do this, we first note from Eqs. (219)-(222) that ${ }^{c} \mathcal{L}$ depends on a set of wave-function coefficients, $\mathbf{b}\left(\omega_{K}\right)$, entering $B(t)$ of Eq. (201) in the frequency domain, and a set of Lagrangian multipliers, $\overline{\mathbf{b}}\left(\omega_{K}\right)$, in Eq. (207),

$$
{ }^{c} \mathcal{L}={ }^{c} \mathcal{L}\left(\mathbf{b}\left(\omega_{K}\right), \overline{\mathbf{b}}\left(\omega_{K}\right)\right) .
$$

Similarly, ${ }^{c} \mathcal{L}^{*}$ depends on $\mathbf{b}^{*}\left(\omega_{K}\right)$ and $\overline{\mathbf{b}}^{*}\left(\omega_{K}\right)$,

$$
{ }^{c} \mathcal{L}^{*}={ }^{c} \mathcal{L}^{*}\left(\mathbf{b}^{*}\left(\omega_{K}\right), \overline{\mathbf{b}}^{*}\left(\omega_{K}\right)\right) .
$$

Using that ${ }^{c} \mathcal{L}^{*}$ does not depend on $\mathbf{b}\left(\omega_{K}\right)$ and $\overline{\mathbf{b}}\left(\omega_{K}\right)$ and the stationarity of $\mathcal{L}$, we obtain that the complex quasi-energy Lagrangian, ${ }^{c} \mathcal{L}$ of Eq. (219), is stationary with respect to $b_{k}\left(\omega_{K}\right)$ and $\bar{b}_{k}\left(\omega_{K}\right)$,

$$
\begin{gathered}
\frac{\partial^{c} \mathcal{L}}{\partial b_{k}\left(\omega_{K}\right)}=\frac{\partial}{\partial b_{k}\left(\omega_{K}\right)}\left(2 \mathcal{L}-{ }^{c} \mathcal{L}^{*}\right)=2 \frac{\partial \mathcal{L}}{\partial b_{k}\left(\omega_{K}\right)}=0, \\
\frac{\partial^{c} \mathcal{L}}{\partial \bar{b}_{k}\left(\omega_{K}\right)}=\frac{\partial}{\partial \bar{b}_{k}\left(\omega_{K}\right)}\left(2 \mathcal{L}-{ }^{c} \mathcal{L}^{*}\right)=2 \frac{\partial \mathcal{L}}{\partial \bar{b}_{k}\left(\omega_{K}\right)}=0 .
\end{gathered}
$$

Similarly, ${ }^{c} \mathcal{L}^{*}$ is stationary with respect to $b_{k}^{*}\left(\omega_{K}\right)$ and $\bar{b}_{k}^{*}\left(\omega_{K}\right)$.

\section{E. Molecular properties from the quasi-energy Lagrangian}

In Subsection IV E 1, the quasi-energy Lagrangian is expanded in orders of the perturbation strength, and in Subsection IV E 2, stationary conditions are established for each term in the order expansion.

\section{Molecular properties from an order expansion of the quasi-energy Lagrangian}

Following the developments in Sec. III B, we expand the quasi-energy Lagrangian as in Eqs. (134) and (135),

$$
\mathcal{L}=\sum_{n=0}^{\infty} \mathcal{L}^{(n)}
$$


where

$$
\begin{aligned}
\mathcal{L}^{(n)}= & \frac{1}{n !} \sum_{j_{1} \leq \cdots \leq j_{n}} \mathcal{N}_{\omega_{X_{j_{1}}} \ldots \omega_{X_{j n}}}^{X_{j_{1} \ldots X_{j_{n}}}} \mathcal{L}^{X_{j_{1}} \ldots X_{j_{n}}}\left(\omega_{X_{j_{1}}}, \ldots, \omega_{X_{j_{n}}}\right) \\
& \times \epsilon_{X_{j_{1}}}\left(\omega_{X_{j_{1}}}\right) \ldots \epsilon_{X_{j_{n}}}\left(\omega_{X_{j_{n}}}\right)
\end{aligned}
$$

and

$\mathcal{L}^{X_{j_{1}} \ldots X_{j n}}\left(\omega_{X_{j_{1}}}, \ldots, \omega_{X_{j_{n}}}\right)=\left(\frac{\mathrm{d}^{n} \mathcal{L}}{\mathrm{d} \epsilon_{X_{j_{1}}}\left(\omega_{X_{j_{1}}}\right) \ldots \mathrm{d} \epsilon_{X_{j_{n}}}\left(\omega_{X_{j_{n}}}\right)}\right)_{0}$.

The complex quasi-energy Lagrangian, ${ }^{c} \mathcal{L}$, and its complex conjugate ${ }^{c} \mathcal{L}^{*}$, may be expanded in the same way,

$$
\begin{aligned}
{ }^{c} \mathcal{L} & =\sum_{n}{ }^{c} \mathcal{L}^{(n)}, \\
{ }^{c} \mathcal{L}^{*} & =\sum_{n}^{n}{ }^{c} \mathcal{L}^{(n)^{*}},
\end{aligned}
$$

where

$$
\begin{aligned}
{ }^{c} \mathcal{L}^{(n)}= & \frac{1}{n !} \sum_{\substack{j_{1} \leq \cdots \leq j_{n}\\
}} \mathcal{N}_{\omega_{X_{j_{1}}} \ldots \omega_{X_{j_{n}}}}^{X_{j_{1} \ldots X_{j_{n}}}}{ }^{c} \mathcal{L}^{X_{j_{1}} \ldots X_{j_{n}}}\left(\omega_{X_{j_{1}}}, \ldots, \omega_{X_{j_{n}}}\right) \\
& \times \epsilon_{X_{j_{1}}}\left(\omega_{X_{j_{1}}}\right) \ldots \epsilon_{X_{j_{n}}}\left(\omega_{X_{j_{n}}}\right)
\end{aligned}
$$

$$
\begin{aligned}
{ }^{c} \mathcal{L}^{(n)^{*}}= & \frac{1}{n !} \sum_{j_{1} \leq \cdots \leq j_{n}} \\
& \times \mathcal{N}_{\omega_{X_{j_{1}}} \ldots \omega_{X_{j_{n}}}}^{X_{j_{1}} \ldots X_{j_{n}}}{ }^{c} \mathcal{L}^{X_{j_{1}} \ldots X_{j_{n}} *}\left(-\omega_{X_{j_{1}}}, \ldots,-\omega_{X_{j_{n}}}\right) \\
& \times \epsilon_{X_{j_{1}}}\left(\omega_{X_{j_{1}}}\right) \ldots \epsilon_{X_{j_{n}}}\left(\omega_{X_{j_{n}}}\right),
\end{aligned}
$$

and

$$
\begin{aligned}
& { }^{c} \mathcal{L}^{X_{j_{1}} \ldots X_{j_{n}}}\left(\omega_{X_{j_{1}}}, \ldots, \omega_{X_{j_{n}}}\right) \\
& =\left(\frac{\mathrm{d}^{n}{ }^{c} \mathcal{L}}{\mathrm{d} \epsilon_{X_{j_{1}}}\left(\omega_{X_{j_{1}}}\right) \ldots \mathrm{d} \epsilon_{X_{j_{n}}}\left(\omega_{X_{j_{n}}}\right)}\right),
\end{aligned}
$$

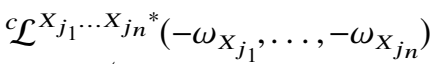

$$
\begin{aligned}
& =\left(\frac{\mathrm{d}^{n} \mathcal{L}^{*}}{\mathrm{~d} \epsilon_{X_{j_{1}}}\left(\omega_{X_{j_{1}}}\right) \ldots \mathrm{d} \epsilon_{X_{j_{n}}}\left(\omega_{X_{j_{n}}}\right)}\right)_{0} .
\end{aligned}
$$

Note that

$$
{ }^{c} \mathcal{L}^{(0)}=L_{0}
$$

is given in Eq. (194). To obtain Eq. (233), we take the complex conjugate of Eq. (232) with no restriction on the summation indices,

$$
\begin{aligned}
& { }^{c} \mathcal{L}^{(n)^{*}}=\frac{1}{n !} \sum_{j_{1} \cdots j_{n}}\left[{ }^{c} \mathcal{L}^{X_{j_{1}} \ldots X_{j_{n}}}\left(\omega_{X_{j_{1}}}, \ldots, \omega_{X_{j_{n}}}\right)\right]^{*} \epsilon_{X_{j_{1}}}^{*}\left(\omega_{X_{j_{1}}}\right) \ldots \epsilon_{X_{j_{n}}}^{*}\left(\omega_{X_{j_{n}}}\right)=\frac{1}{n !} \sum_{j_{1} \cdots j_{n}}{ }^{c} \mathcal{L}^{X_{j_{1}} \ldots X_{j_{n}}{ }^{*}}\left(\omega_{X_{j_{1}}}, \ldots, \omega_{X_{j_{n}}}\right)
\end{aligned}
$$

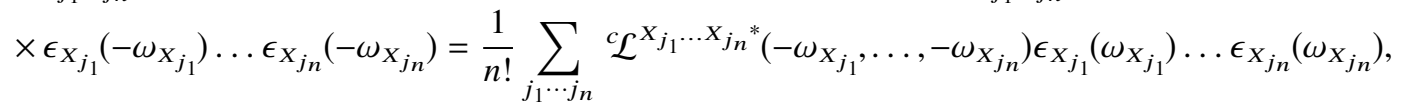

where to obtain the second equality we have used Eq. (4) and to obtain the last equality we have used that for each summation index $j_{k}$ both $\epsilon_{X_{j_{k}}}\left(\omega_{X_{j_{k}}}\right)$ and $\epsilon_{X_{j_{k}}}\left(-\omega_{X_{j_{k}}}\right)$ enter the expansion. From Eq. (237), we straightforwardly obtain Eq. (233).

We will now express response functions in terms of ${ }^{c} \mathcal{L}^{X_{j_{1}} \ldots X_{j_{n}}}\left(\omega_{X_{j_{1}}}, \ldots, \omega_{X_{j_{n}}}\right)$. We initially insert Eq. (215) in Eq. (214) giving

$\frac{\mathrm{d} \mathcal{E}}{\mathrm{d} \epsilon_{X_{j_{1}}}\left(\omega_{X_{j_{1}}}\right)}=\frac{1}{2}\left(\frac{\mathrm{d}^{c} \mathcal{L}}{\mathrm{d} \epsilon_{X_{j_{1}}}\left(\omega_{X_{j_{1}}}\right)}+\frac{\mathrm{d}^{c} \mathcal{L}^{*}}{\mathrm{~d} \epsilon_{X_{j_{1}}}\left(\omega_{X_{j_{1}}}\right)}\right)$.

Differentiating both sides of Eq. (238) with respect to the perturbation strengths $\epsilon_{X_{j_{2}}}\left(\omega_{X_{j_{2}}}\right), \ldots, \epsilon_{X_{j_{n}}}\left(\omega_{X_{j_{n}}}\right)$ and setting all perturbation strengths to zero gives

$$
\begin{aligned}
& \left(\frac{\mathrm{d}^{n} \mathcal{E}}{\mathrm{d} \epsilon_{X_{j_{1}}}\left(\omega_{X_{j_{1}}}\right) \ldots \mathrm{d} \epsilon_{X_{j_{n}}}\left(\omega_{X_{j n}}\right)}\right)_{0} \\
& =\frac{1}{2}\left[\left(\frac{\mathrm{d}^{n} \mathcal{L}}{\mathrm{d} \epsilon_{X_{j_{1}}}\left(\omega_{X_{j_{1}}}\right) \ldots \mathrm{d} \epsilon_{X_{j n}}\left(\omega_{X_{j n}}\right)}\right)_{0}\right. \\
& \left.+\left(\frac{\mathrm{d}^{n} \mathcal{L}^{*}}{\mathrm{~d} \epsilon_{X_{j_{1}}}\left(\omega_{X_{j_{1}}}\right) \ldots \mathrm{d} \epsilon_{X_{j n}}\left(\omega_{X_{j n}}\right)}\right)_{0}\right] .
\end{aligned}
$$

Using Eqs. (65) and (170), we obtain

$$
\left\langle\left\langle X_{j_{1}} ; X_{j_{2}}, \ldots, X_{j_{n}}\right\rangle\right\rangle_{\omega_{X_{j_{2}}}, \ldots, \omega_{X_{j n}}}
$$

$$
\begin{aligned}
= & \frac{1}{2}\left[\left(\frac{\mathrm{d}^{n} \mathcal{L}}{\mathrm{d} \epsilon_{X_{j_{1}}}\left(\omega_{X_{j_{1}}}\right) \ldots \mathrm{d} \epsilon_{X_{j n}}\left(\omega_{X_{j n}}\right)}\right)_{0}\right. \\
& \left.+\left(\frac{\mathrm{d}^{n} \mathcal{L}^{*}}{\mathrm{~d} \epsilon_{X_{j_{1}}}\left(\omega_{X_{j_{1}}}\right) \ldots \mathrm{d} \epsilon_{X_{j_{n}}}\left(\omega_{X_{j n}}\right)}\right)_{0}\right], \\
& \sum_{m=1}^{n} \omega_{X_{j m}}=0 .
\end{aligned}
$$

Inserting Eqs. (234) and (235) in Eq. (240) gives

$$
\begin{aligned}
& \left\langle\left\langle X_{j_{1}} ; X_{j_{2}}, \ldots, X_{j_{n}}\right\rangle\right\rangle_{\omega_{X_{j_{2}}}, \ldots, \omega_{X_{j_{n}}}}
\end{aligned}
$$

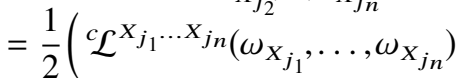

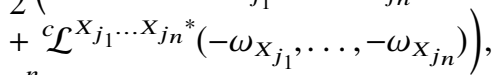

$$
\begin{aligned}
& \sum_{m=1}^{n} \omega_{X_{j m}}=0 \text {. }
\end{aligned}
$$

Introducing the complex conjugation and frequency sign inversion operator $C^{ \pm \omega}$ defined as

$$
\begin{aligned}
C^{ \pm \omega} h^{X_{j_{1}} \cdots X_{j_{n}}}\left(\omega_{X_{j_{1}}}, \ldots, \omega_{X_{j_{n}}}\right) \\
=h^{X_{j_{1}} \cdots X_{j_{n}}}\left(\omega_{X_{j_{1}}}, \ldots, \omega_{X_{j_{n}}}\right) \\
\quad+h^{X_{j_{1}} \cdots X_{j_{n}}{ }^{*}}\left(-\omega_{X_{j_{1}}}, \ldots,-\omega_{X_{j_{n}}}\right),
\end{aligned}
$$

where $h^{X_{j_{1}} \cdots X_{j_{n}}}\left(\omega_{X_{j_{1}}}, \ldots, \omega_{X_{j_{n}}}\right)$ is an arbitrary complex function of the set of operator-frequency pairs, we may write 
Eq. (241) in a compact manner as

$$
\begin{aligned}
\left\langle\left\langle X_{j_{1}} ; X_{j_{2}}, \ldots, X_{j_{n}}\right\rangle\right\rangle_{\omega_{X_{j_{2}}}, \ldots, \omega_{X_{j n}}} & \\
\quad= & \frac{1}{2} C^{ \pm \omega} \mathcal{L}^{X_{j_{1}} \ldots X_{j_{n}}}\left(\omega_{X_{j_{1}}}, \ldots, \omega_{X_{j_{n}}}\right), \quad \sum_{m=1}^{n} \omega_{X_{j_{m}}}=0 .
\end{aligned}
$$

Following the same route as for deriving $\mathcal{E}^{X_{j_{1}} \ldots X_{j_{n}}}$ $\left(\omega_{X_{j_{1}}}, \ldots, \omega_{X_{j n}}\right)$ of Eq. (146), we may identify ${ }^{c} \mathcal{L}^{X_{j_{1}} \ldots X_{j_{n}}}$ $\left(\omega_{X_{j_{1}}}, \ldots, \omega_{X_{j_{n}}}\right)$ from Eq. (219) in terms of the symmetry distinct contributions, ${ }^{c} \widetilde{f}_{\omega_{X_{j_{1}}} \ldots \omega_{X_{j n}} \ldots X_{j_{n}}}$,

$$
\begin{aligned}
& { }^{c} \mathcal{L}^{X_{j_{1}} \ldots X_{j n}}\left(\omega_{X_{j_{1}}}, \ldots, \omega_{X_{j_{n}}}\right)=P_{\omega_{X_{j_{1}}} \ldots \omega_{X_{j_{n}}}}^{X_{j_{1}} \ldots X_{j_{n}}} c \widetilde{f}_{\omega_{X_{j_{1}}} \ldots \omega_{X_{j_{n}}} \ldots X_{j_{n}}}, \\
& \sum_{m=1}^{n} \omega_{X_{j m}}=0 .
\end{aligned}
$$

When the Hermitian eigenvalue equation is solved via projection, molecular response functions are thus determined from Eq. (243), where the complex quasi-energy Lagrangian is obtained from Eq. (244).

\section{Stationary conditions for the perturbation components of the quasi-energy Lagrangian}

The individual components of the complex quasi-energy Lagrangian, ${ }^{c} \mathcal{L}^{X_{j_{1}} \cdots X_{j_{n}}}\left(\omega_{X_{j_{1}}}, \ldots, \omega_{X_{j n}}\right)$, are stationary with respect to the components of the wave-function parameters, $b_{i}^{X_{j_{1}} \cdots X_{j_{m}}}\left(\omega_{X_{j_{1}}}, \ldots, \omega_{X_{j_{m}}}\right)$, and multipliers, $\bar{b}_{i}^{X_{j_{1}} \cdots X_{j_{m}}}$ $\left(\omega_{X_{j_{1}}}, \ldots, \omega_{X_{j_{m}}}\right)$, when the condition $\sum_{m=1}^{n} \omega_{X_{j_{m}}}=0$ is satisfied,

$$
\begin{aligned}
& \frac{\partial \mathcal{L}^{X_{j_{1}} \ldots X_{j n}}\left(\omega_{X_{j_{1}}}, \ldots, \omega_{X_{j_{n}}}\right)}{\partial b_{i}^{X_{j_{1}} \cdots X_{j_{m}}}\left(\omega_{X_{j_{1}}}, \ldots, \omega_{X_{j m}}\right)}=0, \quad \sum_{k=1}^{n} \omega_{X_{j_{k}}}=0, \\
& \frac{\partial{ }^{c} \mathcal{L}^{X_{j_{1}} \ldots X_{j n}}\left(\omega_{X_{j_{1}}}, \ldots, \omega_{X_{j n}}\right)}{\partial \bar{b}_{i}^{X_{j_{1}} \cdots X_{j m}}\left(\omega_{X_{j_{1}}}, \ldots, \omega_{X_{j m}}\right)}=0, \quad \sum_{k=1}^{n} \omega_{X_{j_{k}}}=0 .
\end{aligned}
$$

To show this, we consider the derivative of ${ }^{c} \mathcal{L}^{X_{j_{1}} \cdots X_{j_{n}}}\left(\omega_{X_{j_{1}}}, \ldots, \omega_{X_{j_{n}}}\right)$ in Eq. (234),

$$
\begin{aligned}
\frac{\partial^{c} \mathcal{L}^{X_{j_{1}} \ldots X_{j n}}\left(\omega_{X_{j_{1}}}, \ldots, \omega_{X_{j n}}\right)}{\partial b_{i}^{X_{j_{1}} \cdots X_{j m}}\left(\omega_{X_{j_{1}}}, \ldots, \omega_{X_{j m}}\right)} & =\frac{\partial}{\partial b_{i}^{X_{j_{1}} \cdots X_{j m}}\left(\omega_{X_{j_{1}}}, \ldots, \omega_{X_{j m}}\right)}\left(\frac{\mathrm{d}^{n} \mathcal{L}}{\mathrm{d} \epsilon_{X_{j_{1}}}\left(\omega_{X_{j_{1}}}\right) \ldots \epsilon_{X_{j n}}\left(\omega_{X_{j_{n}}}\right)}\right)_{0} \\
& =\left(\frac{\mathrm{d}^{n}}{\mathrm{~d} \epsilon_{X_{j_{1}}}\left(\omega_{X_{j_{1}}}\right) \ldots \epsilon_{X_{j n}}\left(\omega_{X_{j n}}\right)} \frac{\partial^{c} \mathcal{L}}{\partial b_{i}^{X_{j_{1}} \cdots X_{j m}}\left(\omega_{X_{j_{1}}}, \ldots, \omega_{X_{j_{m}}}\right)}\right)_{0} \\
& =\left(\frac{\mathrm{d}^{n}}{\mathrm{~d} \epsilon_{X_{j_{1}}}\left(\omega_{X_{j_{1}}}\right) \ldots \epsilon_{X_{j_{n}}}\left(\omega_{X_{j_{n}}}\right)} \sum_{k} \sum_{L} \frac{\partial^{c} \mathcal{L}}{\partial b_{k}\left(\omega_{L}\right)} \frac{\partial b_{k}\left(\omega_{L}\right)}{\partial b_{i}^{X_{j_{1}} \cdots X_{j_{m}}}\left(\omega_{X_{j_{1}}}, \ldots, \omega_{X_{j_{m}}}\right)}\right)_{0},
\end{aligned}
$$

where in the last equality we have used the chain rule. Applying the stationary condition of Eq. (225) in Eq. (247), we conclude that the individual components of the quasi-energy Lagrangian, $\mathcal{L}^{X_{j_{1}} \cdots X_{j_{n}}}\left(\omega_{X_{j_{1}}}, \ldots, \omega_{X_{j_{n}}}\right)$, are stationary with respect to variations in the individual components of the coefficients, $b_{i}^{X_{j_{1}} \cdots X_{j m}}\left(\omega_{X_{j_{1}}}, \ldots, \omega_{X_{j m}}\right)$, as expressed by Eq. (245). A similar derivation shows that the stationary condition in Eq. (246) is fulfilled for multipliers. Equations (245) and (246) may be used to obtain response equations that determine the Lagrangian multipliers, $\bar{b}_{i}^{X_{j_{1}} \cdots X_{j m}}$, and the wave-function coefficients, $b_{i}^{X_{j_{1} \cdots X_{j m}}}$, respectively.

\section{QUASI-ENERGY LAGRANGIANS FOR THE CI, CC-CI AND CC WAVE FUNCTION-MODELS}

In this section, we introduce the $\mathrm{CI}, \mathrm{CC}-\mathrm{CI}$, and $\mathrm{CC}$ wave-function models and determine the response functions for these models using projection. For the CI model, we use a linear parametrization for both the unperturbed and the perturbed system. For the CC model, we use an exponential parametrization for both the unperturbed and the perturbed system, whereas for the CC-CI model, an exponential parametrization is used for the unperturbed system and a linear parametrization for the perturbed system. In Sec. $\mathrm{V}$ A, the CI, CC-CI, and CC models are introduced, and in
Sec. V B, we show how simplifications may be introduced in ${ }^{c} \mathcal{L}_{H_{0}}$ [Eq. (220)], ${ }^{c} \mathcal{L}_{F}$ [Eq. (221)], and ${ }^{c} \mathcal{L}_{X_{j_{1}}}$ [Eq. (222)] for these wave function models. In particular, we use that the zero-order amplitude and multiplier equations [Eqs. (195) and (196)] are satisfied to simplify the expression for ${ }^{c} \mathcal{L}_{H_{0}}$ and identify a generic expression for the quasi-energy Lagrangian for the CI, CC-CI, and CC models. Response functions for the CI, CC-CI, and CC models are given in Secs. VI-VIII.

\section{A. Wave function models in terms of different parametrizations of the unperturbed state and the time evolution}

We start out by introducing the notation we will use for $B_{0}$ [Eq. (179)] and $B(t)$ [Eq. (201)] when these operators refer to either a linear or an exponential parametrization. For the linear parametrization [Eq. (180)], we use the operator label $S$ and the amplitudes $b_{k}$ and the multipliers $\bar{b}_{k}$ will be denoted $s_{k}$ and $\bar{s}_{k}$, respectively,

$$
B=S=\sum_{k} s_{k}|k\rangle\langle\mathscr{R}| .
$$

Equation (248) implies that

$$
S^{2}(t)=0
$$


For the exponential parametrization [Eq. (183)], we assume that $|\mathscr{R}\rangle=|\mathrm{HF}\rangle$ is the Hartree-Fock state and relabel the manybody orbital excitation operators of Eq. (183) as $\tau_{\mu_{k}}$, where $k$ denotes an excitation level and $\mu_{k}$ is an excitation at this excitation level. The orthogonal complement set of states then becomes (cf. Eq. (185))

$$
\left|\mu_{k}\right\rangle=\tau_{\mu_{k}}|\mathrm{HF}\rangle .
$$

We use the $T, t_{\mu_{k}}$, and $\bar{t}_{\mu_{k}}$ symbols for the exponential parametrization,

$$
B=T=\sum_{\mu_{k}} t_{\mu_{k}} \tau_{\mu_{k}} .
$$

Four wave function models may be obtained from Eq. (198),

$$
\begin{gathered}
\left|0_{I}^{\mathrm{CI}}\right\rangle=\exp \left(S_{0}\right) \exp (S(t))|\mathscr{R}\rangle, \\
\left|0_{I}^{\mathrm{CC}}\right\rangle=\exp \left(T_{0}\right) \exp (T(t))|\mathscr{R}\rangle, \\
\left|0_{I}^{\mathrm{CC}-\mathrm{CI}}\right\rangle=\exp \left(T_{0}\right) \exp (S(t))|\mathscr{R}\rangle, \\
\left|0_{I}^{\mathrm{CI}-\mathrm{CC}}\right\rangle=\exp \left(S_{0}\right) \exp (T(t))|\mathscr{R}\rangle .
\end{gathered}
$$

When the excitation manifolds, $|k\rangle\langle\mathscr{R}|$ and $\tau_{\mu_{k}}$, are complete, the four wave function models [Eqs. (252)-(255)] all describe an exact time evolution and give the same exact result. When the excitation manifolds are truncated, the four models describe different but well-defined time evolutions. In the CC model [Eq. (253)], both the unperturbed state and the time evolution are described using an exponential expansion leading to the well-known advantages of the rapid convergence with the excitation rank and a size-extensive energy for the unperturbed systems. By contrast, the CI model [Eq. (252)], where both the unperturbed state and the time evolution are linearly parametrized, exhibits a slow convergence with the excitation rank and a nonsize-extensive energy for the unperturbed systems. In the CC-CI model [Eq. (254)], the unperturbed state is described with a coupled cluster state and the time evolution of this state by a simple linear CI expansion.
As the time-dependent perturbation is small, it may suffice to describe it using a linear parametrization. In the fourth model [Eq. (255)], the unperturbed state is described using the CI model. The exponential parametrization of the time evolution cannot remedy the deficiencies of the CI model for describing the unperturbed system and this model will not be considered further.

\section{B. Simplifications in the complex quasi-energy Lagrangian in the frequency domain}

In this subsection, we simplify the complex quasi-energy Lagrangian of Eq. (219) to obtain a generic expression that can be applied for the three wave function models CI, CC-CI, and CC. We first transform ${ }^{c} \mathcal{L}_{H_{0}}$ [Eq. (220)], ${ }^{c} \mathcal{L}_{F}$ [Eq. (221)], and ${ }^{c} \mathcal{L}_{X_{j_{1}}}$ [Eq. (222)] to the frequency domain and then simplify the resulting expressions.

Consider first ${ }^{c} \mathcal{L}_{H_{0}}$. Applying the Baker-CampbellHausdorff (BCH) expansion to ${ }^{c} \mathcal{L}_{H_{0}}$ [Eq. (220)] gives

$$
{ }^{c} \mathcal{L}_{H_{0}}={ }^{c} \mathcal{L}_{H_{0}}^{L}+{ }^{c} \mathcal{L}_{H_{0}}^{N L},
$$

where ${ }^{c} \mathcal{L}_{H_{0}}^{L}$ and ${ }^{c} \mathcal{L}_{H_{0}}^{N L}$ denote terms that are linear and nonlinear in the $b_{m}(t)$ coefficients, respectively. ${ }^{c} \mathcal{L}_{H_{0}}^{L}$ reads

$$
\begin{aligned}
{ }^{c} \mathcal{L}_{H_{0}}^{L}= & \left\langle\mathscr{R}\left|H_{0}^{B_{0}}\right| \mathscr{R}\right\rangle+\left\{\left\langle\mathscr{R}\left|\left[H_{0}^{B_{0}}, B(t)\right]\right| \mathscr{R}\right\rangle\right\}_{T} \\
& +\sum_{k K} \bar{b}_{k}\left(\omega_{K}\right)\left\{\left\langle k\left|H_{0}^{B_{0}}\right| \mathscr{R}\right\rangle \exp \left(-i \omega_{K} t\right)\right\}_{T} \\
& +\sum_{k K} \bar{b}_{k}\left(\omega_{K}\right)\left\{\left\langle k\left|\left[H_{0}^{B_{0}}, B(t)\right]\right| \mathscr{R}\right\rangle \exp \left(-i \omega_{K} t\right)\right\}_{T},
\end{aligned}
$$

whereas the explicit form of ${ }^{c} \mathcal{L}_{H_{0}}^{N L}$ depends on whether the time evolution is linearly $(B(t)=S(t))$ or exponentially $(B(t)=T(t))$ parametrized,

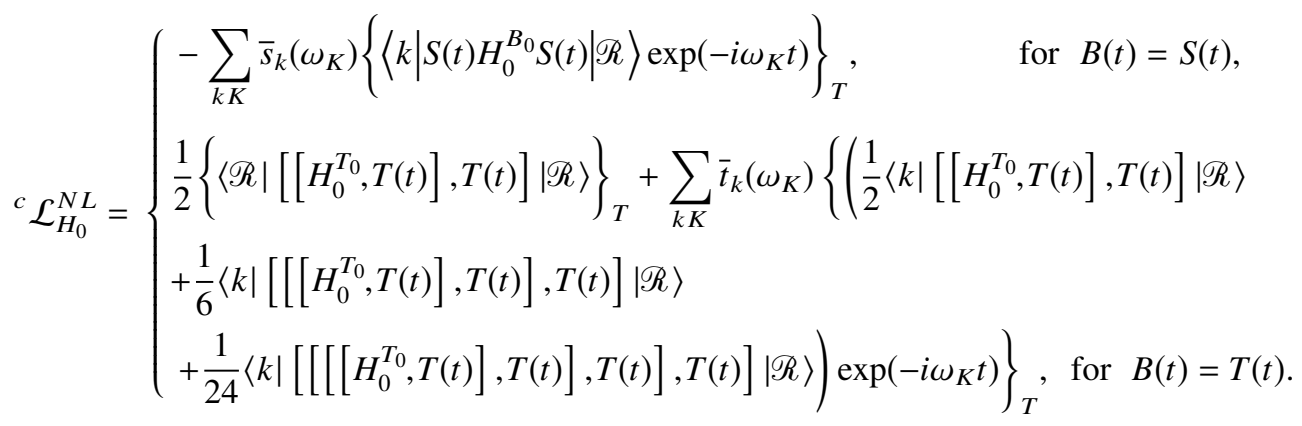

To obtain Eq. (258a), we have used Eq. (249) to truncate the $\mathrm{BCH}$ expansion. In Eq. (258b), $B_{0}$ has been replaced by $T_{0}$ because for an exponential parametrization of the time evolution, the only unperturbed state we consider is exponentially parametrized, as discussed in Subsection V A To obtain Eq. (258b), we have used that $H_{0}$ and $H_{0}^{T_{0}}$ are rank- two operators, and therefore, the $\mathrm{BCH}$ expansion truncates after the quadratic term for $\left\langle\mathscr{R}\left|\exp (-T(t)) H_{0}^{T_{0}} \exp (T(t))\right| \mathscr{R}\right\rangle$ and after the quartic term for $\left\langle k\left|\exp (-T(t)) H_{0}^{T_{0}} \exp (T(t))\right| \mathscr{R}\right\rangle$.

We now transform ${ }^{c} \mathcal{L}_{H_{0}}^{L}$ [Eq. (257)] to the frequency domain. To do that we substitute Eq. (201) in Eq. (257) and then insert Eq. (114) for $b_{m}(t)$, giving 


$$
\begin{aligned}
{ }^{c} \mathcal{L}_{H_{0}}^{L}= & \left\langle\mathscr{R}\left|H_{0}^{B_{0}}\right| \mathscr{R}\right\rangle+\sum_{m M} b_{m}\left(\omega_{M}\right)\left\langle\mathscr{R}\left|\left[H_{0}^{B_{0}}, \beta_{m}\right]\right| \mathscr{R}\right\rangle \\
& \times\left\{\exp \left(-i \omega_{M} t\right)\right\}_{T} \\
& +\sum_{k K} \bar{b}_{k}\left(\omega_{K}\right)\left\langle k\left|H_{0}^{B_{0}}\right| \mathscr{R}\right\rangle\left\{\exp \left(-i \omega_{K} t\right)\right\}_{T} \\
& +\sum_{k m K M} \bar{b}_{k}\left(\omega_{K}\right) b_{m}\left(\omega_{M}\right)\left\langle k\left|\left[H_{0}^{B_{0}}, \beta_{m}\right]\right| \mathscr{R}\right\rangle \\
& \times\left\{\exp \left(-i\left(\omega_{K}+\omega_{M}\right) t\right)\right\}_{T} .
\end{aligned}
$$

Using Eq. (120), the explicit time dependence may be removed from Eq. (259),

$$
\begin{aligned}
{ }^{c} \mathcal{L}_{H_{0}}^{L}= & E_{0}+\sum_{m M} b_{m}\left(\omega_{M}\right)\left\langle\mathscr{R}\left|\left[H_{0}^{B_{0}}, \beta_{m}\right]\right| \mathscr{R}\right\rangle \Delta\left(\omega_{M}\right) \\
& +\sum_{k K} \bar{b}_{k}\left(\omega_{K}\right)\left\langle k\left|H_{0}^{B_{0}}\right| \mathscr{R}\right\rangle \Delta\left(\omega_{K}\right) \\
& +\sum_{k m K M} \bar{b}_{k}\left(\omega_{K}\right) b_{m}\left(\omega_{M}\right)\left\langle k\left|\left[H_{0}^{B_{0}}, \beta_{m}\right]\right| \mathscr{R}\right\rangle \\
& \times \Delta\left(\omega_{K}+\omega_{M}\right),
\end{aligned}
$$

where we have also used Eq. (193a).

We now introduce the Jacobian in Eq. (260). ${ }^{c} \mathcal{L}_{H_{0}}$ contains $b_{m}\left(\omega_{M}\right)$ and $\bar{b}_{k}\left(\omega_{K}\right)$ as independent parameters. The Jacobian therefore becomes [cf. Eq. (126)]

$$
\begin{aligned}
{ }^{P} J_{k m} & =\left(\frac{\partial^{2 c} \mathcal{L}_{H_{0}}}{\partial \bar{b}_{k}\left(\omega_{K}\right) \partial b_{m}\left(\omega_{M}\right)}\right)_{0}=\left(\frac{\partial^{2 c} \mathcal{L}_{H_{0}}^{L}}{\partial \bar{b}_{k}\left(\omega_{K}\right) \partial b_{m}\left(\omega_{M}\right)}\right)_{0} \\
& =\left\langle k\left|\left[H_{0}^{B_{0}}, \beta_{m}\right]\right| \mathscr{R}\right\rangle,
\end{aligned}
$$

where we have used that at zero perturbation strength the $b_{m}\left(\omega_{M}\right)$ coefficients vanish and the frequencies are equal to zero. Inserting Eq. (261) in Eq. (260) gives

$$
\begin{aligned}
{ }^{c} \mathcal{L}_{H_{0}}^{L}= & E_{0}+\sum_{m M} b_{m}\left(\omega_{M}\right)\left\langle\mathscr{R}\left|\left[H_{0}^{B_{0}}, \beta_{m}\right]\right| \mathscr{R}\right\rangle \Delta\left(\omega_{M}\right) \\
& +\sum_{k K} \bar{b}_{k}\left(\omega_{K}\right)\left\langle k\left|H_{0}^{B_{0}}\right| \mathscr{R}\right\rangle \Delta\left(\omega_{K}\right) \\
& +\sum_{k m K M} \bar{b}_{k}\left(\omega_{K}\right) b_{m}\left(\omega_{M}\right)^{P} J_{k m} \Delta\left(\omega_{K}+\omega_{M}\right) .
\end{aligned}
$$

We now separate out the zero-order contribution in the Lagrangian multipliers $\bar{b}_{k}\left(\omega_{K}\right)$,

$$
\bar{b}_{k}\left(\omega_{K}\right)=\bar{b}_{k}^{(0)}+\bar{b}_{k}^{(\backslash 0)}\left(\omega_{K}\right),
$$

where $\bar{b}_{k}^{(0)}$ denotes the zero-order multipliers in Eq. (194) and $\bar{b}_{k}^{(\backslash 0)}\left(\omega_{K}\right)$ denotes multipliers that do not contain the zero-order contribution. Inserting Eq. (263) in the last term of Eq. (262) gives

$$
\begin{aligned}
{ }^{c} \mathcal{L}_{H_{0}}^{L}= & E_{0}+\sum_{m M} b_{m}\left(\omega_{M}\right)\left(\left\langle\mathscr{R}\left|\left[H_{0}^{B_{0}}, \beta_{m}\right]\right| \mathscr{R}\right\rangle\right. \\
& \left.+\sum_{k} \bar{b}_{k}^{(0)} P^{P} J_{k m}\right) \Delta\left(\omega_{M}\right)
\end{aligned}
$$

$$
\begin{aligned}
& +\sum_{k K} \bar{b}_{k}\left(\omega_{K}\right)\left\langle k\left|H_{0}^{B_{0}}\right| \Re\right\rangle \Delta\left(\omega_{K}\right) \\
& +\sum_{k m K M} \bar{b}_{k}^{(\backslash 0)}\left(\omega_{K}\right) b_{m}\left(\omega_{M}\right)^{P} J_{k m} \Delta\left(\omega_{K}+\omega_{M}\right) .
\end{aligned}
$$

We now show that ${ }^{c} \mathcal{L}_{H_{0}}^{L}$ may be simplified to

$$
\begin{aligned}
{ }^{c} \mathcal{L}_{H_{0}}^{L}= & E_{0}+\sum_{k m K M} \bar{b}_{k}^{(\backslash 0)}\left(\omega_{K}\right) b_{m}\left(\omega_{M}\right){ }^{P} J_{k m} \\
& \times \Delta\left(\omega_{K}+\omega_{M}\right)
\end{aligned}
$$

using the amplitudes equation [Eq. (195)] and the Lagrangian multipliers equation [Eq. (196)] for the unperturbed system.

To obtain Eq. (265), we initially note that the third term in Eq. (264) contains the zero-order amplitude equation [Eq. (195)] and vanishes for all the three models: CI, CC, and CC-CI. The second term of Eq. (264) contains the Jacobian of Eq. (261). For the CI and CC models, the operators $\beta_{n}$ describing the unperturbed system are identical to the operators describing the time evolution. The zero-order Lagrangian multipliers equation [Eq. (196)] may therefore be invoked to show that the second term in Eq. (264) vanishes for the $\mathrm{CI}$ and CC models.

To see that the second term vanishes also for the CCCI model, we may write for this model the terms in the parentheses as

$$
\begin{array}{r}
\left\langle\mathrm{HF}\left|\left[H_{0}^{T_{0}},\left|v_{m}\right\rangle\langle\mathrm{HF}|\right]\right| \mathrm{HF}\right\rangle+\sum_{\mu_{k}} \bar{t}_{\mu_{k}}^{(0)} \mathrm{CC}-\mathrm{CI} J_{\mu_{k} \nu_{m}} \\
=\left\langle\mathrm{HF}\left|H_{0}^{T_{0}}\right| v_{m}\right\rangle+\sum_{\mu_{k}} \bar{t}_{\mu_{k}}^{(0)} \mathrm{CC}-\mathrm{CI} J_{\mu_{k} v_{m}},
\end{array}
$$

where we have used that in the CC-CI model $B_{0}=T_{0}$ and the $|\mathcal{B}\rangle$ basis [Eq. (173)] reads $\left\{|\mathrm{HF}\rangle,\left|\mu_{k}\right\rangle\right\}$, and where [cf. Eq. (261)]

$$
\begin{aligned}
{ }^{\mathrm{CC}-\mathrm{CI}} J_{\mu_{k} v_{m}} & =\left\langle\mu_{k}\left|\left[H_{0}^{T_{0}},\left|v_{m}\right\rangle\langle\mathrm{HF}|\right]\right| \mathrm{HF}\right\rangle \\
& =\left\langle\mu_{k}\left|H_{0}^{T_{0}}\right| v_{m}\right\rangle-\delta_{\mu_{k} v_{m}}\left\langle\mathrm{HF}\left|H_{0}^{T_{0}}\right| \mathrm{HF}\right\rangle .
\end{aligned}
$$

In the CC-CI model, we use the CC state as the unperturbed state and the cluster amplitudes therefore satisfy the zero-order multiplier equation [see Eq. (196)]

$$
\begin{aligned}
0 & =\left\langle\mathrm{HF}\left|\left[H_{0}^{T_{0}}, \tau_{v_{m}}\right]\right| \mathrm{HF}\right\rangle+\sum_{\mu_{k}} \bar{t}_{\mu_{k}}^{(0)}\left\langle\mu_{k}\left|\left[H_{0}^{T_{0}}, \tau_{v_{m}}\right]\right| \mathrm{HF}\right\rangle \\
& =\left\langle\mathrm{HF}\left|H_{0}^{T_{0}}\right| v_{m}\right\rangle+\sum_{\mu_{k}} \bar{t}_{\mu_{k}}^{(0)}{ }^{\mathrm{CC}-\mathrm{CI}} J_{\mu_{k} v_{m}},
\end{aligned}
$$

where we have used

$$
\begin{aligned}
\left\langle\mu_{k}\left|\left[H_{0}^{T_{0}}, \tau_{v_{m}}\right]\right| \mathrm{HF}\right\rangle & =\left\langle\mu_{k}\left|H_{0}^{T_{0}}\right| v_{m}\right\rangle-\delta_{\mu_{k} v_{m}}\left\langle\mathrm{HF}\left|H_{0}^{T_{0}}\right| \mathrm{HF}\right\rangle \\
& ={ }^{\mathrm{CC}-\mathrm{CI}} J_{\mu_{k} v_{m}} .
\end{aligned}
$$

To obtain the second equality in Eq. (269), we have used that $\left\langle\mu_{k}\left|\tau_{v_{m}} H_{0}^{T_{0}}\right| \mathrm{HF}\right\rangle$ vanishes for $k<m$ due to Eq. (187) and for $k>m$ it becomes $\left\langle\mu_{n}\left|H_{0}^{T_{0}}\right| \mathrm{HF}\right\rangle$, where $n<k$, and also vanishes since the zero-order stationary condition of Eq. (195) 
is satisfied. The last equality in Eq. (269) is obtained using Eq. (267). It follows from Eqs. (266) and (268) that the second term in Eq. (264) vanishes also for the CC-CI wave function.
Turning our attention to ${ }^{c} \mathcal{L}_{H_{0}}^{N L}$ [Eq. (258)], we may transform it to the frequency domain, using Eqs. (114), (120), and (201), as

$$
{ }^{c} \mathcal{L}_{H_{0}}^{N L}=\left\{\begin{array}{l}
-\sum_{K L M} \sum_{k} \bar{s}_{k}\left(\omega_{K}\right) s_{k}\left(\omega_{L}\right) \sum_{m} s_{m}\left(\omega_{M}\right)\left\langle\mathscr{R}\left|H_{0}^{B}\right| m\right\rangle \Delta\left(\omega_{K}+\omega_{L}+\omega_{M}\right), \text { for } B(t)=S(t), \\
\frac{1}{2} \sum_{\substack{v_{m} \lambda_{n} \\
M N}} t_{v_{m}}\left(\omega_{M}\right) t_{\lambda_{n}}\left(\omega_{N}\right)\left\langle\mathscr{R}\left|\left[\left[H_{0}^{T_{0}}, \tau_{v_{m}}\right], \tau_{\lambda_{n}}\right]\right| \mathscr{R}\right\rangle \Delta\left(\omega_{M}+\omega_{N}\right) \\
+\frac{1}{2} \sum_{\substack{k v_{m} \lambda_{n} \\
K M N}} \bar{t}_{k}\left(\omega_{K}\right) t_{v_{m}}\left(\omega_{M}\right) t_{\lambda_{n}}\left(\omega_{N}\right)\left\langle k\left|\left[\left[H_{0}^{T_{0}}, \tau_{v_{m}}\right], \tau_{\lambda_{n}}\right]\right| R\right\rangle \Delta\left(\omega_{K}+\omega_{M}+\omega_{N}\right) \\
\left.+\frac{1}{6} \sum_{\substack{k v_{m} \lambda_{n} \sigma_{p} \\
K M N P}} \bar{t}_{k}\left(\omega_{K}\right) t_{v_{m}}\left(\omega_{M}\right) t_{\lambda_{n}}\left(\omega_{N}\right) t_{\sigma_{p}}\left(\omega_{P}\right)\langle k|\left[\left[H_{0}^{T_{0}}, \tau_{v_{m}}\right], \tau_{\lambda_{n}}\right], \tau_{\sigma_{p}}\right]|\mathscr{R}\rangle \\
\times \Delta\left(\omega_{K}+\omega_{M}+\omega_{N}+\omega_{P}\right) \\
\left.+\frac{1}{24} \sum_{\substack{k v_{m} \lambda_{n} \sigma_{p} \rho_{q} \\
K M N P Q}} \bar{t}_{k}\left(\omega_{K}\right) t_{v_{m}}\left(\omega_{M}\right) t_{\lambda_{n}}\left(\omega_{N}\right) t_{\sigma_{p}}\left(\omega_{P}\right) t_{\rho_{q}}\left(\omega_{Q}\right)\langle k|\left[\left[\left[H_{0}^{T_{0}}, \tau_{v_{m}}\right], \tau_{\lambda_{n}}\right], \tau_{\sigma_{p}}\right], \tau_{\rho_{q}}\right]|\mathscr{R}\rangle \\
\times \Delta\left(\omega_{K}+\omega_{M}+\omega_{N}+\omega_{P}+\omega_{Q}\right), \text { for } B(t)=T(t) .
\end{array}\right.
$$

${ }^{c} \mathcal{L}_{F}$ may be transformed to the frequency domain substituting Eq. (119) in Eq. (221),

$$
\begin{aligned}
{ }^{c} \mathcal{L}_{F}= & -\sum_{k m K M} \omega_{M} \bar{b}_{k}\left(\omega_{K}\right) b_{m}\left(\omega_{M}\right)\left\langle k\left|\beta_{m}\right| \mathscr{R}\right\rangle \\
& \times\left\{\exp \left(-i\left(\omega_{K}+\omega_{M}\right) t\right)\right\}_{T} \\
= & -\sum_{k m K M} \omega_{M} \bar{b}_{k}\left(\omega_{K}\right) b_{m}\left(\omega_{M}\right)\left\langle k\left|\beta_{m}\right| \mathscr{R}\right\rangle \Delta\left(\omega_{K}+\omega_{M}\right) \\
= & -\sum_{k K M} \omega_{M} \bar{b}_{k}\left(\omega_{K}\right) b_{k}\left(\omega_{M}\right) \Delta\left(\omega_{K}+\omega_{M}\right)
\end{aligned}
$$

where to obtain the last equality we have used Eq. (185) and the orthonormality of the $|\mathcal{B}\rangle$ set [Eq. (173)].

Considering the ${ }^{c} \mathcal{L}_{X_{j_{1}}}$ contribution [Eq. (222)], we note that as long as $X_{j_{k}}$ are assumed to be one-electron operators, the $\mathrm{BCH}$ expansion truncates at the same order for both linear and exponential parametrization of the time evolution, giving

$$
\begin{aligned}
{ }^{c} \mathcal{L}_{X_{j_{1}}}= & \left\langle\mathscr{R}\left|X_{j_{1}}^{B_{0}}\right| \mathscr{R}\right\rangle \Delta\left(\omega_{X_{j_{1}}}\right)+\sum_{\substack{m \\
\text {. }}} b_{m}\left(\omega_{M}\right)\langle\mathscr{R}| \\
& \times\left[X_{j_{1}}^{B_{0}}, \beta_{m}\right]|\mathscr{R}\rangle \Delta\left(\omega_{X_{j_{1}}}+\omega_{M}\right) \\
& +\sum_{k} \bar{b}_{k}\left(\omega_{K}\right)\left\langle k\left|X_{j_{1}}^{B_{0}}\right| \mathscr{R}\right\rangle \Delta\left(\omega_{X_{j_{1}}}+\omega_{K}\right) \\
& +\sum_{k m} \bar{b}_{k}\left(\omega_{K}\right) b_{m}\left(\omega_{M}\right)\left\langle k\left|\left[X_{j_{1}}^{B_{0}}, \beta_{m}\right]\right| \mathscr{R}\right\rangle \\
& \times \Delta\left(\omega_{X_{j_{1}}}+\omega_{K}+\omega_{M}\right)+\frac{1}{2} \sum_{k m n} \bar{b}_{k}\left(\omega_{K}\right) b_{m}\left(\omega_{M}\right) \\
& \times b_{n}\left(\omega_{N}\right)\left\langle k\left|\left[\left[X_{j_{1}}^{B_{0}}, \beta_{m}\right], \beta_{n}\right]\right| \mathscr{R}\right\rangle \\
& \times \Delta\left(\omega_{X_{j_{1}}}+\omega_{K}+\omega_{M}+\omega_{N}\right) .
\end{aligned}
$$

For convenience in derivation of response functions, we combine ${ }^{c} \mathcal{L}_{H_{0}}$ and ${ }^{c} \mathcal{L}_{F}$ in ${ }^{c} \mathcal{L}_{\mathcal{H}}$,

$$
\begin{aligned}
{ }^{c} \mathcal{L}_{\mathcal{H}}= & { }^{c} \mathcal{L}_{H_{0}}+{ }^{c} \mathcal{L}_{F} \\
= & E_{0}+\sum_{k m K M} \bar{b}_{k}^{(\backslash 0)}\left(\omega_{K}\right) b_{m}\left(\omega_{M}\right)\left({ }^{P} J_{k m}-\omega_{M} \delta_{k m}\right) \\
& \times \Delta\left(\omega_{K}+\omega_{M}\right)+{ }^{c} \mathcal{L}_{H_{0}}^{N L},
\end{aligned}
$$

where in the second term ${ }^{c} \mathcal{L}_{F}$ [Eq. (271)] is combined with the second term of ${ }^{c} \mathcal{L}_{H_{0}}^{L}$ in Eq. (265) and where ${ }^{c} \mathcal{L}_{H_{0}}^{N L}$ is given in Eq. (270). The quasi-energy Lagrangian in Eq. (219) may thus be written as

$$
{ }^{c} \mathcal{L}={ }^{c} \mathcal{L}_{\mathcal{H}}+\sum_{j_{1}}{ }^{c} \mathcal{L}_{X_{j_{1}}} \epsilon_{X_{j_{1}}}\left(\omega_{X_{j_{1}}}\right),
$$

where ${ }^{c} \mathcal{L}_{\mathcal{H}}$ and ${ }^{c} \mathcal{L}_{X_{j_{1}}}$ are given in Eqs. (273) and (272), respectively.

\section{MOLECULAR PROPERTIES FOR THE CI MODEL}

We consider in this section how molecular response functions are derived for the CI model where projection is applied to the time-dependent Schrödinger equation to determine the time evolution. Projection is also used to determine the unperturbed ground state.

\section{A. Parametrization of the $\mathrm{Cl}$ model}

To derive molecular response properties for the CI model we use, as in Sec. III (see Eq. (89)), the ground state for the unperturbed system as the expansion point for the perturbation-induced time development. The basis $|\mathcal{B}\rangle$ [Eq. (173)], in which we describe the time development, 
therefore becomes

$$
|\mathcal{B}\rangle=\left\{\left|0_{0}^{\mathrm{CI}}\right\rangle,\left|\mu_{k}^{\mathrm{CI}}\right\rangle\right\},
$$

where $\left|0_{0}^{\mathrm{CI}}\right\rangle$ is the unit-normalized unperturbed ground state satisfying

$$
H_{0}\left|0_{0}^{\mathrm{CI}}\right\rangle=E_{0}^{\mathrm{CI}}\left|0_{0}^{\mathrm{CI}}\right\rangle
$$

and $\left\{\left|\mu_{k}^{\mathrm{CI}}\right\rangle\right\}$ denotes the orthogonal complement set of states to $\left|0_{0}^{\mathrm{CI}}\right\rangle$. The orthogonal complement states $\left|\mu_{k}^{\mathrm{CI}}\right\rangle$ may or may not be the exact solution to the Schrödinger equation for the unperturbed system [Eq. (67)]. Since the unperturbed ground state, $\left|0_{0}^{\mathrm{CI}}\right\rangle$, is used as the reference state, the intermediate normalized state in Eq. (181) may be expressed as

$$
\begin{aligned}
\left|0_{I_{0}}^{\mathrm{CI}}\right\rangle & =\exp \left(\sum_{\mu_{k}} s_{\mu_{k}}^{(0)}\left|\mu_{k}^{\mathrm{CI}}\right\rangle\left\langle 0_{0}^{\mathrm{CI}}\right|\right)\left|0_{0}^{\mathrm{CI}}\right\rangle \\
& =\left|0_{0}^{\mathrm{CI}}\right\rangle+\sum_{\mu_{k}} s_{\mu_{k}}^{(0)}\left|\mu_{k}^{\mathrm{CI}}\right\rangle .
\end{aligned}
$$

As we consider the time evolution with $\left|0_{0}^{\mathrm{CI}}\right\rangle$ as an expansion point, the $s_{\mu_{k}}^{(0)}$ coefficients, and hence $B_{0}$, become zero. We may, therefore, replace the similarity-transformed Hamiltonian, $H_{0}^{B_{0}}$ [Eq. (191)], by the bare Hamiltonian, $H_{0}$, in the complex quasi-energy Lagrangian expression [Eqs. (272) and (273)].

Projecting Eq. (276) against the basis

$$
\langle\mathcal{B}|=\left\{\left\langle 0_{0}^{\mathrm{CI}}\right|,\left\langle\mu_{k}^{\mathrm{CI}}\right|\right\}
$$

gives

$$
\begin{gathered}
\left\langle 0_{0}^{\mathrm{CI}}\left|H_{0}\right| 0_{0}^{\mathrm{CI}}\right\rangle=E_{0}^{\mathrm{CI}}, \\
\left\langle\mu_{k}^{\mathrm{CI}}\left|H_{0}\right| 0_{0}^{\mathrm{CI}}\right\rangle=0 .
\end{gathered}
$$

It follows from Eq. (279b) and the Hermiticity of the Hamiltonian that

$$
\left\langle 0_{0}^{\mathrm{CI}}\left|H_{0}\right| \mu_{k}^{\mathrm{CI}}\right\rangle=0
$$

is also satisfied.

Equations (279) and (280) describe the optimization conditions obtained when the variational principle is invoked to solve the time-independent Schrödinger equation [Eq. (87b)]. The zero-order Lagrangian multipliers in Eq. (194) satisfy Eq. (196), which in the CI model become

$$
\begin{aligned}
& \left\langle 0_{0}^{\mathrm{CI}}\left|\left[H_{0},\left|\mu_{k}^{\mathrm{CI}}\right\rangle\left\langle 0_{0}^{\mathrm{CI}}\right|\right]\right| 0_{0}^{\mathrm{CI}}\right\rangle \\
& \quad+\sum_{v_{m}} \bar{s}_{v_{m}}^{(0)}\left\langle v_{m}^{\mathrm{CI}}\left|\left[H_{0},\left|\mu_{k}^{\mathrm{CI}}\right\rangle\left\langle 0_{0}^{\mathrm{CI}}\right|\right]\right| 0_{0}^{\mathrm{CI}}\right\rangle=0,
\end{aligned}
$$

which may be written as

$\left\langle 0_{0}^{\mathrm{CI}}\left|H_{0}\right| \mu_{k}^{\mathrm{CI}}\right\rangle+\sum_{v_{m}} \bar{s}_{v_{m}}^{(0)}\left(\left\langle v_{m}^{\mathrm{CI}}\left|H_{0}\right| \mu_{k}^{\mathrm{CI}}\right\rangle-{ }^{\mathrm{CI}} E_{0} \delta_{\mu_{k} v_{m}}\right)=0$.

Applying Eq. (280) in Eq. (282), we thus see that the zeroorder multipliers vanish in CI theory,

$$
\bar{s}_{\mu_{k}}^{(0)}=0 .
$$

As $S_{0}=B_{0}$ is zero and

$$
S(t)=\sum_{\mu_{k}} s_{\mu_{k}}(t)\left|\mu_{k}^{\mathrm{CI}}\right\rangle\left\langle 0_{0}^{\mathrm{CI}}\right|,
$$

the time-dependent CI wave function [Eq. (252)] is linearly expanded around the unperturbed ground state $\left|0_{0}^{\mathrm{CI}}\right\rangle$,

$$
\left|0_{I}^{\mathrm{CI}}\right\rangle=\exp (S(t))\left|0_{0}^{\mathrm{CI}}\right\rangle=\left|0_{0}^{\mathrm{CI}}\right\rangle+\sum_{\mu_{k}} s_{\mu_{k}}(t)\left|\mu_{k}^{\mathrm{CI}}\right\rangle .
$$

We now derive explicit expressions for the CI molecular response functions through fourth order. To do this, we initially note that the expressions for ${ }^{c} \mathcal{L}_{\mathcal{H}}$ [Eq. (273)] and ${ }^{c} \mathcal{L}_{X_{j_{1}}}$ [Eq. (272)] in the CI model may be further simplified by replacing $H_{0}^{B_{0}}$ and $X_{j_{1}}^{B_{0}}$ with the bare operators and setting the zero-order multipliers to zero [Eq. (283)]. Using these simplifications and introducing the relabeling $|\mathscr{R}\rangle=\left|0_{0}^{\mathrm{CI}}\right\rangle$, $|k\rangle=\left|\mu_{k}^{\mathrm{CI}}\right\rangle, b_{k}=s_{\mu_{k}}, \bar{b}_{k}^{(\backslash 0)}=\bar{s}_{\mu_{k}}^{(\backslash 0)}$, the expression for ${ }^{c} \mathcal{L}_{\mathcal{H}}$ [Eq. (273)] becomes in the CI model

$$
\begin{aligned}
{ }^{c} \mathcal{L}_{\mathcal{H}}^{\mathrm{CI}}= & { }^{\mathrm{CI}} E_{0}+\sum_{\substack{\mu_{k} v_{m} \\
K M}} \bar{s}_{\mu_{k}}^{(\backslash 0)}\left(\omega_{K}\right) s_{v_{m}}\left(\omega_{M}\right) \\
& \times\left({ }^{\mathrm{CI}} J_{\mu_{k} v_{m}}-\omega_{M} \delta_{\mu_{k} v_{m}}\right) \Delta\left(\omega_{K}+\omega_{M}\right),
\end{aligned}
$$

where the CI Jacobian is given by [see Eq. (261)]

$$
\begin{aligned}
{ }^{\mathrm{CI}} J_{\mu_{k} v_{m}} & =\left\langle\mu_{k}^{\mathrm{CI}}\left|\left[H_{0},\left|v_{m}^{\mathrm{CI}}\right\rangle\left\langle 0_{0}^{\mathrm{CI}}\right|\right]\right| 0_{0}^{\mathrm{CI}}\right\rangle \\
& =\left\langle\mu_{k}^{\mathrm{CI}}\left|H_{0}\right| v_{m}^{\mathrm{CI}}\right\rangle-{ }^{\mathrm{CI}} E_{0} \delta_{\mu_{k} v_{m}} .
\end{aligned}
$$

We have used in Eq. (286) that ${ }^{c} \mathcal{L}_{H_{0}}^{N L}$ [Eq. (270a)] vanishes since Eq. (280) is satisfied. ${ }^{c} \mathcal{L}_{X_{j_{1}}}$ [Eq. (272)] becomes

$$
\begin{aligned}
{ }^{c} \mathcal{L}_{X_{j_{1}}}^{\mathrm{CI}}= & \left\langle 0_{0}^{\mathrm{CI}}\left|X_{j_{1}}\right| 0_{0}^{\mathrm{CI}}\right\rangle \Delta\left(\omega_{X_{j_{1}}}\right)+\sum_{\substack{v_{m} \\
v_{m}}} s_{v_{m}}\left(\omega_{M}\right)\left\langle 0_{0}^{\mathrm{CI}}\right| \\
& \times\left[X_{j_{1}},\left|v_{m}^{\mathrm{CI}}\right\rangle\left\langle 0_{0}^{\mathrm{CI}}\right|\right]\left|0_{0}^{\mathrm{CI}}\right\rangle \Delta\left(\omega_{X_{j_{1}}}+\omega_{M}\right) \\
& +\sum_{\mu_{k}} \bar{s}_{\mu_{k}}^{(\backslash 0)}\left(\omega_{K}\right)\left\langle\mu_{k}^{\mathrm{CI}}\left|X_{j_{1}}\right| 0_{0}^{\mathrm{CI}}\right\rangle \Delta\left(\omega_{X_{j_{1}}}+\omega_{K}\right) \\
& +\sum_{\mu_{k} v_{m}} \bar{s}_{\mu_{k}}^{(\backslash 0)}\left(\omega_{K}\right) s_{v_{m}}\left(\omega_{M}\right)\left\langle\mu_{k}^{\mathrm{CI}}\right|\left[X_{j_{1}},\left|v_{m}^{\mathrm{CI}}\right\rangle\left\langle 0_{0}^{\mathrm{CI}}\right|\right] \\
& \times\left|0_{0}^{\mathrm{CI}}\right\rangle \Delta\left(\omega_{X_{j_{1}}}+\omega_{K}+\omega_{M}\right) \\
& +\frac{1}{2} \sum_{\mu_{k_{k}}{ }^{v} \lambda_{n}} \bar{s}_{\mu_{k}}^{(\backslash 0)}\left(\omega_{K}\right) s_{v_{m}}\left(\omega_{M}\right) s_{\lambda_{n}}\left(\omega_{N}\right)\left\langle\mu_{k}^{\mathrm{CI}}\right| \\
& \times\left[\left[X_{j_{1}},\left|v_{m}^{\mathrm{CI}}\right\rangle\left\langle 0_{0}^{\mathrm{CI}}\right|\right],\left|\lambda_{n}^{\mathrm{CI}}\right\rangle\left\langle 0_{0}^{\mathrm{CI}}\right|\right]\left|0_{0}^{\mathrm{CI}}\right\rangle \\
& \times \Delta\left(\omega_{X_{j_{1}}}+\omega_{K}+\omega_{M}+\omega_{N}\right) .
\end{aligned}
$$

Expanding the commutators in Eq. (288), we obtain

$$
\begin{aligned}
{ }^{c} \mathcal{L}_{X_{j_{1}}}^{\mathrm{CI}}= & \left\langle 0_{0}^{\mathrm{CI}}\left|X_{j_{1}}\right| 0_{0}^{\mathrm{CI}}\right\rangle \Delta\left(\omega_{X_{j_{1}}}\right)+\sum_{\substack{v_{m} \\
v_{v_{m}}}}\left(\omega_{M}\right) \\
& \times\left\langle 0_{0}^{\mathrm{CI}}\left|X_{j_{1}}\right| \nu_{m}^{\mathrm{CI}}\right\rangle \Delta\left(\omega_{X_{j_{1}}}+\omega_{M}\right) \\
& +\sum_{\mu_{k}} \bar{s}_{\mu_{k}}^{(\backslash 0)}\left(\omega_{K}\right)\left\langle\mu_{k}^{\mathrm{CI}}\left|X_{j_{1}}\right| 0_{0}^{\mathrm{CI}}\right\rangle \Delta\left(\omega_{X_{j_{1}}}+\omega_{K}\right) \\
& +\sum_{\mu_{k} v_{m}} \bar{s}_{\mu_{k}}^{(\backslash 0)}\left(\omega_{K}\right) s_{v_{m}}\left(\omega_{M}\right) \\
& \times\left(\left\langle\mu_{k}^{\mathrm{CI}}\left|X_{j_{1}}\right| v_{m}^{\mathrm{CI}}\right\rangle-\delta_{\mu_{k} v_{m}}\left\langle 0_{0}^{\mathrm{CI}}\left|X_{j_{1}}\right| 0_{0}^{\mathrm{CI}}\right\rangle\right)
\end{aligned}
$$




$$
\begin{aligned}
& \times \Delta\left(\omega_{X_{j_{1}}}+\omega_{K}+\omega_{M}\right)-\left(\sum_{\substack{\mu_{k} \\
K L}} \bar{s}_{\mu_{k}}^{(\backslash 0)}\left(\omega_{K}\right) s_{\mu_{k}}\left(\omega_{L}\right)\right) \\
& \times \sum_{\substack{v_{m} \\
M}} s_{v_{m}}\left(\omega_{M}\right)\left\langle 0_{0}^{\mathrm{CI}}\left|X_{j_{1}}\right| v_{m}^{\mathrm{CI}}\right\rangle \\
& \times \Delta\left(\omega_{X_{j_{1}}}+\omega_{K}+\omega_{L}+\omega_{M}\right) .
\end{aligned}
$$

Using ${ }^{c} \mathcal{L}_{\mathcal{H}}$ [Eq. (286)] and ${ }^{c} \mathcal{L}_{X_{j_{1}}}$ [Eq. (289)], we follow the same route as in Sec. III $\mathrm{C}$ to derive response function expressions. We thus initially identify ${ }^{c} \mathcal{L}^{X_{j_{1}} \ldots X_{j_{n}}}\left(\omega_{X_{j_{1}}}, \ldots\right.$, $\omega_{X_{j n}}$ ) by writing ${ }^{c} \mathcal{L}^{(n)}$ in terms of the individual contributions from ${ }^{c} \mathcal{L}_{\mathcal{H}}^{\mathrm{CI}}$ [Eq. (286)] and ${ }^{c} \mathcal{L}_{X_{j_{1}}}^{\mathrm{CI}}$ [Eq. (289)],

$$
{ }^{c} \mathcal{L}^{(n)}={ }^{c} \mathcal{L}_{\mathcal{H}}^{(n)}+\sum_{j_{1}}{ }^{c} \mathcal{L}_{X_{j_{1}}}^{(n-1)} \epsilon_{X_{j_{1}}}\left(\omega_{X_{j_{1}}}\right),
$$

identifying, according to Eq. (244), the individual distinct contributions to $\mathcal{L}^{X_{j_{1}} \ldots X_{j_{n}}}\left(\omega_{X_{j_{1}}}, \ldots, \omega_{X_{j_{n}}}\right)$. We then obtain response equations by differentiating ${ }^{\mathcal{L} X_{j_{1}} \ldots X_{j_{n}}}\left(\omega_{X_{j_{1}}}\right.$, $\left.\ldots, \omega_{X_{j n}}\right) \quad$ with respect to $s_{\mu_{k}}^{X_{j_{1}} \cdots X_{j_{m}}}\left(\omega_{X_{j_{1}}}, \ldots\right.$, $\left.\omega_{X_{j_{m}}}\right)$ and $\bar{s}_{\mu_{k}}^{X_{j_{1}} \cdots X_{j_{m}}}\left(\omega_{X_{j_{1}}}, \ldots, \omega_{X_{j_{m}}}\right)$ using the stationary conditions of Eq. (245) and Eq. (246), respectively. Finally, we simplify ${ }^{c} \mathcal{L}^{X_{j_{1}} \ldots X_{j_{n}}}\left(\omega_{X_{j_{1}}}, \ldots, \omega_{X_{j_{n}}}\right)$ using the $2 n+1$ and $2 n+2$ rules (see, for example, Ref. 77) and obtain molecular response functions $\left\langle\left\langle X_{j_{1}} ; X_{j_{2}}, \ldots, X_{j_{n}}\right\rangle\right\rangle_{\omega_{X_{j_{2}}}, \ldots, \omega_{X_{j_{n}}}}$

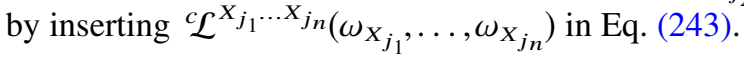

\section{B. Energy and first-order molecular properties}

From Eqs. (290) and (286), we determine ${ }^{c} \mathcal{L}^{(0)}$ as

$$
{ }^{c} \mathcal{L}^{(0)}={ }^{c} \mathcal{L}_{\mathcal{H}}^{(0)}={ }^{c} \mathcal{L}_{H_{0}}^{(0)}={ }^{\mathrm{CI}} E_{0}=\left\langle 0_{0}^{\mathrm{CI}}\left|H_{0}\right| 0_{0}^{\mathrm{CI}}\right\rangle .
$$

The first-order complex quasi-energy Lagrangian is obtained using Eqs. (286) and (289) as

$$
{ }^{c} \mathcal{L}^{X_{j_{1}}(0)}=\left\langle 0_{0}^{\mathrm{CI}}\left|X_{j_{1}}\right| 0_{0}^{\mathrm{CI}}\right\rangle,
$$

which, inserted in Eq. (243), gives the first-order molecular properties

$$
\left\langle\left\langle X_{j_{1}}\right\rangle\right\rangle_{0}=\frac{1}{2} C^{ \pm \omega}\left\langle 0_{0}^{\mathrm{CI}}\left|X_{j_{1}}\right| 0_{0}^{\mathrm{CI}}\right\rangle=\left\langle 0_{0}^{\mathrm{CI}}\left|X_{j_{1}}\right| 0_{0}^{\mathrm{CI}}\right\rangle .
$$

\section{Second-order molecular properties}

The second-order complex quasi-energy Lagrangian is obtained from Eq. (244) using Eqs. (286) and (289),

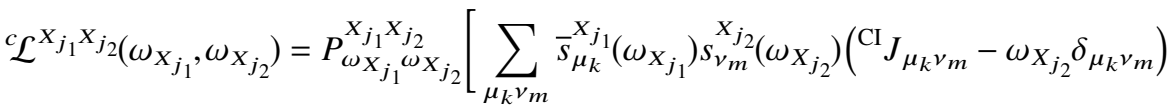

$$
\begin{aligned}
& \left.+\sum_{v_{m}} s_{v_{m}}^{X_{j_{2}}}\left(\omega_{X_{j_{2}}}\right)\left\langle 0_{0}^{\mathrm{CI}}\left|X_{j_{1}}\right| v_{m}^{\mathrm{CI}}\right\rangle+\sum_{\mu_{k}} \bar{s}_{\mu_{k}}^{X_{j_{2}}}\left(\omega_{X_{j_{2}}}\right)\left\langle\mu_{k}^{\mathrm{CI}}\left|X_{j_{1}}\right| 0_{0}^{\mathrm{CI}}\right\rangle\right], \quad \omega_{X_{j_{1}}}+\omega_{X_{j_{2}}}=0 .
\end{aligned}
$$

Applying the stationary condition of Eq. (246) to

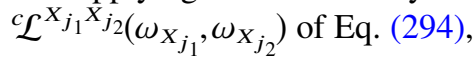

$$
0=\frac{\partial \mathcal{L}^{X_{j_{1}} X_{j_{2}}\left(\omega_{X_{j_{1}}}, \omega_{X_{j_{2}}}\right)}}{\partial \bar{s}_{\mu_{k}}^{X_{j_{2}}}\left(\omega_{X_{j_{2}}}\right)}
$$

gives

$$
\sum_{v_{m}}\left({ }^{\mathrm{CI}} J_{\mu_{k} v_{m}}-\omega_{X_{j_{1}}} \delta_{\mu_{k} v_{m}}\right) s_{v_{m}}^{X_{j_{1}}}\left(\omega_{X_{j_{1}}}\right)=-\left\langle\mu_{k}^{\mathrm{CI}}\left|X_{j_{1}}\right| 0_{0}^{\mathrm{CI}}\right\rangle .
$$

Equation (296) is the first-order response equation for the firstorder coefficients $s_{v_{m}}^{X_{j_{1}}}\left(\omega_{X_{j_{1}}}\right)$. Similarly, applying the stationary condition of Eq. (245) gives a response equation for the firstorder multipliers $\bar{s}_{v_{m}}^{X_{j_{1}}}\left(\omega_{X_{j_{1}}}\right)$,

$$
\sum_{v_{m}} \bar{s}_{v_{m}}^{X_{j_{1}}}\left(\omega_{X_{j_{1}}}\right)\left({ }^{\mathrm{CI}} J_{v_{m} \mu_{k}}+\omega_{X_{j_{1}}} \delta_{v_{m} \mu_{k}}\right)=-\left\langle 0_{0}^{\mathrm{CI}}\left|X_{j_{1}}\right| \mu_{k}^{\mathrm{CI}}\right\rangle,
$$

where for the left-hand side, we have used the condition $\omega_{X_{j_{1}}}+\omega_{X_{j_{2}}}=0$. It follows from Eqs. (296) and (297) that the first-order amplitudes and the first-order multipliers are the adjoints of each other,

$$
\bar{s}_{\mu_{k}}^{X_{j_{1}}}\left(\omega_{X_{j_{1}}}\right)=s_{\mu_{k}}^{X_{j_{1}}{ }^{*}}\left(-\omega_{X_{j_{1}}}\right) .
$$

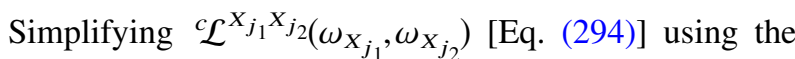
$2 n+1$ and $2 n+2$ rules $^{77}$ and inserting the simplified form

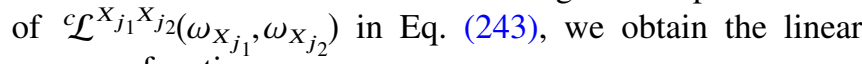
response function

$$
\begin{aligned}
\left\langle\left\langle X_{j_{1}} ; X_{j_{2}}\right\rangle\right\rangle_{\omega_{X_{j_{2}}}}= & \frac{1}{2} C^{ \pm \omega} P_{\omega_{X_{j_{1}}} \omega_{X_{j_{2}}} X_{v_{m}} \sum_{j_{2}}}{ }_{s_{v_{m}}}^{X_{j_{2}}}\left(\omega_{X_{j_{2}}}\right) \\
& \times\left\langle 0_{0}^{\mathrm{CI}}\left|X_{j_{1}}\right| v_{m}^{\mathrm{CI}}\right\rangle, \quad \omega_{X_{j_{1}}}+\omega_{X_{j_{2}}}=0,
\end{aligned}
$$

where $s_{v_{m}}^{X_{j_{2}}}\left(\omega_{X_{j_{2}}}\right)$ are obtained by solving Eq. (296).

\section{Third-order molecular properties}

Following the same route as for $\mathcal{L}^{X_{j_{1}} X_{j_{2}}}\left(\omega_{X_{j_{1}}}, \omega_{X_{j_{2}}}\right)$, we

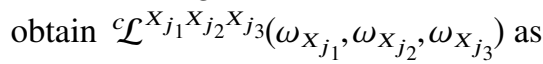




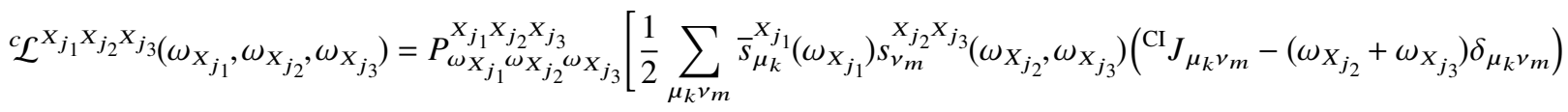

$$
\begin{aligned}
& +\frac{1}{2} \sum_{\mu_{k} v_{m}} \bar{s}_{\mu_{k}}^{X_{j_{1}} X_{j_{2}}}\left(\omega_{X_{j_{1}}}, \omega_{X_{j_{2}}}\right) s_{v_{m}}^{X_{j_{3}}}\left(\omega_{X_{j_{3}}}\right)\left({ }^{\mathrm{C}} J_{\mu_{k} v_{m}}-\omega_{X_{j_{3}}} \delta_{\mu_{k} v_{m}}\right) \\
& +\frac{1}{2} \sum_{v_{m}} s_{v_{m}}^{X_{j_{2}} X_{j_{3}}}\left(\omega_{X_{j_{2}}}, \omega_{X_{j_{3}}}\right)\left\langle 0_{0}^{\mathrm{CI}}\left|X_{j_{1}}\right| v_{m}^{\mathrm{CI}}\right\rangle+\frac{1}{2} \sum_{\mu_{k}} \bar{s}_{\mu_{k}}^{X_{j_{2}} X_{j_{3}}}\left(\omega_{X_{j_{2}}}, \omega_{X_{j_{3}}}\right)\left\langle\mu_{k}^{\mathrm{CI}}\left|X_{j_{1}}\right| 0_{0}^{\mathrm{CI}}\right\rangle \\
& \left.+\sum_{\mu_{k} v_{m}} \bar{s}_{\mu_{k}}^{X_{j_{2}}}\left(\omega_{X_{j_{2}}}\right) s_{v_{m}}^{X_{j_{3}}}\left(\omega_{X_{j_{3}}}\right)\left(\left\langle\mu_{k}^{\mathrm{CI}}\left|X_{j_{1}}\right| v_{m}^{\mathrm{CI}}\right\rangle-\left\langle 0_{0}^{\mathrm{CI}}\left|X_{j_{1}}\right| 0_{0}^{\mathrm{CI}}\right\rangle \delta_{\mu_{k} v_{m}}\right)\right], \quad \omega_{X_{j_{1}}}+\omega_{X_{j_{2}}}+\omega_{X_{j_{3}}}=0
\end{aligned}
$$

where the factor $\frac{1}{2}$ in the first four terms arises from Eq. (115).

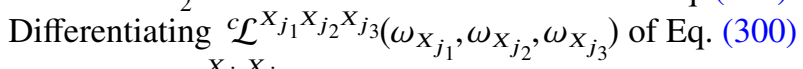
with respect to $\bar{s}_{\mu_{k}}^{X_{j_{2}} X_{j_{3}}}\left(\omega_{X_{j}}, \omega_{X_{j_{3}}}\right)$ and using the stationary condition of Eq. (246), we recover the first-order response equation of Eq. (296). Similarly, differentiating

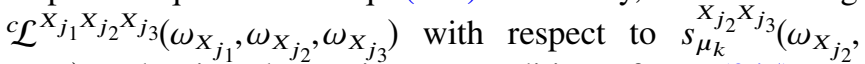
$\omega_{X_{j_{3}}}$ ) and using the stationary condition of Eq. (245), we recover the first-order multipliers equation of Eq. (297).

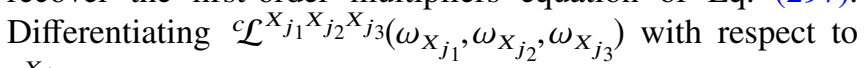
$\bar{s}_{\mu_{k}}^{X_{j_{3}}}\left(\omega_{X_{j_{3}}}\right)$ and using the stationary condition of Eq. (246) gives the response equation for the second-order coefficients,

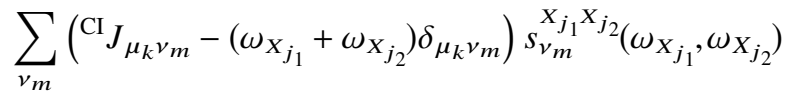

$$
\begin{aligned}
& =-P_{\omega_{X_{j_{1}}} \omega_{X_{j_{2}}}}^{X_{j_{j_{m}} X_{j_{2}}}} \sum_{v_{m}}\left(\left\langle\mu_{k}^{\mathrm{CI}}\left|X_{j_{1}}\right| v_{m}^{\mathrm{CI}}\right\rangle-\left\langle 0_{0}^{\mathrm{CI}}\left|X_{j_{1}}\right| 0_{0}^{\mathrm{CI}}\right\rangle \delta_{\mu_{k} v_{m}}\right) \\
& \times{ }_{v_{m}}^{X_{j_{2}}}\left(\omega_{X_{j_{2}}}\right),
\end{aligned}
$$

from which $s_{v_{m}}^{X_{j_{1}} X_{j_{2}}}\left(\omega_{X_{j_{1}}}, \omega_{X_{j_{2}}}\right)$, needed in Subsection VI E for the fourth-order properties, may be calculated.

Using the $2 n+1$ and $2 n+2$ rules, the third-order molecular property expression becomes

$$
\begin{aligned}
& \left\langle\left\langle X_{j_{1}} ; X_{j_{2}}, X_{j_{3}}\right\rangle\right\rangle_{\omega_{X_{j_{2}}}, \omega_{X_{j_{3}}}}
\end{aligned}
$$

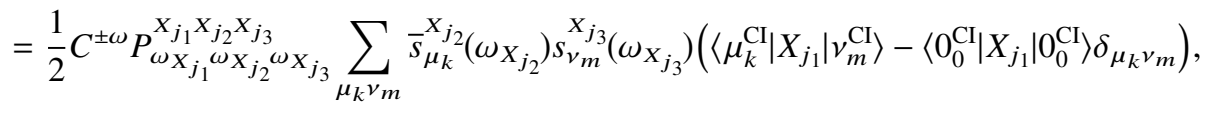

$$
\begin{aligned}
& \omega_{X_{j_{1}}}+\omega_{X_{j_{2}}}+\omega_{X_{j_{3}}}=0,
\end{aligned}
$$

where $s_{\mu_{k}}^{X_{j_{3}}}\left(\omega_{X_{j_{3}}}\right)$ and $\bar{s}_{\mu_{k}}^{X_{j_{2}}}\left(\omega_{X_{j_{2}}}\right)$ are obtained by solving Eqs. (296) and (297), respectively.

\section{E. Fourth-order molecular properties}

Using the $2 n+1$ and $2 n+2$ rules, we obtain fourth-order molecular properties in terms of the first- and second-order coefficients and first-order multipliers as

$$
\begin{aligned}
& \left\langle\left\langle X_{j_{1}} ; X_{j_{2}}, X_{j_{3}}, X_{j_{4}}\right\rangle\right\rangle_{\omega_{X_{j_{2}}}, \omega_{X_{j_{3}}}, \omega_{X_{j_{4}}}}
\end{aligned}
$$

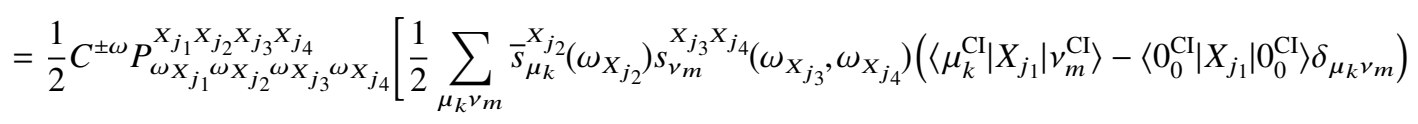

$$
\begin{aligned}
& \left.-\left(\sum_{\mu_{k}} \bar{s}_{\mu_{k}}^{X_{j_{2}}}\left(\omega_{X_{j_{2}}}\right) s_{\mu_{k}}^{X_{j_{3}}}\left(\omega_{X_{j_{3}}}\right)\right) \sum_{v_{m}}{ }_{s_{v_{m}}}^{X_{j_{4}}}\left(\omega_{X_{j_{4}}}\right)\left\langle 0_{0}^{\mathrm{CI}}\left|X_{j_{1}}\right| v_{m}^{\mathrm{CI}}\right\rangle\right], \quad \omega_{X_{j_{1}}}+\omega_{X_{j_{2}}}+\omega_{X_{j_{3}}}+\omega_{X_{j_{4}}}=0
\end{aligned}
$$


where $s_{\mu_{k}}^{X_{j_{3}}}\left(\omega_{X_{j_{3}}}\right), \quad \bar{s}_{\mu_{k}}^{X_{j_{2}}}\left(\omega_{X_{j_{2}}}\right)$, and $s_{\mu_{k}}^{X_{j_{3}} X_{j_{4}}}\left(\omega_{X_{j_{3}}}, \omega_{X_{j_{4}}}\right)$ are obtained by solving Eqs. (296), (297), and (301), respectively.

\section{F. The $\mathrm{Cl}$ Jacobian as a part of the $\mathrm{Cl}$ eigenvalue equation}

We now consider a matrix representation of the Hermitian eigenvalue equation for the unperturbed Hamiltonian in the basis of Eq. (275). We then show that the Jacobian matrix contains a sub-matrix of the Hamiltonian matrix.

\section{The $\mathrm{Cl}$ eigenvalue equation}

The time development of the CI wave function in Eq. (285) is described in terms of a linear expansion in the basis $|\mathcal{B}\rangle$ of Eq. (275). We now consider a matrix representation of the Hermitian eigenvalue equation for the unperturbed Hamiltonian $H_{0}$ in this basis

$$
{ }^{\mathrm{C}} \mathbf{H}_{0}{ }^{\mathrm{Cl}} \widetilde{\mathbf{C}}={ }^{\mathrm{Cl}} \widetilde{\mathbf{C}}{ }^{\mathrm{Cl}} \mathbf{E} \text {. }
$$

The unperturbed Hamiltonian matrix, ${ }^{\mathrm{C}} \mathbf{H}_{0}$, has the block structure

$$
{ }^{\mathrm{CI}} \mathbf{H}_{0}=\left(\begin{array}{cc}
{ }^{\mathrm{CI}} E_{0} & \mathbf{0} \\
\mathbf{0} & { }^{\mathrm{CI}} \mathbf{H}_{0}^{\perp}
\end{array}\right),
$$

where ${ }^{\mathrm{C}} \mathbf{H}_{0}^{\perp}$ denotes the sub-block of the Hamiltonian matrix in the orthogonal complement subspace $\left\{\left|\mu_{k}^{\mathrm{CI}}\right\rangle\right\}$,

$$
\left[{ }^{\mathrm{CI}} \mathbf{H}_{0}^{\perp}\right]_{\mu_{k} v_{m}}=\left[{ }^{\mathrm{CI}} \mathbf{H}_{0}\right]_{\mu_{k} v_{m}}=\left\langle\mu_{k}^{\mathrm{CI}}\left|H_{0}\right| v_{m}^{\mathrm{CI}}\right\rangle .
$$

To obtain the block structure in Eq. (305), we have used Eqs. (279) and (280),

$$
\begin{gathered}
{\left[{ }^{\mathrm{CI}} \mathbf{H}_{0}\right]_{00}=\left\langle 0_{0}^{\mathrm{CI}}\left|H_{0}\right| 0_{0}^{\mathrm{CI}}\right\rangle={ }^{\mathrm{CI}} E_{0},} \\
{\left[{ }^{\mathrm{C}} \mathbf{H}_{0}\right]_{0 \mu_{k}}=\left\langle 0_{0}^{\mathrm{CI}}\left|H_{0}\right| \mu_{k}^{\mathrm{CI}}\right\rangle=0,} \\
{\left[{ }^{\mathrm{Cl}} \mathbf{H}_{0}\right]_{\mu_{k} 0}=\left\langle\mu_{k}^{\mathrm{CI}}\left|H_{0}\right| 0_{0}^{\mathrm{CI}}\right\rangle=0 .}
\end{gathered}
$$

The ${ }^{\mathrm{Cl}} \mathbf{E}$ matrix is diagonal and contains the energies of the unperturbed Hamiltonian $H_{0}$,

$$
{ }^{\mathrm{C}} \mathbf{E}=\left(\begin{array}{cccc}
{ }^{\mathrm{CI}} E_{0} & & & \\
& { }^{\mathrm{CI}} E_{1} & & \\
& & { }^{\mathrm{CI}} E_{2} & \\
& & & \ddots
\end{array}\right) .
$$

The block structure of Eq. (305) implies that

$$
{ }^{\mathrm{C}} \widetilde{\mathbf{C}}=\left(\begin{array}{cc}
1 & \mathbf{0} \\
\mathbf{0} & { }^{\mathrm{CI}} \mathbf{C}
\end{array}\right) .
$$

The ground state vector therefore becomes

$$
{ }^{\mathrm{C}} \widetilde{\mathbf{C}}_{0}=\left(\begin{array}{l}
1 \\
\mathbf{0}
\end{array}\right) \text {, }
$$

whereas excited state vectors for energies ${ }^{\mathrm{Cl}} E_{n}, n=1,2, \ldots$, become

$$
{ }^{\mathrm{C}} \widetilde{\mathbf{C}}_{n}=\left(\begin{array}{c}
0 \\
{ }^{\mathrm{CI}} \mathbf{C}_{n}
\end{array}\right),
$$

where ${ }^{\mathrm{Cl}} \mathbf{C}_{n}$ is the $n$th column of the matrix ${ }^{\mathrm{Cl}} \mathbf{C}$ [Eq. (311)]. As the matrix ${ }^{\mathrm{Cl}} \mathbf{H}_{0}$ is Hermitian, the eigenvectors may be chosen to constitute an orthonormal set

$$
{ }^{\mathrm{CI}} \mathbf{C}^{\dagger} \mathrm{CI} \mathbf{C}=\mathbf{I} \text {. }
$$

For later convenience, we express the eigenstates in the bracket form as

$$
\begin{aligned}
& \left|0_{\mathrm{R}}^{\mathrm{CI}}\right\rangle=\left\{\left|0_{0}^{\mathrm{CI}}\right\rangle,\left|0_{1}^{\mathrm{CI}}\right\rangle,\left|0_{2}^{\mathrm{CI}}\right\rangle, \cdots\right\}, \\
& \left\langle 0_{\mathrm{L}}^{\mathrm{CI}}\right|=\left\{\left\langle 0_{0}^{\mathrm{CI}}\right|,\left\langle 0_{1}^{\mathrm{CI}}\right|,\left\langle 0_{2}^{\mathrm{CI}}\right|, \cdots\right\},
\end{aligned}
$$

where, for $n \neq 0$,

$$
\left|0_{n}^{\mathrm{CI}}\right\rangle=\sum_{\mu_{k}}{ }^{\mathrm{CI}} C_{\mu_{k} n}\left|\mu_{k}^{\mathrm{CI}}\right\rangle
$$

and where ${ }^{\mathrm{CI}} C_{\mu_{k} n}$ are elements of the ${ }^{\mathrm{CI}} \mathbf{C}_{n}$ vector [Eq. (313)].

\section{The $\mathrm{Cl}$ Jacobian eigenvalue equation}

We now consider the CI Jacobian in more detail. It follows from Eqs. (287) and (306) that the Jacobian matrix may be expressed in terms of a matrix representation of $H_{0}$ in the orthogonal complement subspace $\left\{\left|\mu_{k}^{\mathrm{CI}}\right\rangle\right\}$ minus a diagonal matrix containing ${ }^{\mathrm{CI}} E_{0}$,

$$
{ }^{\mathrm{CI}} \mathbf{J}={ }^{\mathrm{Cl}} \mathbf{H}_{0}^{\perp}-{ }^{\mathrm{CI}} E_{0} \mathbf{I} .
$$

Using the block structure of ${ }^{\mathrm{Cl}} \mathbf{H}_{0}$ [Eq. (305)] and ${ }^{\mathrm{Cl}} \widetilde{\mathbf{C}}$ [Eq. (311)] and denoting the sub-block of ${ }^{\mathrm{Cl}} \mathbf{E}$ [Eq. (310)] in the orthogonal complement subspace as ${ }^{\mathrm{Cl}} \mathbf{E}^{\perp}$, the eigenvalue equation [Eq. (304)] in the orthogonal complement subspace may be written as

$$
\left({ }^{\mathrm{Cl}} \mathbf{H}_{0}^{\perp}-{ }^{\mathrm{Cl}} E_{0} \mathbf{I}\right){ }^{\mathrm{Cl}} \mathbf{C}={ }^{\mathrm{Cl}} \mathbf{C}\left({ }^{\mathrm{Cl}} \mathbf{E}^{\perp}-{ }^{\mathrm{Cl}} E_{0} \mathbf{I}\right),
$$

or, equivalently, as

$$
{ }^{\mathrm{CI}} \mathbf{J}{ }^{\mathrm{Cl}} \mathbf{C}={ }^{\mathrm{CI}} \mathbf{C} \Delta \boldsymbol{\Delta}^{\mathrm{Cl}} \mathbf{E},
$$

where

$\Delta^{\mathrm{CI}} \mathbf{E}={ }^{\mathrm{CI}} \mathbf{E}^{\perp}-{ }^{\mathrm{CI}} E_{0} \mathbf{I}=\left(\begin{array}{llll}{ }^{\mathrm{CI}} E_{10} & & & \\ & { }^{\mathrm{CI}} E_{20} & & \\ & & { }^{\mathrm{Cl}} E_{30} & \\ & & & \ddots\end{array}\right)$

and

$$
{ }^{\mathrm{CI}} E_{n 0}={ }^{\mathrm{CI}} E_{n}-{ }^{\mathrm{CI}} E_{0}, \quad n \neq 0 .
$$

Equations (320)-(322) show that the eigenvalues of ${ }^{\mathrm{CI}} \mathbf{J}$ are equal to the excitation energies of the unperturbed system.

From Eqs. (319) and (311), we see that the eigenvectors of the CI Jacobian, ${ }^{\mathrm{CI}} \mathbf{J}$, are equal to the excited state eigenvectors of the CI Hamiltonian matrix, ${ }^{\mathrm{Cl}} \mathbf{H}_{0}^{\perp}$, in Eq. (305) 
in accordance with Eq. (128). The excited states are thus linearly parametrized in the basis $|\mathcal{B}\rangle$ of Eq. (275) as is the time evolution of the ground state [Eq. (285)]. As there is no interaction between the ground state and the orthogonal complement set of states, neither for the unperturbed system nor for the time-evolving system, we therefore have an explicit representation of the excited states in CI response theory.

\section{G. Comparison of response functions obtained from the quasi-energy and from the quasi-energy Lagrangian}

In Secs. III and VI, molecular response functions were determined using a linearly parametrized unperturbed ground state and employing a linear parametrization of the time evolution of the perturbed wave function. In Sec. III, the Hermitian eigenvalue equation for the time evolution [Eq. (32)] was solved using the variational principle, while in Sec. VI, it was solved via projection.

When the Hermitian eigenvalue equation was solved using the variational principle, the molecular response properties were determined as derivatives of the quasi-energy $\mathcal{E}$ [Eq. (133)] given in terms of $\mathcal{E}_{\mathcal{H}}$ [Eq. (131)] and $\mathcal{E}_{X_{j_{1}}}$ [Eq. (132)], where each of these contributions contains terms to infinite order introduced by a normalization constant $N$ through Eq. (95) [cf. Eqs. (98)-(100)].

By contrast, when the Hermitian eigenvalue equation was solved via projection, the molecular response properties were obtained as derivatives of the complex quasienergy Lagrangian ${ }^{c} \mathcal{L}^{\mathrm{CI}}$ [Eq. (274)] given in terms of ${ }^{c} \mathcal{L}_{\mathcal{H}}^{\mathrm{CI}}$ [Eq. (286)] and ${ }^{c} \mathcal{L}_{X_{j_{1}}}^{\mathrm{CI}}$ [Eq. (289)]. The two definitions lead to the same response properties, see Eq. (239). However, the ${ }^{c} \mathcal{L}_{\mathcal{H}}^{\mathrm{CI}}$ [Eq. (286)] and ${ }^{c} \mathcal{L}_{X_{j_{1}}}^{\mathrm{CI}}$ [Eq. (289)] contributions do not contain a normalization constant. ${ }^{c} \mathcal{L}^{\mathrm{CI}}$ therefore has a simpler structure than $\mathcal{E}$. Although ${ }^{c} \mathcal{L}^{\mathrm{CI}}$ contains an extra set of parameters compared to $\mathcal{E}$, the Lagrangian multipliers, these extra parameters are through the $n$th order easily obtained from the $n$ th-order wave function amplitudes. In particular, the first-order multipliers are simply the adjoints of the first-order wave function amplitudes [Eq. (298)].

The complex quasi-energy Lagrangian [Eq. (274)] does not contain the norm since the time development is expressed in terms of an intermediate normalized state [Eq. (89a)] rather than in terms of the unit-normalized regular wave function [Eq. (88)]. This simplification is similar to the one obtained in time-independent perturbation theory, where the use of intermediate normalization also simplifies the determination of an order expansion for the energy and the wave function, removing the reference to the normalization constant.

We have seen in this section the advantages of solving the Hermitian eigenvalue equation via projection for a linear parametrization of the time evolution of the perturbed wave function. However, for non-variational wave function models, the Hermitian eigenvalue equation [Eq. (32)] can only be solved via projection and molecular response properties then have to be determined using a quasi-energy Lagrangian as described in Sec. IV. In Secs. VII and VIII, we describe the determination of response functions, where the unperturbed ground state is a coupled cluster state. In Sec. VII, the time evolution is described in terms of a linear parametrization, while in Sec. VIII, the time evolution is described in terms of an exponential parametrization.

\section{MOLECULAR PROPERTIES FOR THE CC-CI MODEL}

In Sec. VI, we have derived molecular response functions using the CI model, where both the time-independent unperturbed state and the time development are linearly parametrized. In this and Sec. VIII, we consider an approach where the time-independent unperturbed state is an exponentially parametrized coupled cluster state. In this section, we consider the CC-CI model [Eq. (254)], where the exponential parametrization of the unperturbed state is followed by a linear parametrization of the time development, whereas in Sec. VIII, we turn our attention to the CC model [Eq. (253)], where the time development is also exponentially parametrized.

\section{A. Parametrization of the $\mathrm{CC}-\mathrm{Cl}$ model}

In the CC-CI model [Eq. (254)], the set $|\mathcal{B}\rangle$ becomes

$$
|\mathcal{B}\rangle=\left\{|\mathrm{HF}\rangle,\left|\mu_{k}^{\mathrm{CC}}\right\rangle\right\},
$$

where $|\mathrm{HF}\rangle$ is the Hartree-Fock reference state and the orthogonal complement set of states $\left|\mu_{k}^{\mathrm{CC}}\right\rangle$ is defined via Eq. (250).

For the unperturbed system, the coupled cluster energy reads [Eq. (193a)]

$$
{ }^{\mathrm{CC}} E_{0}=\left\langle\mathrm{HF}\left|H_{0}^{T_{0}}\right| \mathrm{HF}\right\rangle,
$$

where the cluster amplitudes, $t_{\mu_{k}}^{(0)}$, satisfy the coupled cluster amplitudes equation (cf. Eq. (193b))

$$
\left\langle\mu_{k}^{\mathrm{CC}}\left|H_{0}^{T_{0}}\right| \mathrm{HF}\right\rangle=0 .
$$

The coupled cluster zero-order Lagrangian (cf. Eq. (194)) therefore reads

$$
L_{0}=\left\langle\mathrm{HF}\left|H_{0}^{T_{0}}\right| \mathrm{HF}\right\rangle+\sum_{\mu_{k}} \bar{t}_{\mu_{k}}^{(0)}\left\langle\mu_{k}^{\mathrm{CC}}\left|H_{0}^{T_{0}}\right| \mathrm{HF}\right\rangle .
$$

The zero-order Lagrangian multipliers satisfy (cf. Eq. (196))

$$
\sum_{\mu_{k}} \bar{t}_{\mu_{k}}^{(0)}{ }^{\mathrm{CC}} J_{\mu_{k} v_{m}}=-\eta_{v_{m}}
$$

where, using Eq. (261), we have introduced the CC Jacobian

$$
\begin{aligned}
{ }^{\mathrm{CC}} J_{\mu_{k} v_{m}} & =\left\langle\mu_{k}^{\mathrm{CC}}\left|\left[H_{0}^{T_{0}}, \tau_{v_{m}}\right]\right| \mathrm{HF}\right\rangle \\
& =\left\langle\mu_{k}^{\mathrm{CC}}\left|H_{0}^{T_{0}}\right| v_{m}^{\mathrm{CC}}\right\rangle-\delta_{\mu_{k} v_{m}}\left\langle\mathrm{HF}\left|H_{0}^{T_{0}}\right| \mathrm{HF}\right\rangle
\end{aligned}
$$

and where

$$
\eta_{v_{m}}=\left\langle\mathrm{HF}\left|\left[H_{0}^{T_{0}}, \tau_{v_{m}}\right]\right| \mathrm{HF}\right\rangle=\left\langle\mathrm{HF}\left|H_{0}^{T_{0}}\right| v_{m}^{\mathrm{CC}}\right\rangle .
$$

Using that in the CC-CI model, the $B(t)$ operator has the form

$$
S(t)=\sum_{\mu_{k}} s_{\mu_{k}}(t)\left|\mu_{k}^{\mathrm{CC}}\right\rangle\langle\mathrm{HF}|
$$


the time-dependent CC-CI wave function $\left|0_{I}^{\mathrm{CC}-\mathrm{CI}}\right\rangle$ [Eq. (254)] may be written as

$$
\begin{aligned}
\left|0_{I}^{\mathrm{CC}-\mathrm{CI}}\right\rangle & =\exp \left(T_{0}\right) \exp (S(t))|\mathrm{HF}\rangle \\
& =\exp \left(T_{0}\right)|\mathrm{HF}\rangle+\sum_{\mu_{k}} s_{\mu_{k}}(t) \exp \left(T_{0}\right)\left|\mu_{k}^{\mathrm{CC}}\right\rangle
\end{aligned}
$$

Equation (331) shows that the coupled cluster state $\exp \left(T_{0}\right)|\mathrm{HF}\rangle$ is obtained for the unperturbed system in the CC-CI model and that the perturbation expansion of the quasi-energy Lagrangian therefore may be carried out with the unperturbed coupled cluster state as expansion point. Eq. (331) further shows that in the CC-CI model the perturbation-induced time evolution is expressed in terms of a linear CI expansion in the basis of the many-electron functions

$$
\left|\mathcal{B}^{T_{0}}\right\rangle=\left\{\exp \left(T_{0}\right)|\mathrm{HF}\rangle, \exp \left(T_{0}\right)\left|\mu_{k}^{\mathrm{CC}}\right\rangle\right\} .
$$

The CC-CI molecular response functions may be obtained following the same route as for the CI model in Sec. VI and using the following relabeling in ${ }^{c} \mathcal{L}_{\mathcal{H}}$ [Eq. (273)] and ${ }^{c} \mathcal{L}_{X_{j_{1}}}$ [Eq. (272)]: $|\mathscr{R}\rangle=|\mathrm{HF}\rangle,|k\rangle=\left|\mu_{k}^{\mathrm{CC}}\right\rangle, B_{0}=T_{0}, \beta_{k}$ $=\left|\mu_{k}^{\mathrm{CC}}\right\rangle\langle\mathrm{HF}|, b_{k}^{(0)}=t_{\mu_{k}}^{(0)}, \bar{b}_{k}^{(0)}=\bar{t}_{\mu_{k}}^{(0)}, b_{k}=s_{\mu_{k}}$, and $\bar{b}_{k}=\bar{s}_{\mu_{k}}$. In the following, we determine the CC-CI response functions through fourth order.

\section{B. Energy and first-order molecular properties}

From Eq. (273), we determine ${ }^{c} \mathcal{L}^{(0)}$ as

$$
{ }^{c} \mathcal{L}^{(0)}={ }^{c} \mathcal{L}_{\mathcal{H}}^{(0)}={ }^{c} \mathcal{L}_{H_{0}}^{(0)}={ }^{\mathrm{CC}} E_{0}=\left\langle\mathrm{HF}\left|H_{0}^{T_{0}}\right| \mathrm{HF}\right\rangle
$$

since the expansion coefficients $s_{k}\left(\omega_{K}\right)$ do not contain zeroorder contributions.

The first-order complex quasi-energy Lagrangian is obtained using Eqs. (272) and (273) as

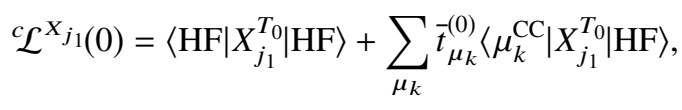

which, inserted in Eq. (243), gives the first-order molecular properties

$\left\langle\left\langle X_{j_{1}}\right\rangle\right\rangle_{0}=\frac{1}{2} C^{ \pm \omega}\left(\left\langle\mathrm{HF}\left|X_{j_{1}}^{T_{0}}\right| \mathrm{HF}\right\rangle+\sum_{\mu_{k}} \bar{t}_{\mu_{k}}^{(0)}\left\langle\mu_{k}^{\mathrm{CC}}\left|X_{j_{1}}^{T_{0}}\right| \mathrm{HF}\right\rangle\right)$,

where the zero-order amplitudes, $t_{\mu_{k}}^{(0)}$, and the zero-order multipliers, $\bar{t}_{\mu_{k}}^{(0)}$, are obtained by solving Eqs. (325) and (327), respectively.

\section{Second-order molecular properties}

The second-order complex quasi-energy Lagrangian is obtained from Eqs. (272) and (273),

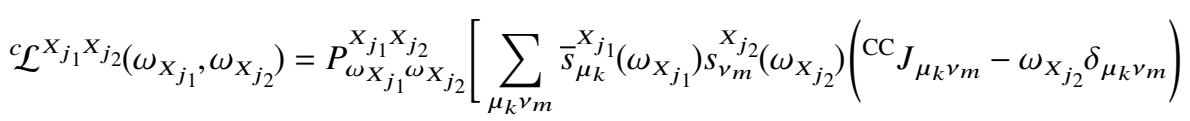

$$
\begin{aligned}
& -\sum_{\mu_{k}} \bar{t}_{\mu_{k}}^{(0)} s_{\mu_{k}}^{X_{j_{1}}}\left(\omega_{X_{j_{1}}}\right) \sum_{v_{m}}{ }_{s_{v_{m}}}^{X_{j_{2}}}\left(\omega_{X_{j_{2}}}\right)\left\langle\mathrm{HF}\left|H_{0}^{T_{0}}\right| v_{m}^{\mathrm{CC}}\right\rangle+\sum_{v_{m}}{ }_{s_{v_{m}}}^{X_{j_{2}}}\left(\omega_{X_{j_{2}}}\right)\left\langle\mathrm{HF}\left|\left[X_{j_{1}}^{T_{0}},\left|v_{m}^{\mathrm{CC}}\right\rangle\langle\mathrm{HF}|\right]\right| \mathrm{HF}\right\rangle \\
& \left.+\sum_{\mu_{k}} \bar{s}_{\mu_{k}}^{X_{j_{2}}}\left(\omega_{X_{j_{2}}}\right)\left\langle\mu_{k}^{\mathrm{CC}}\left|X_{j_{1}}^{T_{0}}\right| \mathrm{HF}\right\rangle+\sum_{\mu_{k} v_{m}} \bar{t}_{\mu_{k}}^{(0)}{ }_{v_{v_{m}}}^{X_{j_{2}}}\left(\omega_{X_{j_{2}}}\right)\left\langle\mu_{k}^{\mathrm{CC}}\left|\left[X_{j_{1}}^{T_{0}},\left|v_{m}^{\mathrm{CC}}\right\rangle\langle\mathrm{HF}|\right]\right| \mathrm{HF}\right\rangle\right], \quad \omega_{X_{j_{1}}}+\omega_{X_{j_{2}}}=0,
\end{aligned}
$$

where we have used that

$$
{ }^{\mathrm{CC}} J_{\mu_{k} v_{m}}={ }^{\mathrm{CC}-\mathrm{CI}} J_{\mu_{k} v_{m}}
$$

as seen from Eqs. (269) and (328).

Applying the stationary condition of Eq. (246) to

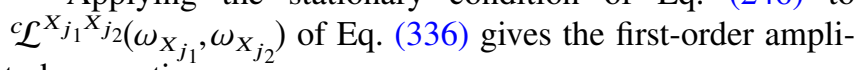
tudes equation

$$
\sum_{v_{m}}\left({ }^{\mathrm{CC}} J_{\mu_{k} v_{m}}-\omega_{X_{j_{1}}} \delta_{\mu_{k} v_{m}}\right) s_{v_{m}}^{X_{j_{1}}}\left(\omega_{X_{j_{1}}}\right)=-\left\langle\mu_{k}^{\mathrm{CC}}\left|X_{j_{1}}^{T_{0}}\right| \mathrm{HF}\right\rangle
$$

Similarly, applying the stationary condition of Eq. (245) gives the first-order multipliers equation

$$
\begin{aligned}
& \sum_{v_{m}} \bar{s}_{v_{m}}^{X_{j_{1}}}\left(\omega_{X_{j_{1}}}\right)\left({ }^{\mathrm{CC}} J_{v_{m} \mu_{k}}+\omega_{X_{j_{1}}} \delta_{v_{m} \mu_{k}}\right) \\
& =\left(\sum_{v_{m}} \bar{t}_{v_{m}}^{(0)}{ }_{s_{v_{m}}}^{X_{j_{1}}}\left(\omega_{X_{j_{1}}}\right)\right)\left\langle\mathrm{HF}\left|H_{0}^{T_{0}}\right| \mu_{k}^{\mathrm{CC}}\right\rangle \\
& +\bar{t}_{\mu_{k}}^{(0)} \sum_{v_{m}}{ }_{s_{v_{m}}}^{X_{j_{1}}}\left(\omega_{X_{j_{1}}}\right)\left\langle\mathrm{HF}\left|H_{0}^{T_{0}}\right| v_{m}^{\mathrm{CC}}\right\rangle \\
& -\left\langle\mathrm{HF}\left|\left[X_{j_{1}}^{T_{0}},\left|\mu_{k}^{\mathrm{CC}}\right\rangle\langle\mathrm{HF}|\right]\right| \mathrm{HF}\right\rangle \\
& -\sum_{v_{m}} \bar{t}_{v_{m}}^{(0)}\left\langle v_{m}\left|\left[X_{j_{1}}^{T_{0}},\left|\mu_{k}^{\mathrm{CC}}\right\rangle\langle\mathrm{HF}|\right]\right| \mathrm{HF}\right\rangle,
\end{aligned}
$$


where for the left-hand side, we have used the condition $\omega_{X_{j_{1}}}+\omega_{X_{j_{2}}}=0$.

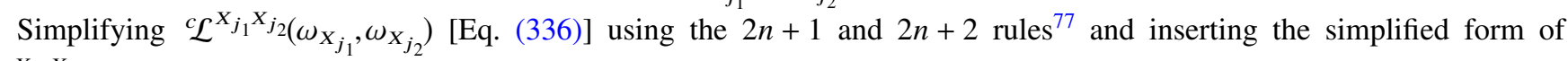

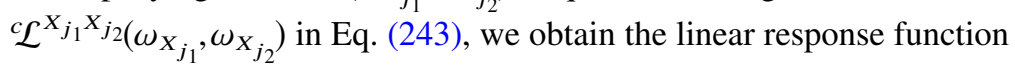

$$
\begin{aligned}
\left\langle\left\langle X_{j_{1}} ; X_{j_{2}}\right\rangle\right\rangle_{\omega_{X_{j_{2}}}}= & \frac{1}{2} C^{ \pm \omega} P_{\omega_{X_{j_{1}}} \omega_{X_{j_{2}}}}^{X_{j_{j_{2}} X_{j_{2}}}}\left(-\sum_{\mu_{k}} \bar{t}_{\mu_{k}}^{(0)} s_{\mu_{k}}^{X_{j_{1}}}\left(\omega_{X_{j_{1}}}\right) \sum_{v_{m}} s_{v_{\gamma_{m}}}^{X_{j_{2}}}\left(\omega_{X_{j_{2}}}\right)\left\langle\mathrm{HF}\left|H_{0}^{T_{0}}\right| v_{m}^{\mathrm{CC}}\right\rangle\right. \\
& \left.+\sum_{v_{m}} s_{v_{m}}^{X_{j_{2}}}\left(\omega_{X_{j_{2}}}\right)\left\langle\mathrm{HF}\left|\left[X_{j_{1}}^{T_{0}},\left|v_{m}^{\mathrm{CC}}\right\rangle\langle\mathrm{HF}|\right]\right| \mathrm{HF}\right\rangle+\sum_{\mu_{k} v_{m}} \bar{t}_{\mu_{k}}^{(0)} s_{v_{m}}^{X_{j_{2}}}\left(\omega_{X_{j_{2}}}\right)\left\langle\mu_{k}^{\mathrm{CC}}\left|\left[X_{j_{1}}^{T_{0}},\left|v_{m}^{\mathrm{CC}}\right\rangle\langle\mathrm{HF}|\right]\right| \mathrm{HF}\right\rangle\right), \quad \omega_{X_{j_{1}}}+\omega_{X_{j_{2}}}=0,
\end{aligned}
$$

where $t_{\mu_{k}}^{(0)}, \bar{t}_{\mu_{k}}^{(0)}$, and $s_{\mu_{k}}^{X_{j_{2}}}\left(\omega_{X_{j_{2}}}\right)$ are obtained by solving Eqs. (325), (327), and (338), respectively.

\section{Third-order molecular properties}

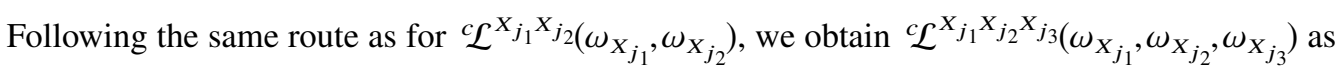

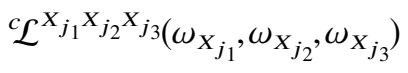

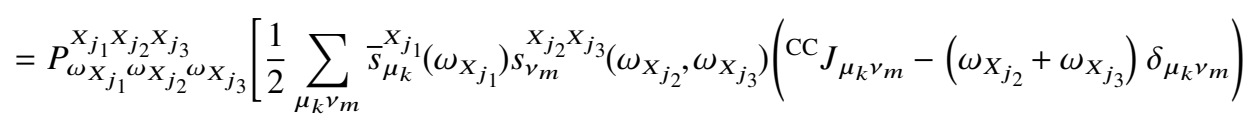

$$
\begin{aligned}
& +\frac{1}{2} \sum_{\mu_{k} v_{m}} \bar{s}_{\mu_{k}}^{X_{j_{1}} X_{j_{2}}}\left(\omega_{X_{j_{1}}}, \omega_{X_{j_{2}}}\right) s_{v_{m}}^{X_{j_{3}}}\left(\omega_{X_{j_{3}}}\right)\left({ }^{\mathrm{CC}} J_{\mu_{k} v_{m}}-\omega_{X_{j_{3}}} \delta_{\mu_{k} v_{m}}\right) \\
& -\frac{1}{2} \sum_{\mu_{k}} \bar{t}_{\mu_{k}}^{(0)} s_{\mu_{k}}^{X_{j_{1}}}\left(\omega_{X_{j_{1}}}\right) \sum_{v_{m}} s_{v_{\gamma_{m}}}^{X_{j_{2}} X_{j_{3}}}\left(\omega_{X_{j_{2}}}, \omega_{X_{j_{3}}}\right)\left\langle\mathrm{HF}\left|H_{0}^{T_{0}}\right| v_{m}^{\mathrm{CC}}\right\rangle \\
& -\frac{1}{2} \sum_{\mu_{k}} \bar{t}_{\mu_{k}}^{(0)} s_{\mu_{k}}^{X_{j_{1}} X_{j_{2}}}\left(\omega_{X_{j_{1}}}, \omega_{X_{j_{2}}}\right) \sum_{v_{m}} s_{v_{m}}^{X_{j_{3}}}\left(\omega_{X_{j_{3}}}\right)\left\langle\mathrm{HF}\left|H_{0}^{T_{0}}\right| v_{m}^{\mathrm{CC}}\right\rangle \\
& -\sum_{\mu_{k}} \bar{s}_{\mu_{k}}^{X_{j_{1}}}\left(\omega_{X_{j_{1}}}\right) s_{\mu_{k}}^{X_{j_{2}}}\left(\omega_{X_{j_{2}}}\right) \sum_{v_{m}}{ }^{X_{v_{m}}}{ }_{j_{j_{3}}}\left(\omega_{X_{j_{3}}}\right)\left\langle\mathrm{HF}\left|H_{0}^{T_{0}}\right| v_{m}^{\mathrm{CC}}\right\rangle \\
& +\frac{1}{2} \sum_{v_{m}} s_{v_{m}}^{X_{j_{2}} X_{j_{3}}}\left(\omega_{X_{j_{2}}}, \omega_{X_{j_{3}}}\right)\left\langle\mathrm{HF}\left|\left[X_{j_{1}}^{T_{0}},\left|v_{m}^{\mathrm{CC}}\right\rangle\langle\mathrm{HF}|\right]\right| \mathrm{HF}\right\rangle \\
& +\frac{1}{2} \sum_{\mu_{k}} \bar{s}_{\mu_{k}}^{X_{j_{2}} X_{j_{3}}}\left(\omega_{X_{j_{2}}}, \omega_{X_{j_{3}}}\right)\left\langle\mu_{k}^{\mathrm{CC}}\left|X_{j_{1}}^{T_{0}}\right| \mathrm{HF}\right\rangle \\
& +\frac{1}{2} \sum_{\mu_{k} v_{m}} \bar{t}_{\mu_{k}}^{(0)} s_{v_{m}}^{X_{j_{2}} X_{j_{3}}}\left(\omega_{X_{j_{2}}}, \omega_{X_{j_{3}}}\right)\left\langle\mu_{k}^{\mathrm{CC}}\left|\left[X_{j_{1}}^{T_{0}},\left|v_{m}^{\mathrm{CC}}\right\rangle\langle\mathrm{HF}|\right]\right| \mathrm{HF}\right\rangle \\
& +\sum_{\mu_{k} v_{m}} \bar{s}_{\mu_{k}}^{X_{j_{2}}}\left(\omega_{X_{j_{2}}}\right) s_{v_{m}}^{X_{j_{3}}}\left(\omega_{X_{j_{3}}}\right)\left\langle\mu_{k}^{\mathrm{CC}}\left|\left[X_{j_{1}}^{T_{0}},\left|v_{m}^{\mathrm{CC}}\right\rangle\langle\mathrm{HF}|\right]\right| \mathrm{HF}\right\rangle \\
& \left.-\sum_{\mu_{k}} \bar{t}_{\mu_{k}}^{(0)} s_{\mu_{k}}^{X_{j_{2}}}\left(\omega_{X_{j_{2}}}\right) \sum_{v_{m}} s_{v_{m}}^{X_{j_{3}}}\left(\omega_{X_{j_{3}}}\right)\left\langle\mathrm{HF}\left|X_{j_{1}}^{T_{0}}\right| v_{m}^{\mathrm{CC}}\right\rangle\right], \quad \omega_{X_{j_{1}}}+\omega_{X_{j_{2}}}+\omega_{X_{j_{3}}}=0 .
\end{aligned}
$$

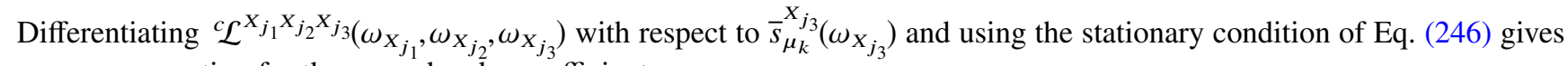
the response equation for the second-order coefficients, 


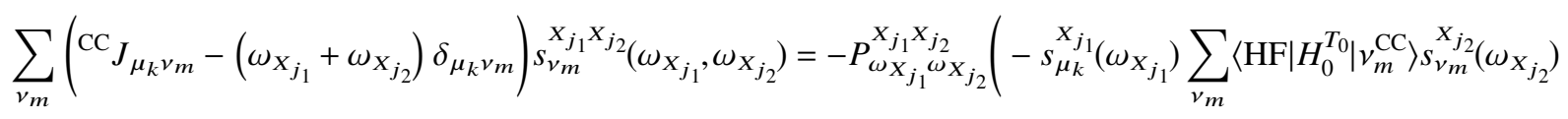

$$
\begin{aligned}
& \left.+\sum_{v_{m}}\left\langle\mu_{k}^{\mathrm{CC}}\left|\left[X_{j_{1}}^{T_{0}},\left|v_{m}^{\mathrm{CC}}\right\rangle\langle\mathrm{HF}|\right]\right| \mathrm{HF}\right\rangle_{v_{m}}^{X_{j_{2}}}\left(\omega_{X_{j_{2}}}\right)\right)
\end{aligned}
$$

from which $s_{v_{m}}^{X_{j_{1}} X_{j_{2}}}\left(\omega_{X_{j_{1}}}, \omega_{X_{j_{2}}}\right)$, needed in Subsection VII E for the fourth-order properties, may be calculated.

Using the $2 n+1$ and $2 n+2$ rules, the third-order molecular property expression becomes

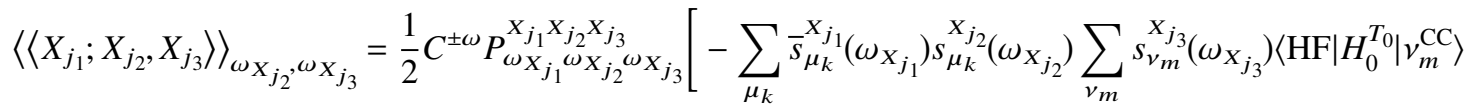

$$
\begin{aligned}
& +\sum_{\mu_{k} v_{m}} \bar{s}_{\mu_{k}}^{X_{j_{2}}}\left(\omega_{X_{j_{2}}}\right) s_{v_{m}}^{X_{j_{3}}}\left(\omega_{X_{j_{3}}}\right)\left\langle\mu_{k}^{\mathrm{CC}}\left|\left[X_{j_{1}}^{T_{0}},\left|v_{m}^{\mathrm{CC}}\right\rangle\langle\mathrm{HF}|\right]\right| \mathrm{HF}\right\rangle \\
& \left.-\sum_{\mu_{k}} \bar{t}_{\mu_{k}}^{(0)} s_{\mu_{k}}^{X_{j_{2}}}\left(\omega_{X_{j_{2}}}\right) \sum_{v_{m}} s_{v_{m}}^{X_{j_{3}}}\left(\omega_{X_{j_{3}}}\right)\left\langle\mathrm{HF}\left|X_{j_{1}}^{T_{0}}\right| v_{m}^{\mathrm{CC}}\right\rangle\right], \quad \omega_{X_{j_{1}}}+\omega_{X_{j_{2}}}+\omega_{X_{j_{3}}}=0
\end{aligned}
$$

where $t_{\mu_{k}}^{(0)}, \bar{t}_{\mu_{k}}^{(0)},{ }_{\mu_{\mu_{k}}}^{X_{j_{2}}}\left(\omega_{X_{j_{2}}}\right)$, and $\bar{s}_{\mu_{k}}^{X_{j_{2}}}\left(\omega_{X_{j_{2}}}\right)$ are obtained by solving Eqs. (325), (327), (338), and (339), respectively.

\section{E. Fourth-order molecular properties}

Using the $2 n+1$ and $2 n+2$ rules, fourth-order molecular properties may be expressed in terms of the first- and second-order coefficients and first-order multipliers as

$$
\begin{aligned}
& \left\langle\left\langle X_{j_{1}} ; X_{j_{2}}, X_{j_{3}}, X_{j_{4}}\right\rangle\right\rangle_{\omega_{X_{j_{2}}, \omega_{X_{j_{3}}}, \omega_{X_{j_{4}}}}}
\end{aligned}
$$

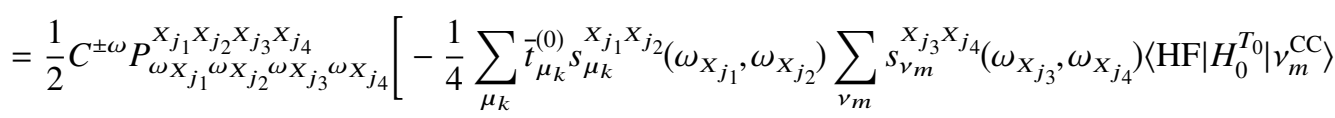

$$
\begin{aligned}
& -\frac{1}{2} \sum_{\mu_{k}} \bar{s}_{\mu_{k}}^{X_{j_{1}}}\left(\omega_{X_{j_{1}}}\right) s_{\mu_{k}}^{X_{j_{2}}}\left(\omega_{X_{j_{2}}}\right) \sum_{v_{m}} s_{v_{m}}^{X_{j_{3}} X_{j_{4}}}\left(\omega_{X_{j_{3}}}, \omega_{X_{j_{4}}}\right)\left\langle\mathrm{HF}\left|H_{0}^{T_{0}}\right| v_{m}^{\mathrm{CC}}\right\rangle \\
& -\frac{1}{2} \sum_{\mu_{k}} \bar{s}_{\mu_{k}}^{X_{j_{1}}}\left(\omega_{X_{j_{1}}}\right) s_{\mu_{k}}^{X_{j_{2}} X_{j_{3}}}\left(\omega_{X_{j_{2}}}, \omega_{X_{j_{3}}}\right) \sum_{v_{m}} s_{v_{m}}^{X_{j_{4}}}\left(\omega_{X_{j_{4}}}\right)\left\langle\mathrm{HF}\left|H_{0}^{T_{0}}\right| v_{m}^{\mathrm{CC}}\right\rangle \\
& +\frac{1}{2} \sum_{\mu_{k} v_{m}} \bar{s}_{\mu_{k}}^{X_{j_{2}}}\left(\omega_{X_{j_{2}}}\right) s_{v_{m}}^{X_{j_{3}} X_{j_{4}}}\left(\omega_{X_{j_{3}}}, \omega_{X_{j_{4}}}\right)\left\langle\mu_{k}^{\mathrm{CC}}\left|\left[X_{j_{1}}^{T_{0}},\left|v_{m}^{\mathrm{CC}}\right\rangle\langle\mathrm{HF}|\right]\right| \mathrm{HF}\right\rangle \\
& -\frac{1}{2} \sum_{\mu_{k}} \bar{t}_{\mu_{k}}^{(0)} s_{\mu_{k}}^{X_{j_{2}}}\left(\omega_{X_{j_{2}}}\right) \sum_{v_{m}} s_{v_{m}}^{X_{j_{3}} X_{j_{4}}}\left(\omega_{X_{j_{3}}}, \omega_{X_{j_{4}}}\right)\left\langle\mathrm{HF}\left|X_{j_{1}}^{T_{0}}\right| v_{m}^{\mathrm{CC}}\right\rangle \\
& -\frac{1}{2} \sum_{\mu_{k}} \bar{t}_{\mu_{k}}^{(0)} s_{\mu_{k}}^{X_{j_{2}} X_{j_{3}}}\left(\omega_{X_{j_{2}}}, \omega_{X_{j_{3}}}\right) \sum_{v_{m}} s_{v_{m}}^{X_{j_{4}}}\left(\omega_{X_{j_{4}}}\right)\left\langle\mathrm{HF}\left|X_{j_{1}}^{T_{0}}\right| v_{m}^{\mathrm{CC}}\right\rangle \\
& \left.-\sum_{\mu_{k}} \bar{s}_{\mu_{k}}^{X_{j_{2}}}\left(\omega_{X_{j_{2}}}\right) s_{\mu_{k}}^{X_{j_{3}}}\left(\omega_{X_{j_{3}}}\right) \sum_{v_{m}} s_{v_{m}}^{X_{j_{4}}}\left(\omega_{X_{j_{4}}}\right)\left\langle\mathrm{HF}\left|X_{j_{1}}^{T_{0}}\right| v_{m}^{\mathrm{CC}}\right\rangle\right], \quad \omega_{X_{j_{1}}}+\omega_{X_{j_{2}}}+\omega_{X_{j_{3}}}+\omega_{X_{j_{4}}}=0,
\end{aligned}
$$

where $t_{\mu_{k}}^{(0)}, \bar{t}_{\mu_{k}}^{(0)}, s_{\mu_{k}}^{X_{j_{1}}}\left(\omega_{X_{j_{1}}}\right), \bar{s}_{\mu_{k}}^{X_{j_{1}}}\left(\omega_{X_{j_{1}}}\right)$, and $s_{\mu_{k}}^{X_{j_{2}} X_{j_{3}}}\left(\omega_{X_{j_{2}}}, \omega_{X_{j_{2}}}\right)$ are obtained by solving Eqs. (325), (327), (338), (339), and (342), respectively.

\section{F. The $\mathrm{CC}$ Jacobian as a part of the $\mathrm{CC}-\mathrm{Cl}$ eigenvalue equation}

We now consider a matrix representation of the eigenvalue equation for the unperturbed Hamiltonian in the basis of
Eq. (332) in which the CC-CI time-dependent wave function is expanded [Eq. (331)]. We then express the Jacobian matrix and its eigenvalue equation in this basis.

\section{The $\mathrm{CC}$ - $\mathrm{Cl}$ eigenvalue equation}

The time development of the CC-CI wave function in Eq. (331) is described in terms of a linear expansion in the basis $\left|\mathcal{B}^{T_{0}}\right\rangle$ of Eq. (332). We now determine a matrix representation 
of the unperturbed Hamiltonian $H_{0}$ considering $\left|\mathcal{B}^{T_{0}}\right\rangle$ as the right basis and using its biorthonormal counterpart

$$
\left\langle\mathcal{B}^{T_{0}}\right|=\left\{\langle\mathrm{HF}| \exp \left(-T_{0}\right),\left\langle\mu_{k}^{\mathrm{CC}}\right| \exp \left(-T_{0}\right)\right\}
$$

as the left basis. In this biorthonormal representation, the eigenvalue equation for the unperturbed Hamiltonian becomes

$$
\begin{aligned}
{ }^{\mathrm{CC}} \mathbf{H}_{0}^{T_{0}{ }^{\mathrm{CC}}} \mathbf{C}^{\mathrm{R}}={ }^{\mathrm{CC}} \mathbf{C}^{\mathrm{R} C \mathrm{CC}} \mathbf{E}, \\
{ }^{\mathrm{CC}} \mathbf{C}^{\mathrm{L}{ }^{\mathrm{CC}}} \mathbf{H}_{0}^{T_{0}}={ }^{\mathrm{CC}} \mathbf{E}{ }^{\mathrm{CC}} \mathbf{C}^{\mathrm{L}},
\end{aligned}
$$

where

$$
{ }^{\mathrm{CC}} \mathbf{C}^{\mathrm{L} C C} \mathbf{C}^{\mathrm{R}}=\mathbf{I} .
$$

The unperturbed Hamiltonian matrix, ${ }^{\mathrm{C}} \mathbf{H}_{0}^{T_{0}}$, has the block structure

$$
{ }^{\mathrm{CC}} \mathbf{H}_{0}^{T_{0}}=\left(\begin{array}{cc}
{ }^{\mathrm{CC}} E_{0} & \boldsymbol{\eta} \\
\mathbf{0} & \left({ }^{\mathrm{CC}} \mathbf{H}_{0}^{T_{0}}{ }^{\perp}\right.
\end{array}\right),
$$

where $\left({ }^{\mathrm{CC}} \mathbf{H}_{0}^{T_{0}}\right)^{\perp}$ denotes the sub-block of the Hamiltonian matrix in the orthogonal complement subspace

$$
\begin{aligned}
\left({ }^{\mathrm{CC}} \mathbf{H}_{0}^{T_{0}}\right)_{\mu_{k} v_{m}}^{\perp} & =\left[{ }^{\mathrm{CC}} \mathbf{H}_{0}^{T_{0}}\right]_{\mu_{k} v_{m}} \\
& =\left\langle\mu_{k}^{\mathrm{CC}}\left|\exp \left(-T_{0}\right) H_{0} \exp \left(T_{0}\right)\right| v_{m}^{\mathrm{CC}}\right\rangle \\
& =\left\langle\mu_{k}^{\mathrm{CC}}\left|H_{0}^{T_{0}}\right| v_{m}^{\mathrm{CC}}\right\rangle,
\end{aligned}
$$

where we have used Eq. (191). To obtain the block structure in Eq. (349), we have used Eqs. (324), (325), and (329),

$$
\begin{aligned}
& {\left[{ }^{\mathrm{CC}} \mathbf{H}_{0}^{T_{0}}\right]_{\mathrm{HF} \mathrm{HF}}=\left\langle\mathrm{HF}\left|\exp \left(-T_{0}\right) H_{0} \exp \left(T_{0}\right)\right| \mathrm{HF}\right\rangle} \\
& =\left\langle\mathrm{HF}\left|H_{0}^{T_{0}}\right| \mathrm{HF}\right\rangle={ }^{\mathrm{CC}} E_{0}, \\
& {\left[{ }^{\mathrm{CC}} \mathbf{H}_{0}^{T_{0}}\right]_{\mu_{k} \mathrm{HF}}=\left\langle\mu_{k}^{\mathrm{CC}}\left|\exp \left(-T_{0}\right) H_{0} \exp \left(T_{0}\right)\right| \mathrm{HF}\right\rangle} \\
& =\left\langle\mu_{k}^{\mathrm{CC}}\left|H_{0}^{T_{0}}\right| \mathrm{HF}\right\rangle=0, \\
& \left.{ }^{\mathrm{CC}} \mathbf{H}_{0}^{T_{0}}\right]_{\mathrm{HF} \mu_{k}}=\left\langle\mathrm{HF}\left|\exp \left(-T_{0}\right) H_{0} \exp \left(T_{0}\right)\right| \mu_{k}^{\mathrm{CC}}\right\rangle \\
& =\left\langle\mathrm{HF}\left|H_{0}^{T_{0}}\right| \mu_{k}^{\mathrm{CC}}\right\rangle=\eta_{\mu_{k}} .
\end{aligned}
$$

The ${ }^{\mathrm{CC}} \mathbf{E}$ matrix is diagonal and contains the energies of the unperturbed Hamiltonian $H_{0}$,

$$
{ }^{\mathrm{CC}} \mathbf{E}=\left(\begin{array}{cccc}
{ }^{\mathrm{CC}} E_{0} & & & \\
& { }^{\mathrm{CC}} E_{1} & & \\
& & { }^{C C} E_{2} & \\
& & & \ddots
\end{array}\right) .
$$

Equations (350)-(353) imply that Eq. (349) may be viewed as a matrix representation of the similarity-transformed Hamiltonian, $H_{0}^{T_{0}}$ [Eq. (191)], in the basis $|\mathcal{B}\rangle$ [Eq. (323)]. As the similarity-transformed Hamiltonian, $H_{0}^{T_{0}}$, is nonHermitian, so is its matrix representation and therefore the left and right eigenvectors, contained in ${ }^{\mathrm{CC}} \mathrm{C}^{\mathrm{L}}$ [Eq. (346)] and ${ }^{\mathrm{CC}} \mathrm{C}^{\mathrm{R}}$ [Eq. (347)] matrices, respectively, are not adjoints of each other. To obtain explicit expressions for the left and right eigenvectors, we use the block structure of ${ }^{\mathrm{CC}} \mathbf{H}_{0}^{T_{0}}$ [Eq. (349)] to write the ${ }^{\mathrm{CC}} \mathbf{C}^{\mathrm{R}}$ and ${ }^{\mathrm{CC}} \mathbf{C}^{\mathrm{L}}$ matrices as

$$
{ }^{\mathrm{CC}} \mathbf{C}^{\mathrm{R}}=\left(\begin{array}{ll}
{ }^{\mathrm{CC}} D_{0} & { }^{\mathrm{CC}} \mathbf{D} \\
{ }^{\mathrm{CC}} \mathbf{C}_{0} & { }^{\mathrm{CC}} \mathbf{C}
\end{array}\right)
$$

and

$$
{ }^{\mathrm{CC}} \mathbf{C}^{\mathrm{L}}=\left(\begin{array}{cc}
{ }^{\mathrm{C}} \bar{D}_{0} & { }^{\mathrm{CC}} \overline{\mathbf{C}}_{0} \\
{ }^{\mathrm{C}} \overline{\mathbf{D}} & { }^{\mathrm{CC}} \overline{\mathbf{C}}
\end{array}\right) .
$$

Inserting Eqs. (355) and (356) in Eqs. (346)-(348), we obtain, after some algebra (see, for example, Ref. 88), the ground state eigenvectors as

$$
\begin{gathered}
{ }^{{ }^{C}} \mathbf{C}_{0}^{\mathrm{R}}=\left(\begin{array}{l}
1 \\
\mathbf{0}
\end{array}\right), \\
{ }^{\mathrm{C}}{ }_{\mathbf{C}_{0}}^{\mathrm{L}}=\left(\begin{array}{ll}
1 & \overline{\mathbf{t}}^{(0)}
\end{array}\right),
\end{gathered}
$$

whereas the excited state eigenvectors for the energies ${ }^{\mathrm{CI}} E_{n}$, $n=1,2, \ldots$, become

$$
\begin{aligned}
{ }^{\mathrm{CC}} \mathbf{C}_{n}^{\mathrm{R}} & =\left(\begin{array}{cc}
-\overline{\mathbf{t}}^{(0)}{ }^{\mathrm{CC}} \mathbf{C}_{n} \\
{ }^{\mathrm{CC}} \mathbf{C}_{n}
\end{array}\right), \\
{ }^{\mathrm{CC}} \mathbf{C}_{n}^{\mathrm{L}} & =\left(\begin{array}{ll}
0 & { }^{\mathrm{CC}} \overline{\mathbf{C}}_{n}
\end{array}\right),
\end{aligned}
$$

where $\overline{\mathbf{t}}^{(0)}$ is obtained from Eq. (327). Further, ${ }^{\mathrm{CC}} \mathbf{C}_{n}$ and ${ }^{\mathrm{CC}} \overline{\mathbf{C}}_{n}$ are the right and left eigenvectors of $\left({ }^{\mathrm{C} C} \mathbf{H}_{0}^{T_{0}}\right)^{\perp}$,

$$
\begin{aligned}
& \left({ }^{\mathrm{CC}} \mathbf{H}_{0}^{T_{0}}\right)^{\perp}{ }^{\mathrm{CC}} \mathbf{C}={ }^{\mathrm{CC}} \mathbf{C}{ }^{\mathrm{CC}} \mathbf{E}^{\perp}, \\
& { }^{\mathrm{CC}} \overline{\mathbf{C}}\left({ }^{\mathrm{CC}} \mathbf{H}_{0}^{T_{0}}\right)^{\perp}={ }^{{ }^{C C}} \mathbf{E}^{\perp}{ }^{\mathrm{CC}} \overline{\mathbf{C}},
\end{aligned}
$$

where

$$
{ }^{\mathrm{C}} \overline{\mathbf{C}}{ }^{\mathrm{CC}} \mathbf{C}=\mathbf{I}
$$

and ${ }^{\mathrm{CC}} \mathbf{E}^{\perp}$ is the sub-block of ${ }^{\mathrm{CC}} \mathbf{E}$ [Eq. (354)] in the orthogonal complement subspace.

Using Eqs. (357)-(360), we may express the right and left eigenstates of the standard Hamiltonian $H_{0}$ (without similarity transformation) in the bracket form as

$$
\begin{aligned}
& \left|0_{\mathrm{R}}^{\mathrm{CC}}\right\rangle=\left\{\left|0_{0}^{\mathrm{CC}}\right\rangle,\left|0_{1}^{\mathrm{CC}}\right\rangle,\left|0_{2}^{\mathrm{CC}}\right\rangle, \cdots\right\}, \\
& \left\langle 0_{\mathrm{L}}^{\mathrm{CC}}\right|=\left\{\left\langle 0_{0}^{\mathrm{CC}}\right|,\left\langle 0_{1}^{\mathrm{CC}}\right|,\left\langle 0_{2}^{\mathrm{CC}}\right|, \cdots\right\},
\end{aligned}
$$

where

$$
\begin{gathered}
\left|0_{0}^{\mathrm{CC}}\right\rangle=\exp \left(T_{0}\right)|\mathrm{HF}\rangle, \\
\left\langle 0_{0}^{\mathrm{CC}}\right|=\langle\mathrm{HF}| \exp \left(-T_{0}\right)+\sum_{\mu_{k}} \bar{t}_{\mu_{k}}^{(0)}\left\langle\mu_{k}^{\mathrm{CC}}\right| \exp \left(-T_{0}\right),
\end{gathered}
$$

and, for $n \neq 0$,

$$
\begin{aligned}
\left|0_{n}^{\mathrm{CC}}\right\rangle= & -\sum_{\mu_{k}} \bar{t}_{\mu_{k}}^{(0)}{ }^{\mathrm{CC}} C_{\mu_{k} n} \exp \left(T_{0}\right)|\mathrm{HF}\rangle \\
& +\sum_{\mu_{k}}{ }^{\mathrm{CC}} C_{\mu_{k} n} \exp \left(T_{0}\right)\left|\mu_{k}^{\mathrm{CC}}\right\rangle
\end{aligned}
$$




$$
\left\langle 0_{n}^{\mathrm{CC}}\right|=\sum_{\mu_{k}}{ }^{\mathrm{CC}} \bar{C}_{n \mu_{k}}\left\langle\mu_{k}^{\mathrm{CC}}\right| \exp \left(-T_{0}\right),
$$

and where ${ }^{\mathrm{CC}} C_{\mu_{k} n}$ are elements of the ${ }^{\mathrm{CC}} \mathbf{C}_{n}$ vector [Eq. (359)], whereas ${ }^{\mathrm{CC}} \bar{C}_{n \mu_{k}}$ are elements of ${ }^{\mathrm{CC}} \overline{\mathbf{C}}_{n}$ [Eq. (360)].

The eigenvalue equations defined by Eqs. (346)-(354) are often referred to as the EOMCC eigenvalue equation ${ }^{88}$ and form the foundation for the EOMCC model, which will be discussed in Sec. IX.

\section{The CC Jacobian eigenvalue equation}

We now consider the CC-CI Jacobian or, equivalently, the CC Jacobian [see Eq. (337)]. Using Eqs. (328) and (350), we may express the CC Jacobian matrix as

$$
{ }^{\mathrm{CC}} \mathbf{J}=\left({ }^{\mathrm{CC}} \mathbf{H}_{0}^{T_{0}}\right)^{\perp}-{ }^{\mathrm{CC}} E_{0} \mathbf{I} .
$$

Using Eq. (370), we may write Eqs. (361) and (362) as

$$
\begin{aligned}
& { }^{\mathrm{CC}} \mathbf{J}{ }^{\mathrm{CC}} \mathbf{C}={ }^{\mathrm{CC}} \mathbf{C} \boldsymbol{\Delta}^{\mathrm{CC}} \mathbf{E}, \\
& { }^{\mathrm{CC}} \overline{\mathbf{C}}{ }^{\mathrm{CC}} \mathbf{J}=\boldsymbol{\Delta}^{\mathrm{CC}} \mathbf{E}{ }^{\mathrm{CC}} \overline{\mathbf{C}},
\end{aligned}
$$

where ${ }^{\mathrm{CC}} \overline{\mathbf{C}}$ and ${ }^{\mathrm{CC}} \mathbf{C}$ are orthonormal [Eq. (363)] and where

$$
\begin{aligned}
\boldsymbol{\Delta}^{\mathrm{CC}} \mathbf{E} & ={ }^{\mathrm{CC}} \mathbf{E}^{\perp}-{ }^{\mathrm{CC}} E_{0} \mathbf{I} \\
& =\left(\begin{array}{lllll}
{ }^{\mathrm{CC}} E_{10} 0 & & & \\
& { }^{C C} E_{20} & & \\
& & { }^{C C} E_{30} & \\
& & & \ddots
\end{array}\right),
\end{aligned}
$$

with

$$
{ }^{\mathrm{CC}} E_{n 0}={ }^{\mathrm{CC}} E_{n}-{ }^{\mathrm{CC}} E_{0} .
$$

Equations (371)-(374) show that the eigenvalues of ${ }^{\mathrm{CC}} \mathbf{J}$ are equal to the excitation energies of the unperturbed system.

Comparing Eqs. (371) and (372) with Eqs. (361) and (362), we see that the eigenvectors of the CC Jacobian, ${ }^{\mathrm{CC}} \mathbf{J}$, are equal to the eigenvectors of the CC Hamiltonian matrix, $\left({ }^{\mathrm{CC}} \mathbf{H}_{0}^{T_{0}}\right)^{\perp}$, in Eq. (349). The excited states are thus linearly parametrized in the biorthonormal basis $\left|\mathcal{B}^{T_{0}}\right\rangle$ [Eq. (332)] and $\left\langle\mathcal{B}^{T_{0}}\right|$ [Eq. (345)] as is the time evolution of the CC ground state [Eq. (331)]. As there is no interaction between the ground state and the orthogonal complement set of states, neither for the unperturbed system nor for the time-evolving system, we therefore have an explicit representation of the excited states in CC-CI response theory.

\section{MOLECULAR PROPERTIES FOR THE CC MODEL}

\section{A. Parametrization of the CC model}

In the $\mathrm{CC}$ model, the unperturbed ground state, $\exp \left(T_{0}\right)|\mathrm{HF}\rangle$, satisfies Eqs. (323)-(327), and the time evolution is expo- nentially parametrized in terms of the time-dependent cluster operator

$$
T(t)=\sum_{\mu_{k}} t_{\mu_{k}}(t) \tau_{\mu_{k}},
$$

where $t_{\mu_{k}}(t)$ are time-dependent cluster amplitudes. The CC wave function $\left|0_{I}^{\mathrm{CC}}\right\rangle$ [Eq. (253)] becomes

$$
\begin{aligned}
\left|0_{I}^{\mathrm{CC}}\right\rangle= & \exp \left(T_{0}\right) \exp (T(t))|\mathrm{HF}\rangle \\
= & \exp \left(T_{0}\right)|\mathrm{HF}\rangle+\exp \left(T_{0}\right) T(t)|\mathrm{HF}\rangle \\
& +\frac{1}{2} \exp \left(T_{0}\right) T^{2}(t)|\mathrm{HF}\rangle+\cdots \\
= & \exp \left(T_{0}\right)|\mathrm{HF}\rangle+\sum_{\mu_{k}} t_{\mu_{k}}(t) \exp \left(T_{0}\right)\left|\mu_{k}^{\mathrm{CC}}\right\rangle \\
& +\frac{1}{2} \sum_{\mu_{k} v_{m}} t_{\mu_{k}}(t) t_{v_{m}}(t) \exp \left(T_{0}\right)\left|\mu_{k} v_{m}^{\mathrm{CC}}\right\rangle+\cdots
\end{aligned}
$$

Equation (376) shows that for a complete basis $\left\{|\mathrm{HF}\rangle,\left|\mu_{k}^{\mathrm{CC}}\right\rangle\right\}$ [Eq. (323)], the wave function $\left|0_{I}^{\mathrm{CC}}\right\rangle$ describes a non-linear parametrization of the time evolution in this basis. If a truncation is carried out in the basis $\left\{|\mathrm{HF}\rangle,\left|\mu_{k}^{\mathrm{CC}}\right\rangle\right\}$, then the time evolution in the CC model is expanded in a basis that is larger than the one of the CC-CI model [Eqs. (331) and (332)] which contains only a linearized form of Eq. (376).

We now follow the same route as in Sec. VII to derive explicit expressions for the $\mathrm{CC}$ molecular response functions through fourth order and use the relabeling $|\mathscr{R}\rangle=|\mathrm{HF}\rangle,|k\rangle$ $=\left|\mu_{k}^{\mathrm{CC}}\right\rangle, B_{0}=T_{0}, b_{k}=t_{\mu_{k}}$, and $\bar{b}_{k}=\bar{t}_{\mu_{k}}$ in ${ }^{c} \mathcal{L}_{\mathcal{H}}$ [Eq. (273)] and ${ }^{c} \mathcal{L}_{X_{j_{1}}}$ [Eq. (272)].

\section{B. Energy and first-order molecular properties}

From Eq. (273), we see that ${ }^{c} \mathcal{L}^{(0)}$ is equal to the standard ground-state $\mathrm{CC}$ energy,

$$
{ }^{c} \mathcal{L}^{(0)}={ }^{c} \mathcal{L}_{\mathcal{H}}^{(0)}={ }^{c} \mathcal{L}_{H_{0}}^{(0)}={ }^{\mathrm{CC}} E_{0}=\left\langle\mathrm{HF}\left|H_{0}^{T_{0}}\right| \mathrm{HF}\right\rangle,
$$

since the amplitudes $t_{\mu_{k}}\left(\omega_{K}\right)$ do not contain zero-order contributions.

The first-order complex quasi-energy Lagrangian is obtained using Eqs. (272) and (273) as

$$
{ }^{c} \mathcal{L}^{X_{j_{1}}(0)}=\left\langle\mathrm{HF}\left|X_{j_{1}}^{T_{0}}\right| \mathrm{HF}\right\rangle+\sum_{\mu_{k}} \bar{t}_{\mu_{k}}^{(0)}\left\langle\mu_{k}^{\mathrm{CC}}\left|X_{j_{1}}^{T_{0}}\right| \mathrm{HF}\right\rangle,
$$

which, inserted in Eq. (243), gives the first-order molecular properties as

$$
\begin{aligned}
\left\langle\left\langle X_{j_{1}}\right\rangle\right\rangle_{0}= & \frac{1}{2} C^{ \pm \omega}\left(\left\langle\mathrm{HF}\left|X_{j_{1}}^{T_{0}}\right| \mathrm{HF}\right\rangle\right. \\
& \left.+\sum_{\mu_{k}} \bar{t}_{\mu_{k}}^{(0)}\left\langle\mu_{k}^{\mathrm{CC}}\left|X_{j_{1}}^{T_{0}}\right| \mathrm{HF}\right\rangle\right),
\end{aligned}
$$

where the zero-order amplitudes, $t_{\mu_{k}}^{(0)}$, and the zero-order multipliers, $\bar{t}_{\mu_{k}}^{(0)}$, are obtained by solving Eqs. (325) and (327), respectively. 


\section{Second-order molecular properties}

The second-order complex quasi-energy Lagrangian is obtained using Eqs. (272) and (273),

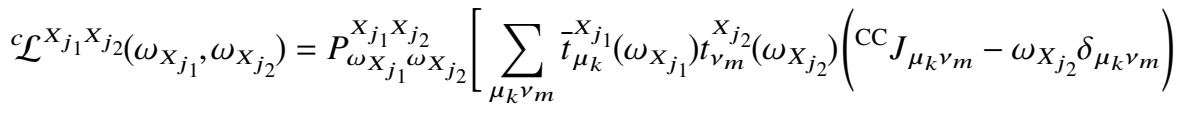

$$
\begin{aligned}
& +\frac{1}{2} \sum_{v_{m} \lambda_{n}} t_{v_{m}}^{X_{j_{1}}}\left(\omega_{X_{j_{1}}}\right) t_{\lambda_{n}}^{X_{j_{2}}}\left(\omega_{X_{j_{2}}}\right)\left\langle\mathrm{HF}\left|\left[\left[H_{0}^{T_{0}}, \tau_{v_{m}}\right], \tau_{\lambda_{n}}\right]\right| \mathrm{HF}\right\rangle \\
& +\frac{1}{2} \sum_{\mu_{k} v_{m} \lambda_{n}} \bar{t}_{\mu_{k}}^{(0)} t_{\nu_{m}}^{X_{j_{1}}}\left(\omega_{X_{j_{1}}}\right) t_{\lambda_{n}}^{X_{j_{2}}}\left(\omega_{X_{j_{2}}}\right)\left\langle\mu_{k}^{\mathrm{CC}}\left|\left[\left[H_{0}^{T_{0}}, \tau_{v_{m}}\right], \tau_{\lambda_{n}}\right]\right| \mathrm{HF}\right\rangle \\
& +\sum_{v_{m}} t_{v_{m}}^{X_{j_{2}}}\left(\omega_{X_{j_{2}}}\right)\left\langle\mathrm{HF}\left|\left[X_{j_{1}}^{T_{0}}, \tau_{v_{m}}\right]\right| \mathrm{HF}\right\rangle \\
& \left.+\sum_{\mu_{k}} \bar{t}_{\mu_{k}}^{X_{j_{2}}}\left(\omega_{X_{j_{2}}}\right)\left\langle\mu_{k}^{\mathrm{CC}}\left|X_{j_{1}}^{T_{0}}\right| \mathrm{HF}\right\rangle+\sum_{\mu_{k} v_{m}} \bar{t}_{\mu_{k}}^{(0)} t_{v_{m}}^{X_{j_{2}}}\left(\omega_{X_{j_{2}}}\right)\left\langle\mu_{k}^{\mathrm{CC}}\left[X_{j_{1}}^{T_{0}}, \tau_{v_{m}}\right] \mid \mathrm{HF}\right\rangle\right], \quad \omega_{X_{j_{1}}}+\omega_{X_{j_{2}}}=0,
\end{aligned}
$$

where the CC Jacobian, ${ }^{\mathrm{CC}} J_{\mu_{k} v_{m}}$, has been given in Eq. (328).

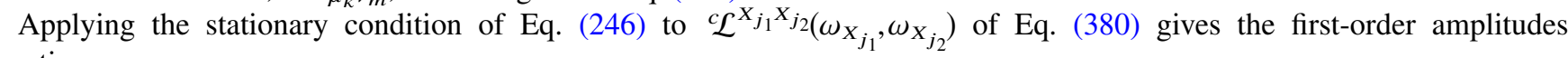
equation

$$
\sum_{v_{m}}\left({ }^{\mathrm{CC}} J_{\mu_{k} v_{m}}-\omega_{X_{j_{1}}} \delta_{\mu_{k} v_{m}}\right) t_{v_{m}}^{X_{j_{1}}}\left(\omega_{X_{j_{1}}}\right)=-\left\langle\mu_{k}^{\mathrm{CC}}\left|X_{j_{1}}^{T_{0}}\right| \mathrm{HF}\right\rangle
$$

Similarly, applying the stationary condition of Eq. (245) gives the first-order multipliers equation

$$
\begin{aligned}
& \sum_{v_{m}} \bar{t}_{v_{m}}^{X_{j_{1}}}\left(\omega_{X_{j_{1}}}\right)\left({ }^{\mathrm{CC}}{\left.J_{v_{m} \mu_{k}}+\omega_{X_{j_{1}}} \delta_{v_{m} \mu_{k}}\right)=}-\sum_{v_{m}} t_{v_{m}}^{X_{j_{1}}}\left(\omega_{X_{j_{1}}}\right)\left\langle\mathrm{HF}\left|\left[\left[H_{0}^{T_{0}}, \tau_{v_{m}}\right], \tau_{\mu_{k}}\right]\right| \mathrm{HF}\right\rangle\right. \\
&-\sum_{v_{m} \lambda_{n}} \bar{t}_{v_{m}}^{(0)} t_{\lambda_{n}}^{X_{j_{1}}}\left(\omega_{X_{j_{1}}}\right)\left\langle v_{m}^{\mathrm{CC}}\left|\left[\left[H_{0}^{T_{0}}, \tau_{\lambda_{n}}\right], \tau_{\mu_{k}}\right]\right| \mathrm{HF}\right\rangle-\left\langle\mathrm{HF}\left|\left[X_{j_{1}}^{T_{0}}, \tau_{\mu_{k}}\right]\right| \mathrm{HF}\right\rangle \\
&-\sum_{v_{m}} \bar{t}_{v_{m}}^{(0)}\left\langle v_{m}^{\mathrm{CC}}\left|\left[X_{j_{1}}^{T_{0}}, \tau_{\mu_{k}}\right]\right| \mathrm{HF}\right\rangle
\end{aligned}
$$

where for the left-hand side, we have used the condition $\omega_{X_{j_{1}}}+\omega_{X_{j_{2}}}=0$.

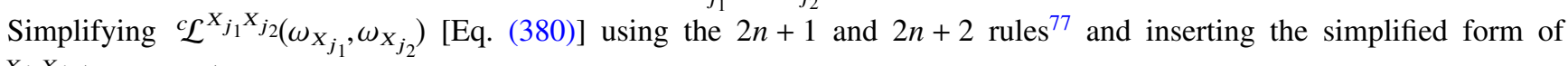

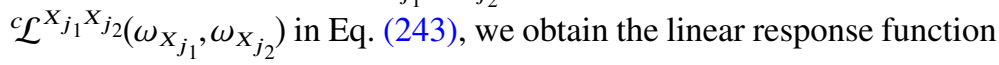

$$
\begin{aligned}
& \left\langle\left\langle X_{j_{1}} ; X_{j_{2}}\right\rangle\right\rangle_{\omega_{X_{j_{2}}}}=\frac{1}{2} C^{ \pm \omega} P_{\omega_{X_{j_{1}}} \omega_{X_{j_{2}}}}^{X_{j_{1}} X_{j_{2}}}\left[\frac{1}{2} \sum_{v_{m} \lambda_{n}} t_{v_{m}}^{X_{j_{1}}}\left(\omega_{X_{j_{1}}}\right) t_{\lambda_{n}}^{X_{j_{2}}}\left(\omega_{X_{j_{2}}}\right)\left\langle\mathrm{HF}\left|\left[\left[H_{0}^{T_{0}}, \tau_{v_{m}}\right], \tau_{\lambda_{n}}\right]\right| \mathrm{HF}\right\rangle\right. \\
& +\frac{1}{2} \sum_{\mu_{k} v_{m} \lambda_{n}} \bar{t}_{\mu_{k}}^{(0)} t_{v_{m}}^{X_{j_{1}}}\left(\omega_{X_{j_{1}}}\right) t_{\lambda_{n}}^{X_{j_{2}}}\left(\omega_{X_{j_{2}}}\right)\left\langle\mu_{k}^{\mathrm{CC}}\left|\left[\left[H_{0}^{T_{0}}, \tau_{v_{m}}\right], \tau_{\lambda_{n}}\right]\right| \mathrm{HF}\right\rangle \\
& \left.+\sum_{v_{m}} t_{v_{m}}^{X_{j_{2}}}\left(\omega_{X_{j_{2}}}\right)\left\langle\mathrm{HF}\left|\left[X_{j_{1}}^{T_{0}}, \tau_{v_{m}}\right]\right| \mathrm{HF}\right\rangle+\sum_{\mu_{k} v_{m}} \bar{t}_{\mu_{k}}^{(0)} t_{v_{m}}^{X_{j_{2}}}\left(\omega_{X_{j_{2}}}\right)\left\langle\mu_{k}^{\mathrm{CC}}\left|\left[X_{j_{1}}^{T_{0}}, \tau_{v_{m}}\right]\right| \mathrm{HF}\right\rangle\right], \quad \omega_{X_{j_{1}}}+\omega_{X_{j_{2}}}=0
\end{aligned}
$$

where $t_{\mu_{k}}^{(0)}, \bar{t}_{\mu_{k}}^{(0)}$, and $t_{\mu_{k}}^{X_{j_{2}}}\left(\omega_{X_{j_{2}}}\right.$ ) are obtained by solving Eqs. (325), (327), and (381), respectively. 


\section{Third-order molecular properties}

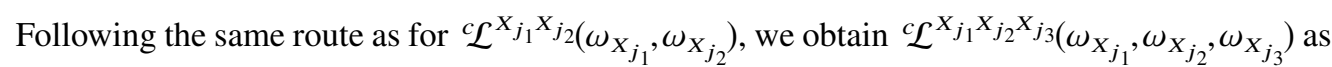

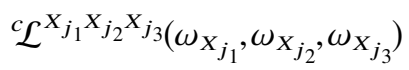

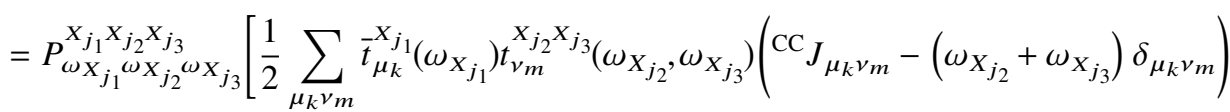

$$
\begin{aligned}
& +\frac{1}{2} \sum_{\mu_{k} v_{m}} \bar{t}_{\mu_{k}}^{X_{j_{1}} X_{j_{2}}}\left(\omega_{X_{j_{1}}}, \omega_{X_{j_{2}}}\right) t_{v_{m}}^{X_{j_{3}}}\left(\omega_{X_{j_{3}}}\right)\left({ }^{\mathrm{CC}} J_{\mu_{k} v_{m}}-\omega_{X_{j_{3}}} \delta_{\mu_{k} v_{m}}\right) \\
& +\frac{1}{2} \sum_{v_{m} \lambda_{n}} t_{v_{m}}^{X_{j_{1}}}\left(\omega_{X_{j_{1}}}\right) t_{\lambda_{n}}^{X_{j_{2}} X_{j_{3}}}\left(\omega_{X_{j_{2}}}, \omega_{X_{j_{3}}}\right)\left\langle\mathrm{HF}\left|\left[\left[H_{0}^{T_{0}}, \tau_{v_{m}}\right], \tau_{\lambda_{n}}\right]\right| \mathrm{HF}\right\rangle \\
& +\frac{1}{2} \sum_{\mu_{k} v_{m} \lambda_{n}} \bar{t}_{\mu_{k}}^{(0)} t_{v_{m}}^{X_{j_{1}}}\left(\omega_{X_{j_{1}}}\right) t_{\lambda_{n}}^{X_{j_{2}} X_{j_{3}}}\left(\omega_{X_{j_{2}}}, \omega_{X_{j_{3}}}\right)\left\langle\mu_{k}^{\mathrm{CC}}\left|\left[\left[H_{0}^{T_{0}}, \tau_{v_{m}}\right], \tau_{\lambda_{n}}\right]\right| \mathrm{HF}\right\rangle
\end{aligned}
$$

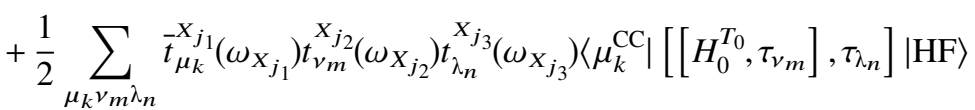

$$
\begin{aligned}
& +\frac{1}{6} \sum_{\mu_{k} v_{m} \lambda_{n} \sigma_{p}} \bar{t}_{\mu_{k}}^{(0)} t_{v_{m}}^{X_{j_{1}}}\left(\omega_{X_{j_{1}}}\right) t_{\lambda_{n}}^{X_{j_{2}}}\left(\omega_{X_{j_{2}}}\right) t_{\sigma_{p}}^{X_{j_{3}}}\left(\omega_{X_{j_{3}}}\right)\left\langle\mu_{k}^{\mathrm{CC}}\left|\left[\left[\left[H_{0}^{T_{0}}, \tau_{v_{m}}\right], \tau_{\lambda_{n}}\right], \tau_{\sigma_{p}}\right]\right| \mathrm{HF}\right\rangle \\
& +\frac{1}{2} \sum_{v_{m}} t_{v_{m}}^{X_{j_{2}} X_{j_{3}}}\left(\omega_{X_{j_{2}}}, \omega_{X_{j_{3}}}\right)\left\langle\mathrm{HF}\left|\left[X_{j_{1}}^{T_{0}}, \tau_{v_{m}}\right]\right| \mathrm{HF}\right\rangle+\frac{1}{2} \sum_{\mu_{k}}{ }_{t_{\mu_{k}}}^{X_{j_{2}} X_{j_{3}}}\left(\omega_{X_{j_{2}}}, \omega_{X_{j_{3}}}\right)\left\langle\mu_{k}^{\mathrm{CC}}\left|X_{j_{1}}^{T_{0}}\right| \mathrm{HF}\right\rangle \\
& +\frac{1}{2} \sum_{\mu_{k} v_{m}} \bar{t}_{\mu_{k}}^{(0)} t_{v_{m}}^{X_{j_{2}} X_{j_{3}}}\left(\omega_{X_{j_{2}}}, \omega_{X_{j_{3}}}\right)\left\langle\mu_{k}^{\mathrm{CC}}\left|\left[X_{j_{1}}^{T_{0}}, \tau_{v_{m}}\right]\right| \mathrm{HF}\right\rangle+\sum_{\mu_{k} v_{m}} \bar{t}_{\mu_{k}}^{X_{j_{2}}}\left(\omega_{X_{j_{2}}}\right) t_{v_{m}}^{X_{j_{3}}}\left(\omega_{X_{j_{3}}}\right)\left\langle\mu_{k}^{\mathrm{CC}}\left|\left[X_{j_{1}}^{T_{0}}, \tau_{v_{m}}\right]\right| \mathrm{HF}\right\rangle \\
& \left.+\frac{1}{2} \sum_{\mu_{k} v_{m} \lambda_{n}} \bar{t}_{\mu_{k}}^{(0)} t_{v_{m}}^{X_{j_{2}}}\left(\omega_{X_{j_{2}}}\right) t_{\lambda_{n}}^{X_{j_{3}}}\left(\omega_{X_{j_{3}}}\right)\left\langle\mu_{k}^{\mathrm{CC}}\left|\left[\left[X_{j_{1}}^{T_{0}}, \tau_{v_{m}}\right], \tau_{\lambda_{n}}\right]\right| \mathrm{HF}\right\rangle\right], \quad \omega_{X_{j_{1}}}+\omega_{X_{j_{2}}}+\omega_{X_{j_{3}}}=0 .
\end{aligned}
$$

Using the stationary condition of Eq. (246), we obtain the second-order amplitudes equation,

$$
\begin{aligned}
& \sum_{v_{m}}\left({ }^{\mathrm{CC}} J_{\mu_{k} v_{m}}-\left(\omega_{X_{j_{1}}}+\omega_{X_{j_{2}}}\right) \delta_{\mu_{k} v_{m}}\right) t_{v_{m}}^{\mathrm{X}_{j_{1}} X_{j_{2}}}\left(\omega_{X_{j_{1}}}, \omega_{X_{j_{2}}}\right) \\
& =-\sum_{v_{m} \lambda_{n}}\left\langle\mu_{k}^{\mathrm{CC}}\left|\left[\left[H_{0}^{T_{0}}, \tau_{v_{m}}\right], \tau_{\lambda_{n}}\right]\right| \mathrm{HF}\right\rangle t_{v_{m}}^{X_{j_{1}}}\left(\omega_{X_{j_{1}}}\right) t_{\lambda_{n}}^{X_{j_{2}}}\left(\omega_{X_{j_{2}}}\right) \\
& -P_{\omega_{X_{j_{1}}} \omega_{X_{j_{2}}}}^{X_{j_{1} X_{j_{2}}} \sum_{v_{m}}}\left\langle\mu_{k}^{\mathrm{CC}}\left|\left[X_{j_{1}}^{T_{0}}, \tau_{v_{m}}\right]\right| \mathrm{HF}\right\rangle t_{v_{m}}^{X_{j_{2}}}\left(\omega_{X_{j_{2}}}\right),
\end{aligned}
$$

from which $t_{v_{m}}^{X_{j_{1}} X_{j_{2}}}\left(\omega_{X_{j_{1}}}, \omega_{X_{j_{2}}}\right)$, needed in Subsection VIII E for fourth-order properties, may be calculated.

Using the $2 n+1$ and $2 n+2$ rules, the third-order molecular property expression becomes

$$
\begin{aligned}
& \left\langle\left\langle X_{j_{1}} ; X_{j_{2}}, X_{j_{3}}\right\rangle\right\rangle_{\omega_{X_{j_{2}}, \omega_{X_{j_{3}}}}}
\end{aligned}
$$

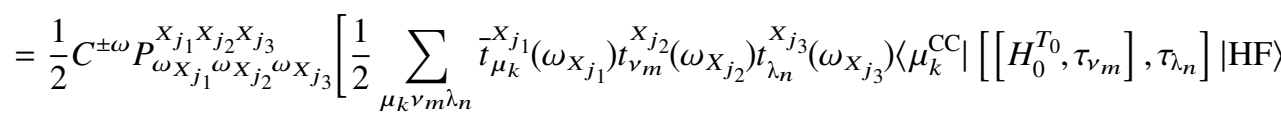

$$
\begin{aligned}
& +\frac{1}{6} \sum_{\mu_{k} \nu_{m} \lambda_{n} \sigma_{p}} \bar{t}_{\mu_{k}}^{(0)} t_{v_{m}}^{X_{j_{1}}}\left(\omega_{X_{j_{1}}}\right) t_{\lambda_{n}}^{X_{j_{2}}}\left(\omega_{X_{j_{2}}}\right) t_{\sigma_{p}}^{X_{j_{3}}}\left(\omega_{X_{j_{3}}}\right)\left\langle\mu_{k}^{\mathrm{CC}}\left|\left[\left[\left[H_{0}^{T_{0}}, \tau_{v_{m}}\right], \tau_{\lambda_{n}}\right], \tau_{\sigma_{p}}\right]\right| \mathrm{HF}\right\rangle \\
& +\sum_{\mu_{k} v_{m}} \bar{t}_{\mu_{k}}^{X_{j_{2}}}\left(\omega_{X_{j_{2}}}\right) t_{v_{m}}^{X_{j_{3}}}\left(\omega_{X_{j_{3}}}\right)\left\langle\mu_{k}^{\mathrm{CC}}\left|\left[X_{j_{1}}^{T_{0}}, \tau_{v_{m}}\right]\right| \mathrm{HF}\right\rangle \\
& \left.+\frac{1}{2} \sum_{\mu_{k} v_{m} \lambda_{n}} \bar{t}_{\mu_{k}}^{(0)} t_{v_{m}}^{X_{j_{2}}}\left(\omega_{X_{j_{2}}}\right) t_{\lambda_{n}}^{X_{j_{3}}}\left(\omega_{X_{j_{3}}}\right)\left\langle\mu_{k}^{\mathrm{CC}}\left|\left[\left[X_{j_{1}}^{T_{0}}, \tau_{v_{m}}\right], \tau_{\lambda_{n}}\right]\right| \mathrm{HF}\right\rangle\right] \quad \omega_{X_{j_{1}}}+\omega_{X_{j_{2}}}+\omega_{X_{j_{3}}}=0
\end{aligned}
$$


where $t_{\mu_{k}}^{(0)}, \bar{t}_{\mu_{k}}^{(0)}, t_{\mu_{k}}^{X_{j_{2}}}\left(\omega_{X_{j_{2}}}\right)$, and $\bar{t}_{\mu_{k}}^{X_{j_{2}}}\left(\omega_{X_{j_{2}}}\right)$ are obtained by solving Eqs. (325), (327), (381), and (382), respectively.

\section{E. Fourth-order molecular properties}

Using the $2 n+1$ and $2 n+2$ rules, we obtain fourth-order molecular properties in terms of the first- and second-order amplitudes and first-order multipliers as

$$
\begin{aligned}
& \left\langle\left\langle X_{j_{1}} ; X_{j_{2}}, X_{j_{3}}, X_{j_{4}}\right\rangle\right\rangle_{\omega_{X_{j_{2}}}, \omega_{X_{j_{3}}}, \omega_{X_{j_{4}}}}
\end{aligned}
$$

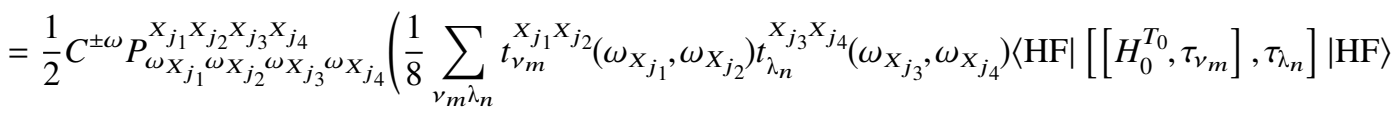

$$
\begin{aligned}
& +\frac{1}{8} \sum_{\mu_{k} v_{m} \lambda_{n}} \bar{t}_{\mu_{k}}^{(0)} t_{v_{m}}^{X_{j_{1}} X_{j_{2}}}\left(\omega_{X_{j_{1}}}, \omega_{X_{j_{2}}}\right) t_{\lambda_{n}}^{X_{j_{3}} X_{j_{4}}}\left(\omega_{X_{j_{3}}}, \omega_{X_{j_{4}}}\right)\left\langle\mu_{k}^{\mathrm{CC}}\left|\left[\left[H_{0}^{T_{0}}, \tau_{v_{m}}\right], \tau_{\lambda_{n}}\right]\right| \mathrm{HF}\right\rangle \\
& +\frac{1}{2} \sum_{\mu_{k} v_{m} \lambda_{n}} \bar{t}_{\mu_{k}}^{X_{j_{1}}}\left(\omega_{X_{j_{1}}}\right) t_{v_{m}}^{X_{j_{2}}}\left(\omega_{X_{j_{2}}}\right) t_{\lambda_{n}}^{X_{j_{3}} X_{j_{4}}}\left(\omega_{X_{j_{3}}}, \omega_{X_{j_{4}}}\right)\left\langle\mu_{k}^{\mathrm{CC}}\left|\left[\left[H_{0}^{T_{0}}, \tau_{v_{m}}\right], \tau_{\lambda_{n}}\right]\right| \mathrm{HF}\right\rangle \\
& +\frac{1}{4} \sum_{\mu_{k} v_{m} \lambda_{n} \sigma_{p}} \bar{t}_{\mu_{k}}^{(0)} t_{v_{m}}^{X_{j_{1}}}\left(\omega_{X_{j_{1}}}\right) t_{\lambda_{n}}^{X_{j_{2}}}\left(\omega_{X_{j_{2}}}\right) t_{\sigma_{p}}^{X_{j_{3}} X_{j_{4}}}\left(\omega_{X_{j_{3}}}, \omega_{X_{j_{4}}}\right)\left\langle\mu_{k}^{\mathrm{CC}}\left|\left[\left[\left[H_{0}^{T_{0}}, \tau_{v_{m}}\right], \tau_{\lambda_{n}}\right], \tau_{\sigma_{p}}\right]\right| \mathrm{HF}\right\rangle \\
& +\frac{1}{6} \sum_{\mu_{k} \nu_{m} \lambda_{n} \sigma_{p}} \bar{t}_{\mu_{k}}^{X_{j_{1}}}\left(\omega_{X_{j_{1}}}\right) t_{v_{m}}^{X_{j_{2}}}\left(\omega_{X_{j_{2}}}\right) t_{\lambda_{n}}^{X_{j_{3}}}\left(\omega_{X_{j_{3}}}\right) t_{\sigma_{p}}^{X_{j_{4}}}\left(\omega_{X_{j_{4}}}\right)\left\langle\mu_{k}^{\mathrm{CC}}\left|\left[\left[\left[H_{0}^{T_{0}}, \tau_{v_{m}}\right], \tau_{\lambda_{n}}\right], \tau_{\sigma_{p}}\right]\right| \mathrm{HF}\right\rangle \\
& +\frac{1}{24} \sum_{\mu_{k} v_{m} \lambda_{n} \sigma_{p} \rho_{q}} \bar{t}_{\mu_{k}}^{(0)} t_{v_{m}}^{X_{j_{1}}}\left(\omega_{X_{j_{1}}}\right) t_{\lambda_{n}}^{X_{j_{2}}}\left(\omega_{X_{j_{2}}}\right) t_{\sigma_{p}}^{X_{j_{3}}}\left(\omega_{X_{j_{3}}}\right) t_{\rho_{q}}^{X_{j_{4}}}\left(\omega_{X_{j_{4}}}\right)\left\langle\mu_{k}^{\mathrm{CC}}\left|\left[\left[\left[\left[H_{0}^{T_{0}}, \tau_{v_{m}}\right], \tau_{\lambda_{n}}\right], \tau_{\sigma_{p}}\right], \tau_{\rho_{q}}\right]\right| \mathrm{HF}\right\rangle \\
& +\frac{1}{2} \sum_{\mu_{k} v_{m}} \bar{t}_{\mu_{k}}^{X_{j_{2}}}\left(\omega_{X_{j_{2}}}\right) t_{v_{m}}^{X_{j_{3}} X_{j_{4}}}\left(\omega_{X_{j_{3}}}, \omega_{X_{j_{4}}}\right)\left\langle\mu_{k}^{\mathrm{CC}}\left|\left[X_{j_{1}}^{T_{0}}, \tau_{v_{m}}\right]\right| \mathrm{HF}\right\rangle \\
& +\frac{1}{2} \sum_{\mu_{k} v_{m} \lambda_{n}} \bar{t}_{\mu_{k}}^{(0)} t_{v_{m}}^{X_{j_{2}}}\left(\omega_{X_{j_{2}}}\right) t_{\lambda_{n}}^{X_{j_{3}} X_{j_{4}}}\left(\omega_{X_{j_{3}}}, \omega_{X_{j_{4}}}\right)\left\langle\mu_{k}^{\mathrm{CC}}\left|\left[\left[X_{j_{1}}^{T_{0}}, \tau_{v_{m}}\right], \tau_{\lambda_{n}}\right]\right| \mathrm{HF}\right\rangle \\
& +\frac{1}{2} \sum_{\mu_{k} v_{m} \lambda_{n}} \bar{t}_{\mu_{k}}^{X_{j_{2}}}\left(\omega_{X_{j_{2}}}\right) t_{v_{m}}^{X_{j_{3}}}\left(\omega_{X_{j_{3}}}\right) t_{\lambda_{n}}^{X_{j_{4}}}\left(\omega_{X_{j_{4}}}\right)\left\langle\mu_{k}^{\mathrm{CC}}\left[\left[\left[X_{j_{1}}^{T_{0}}, \tau_{v_{m}}\right], \tau_{\lambda_{n}}\right]|\mathrm{HF}\rangle\right), \omega_{X_{j_{1}}}+\omega_{X_{j_{2}}}+\omega_{X_{j_{3}}}+\omega_{X_{j_{4}}}=0,\right.
\end{aligned}
$$

where $t_{\mu_{k}}^{(0)}, \bar{t}_{\mu_{k}}^{(0)}, t_{\mu_{k}}^{X_{j_{2}}}\left(\omega_{X_{j_{2}}}\right), \bar{t}_{\mu_{k}}^{X_{j_{2}}}\left(\omega_{X_{j_{2}}}\right)$, and $t_{\mu_{k}}^{X_{j_{2}} X_{j_{3}}}\left(\omega_{X_{j_{2}}}, \omega_{X_{j_{3}}}\right)$ are obtained by solving Eqs. (325), (327), (381), (382), and (385), respectively.

The detailed formulas for the second-, third-, and fourth-order molecular properties in Eqs. (383), (386), and (387), respectively, only include terms where all indices are connected and all matrix elements are fully expressed as commutators. Such an expansion is called connected and is size-extensive also in the more restricted meaning of this term advocated by Bartlett. ${ }^{86}$

\section{F. The CC Jacobian}

The CC and CC-CI Jacobians are equal [Eq. (337)]. However, in the CC model, where the time evolution is exponentially parametrized, the time-evolving state cannot be expanded in the time-independent complete basis $\left\{|\mathrm{HF}\rangle,\left|\mu_{k}^{\mathrm{CC}}\right\rangle\right\}$, as was the case for the linear expansion of the time evolution in the CC-CI model, and we, therefore, cannot obtain an explicit representation for the excited states. However, it is not important that the wave function for the individual states cannot be given an explicit representation, because expressions for experimental observables may be identified from the response functions and their residues without a recourse to an explicit representation of the states.

\section{MOLECULAR PROPERTIES FOR THE EOMCC MODEL}

EOMCC response functions were introduced by Stanton and Bartlett ${ }^{78}$ and Rozyczko and Bartlett ${ }^{79-81}$ and were defined in terms of CI response functions, where the CI energies of Eq. (310) and states of Eqs. (315) and (316) were replaced by the CC energies of Eq. (354) and states of Eqs. (364) and (365). The replacement must be performed for CI response functions that are derived by solving the Hermitian eigenvalue equation via projection, as in Sec. VI, in order to get the operator $\frac{1}{2} C^{ \pm \omega}$ introduced in the EOMCC response functions. 
In the following, we derive the first-, second-, and thirdorder molecular property expressions for the EOMCC model. First-order properties are straightforwardly obtained from Eq. (293) as

$$
\left\langle\left\langle X_{j_{1}}\right\rangle\right\rangle_{0}^{\mathrm{EOMCC}}=\frac{1}{2} C^{ \pm \omega}\left\langle 0_{0}^{\mathrm{CC}}\left|X_{j_{1}}\right| 0_{0}^{\mathrm{CC}}\right\rangle,
$$

where the $\left|0_{0}^{\mathrm{CC}}\right\rangle$ and $\left\langle 0_{0}^{\mathrm{CC}}\right|$ are given in Eqs. (366) and (367), respectively, and therefore,

$$
\operatorname{EOMCC}_{b}^{(0)}=\bar{t}_{\mu_{k}}^{(0)} .
$$

The CI second- and third-order molecular property expressions may be expressed in the diagonal representation as

$$
\begin{aligned}
& \left\langle\left\langle X_{j_{1}} ; X_{j_{2}}\right\rangle\right\rangle_{\omega_{X_{j_{2}}}}^{\mathrm{CI}}=\frac{1}{2} C^{ \pm \omega} P_{\omega_{X_{j_{1}}} \omega_{X_{j_{2}}}}^{X_{j_{1} X_{j_{2}}}} \sum_{k}\left\langle 0_{0}^{\mathrm{CI}}\left|X_{j_{1}}\right| 0_{k}^{\mathrm{CI}}\right\rangle \\
& \times{ }^{\mathrm{CI}} s_{k}^{X_{j_{2}}}\left(\omega_{X_{j_{2}}}\right), \quad \omega_{X_{j_{1}}}+\omega_{X_{j_{2}}}=0, \\
& \left\langle\left\langle X_{j_{1}} ; X_{j_{2}}, X_{j_{3}}\right\rangle\right\rangle_{\omega_{X_{j_{2}}}, \omega_{X_{j_{3}}}}^{\mathrm{CI}}
\end{aligned}
$$

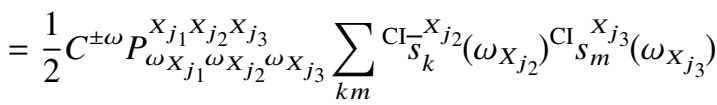

$$
\begin{aligned}
& \times\left(\left\langle 0_{k}^{\mathrm{CI}}\left|X_{j_{1}}\right| 0_{m}^{\mathrm{CI}}\right\rangle-\left\langle 0_{0}^{\mathrm{CI}}\left|X_{j_{1}}\right| 0_{0}^{\mathrm{CI}}\right\rangle \delta_{k m}\right), \\
& \omega_{X_{j_{1}}}+\omega_{X_{j_{2}}}+\omega_{X_{j_{3}}}=0 \text {, }
\end{aligned}
$$

where ${ }^{\mathrm{Cl}} s_{k}^{X_{j_{3}}}\left(\omega_{X_{j_{3}}}\right)$ and ${ }^{\mathrm{Cl}} \bar{s}_{k}^{X_{j_{2}}}\left(\omega_{X_{j_{2}}}\right)$ denote the coefficients and multipliers in the basis of the eigenstates of $H_{0}$,

$$
\begin{aligned}
& { }^{\mathrm{CI}} s_{n}^{{ }^{X}{ }_{j_{1}}}\left(\omega_{X_{j_{1}}}\right)=\sum_{\mu_{k}}{ }^{\mathrm{CC}} \bar{C}_{n \mu_{k}}{ }^{\mathrm{CI}} s_{\mu_{k}}^{X_{j_{1}}}\left(\omega_{X_{j_{1}}}\right) \text {, } \\
& \mathrm{CI}_{\bar{s}}^{X_{n}}{ }_{j_{1}}\left(\omega_{X_{j_{1}}}\right)=\sum_{\mu_{k}} \mathrm{CI}_{\bar{s}_{\mu_{k}}}^{X_{j_{1}}}\left(\omega_{X_{j_{1}}}\right){ }^{\mathrm{CC}} C_{\mu_{k} n}
\end{aligned}
$$

and may be obtained from the first-order response equations

$$
\begin{aligned}
\left({ }^{\mathrm{CI}} E_{k 0}-\omega_{X_{j_{1}}}\right){ }^{\mathrm{CI}} s_{k}^{X_{j_{1}}}\left(\omega_{X_{j_{1}}}\right) & =-\left\langle 0_{k}^{\mathrm{CI}}\left|X_{j_{1}}\right| 0_{0}^{\mathrm{CI}}\right\rangle, \\
{ }^{\mathrm{CI}}{ }_{k}{ }_{k}^{X_{j_{1}}}\left(\omega_{X_{j_{1}}}\right)\left({ }^{\mathrm{CI}} E_{k 0}+\omega_{X_{j_{1}}}\right) & =-\left\langle 0_{0}^{\mathrm{CI}}\left|X_{j_{1}}\right| 0_{k}^{\mathrm{CI}}\right\rangle .
\end{aligned}
$$

The EOMCC second- and third-order molecular properties are obtained by replacing in Eqs. (390) and (391) and Eqs. (394) and (395) the CI eigenstates and eigenvalues of Eqs. (315), (316), and (321) by the CC eigenstates and eigenvalues of Eqs. (364), (365), and (373),

$$
\begin{aligned}
& \left\langle\left\langle X_{j_{1}} ; X_{j_{2}}\right\rangle\right\rangle \omega_{\omega_{X_{j_{2}}}}^{\mathrm{EOMCC}} \\
& =\frac{1}{2} C^{ \pm \omega} P_{\omega_{X_{j_{1}}} \omega_{X_{j_{2}}}}^{X_{j_{1}} X_{j_{2}}} \sum_{k}\left\langle 0_{0}^{\mathrm{CC}}\left|X_{j_{1}}\right| 0_{k}^{\mathrm{CC}}\right\rangle{ }^{\mathrm{EOMCC}} b_{k}^{X_{j_{2}}}\left(\omega_{X_{j_{2}}}\right), \\
& \omega_{X_{j_{1}}}+\omega_{X_{j_{2}}}=0
\end{aligned}
$$

$$
\begin{aligned}
\left\langle\left\langle X_{j_{1}} ;\right.\right. & \left.\left.X_{j_{2}}, X_{j_{3}}\right\rangle\right\rangle_{\omega_{X_{j_{2}}} \omega_{X_{j_{3}}}}^{\mathrm{EOMCC}} \\
= & \frac{1}{2} C^{ \pm \omega} P_{\omega_{X_{j_{1}}} \omega_{X_{j_{2}}} \mathrm{X}_{j_{j_{2}} X_{j_{2}} X_{j_{3}}} \omega_{j_{3}}} \sum_{k m}^{\mathrm{EOMCC}} b_{k}^{X_{j_{2}}}\left(\omega_{X_{j_{2}}}\right) \\
& \times\left(\left\langle 0_{k}^{\mathrm{CC}}\left|X_{j_{1}}\right| 0_{m}^{\mathrm{CC}}\right\rangle-\left\langle 0_{0}^{\mathrm{CC}}\left|X_{j_{1}}\right| 0_{0}^{\mathrm{CC}}\right\rangle \delta_{k m}\right){ }^{\mathrm{EOMCC}} b_{m}^{X_{j_{3}}}\left(\omega_{X_{j_{3}}}\right), \\
& \omega_{X_{j_{1}}}+\omega_{X_{j_{2}}}+\omega_{X_{j_{3}}}=0,
\end{aligned}
$$

where ${ }^{\operatorname{EOMCC}_{b}} b_{k}^{X_{j_{3}}}\left(\omega_{X_{j_{3}}}\right)$ and ${ }^{\text {EOMCC }} b_{k}^{X_{j_{2}}}\left(\omega_{X_{j_{2}}}\right)$ are the solutions to the first-order EOMCC right and left response equations in the diagonal representation,

$$
\left({ }^{\mathrm{CC}} E_{k 0}-\omega_{X_{j_{1}}}\right){ }^{\text {EOMCC }} b_{k}^{X_{j_{1}}}\left(\omega_{X_{j_{1}}}\right)=-\left\langle 0_{k}^{\mathrm{CC}}\left|X_{j_{1}}\right| 0_{0}^{\mathrm{CC}}\right\rangle,
$$

$\mathrm{EOMCC}_{b}^{-X_{j_{1}}}\left(\omega_{X_{j_{1}}}\right)\left({ }^{\mathrm{CC}} E_{k 0}+\omega_{X_{j_{1}}}\right)=-\left\langle 0_{0}^{\mathrm{CC}}\left|X_{j_{1}}\right| 0_{k}^{\mathrm{CC}}\right\rangle$.

The response functions may be obtained in the nondiagonal representation by transforming Eqs. (396) and (397) back to the basis of Eqs. (332) and (345), using Eqs. (363) and (366)-(369). The back transformation may be expressed as

$$
\begin{gathered}
\operatorname{EOMCC}_{\mu_{k}}^{X_{j_{1}}}\left(\omega_{X_{j_{1}}}\right)=\sum_{n}{ }^{\mathrm{CC}} C_{\mu_{k} n}{ }^{\text {EOMCC }} b_{n}^{X_{j_{1}}}\left(\omega_{X_{j_{1}}}\right), \\
{ }^{\text {EOMCC }} b_{\mu_{k}}^{X_{j_{1}}}\left(\omega_{X_{j_{1}}}\right)=\sum_{n}{ }^{\mathrm{EOMCC}} b_{n}^{X_{j_{1}}}\left(\omega_{X_{j_{1}}}\right)^{\mathrm{CC}} \bar{C}_{n \mu_{k}}
\end{gathered}
$$

and gives the first-order amplitude equations [Eq. (398)] in the non-diagonal representation as

$$
\begin{gathered}
\sum_{v_{m}}\left({ }^{\mathrm{CC}} J_{\mu_{k} v_{m}}-\omega_{X_{j_{1}}} \delta_{\mu_{k} v_{m}}\right){ }^{\text {EOMCC }} b_{v_{m}}^{X_{j_{1}}}\left(\omega_{X_{j_{1}}}\right) \\
=-\left\langle\mu_{k}^{\mathrm{CC}}\left|X_{j_{1}}^{T_{0}}\right| \mathrm{HF}\right\rangle,
\end{gathered}
$$

which is identical to Eqs. (338) and (381), and therefore, the first-order wave function coefficients in the CC-CI and EOMCC models and the first-order amplitudes in the CC model are equal,

$$
{ }^{\operatorname{EOMCC}} b_{\mu_{k}}^{X_{j_{1}}}\left(\omega_{X_{j_{1}}}\right)={ }^{\mathrm{CC}-\mathrm{CI}}{ }_{\mu_{\mu_{k}}}^{X_{j_{1}}}\left(\omega_{X_{j_{1}}}\right)=t_{\mu_{k}}^{X_{j_{1}}}\left(\omega_{X_{j_{1}}}\right) .
$$

For the first-order Lagrangian multipliers of Eq. (399), the back transformation in Eq. (401) gives

$$
\begin{aligned}
\sum_{\mu_{k}}{ }^{\mathrm{EOMCC}} \bar{b}_{\mu_{k}}^{X_{j_{1}}}\left(\omega_{X_{j_{1}}}\right)\left({ }^{\mathrm{CC}} J_{\mu_{k} v_{m}}+\omega_{X_{j_{1}}} \delta_{\mu_{k} v_{m}}\right) \\
=-\left\langle\mathrm{HF}\left|X_{j_{1}}^{T_{0}}\right| v_{m}^{\mathrm{CC}}\right\rangle+\bar{t}_{v_{m}}^{(0)}\left\langle\mathrm{HF}\left|X_{j_{1}}^{T_{0}}\right| \mathrm{HF}\right\rangle \\
-\sum_{\mu_{k}} \bar{t}_{\mu_{k}}^{(0)}\left\langle\mu_{k}^{\mathrm{CC}}\left|X_{j_{1}}^{T_{0}}\right| v_{m}^{\mathrm{CC}}\right\rangle \\
+\bar{t}_{v_{m}}^{(0)} \sum_{\mu_{k}} \bar{t}_{\mu_{k}}^{(0)}\left\langle\mu_{k}^{\mathrm{CC}}\left|X_{j_{1}}^{T_{0}}\right| \mathrm{HF}\right\rangle
\end{aligned}
$$

which is clearly different from Eqs. (339) and (382),

$$
\operatorname{EOMCC}^{-} \bar{b}_{\mu_{k}}^{X_{j_{1}}}\left(\omega_{X_{j_{1}}}\right) \neq{ }^{\mathrm{CC}-\mathrm{CI}}-\bar{s}_{\mu_{k}}^{X_{j_{1}}}\left(\omega_{X_{j_{1}}}\right),
$$




$$
\operatorname{EOMCC}^{-} \bar{b}_{\mu_{k}}^{X_{j_{1}}}\left(\omega_{X_{j_{1}}}\right) \neq \bar{t}_{\mu_{k}}^{X_{j_{1}}}\left(\omega_{X_{j_{1}}}\right) .
$$

The EOMCC second- and third-order molecular property expressions become

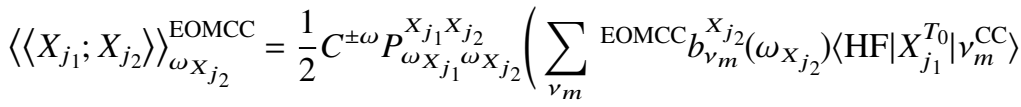

$$
\begin{aligned}
& -\left(\sum_{v_{m}} \bar{t}_{v_{m}}^{(0)}{ }^{\mathrm{EOMCC}} b_{v_{m}}^{X_{j_{2}}}\left(\omega_{X_{j_{2}}}\right)\right)\left\langle\mathrm{HF}\left|X_{j_{1}}^{T_{0}}\right| \mathrm{HF}\right\rangle+\sum_{\mu_{k} v_{m}} \bar{t}_{\mu_{k}}^{(0)} \mathrm{EOMCC}_{b_{v_{m}}}^{X_{j_{2}}}\left(\omega_{X_{j_{2}}}\right)\left\langle\mu_{k}^{\mathrm{CC}}\left|X_{j_{1}}^{T_{0}}\right| v_{m}^{\mathrm{CC}}\right\rangle \\
& \left.-\sum_{v_{m}} \bar{t}_{v_{m}}^{(0)} \operatorname{EOMCC}_{b_{v_{m}}}^{X_{j_{2}}}\left(\omega_{X_{j_{2}}}\right) \sum_{\mu_{k}} \bar{t}_{\mu_{k}}^{(0)}\left\langle\mu_{k}^{\mathrm{CC}}\left|X_{j_{1}}^{T_{0}}\right| \mathrm{HF}\right\rangle\right), \quad \omega_{X_{j_{1}}}+\omega_{X_{j_{2}}}=0,
\end{aligned}
$$

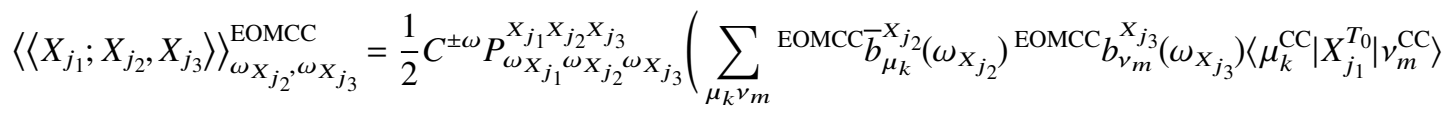

$$
\begin{aligned}
& -\left(\sum_{v_{m}} \bar{t}_{v_{m}}^{(0)}{ }^{\mathrm{EOMCC}} b_{v_{m}}^{X_{j_{3}}}\left(\omega_{X_{j_{3}}}\right)\right) \sum_{\mu_{k}}{ }^{\mathrm{EOMCC}} \bar{b}_{\mu_{k}}^{X_{j_{2}}}\left(\omega_{X_{j_{2}}}\right)\left\langle\mu_{k}^{\mathrm{CC}}\left|X_{j_{1}}^{T_{0}}\right| \mathrm{HF}\right\rangle \\
& -\left(\sum_{v_{m}}{ }^{\mathrm{EOMCC}} \bar{b}_{v_{m}}^{X_{j_{2}}}\left(\omega_{X_{j_{2}}}\right)^{\mathrm{EOMCC}} b_{v_{m}}^{X_{j_{3}}}\left(\omega_{X_{j_{3}}}\right)\right)\left\langle\mathrm{HF}\left|X_{j_{1}}^{T_{0}}\right| \mathrm{HF}\right\rangle \\
& \left.-\left(\sum_{v_{m}}{ }^{\mathrm{EOMCC}} b_{v_{m}}^{X_{j_{2}}}\left(\omega_{X_{j_{2}}}\right){ }^{\mathrm{EOMCC}} b_{v_{m}}^{X_{j_{3}}}\left(\omega_{X_{j_{3}}}\right)\right) \sum_{\mu_{k}} \bar{t}_{\mu_{k}}^{(0)}\left\langle\mu_{k}^{\mathrm{CC}}\left|X_{j_{1}}^{T_{0}}\right| \mathrm{HF}\right\rangle\right), \\
& \omega_{X_{j_{1}}}+\omega_{X_{j_{2}}}+\omega_{X_{j_{3}}}=0,
\end{aligned}
$$

where $t_{\mu_{k}}^{(0)}$ and $\bar{t}_{\mu_{k}}^{(0)}$ are obtained by solving Eqs. (325) and (327), respectively, while ${ }^{\mathrm{EOMCC}} b_{\mu_{k}}^{X_{j_{3}}}\left(\omega_{X_{j_{3}}}\right)$ and $\mathrm{EOMCC}^{-X_{j_{2}}}$ $\left(\omega_{X_{j_{2}}}\right)$ are obtained from Eqs. (402) and (404).

\section{COMPARISON OF THE Cl, CC-CI, CC, AND EOMCC MOLECULAR RESPONSE PROPERTIES}

In Sec. X A, we discuss how static molecular properties may be obtained from finite field energy calculations. In particular, we describe how the finite field method can be generalized to be applicable for the CC-CI model. In Sec. $\mathrm{X} \mathrm{B}$, the size-extensivity of molecular properties is discussed for the CI, CC-CI, and CC models. In Sec. X C, the focus is on the different characteristics and properties of response functions when a linear versus an exponential parametrization is considered for the unperturbed state and its time evolution. In Sec. X D, a comparison is performed of $\mathrm{CC}-\mathrm{CI}$ and EOMCC response functions.

\section{A. Static molecular properties from finite field energy calculations in the $\mathrm{Cl}, \mathrm{CC}-\mathrm{Cl}$ and $\mathrm{CC}$ models}

We have seen in Sec. II D that in the limit of a timeindependent perturbation, where the frequencies in Eq. (3) are zero, the perturbed Hamiltonian becomes time-independent

$$
H(\epsilon)=H_{0}+V(\epsilon)
$$

The quasi-energy, $\mathcal{E}(\epsilon)$, then becomes the energy, $E_{0}(\epsilon)$ [Eq. (50)], of the eigenvalue equation for $H(\epsilon)$ [Eq. (49)],

$$
\mathcal{E}(\epsilon) \longrightarrow E_{0}(\epsilon)
$$

and the regular wave function, $\left|0_{R}(t, \epsilon)\right\rangle$, becomes the eigenstate $\left|0_{0}(\epsilon)\right\rangle$,

$$
\left|0_{R}(t, \epsilon)\right\rangle \longrightarrow\left|0_{0}(\epsilon)\right\rangle
$$

For any wave function model, in which a time-dependent phase is isolated giving the regular wave function [see Sec. II B], the perturbed energy, $E_{0}(\epsilon)$, may therefore be used in finite field energy calculations to obtain static molecular properties that are equal to static limit response function results.

\section{Standard finite field energy calculations of static molecular properties}

The standard way of performing finite field energy calculations for determination of static molecular properties is to include the static perturbation operator in the timeindependent Hamiltonian $H(\epsilon)$ [Eq. (409)] and optimize the energy for this perturbed Hamiltonian. The optimization may, for example, be done using the variational principle [Eq. (57)]

$$
\left\langle\delta 0_{0}(\epsilon)|H(\epsilon)| 0_{0}(\epsilon)\right\rangle+\left\langle 0_{0}(\epsilon)|H(\epsilon)| \delta 0_{0}(\epsilon)\right\rangle=0
$$


and the optimized perturbed energy $E_{0}(\epsilon)$ [Eq. (56)] then becomes

$$
E_{0}(\epsilon)=\left\langle 0_{0}(\epsilon)|H(\epsilon)| 0_{0}(\epsilon)\right\rangle .
$$

Differentiation of $E_{0}(\epsilon)$ with respect to the perturbation strengths, $\epsilon$, then yields static molecular properties. When the finite field method defined by Eqs. (412) and (413) is used to determine static molecular properties, we obtain properties that are equal to the static limit response function properties of Sec. III, as in Sec. III, we have assumed that both the unperturbed state and its time evolution are determined using the variational principle.

The standard finite field energy method may, of course, also be applied when the variational principle is replaced by projection. This is, for example, done in CC theory [Sec. VIII] where projection is used to obtain an optimized $\mathrm{CC}$ wave function both for the unperturbed and the perturbed system.

In this article, we have considered more general wave function models, where the unperturbed state and its time evolution are determined using different parametrizations and where different optimization conditions have been applied for the unperturbed and perturbed system. To obtain static molecular properties from finite field calculations for such models, the above described finite field method has to be generalized. In Sec. X A 2, we describe a finite field approach for obtaining static limit CC-CI response function results, and in Sec. X A 3, we show that these static limit properties may also be obtained from standard finite field EOMCC energy calculations.

\section{Finite field energy calculations for the $\mathrm{CC}-\mathrm{Cl}$ model}

We now describe a finite field energy method for determining static CC-CI molecular properties. In the static limit, the CC-CI intermediate-normalized wave function [Eq. (331)] becomes

$$
\begin{aligned}
\left|0_{I}^{\mathrm{CC}-\mathrm{CI}}(\epsilon)\right\rangle & =\exp \left(T_{0}\right) \exp (S(\epsilon))|\mathrm{HF}\rangle \\
& =\exp \left(T_{0}\right)|\mathrm{HF}\rangle+\sum_{\mu_{k}} s_{\mu_{k}}(\epsilon) \exp \left(T_{0}\right)\left|\mu_{k}^{\mathrm{CC}}\right\rangle,
\end{aligned}
$$

where the cluster amplitudes, $t_{\mu_{k}}^{(0)}$, entering the operator $T_{0}$, are determined from the $\mathrm{CC}$ amplitudes equations [Eq. (325)]. Within the CC-CI framework, the energy of the CC-CI wave function in Eq. (414) is obtained as the static limit equivalent of Eq. (206a), whereas the amplitudes $s_{\mu_{k}}(\epsilon)$ are obtained from the static limit equivalent of Eq. (206c).

The CC-CI perturbed energy thus may be obtained from the quasi-energy expression in Eq. (206a) as

$$
\begin{aligned}
{ }^{\mathrm{CC}-\mathrm{Cl}} E(\epsilon)= & \left\langle\mathrm{HF}\left|\exp (-S(\epsilon))\left(H_{0}^{T_{0}}+V^{T_{0}}(\epsilon)\right) \exp (S(\epsilon))\right| \mathrm{HF}\right\rangle \\
= & \left\langle\mathrm{HF}\left|(1-S(\epsilon))\left(H_{0}^{T_{0}}+V^{T_{0}}(\epsilon)\right)(1+S(\epsilon))\right| \mathrm{HF}\right\rangle \\
= & { }^{\mathrm{CC}} E_{0}+\sum_{\mu_{k}}\left\langle\mathrm{HF}\left|H_{0}^{T_{0}}\right| \mu_{k}^{\mathrm{CC}}\right\rangle s_{\mu_{k}}(\epsilon)+\langle\mathrm{HF}| V^{T_{0}}(\epsilon) \\
& \times|\mathrm{HF}\rangle+\sum_{\mu_{k}}\left\langle\mathrm{HF}\left|V^{T_{0}}(\epsilon)\right| \mu_{k}^{\mathrm{CC}}\right\rangle_{\mu_{k}}(\epsilon),
\end{aligned}
$$

where the set of perturbed amplitudes, $s_{\mu_{k}}(\epsilon)$, is determined from the static limit of the amplitude equations in Eq. (206c),

$$
\left\langle\mu_{k}^{\mathrm{CC}}\left|\exp (-S(\epsilon))\left(H_{0}^{T_{0}}+V^{T_{0}}(\epsilon)\right) \exp (S(\epsilon))\right| \mathrm{HF}\right\rangle=0 .
$$

To simplify the perturbed amplitude equations, consider the first term on the left-hand side of Eq. (416),

$$
\begin{aligned}
\left\langle\mu_{k}^{\mathrm{CC}}\right| & \exp (-S(\epsilon)) H_{0}^{T_{0}} \exp (S(\epsilon))|\mathrm{HF}\rangle \\
= & \left\langle\mu_{k}^{\mathrm{CC}}\left|(1-S(\epsilon)) H_{0}^{T_{0}}(1+S(\epsilon))\right| \mathrm{HF}\right\rangle \\
= & \left\langle\mu_{k}^{\mathrm{CC}}\left|H_{0}^{T_{0}}\right| \mathrm{HF}\right\rangle \\
& +\sum_{v_{m}}\left(\left\langle\mu_{k}^{\mathrm{CC}}\left|H_{0}^{T_{0}}\right| v_{m}^{\mathrm{CC}}\right\rangle-\delta_{\mu_{k} \nu_{m}}\left\langle\mathrm{HF}\left|H_{0}^{T_{0}}\right| \mathrm{HF}\right\rangle\right) s_{v_{m}}(\epsilon) \\
& -s_{\mu_{k}}(\epsilon) \sum_{v_{m}}\left\langle\mathrm{HF}\left|H_{0}^{T_{0}}\right| v_{m}^{\mathrm{CC}}\right\rangle_{v_{v_{m}}}(\epsilon) \\
= & \sum_{v_{m}}{ }^{\mathrm{CC}} J_{\mu_{k} v_{m}} s_{v_{m}}(\epsilon)-s_{\mu_{k}}(\epsilon) \sum_{v_{m}}\left\langle\mathrm{HF}\left|H_{0}^{T_{0}}\right| v_{m}^{\mathrm{CC}}\right\rangle_{v_{\nu_{m}}}(\epsilon),
\end{aligned}
$$

where to obtain the last equality we have used the definition of the CC Jacobian [Eq. (328)]. Using Eq. (417), we may write Eq. (416) as

$$
\begin{aligned}
& \sum_{v_{m}}{ }^{\mathrm{CC}} J_{\mu_{k} v_{m}} s_{v_{m}}(\epsilon)-s_{\mu_{k}}(\epsilon) \sum_{v_{m}}\left\langle\mathrm{HF}\left|H_{0}^{T_{0}}\right| v_{m}^{\mathrm{CC}}\right\rangle_{v_{m}}(\epsilon) \\
& \quad+\sum_{v_{m}}\left(\left\langle\mu_{k}^{\mathrm{CC}}\left|V^{T_{0}}(\epsilon)\right| v_{m}^{\mathrm{CC}}\right\rangle-\delta_{\mu_{k} v_{m}}\left\langle\mathrm{HF}\left|V^{T_{0}}(\epsilon)\right| \mathrm{HF}\right\rangle\right) s_{v_{m}}(\epsilon) \\
& \quad-s_{\mu_{k}}(\epsilon) \sum_{v_{m}}\left\langle\mathrm{HF}\left|V^{T_{0}}(\epsilon)\right| v_{m}^{\mathrm{CC}}\right\rangle_{v_{m}}(\epsilon) \\
& \quad+\left\langle\mu_{k}^{\mathrm{CC}}\left|V^{T_{0}}(\epsilon)\right| \mathrm{HF}\right\rangle=0 .
\end{aligned}
$$

Differentiating the energy of Eq. (415) with respect to perturbation strengths, $\epsilon$, yields the static CC-CI molecular properties.

\section{Static $\mathrm{CC}$ - $\mathrm{Cl}$ molecular properties from finite field EOMCC energy calculations}

In Subsection X A 2, we have described how CCCI static molecular properties may be determined from finite field energy calculations, where the perturbed system is described by the CC-CI intermediate-normalized wave function $\left|0_{I}^{\mathrm{CC}-\mathrm{CI}}(\epsilon)\right\rangle$ of Eq. (414). The determination of $\left|0_{I}^{\mathrm{CC}-\mathrm{CI}}(\epsilon)\right\rangle$ in Eq. (414) is performed in the biorthonormal basis $\left|\mathcal{B}^{T_{0}}\right\rangle$ [Eq. (332)] and $\left\langle\mathcal{B}^{T_{0}}\right|$ [Eq. (345)], which is the basis that is used to obtain the EOMCC eigenvalue equation in Eqs. (346)-(354). We now show that static CCCI molecular properties may also be obtained from finite field EOMCC energy calculations.

The EOMCC right ground state eigenvalue equation in Eq. (346) for the perturbed Hamiltonian, $H(\epsilon)$ [Eq. (409)], may be written as

$$
\mathbf{H}^{T_{0}}(\epsilon) \mathbf{C}_{0}^{\mathrm{R}}(\epsilon)=\mathbf{C}_{0}^{\mathrm{R}}(\epsilon) E_{0}(\epsilon),
$$


where the matrix elements of the perturbed Hamiltonian matrix, $\mathbf{H}^{T_{0}}(\epsilon)$, are given by

$$
\begin{aligned}
{\left[\mathbf{H}^{T_{0}}(\epsilon)\right]_{\mathrm{HF} \mathrm{HF}} } & =\left\langle\mathrm{HF}\left|\exp \left(-T_{0}\right)\left(H_{0}+V(\epsilon)\right) \exp \left(T_{0}\right)\right| \mathrm{HF}\right\rangle \\
& ={ }^{\mathrm{CC}} E_{0}+\left\langle\mathrm{HF}\left|V^{T_{0}}(\epsilon)\right| \mathrm{HF}\right\rangle, \\
{\left[\mathbf{H}^{T_{0}}(\epsilon)\right]_{\mathrm{HF} v_{m}} } & =\left\langle\mathrm{HF}\left|\exp \left(-T_{0}\right)\left(H_{0}+V(\epsilon)\right) \exp \left(T_{0}\right)\right| v_{m}^{\mathrm{CC}}\right\rangle \\
& =\left\langle\mathrm{HF}\left|H_{0}^{T_{0}}\right| v_{m}^{\mathrm{CC}}\right\rangle+\left\langle\mathrm{HF}\left|V^{T_{0}}(\epsilon)\right| v_{m}^{\mathrm{CC}}\right\rangle, \\
{\left[\mathbf{H}^{T_{0}}(\epsilon)\right]_{\mu_{k} \mathrm{HF}} } & =\left\langle\mu_{k}^{\mathrm{CC}}\left|\exp \left(-T_{0}\right)\left(H_{0}+V(\epsilon)\right) \exp \left(T_{0}\right)\right| \mathrm{HF}\right\rangle \\
& =\left\langle\mu_{k}^{\mathrm{CC}}\left|V^{T_{0}}(\epsilon)\right| \mathrm{HF}\right\rangle,
\end{aligned}
$$

$$
\begin{aligned}
{\left[\mathbf{H}^{T_{0}}(\epsilon)\right]_{\mu_{k} v_{m}} } & =\left\langle\mu_{k}^{\mathrm{CC}}\left|\exp \left(-T_{0}\right)\left(H_{0}+V(\epsilon)\right) \exp \left(T_{0}\right)\right| v_{m}^{\mathrm{CC}}\right\rangle \\
& =\left\langle\mu_{k}^{\mathrm{CC}}\left|H_{0}^{T_{0}}\right| v_{m}^{\mathrm{CC}}\right\rangle+\left\langle\mu_{k}^{\mathrm{CC}}\left|V^{T_{0}}(\epsilon)\right| v_{m}^{\mathrm{CC}}\right\rangle .
\end{aligned}
$$

To obtain Eq. (422), we have used that the cluster amplitudes equation [Eq. (325)] is satisfied for the unperturbed system. We will assume that the right ground state eigenvector, $\mathbf{C}_{0}^{\mathrm{R}}(\epsilon)$, is intermediate normalized

$$
\mathbf{C}_{0}^{\mathrm{R}}(\epsilon)=\left(\begin{array}{c}
1 \\
c_{\mu_{k}}(\epsilon)
\end{array}\right),
$$

as is the CC-CI ground state wave function [Eq. (414)]. Inserting Eqs. (420)-(424) in Eq. (419), we obtain

$$
\left(\begin{array}{cc}
\mathrm{CC}^{E_{0}}+\left\langle\mathrm{HF}\left|V^{T_{0}}(\epsilon)\right| \mathrm{HF}\right\rangle & \left\langle\mathrm{HF}\left|H_{0}^{T_{0}}\right| v_{m}^{\mathrm{CC}}\right\rangle+\left\langle\mathrm{HF}\left|V^{T_{0}}(\epsilon)\right| v_{m}^{\mathrm{CC}}\right\rangle \\
\left\langle\mu_{k}^{\mathrm{CC}}\left|V^{T_{0}}(\epsilon)\right| \mathrm{HF}\right\rangle & \left\langle\mu_{k}^{\mathrm{CC}}\left|H_{0}^{T_{0}}\right| v_{m}^{\mathrm{CC}}\right\rangle+\left\langle\mu_{k}^{\mathrm{CC}}\left|V^{T_{0}}(\epsilon)\right| v_{m}^{\mathrm{CC}}\right\rangle
\end{array}\right)\left(\begin{array}{c}
1 \\
c_{v_{m}}(\epsilon)
\end{array}\right)=\left(\begin{array}{c}
1 \\
c_{\mu_{k}}(\epsilon)
\end{array}\right) E_{0}(\epsilon),
$$

which gives the perturbed energy and amplitude equations

$$
\begin{aligned}
& E_{0}(\epsilon)={ }^{\mathrm{CC}} E_{0}+\left\langle\mathrm{HF}\left|V^{T_{0}}(\epsilon)\right| \mathrm{HF}\right\rangle+\sum_{v_{m}}\left\langle\mathrm{HF}\left|H_{0}^{T_{0}}\right| v_{m}^{\mathrm{CC}}\right\rangle c_{v_{m}}(\epsilon) \\
&+\sum_{v_{m}}\left\langle\mathrm{HF}\left|V^{T_{0}}(\epsilon)\right| v_{m}^{\mathrm{CC}}\right\rangle c_{v_{m}}(\epsilon), \\
&\left\langle\mu_{k}^{\mathrm{CC}}\left|V^{T_{0}}(\epsilon)\right| \mathrm{HF}\right\rangle+\sum_{v_{m}}\left\langle\mu_{k}^{\mathrm{CC}}\left|H_{0}^{T_{0}}\right| v_{m}^{\mathrm{CC}}\right\rangle c_{v_{m}}(\epsilon) \\
&+\sum_{v_{m}}\left\langle\mu_{k}^{\mathrm{CC}}\left|V^{T_{0}}(\epsilon)\right| v_{m}^{\mathrm{CC}}\right\rangle c_{v_{m}}(\epsilon)-c_{\mu_{k}}(\epsilon) E_{0}(\epsilon)=0 .
\end{aligned}
$$

Substituting Eq. (426) in Eq. (427) and using Eq. (328) gives, after some rearrangements,

$$
\begin{aligned}
\left\langle\mu_{k}^{\mathrm{CC}}\left|V^{T_{0}}(\epsilon)\right| \mathrm{HF}\right\rangle+\sum_{v_{m}}{ }^{\mathrm{CC}} J_{\mu_{k} v_{m}} c_{v_{m}}(\epsilon) \\
\quad+\sum_{v_{m}}\left(\left\langle\mu_{k}^{\mathrm{CC}}\left|V^{T_{0}}(\epsilon)\right| v_{m}^{\mathrm{CC}}\right\rangle-\delta_{\mu_{k} v_{m}}\right. \\
\left.\quad \times\left\langle\mathrm{HF}\left|V^{T_{0}}(\epsilon)\right| \mathrm{HF}\right\rangle\right) c_{v_{m}}(\epsilon) \\
\quad-c_{\mu_{k}}(\epsilon) \sum_{v_{m}}\left\langle\mathrm{HF}\left|H_{0}^{T_{0}}\right| v_{m}^{\mathrm{CC}}\right\rangle c_{v_{m}}(\epsilon) \\
\quad-c_{\mu_{k}}(\epsilon) \sum_{v_{m}}\left\langle\mathrm{HF}\left|V^{T_{0}}(\epsilon)\right| v_{m}^{\mathrm{CC}}\right\rangle c_{v_{m}}(\epsilon)=0 .
\end{aligned}
$$

It follows from Eqs. (418) and (428) that

$$
c_{\mu_{k}}(\epsilon)=s_{\mu_{k}}(\epsilon),
$$

for all $\mu_{k}$, and therefore, the EOMCC perturbed energy ${ }^{\text {EOMCC }} E_{0}(\epsilon)$ [Eq. (426)] is equal to the CC-CI perturbed energy [Eq. (415)],

$$
{ }^{\text {EOMCC }} E_{0}(\epsilon)={ }^{\mathrm{CC}-\mathrm{Cl}} E(\epsilon) .
$$

CC-CI static molecular properties may therefore be obtained by differentiation of the EOMCC energy in Eq. (426). If the EOMCC eigenvalue equation [Eq. (419)] is solved for excited state energies, we obtain static molecular properties for the excited states that are equal to the CC-CI excited state static molecular properties.

\section{B. Size-extensivity of molecular properties in the $\mathrm{Cl}$, $\mathrm{CC}-\mathrm{Cl}$, and $\mathrm{CC}$ models}

In Sec. II G, we have seen that a multiplicatively separable steady-state wave function [Eq. (76)] leads to sizeextensive molecular properties. In Sec. V A, we have defined the CI, CC-CI, and CC models [Eqs. (252)-(254)] in terms of the intermediate normalized wave function in Eq. (198), which in turn may be expressed in terms of the steadystate wave function using Eqs. (25) and (197). Therefore, to investigate the size-extensivity of molecular properties in the approximate models, CI, CC-CI, and CC, we will in the following examine the multiplicative separability of the intermediate normalized wave function for these models, both with respect to the parametrization of the unperturbed state and the time evolution of this state. For the CI, CC$\mathrm{CI}$, and CC models, we initially examine the multiplicative separability of the reference state $|\mathscr{R}\rangle$ and of the exponential operators, $\exp \left(B_{0}\right)$ and $\exp (B(t))$, entering the parametrization of the unperturbed state and the time evolution of this state. We will then examine the separability of the Jacobian matrix and molecular properties. 


\section{Separability of the reference and time-dependent states}

Let us initially assume that the reference state $|\mathscr{R}\rangle$ is multiplicatively separable,

$$
\left|\mathscr{R}^{C D}\right\rangle=\left|\mathscr{R}^{C}\right\rangle \otimes\left|\mathscr{R}^{D}\right\rangle,
$$

where $\otimes$ denotes that $\left|\mathscr{R}^{C D}\right\rangle$ is expressed in the direct product space referencing sub-system $C$ and $D$. We now consider the exponential operator $\exp (B)$. To obtain multiplicative separability,

$$
\exp \left(B^{C D}\right)=\exp \left(B^{C}+B^{D}\right)=\exp \left(B^{C}\right) \exp \left(B^{D}\right),
$$

the operator $B$ must be additively separable,

$$
B^{C D}=B^{C}+B^{D} .
$$

The last equality of Eq. (432) follows from Eq. (184).

When the operator $B$ is expressed in terms of the manybody excitation operators, $\tau_{\mu_{k}}$ of Eq. (250), it is additively separable for two non-interacting sub-systems $C$ and $D$,

$$
T^{C D}=T^{C}+T^{D},
$$

even for truncated excitation manifolds. This is wellestablished for the time-independent Hamiltonians, see, for example, Ref. 82, and the extension to the time-dependent Hamiltonians is straightforward. Eq. (434) is thus satisfied for both complete and truncated manifolds.

Turning our attention to the exponential operator expressed in terms of the state transfer operators, we find that the operator $S$ of Eq. (248) is not additively separable,

$$
S^{C D} \neq S^{C}+S^{D} .
$$

To see this, we express the exact operator $S$ for sub-systems $C$ and $D$ as

$$
\begin{aligned}
S^{C} & =\sum_{k} s_{k}\left|k^{C}\right\rangle\left\langle\mathscr{R}^{C}\right|, \\
S^{D} & =\sum_{k^{D}} s_{k} D\left|k^{D}\right\rangle\left\langle\mathscr{R}^{D}\right|,
\end{aligned}
$$

whereas for the compound system $C D$, the $S^{C D}$ operator in the direct product space becomes

$$
\begin{aligned}
S^{C D}= & \sum_{k^{C} k^{D}} s_{k} s_{k^{D}}\left(\left|k^{C}\right\rangle \otimes\left|k^{D}\right\rangle\right)\left(\left\langle\mathscr{R}^{C}\right| \otimes\left\langle\mathscr{R}^{D}\right|\right) \\
& +\sum_{k} s_{k} C\left(\left|k^{C}\right\rangle \otimes\left|\mathscr{R}^{D}\right\rangle\right)\left(\left\langle\mathscr{R}^{C}\right| \otimes\left\langle\mathscr{R}^{D}\right|\right) \\
& +\sum_{k^{D}} s_{k} D\left(\left|\mathscr{R}^{C}\right\rangle \otimes\left|k^{D}\right\rangle\right)\left(\left\langle\mathscr{R}^{C}\right| \otimes\left\langle\mathscr{R}^{D}\right|\right) .
\end{aligned}
$$

Using Eqs. (436) and (437), we may write Eq. (438) as

$$
S^{C D}=S^{C} \otimes S^{D}+S^{C} \otimes\left|\mathscr{R}^{D}\right\rangle\left\langle\mathscr{R}^{D}|+| \mathscr{R}^{C}\right\rangle\left\langle\mathscr{R}^{C}\right| \otimes S^{D},
$$

showing that the operator $S^{C D}$ is not additively separable, as expressed by Eq. (435). The exponential operator $\exp (S)$ is thus not multiplicatively separable, even for a complete excitation manifold.
We now examine how the lack of multiplicative separability of $\exp (S)$ influences the multiplicative separability of the intermediate normalized wave function $\exp (S)|\mathscr{R}\rangle$. To do that we use Eqs. (436)-(438) to write the intermediate normalized wave functions for sub-systems $C$ and $D$ and for the compound system $C D$ as

$$
\begin{gathered}
\exp \left(S^{C}\right)\left|\mathscr{R}^{C}\right\rangle=\left|\mathscr{R}^{C}\right\rangle+\sum_{k^{C}} s_{k}\left|k^{C}\right\rangle \\
\exp \left(S^{D}\right)\left|\mathscr{R}^{D}\right\rangle=\left|\mathscr{R}^{D}\right\rangle+\sum_{k^{D}} s_{k} D\left|k^{D}\right\rangle \\
\exp \left(S^{C D}\right)\left|\mathscr{R}^{C}\right\rangle \otimes\left|\mathscr{R}^{D}\right\rangle \\
=\left|\mathscr{R}^{C}\right\rangle \otimes\left|\mathscr{R}^{D}\right\rangle+\sum_{k} s_{k}\left|k^{C}\right\rangle \otimes\left|\Re^{D}\right\rangle \\
+\left|\mathscr{R}^{C}\right\rangle \otimes \sum_{k^{D}} s_{k} D\left|k^{D}\right\rangle+\sum_{k^{C} k^{D}} s_{k} C s_{k} D\left|k^{C}\right\rangle \otimes\left|k^{D}\right\rangle .
\end{gathered}
$$

For complete excitation manifolds, the compound wave function of Eq. (442) may be written as

$$
\begin{aligned}
& \exp \left(S^{C D}\right)\left|\mathscr{R}^{C}\right\rangle \otimes\left|\mathscr{R}^{D}\right\rangle \\
& =\left(\left|\mathscr{R}^{C}\right\rangle+\sum_{k} s_{k}\left|k^{C}\right\rangle\right) \otimes\left(\left|\mathscr{R}^{D}\right\rangle+\sum_{k^{D}} s_{k} D\left|k^{D}\right\rangle\right) \\
& \quad=\exp \left(S^{C}\right)\left|\mathscr{R}^{C}\right\rangle \otimes \exp \left(S^{D}\right)\left|\mathscr{R}^{D}\right\rangle, \quad \text { complete manifold, }
\end{aligned}
$$

where to obtain the last equality we have used Eqs. (440) and (441). For a complete manifold, the compound wave function $\exp \left(S^{C D}\right)\left|\mathscr{R}^{C}\right\rangle \otimes\left|\mathscr{R}^{D}\right\rangle$ is therefore multiplicatively separable, even though Eq. (432) is not satisfied. For truncated manifolds, however, the term $\sum_{k} C_{k} D s_{k} C s_{k} D\left|k^{C}\right\rangle \otimes$ $\left|k^{D}\right\rangle$ arising from the direct product of the sub-system wave functions of Eqs. (440) and (441) contains contributions that are beyond the excitation manifold of the compound system wave function of Eq. (442). The direct product and the compound wave functions therefore differ for truncated manifolds, even if the reference state is separable,

$$
\begin{aligned}
& \exp \left(S^{C D}\right)\left|\Re^{C}\right\rangle \otimes\left|\Re^{D}\right\rangle \\
& \quad \neq \exp \left(S^{C}\right)\left|\Re^{C}\right\rangle \otimes \exp \left(S^{D}\right)\left|\Re^{D}\right\rangle, \quad \text { truncated manifolds. }
\end{aligned}
$$

\section{Separability of the Jacobian}

We will now consider the Jacobian of the compound system CD and study under which conditions this matrix is separable and thereby gives excitation energies that are sizeintensive, i.e., independent of system size. Consider first the general form of the Jacobian given in Eq. (261), which for the 
compound system reads

$$
J_{k m}^{C D}=\left\langle k\left|\left[\left(H_{0}^{C}+H_{0}^{D}\right)^{B_{0}^{C D}}, \beta_{m}\right]\right| \mathscr{R}^{C D}\right\rangle .
$$

We will first show that the Jacobian of Eq. (445) is additively separable provided $B_{0}^{C D}$ and the excitation manifold $\{\beta\}$ used for the time-development are additively separable, and the reference state is multiplicatively separable,

$$
\begin{gathered}
B_{0}^{C D}=B_{0}^{C}+B_{0}^{D}, \\
\left|\mathscr{R}^{C D}\right\rangle=\left|\mathscr{R}^{C}\right\rangle \otimes\left|\mathscr{R}^{D}\right\rangle, \\
\left\{\beta^{C D}\right\}=\left\{\beta^{C}\right\} \oplus\left\{\beta^{D}\right\} .
\end{gathered}
$$

To show that the three conditions of Eq. (446) are sufficient to ensure separability of the Jacobian, we first note that the separability of the operator $B_{0}$ for the time-independent development ensures that the similarity-transformed Hamiltonian is extensive,

$$
\begin{aligned}
\left(H_{0}^{C}\right. & \left.+H_{0}^{D}\right)^{B_{0}^{C D}} \\
& =\exp \left(-\left(B_{0}^{C}+B_{0}^{D}\right)\right)\left(H_{0}^{C}+H_{0}^{D}\right) \exp \left(B_{0}^{C}+B_{0}^{D}\right) \\
& =\exp \left(-B_{0}^{C}\right) H^{C} \exp \left(B_{0}^{C}\right)+\exp \left(-B_{0}^{D}\right) H^{D} \exp \left(B_{0}^{D}\right) \\
& =\left(H_{0}^{C}\right)^{B_{0}^{C}}+\left(H_{0}^{D}\right)^{B_{0}^{D}} .
\end{aligned}
$$

Furthermore, the blocks of the Jacobian coupling the different sub-systems are then vanishing

$$
\begin{aligned}
J_{\mu_{k}^{C} v_{m}^{D}}^{C D} & =\left\langle\beta_{\mu_{k}^{C}} \mathscr{R}^{D}\left|\left[\left(H_{0}^{C}\right)^{B_{0}^{C}}+\left(H_{0}^{D}\right)^{B_{0}^{D}}, \beta_{v_{m}^{D}}\right]\right| \mathscr{R}^{C} \mathscr{R}^{D}\right\rangle \\
& =\left\langle\beta_{\mu_{k}^{C}} \mid \mathscr{R}^{C}\right\rangle\left\langle\mathscr{R}^{D}\left|\left[\left(H_{0}^{D}\right)^{B_{0}^{D}}, \beta_{v_{m}^{D}}\right]\right| \mathscr{R}^{D}\right\rangle=0,
\end{aligned}
$$

where we have used that $\left|\beta_{\mu_{k}^{C}}\right\rangle$ is orthogonal to $\left|\mathscr{R}^{C}\right\rangle$. For two operators referring to the same sub-system, the Jacobian reduces trivially to the standard sub-system Jacobian

$$
\begin{aligned}
J_{\mu_{k}^{C} v_{m}^{C}}^{C D} & =\left\langle\beta_{\mu_{k}^{C}} \mathscr{R}^{D}\left|\left[\left(H_{0}^{C}\right)^{B_{0}^{C}}, \beta_{v_{m}^{C}}\right]\right| \mathscr{R}^{C} \mathscr{R}^{D}\right\rangle \\
& =\left\langle\beta_{\mu_{k}^{C}}\left|\left[\left(H_{0}^{C}\right)^{B_{0}^{C}}, \beta_{v_{m}^{C}}\right]\right| \mathscr{R}^{C}\right\rangle=J_{\mu_{k}^{C} v_{m}^{C}}^{C} .
\end{aligned}
$$

From Eqs. (448) and (449), we therefore have the additive separability of the Jacobian,

$$
J^{C D}=J^{C} \oplus J^{D} .
$$

Any model, exact or approximate, fulfilling the conditions of Eq. (446) will therefore provide an additively separable Jacobian.

It is important to note that there are size-extensive methods that do not fulfill Eq. (446) but still provide sizeintensive excitation energies. Consider, for example, the models, including the full CC-CI and CI models, where the excitation manifold for the time-development have the form of Eq. (439), rather than that of Eq. (446c). For this form of the excitation manifold, the blocks of the Jacobian coupling any two of the three types of excitations vanish and the blocks containing excitations only in one of the systems reduce as Eq. (449). For two excitations containing excitations in both sub-systems, one obtains for the CI-model

$$
\begin{aligned}
& J_{\mu_{k}^{C}}^{C D} \mu_{k}^{D}, v_{m}^{C} v_{m}^{D} \\
& =\left\langle\mu_{k}^{C} \mu_{k}^{D}\left|\left[H_{0}^{C}+H_{0}^{D},\left|v_{m}^{C}\right\rangle\left\langle 0_{0}^{C}|\otimes| v_{m}^{D}\right\rangle\left\langle 0_{0}^{D}\right|\right]\right| 0_{0}^{C} 0_{0}^{D}\right\rangle \\
& =\left\langle\mu_{k}^{C}\left|H_{0}^{C}\right| v_{m}^{C}\right\rangle \delta_{\mu_{k}^{D} v_{m}^{D}}+\delta_{\mu_{k}^{C} v_{m}^{C}}\left\langle\mu_{k}^{D}\left|H_{0}^{D}\right| v_{m}^{D}\right\rangle \\
& -\delta_{\mu_{k}^{C} v_{m}^{C}} \delta_{\mu_{k}^{D} v_{m}^{D}}\left(E_{0}^{C}+E_{0}^{D}\right) \\
& =J_{\mu_{k}^{C} v_{m}^{C}}^{C} \delta_{\mu_{k}^{D} v_{m}^{D}}+\delta_{\mu_{k}^{C} v_{m}^{C}} J_{\mu_{k}^{D} v_{m}^{D}}^{D} .
\end{aligned}
$$

Using a full linear expansion for the time-development, one obtains therefore the following separation of the Jacobian:

$$
J^{C D}=J^{C} \oplus J^{D} \oplus\left(J^{C} \otimes 1^{D}+1^{C} \otimes J^{D}\right) .
$$

\begin{tabular}{|c|c|c|c|}
\hline & $\mathrm{CC}$ & $\mathrm{CI}$ & $\mathrm{CC}-\mathrm{CI}$ \\
\hline Manifold, TI & $\left\{\tau^{C}\right\} \oplus\left\{\tau^{D}\right\}$ & $S^{C} \otimes\left|\mathscr{R}^{D}\right\rangle\left\langle\mathscr{R}^{D}|\oplus| \mathscr{R}^{C}\right\rangle\left\langle\mathscr{R}^{C}\right| \otimes S^{D} \oplus S^{C} \otimes S^{D}$ & $\left\{\tau^{C}\right\} \oplus\left\{\tau^{D}\right\}$ \\
\hline Manifold, TD & $\left\{\tau^{C}\right\} \oplus\left\{\tau^{D}\right\}$ & $S^{C} \otimes\left|\mathscr{R}^{D}\right\rangle\left\langle\mathscr{R}^{D}|\oplus| \mathscr{R}^{C}\right\rangle\left\langle\mathscr{R}^{C}\right| \otimes S^{D} \oplus S^{C} \otimes S^{D}$ & $S^{C} \otimes\left|\mathscr{R}^{D}\right\rangle\left\langle\mathscr{R}^{D}|\oplus| \mathscr{R}^{C}\right\rangle\left\langle\mathscr{R}^{C}\right| \otimes S^{D} \oplus S^{C} \otimes S^{D}$ \\
\hline Jacobian, full expansion & $J^{C} \oplus J^{D}$ & $J^{C} \oplus J^{D} \oplus\left(J^{C} \otimes 1^{D}+1^{C} \otimes J^{D}\right)$ & $J^{C} \oplus J^{D} \oplus\left(J^{C} \otimes 1^{D}+1^{C} \otimes J^{D}\right)$ \\
\hline $\begin{array}{l}\text { Jacobian, truncated } \\
\text { expansion }\end{array}$ & $J^{C} \oplus J^{D}$ & Not separable & $J^{C} \oplus J^{D} \oplus$ non-separable part \\
\hline Excitation energies & Size-intensive & Not size-intensive & Size-intensive \\
\hline Molecular properties & Size-extensive & Not size-extensive & Not size-extensive \\
\hline
\end{tabular}

The eigenvalues of the first two parts of the Jacobian are the excitation energies of the individual systems, whereas the eigenvalues of the last term are the sum of excitation energies of the two sub-systems. As the excitation energies of the individual systems are correctly reproduced, these models yield size-intensive excitation energies.

Armed with the above general developments, we are now able to discuss the separability of the Jacobian for the three wave function models, CC, CI, and CC-CI. The results are summarized in Table I, where we also summarize our later conclusions concerning the separability of molecular properties.

For the CC model, where both manifolds are additively separable, the Jacobian is also additively separable, independent of whether a complete or truncated expansion is used. The excitation energies obtained as eigenvalues of the compound Jacobian become the excitation energies of $\mathrm{C}$ and $\mathrm{D}$, and the $\mathrm{CC}$ model therefore yields size-intensive

TABLE I. Summary of separability of excitation manifolds, Jacobians, excitation energies, and molecular properties for the standard wave function models. 
excitation energies. For the CI-model, we obtain a Jacobian of the form of Eq. (452) when no truncations are performed. The eigenvalues of the first two parts of the Jacobian are the excitation energies of the individual systems, whereas the eigenvalues of the last term are the sum of excitation energies of the two sub-systems. For truncated CI-expansions, where the manifolds for both the time-independent and timedependent parts are truncated, the reference state and thereby the Jacobian are not separable.

Consider finally the CC-CI model, where different excitation manifolds are used for the time-independent and the time-dependent parts. Without truncations, the CC-CI Jacobian behaves as the CI Jacobian and gives the individual plus the combined excitation energies. However, when the excitation manifold is truncated, a difference between the $\mathrm{CI}$ and CC-CI models occurs. As the first two conditions of Eq. (446) are fulfilled for the CC-CI model, the Jacobian becomes a direct sum of the sub-system Jacobians and a part containing excitations in both systems. The parts of the Jacobian referring to the individual sub-systems are independent of whether one considers this sub-system or the compound system, so this model correctly reproduces the excitations of the individual systems. The part of the Jacobian containing the combined excitations in the two subsystems does not separate into direct products of the subsystem Jacobians and gives therefore a non-separable block of the Jacobian, resulting in excitation energies that are not size-intensive and less accurate than those obtained from the sub-system Jacobians.

\section{Size-extensivity of molecular properties}

Applying the above analysis to the $\mathrm{CI}, \mathrm{CC}-\mathrm{CI}$, and $\mathrm{CC}$ models, we find that for the CI model [Eq. (252)] it follows from Eq. (444) that already the unperturbed state is not multiplicatively separable when truncations are introduced in the excitation manifold and the truncated CI model therefore cannot yield size-extensive molecular properties.

In the CC-CI and CC models, the CC state is used as the unperturbed state and this state is multiplicatively separable, as follows from Eq. (434). The time evolution in the CCCI model is, however, linearly parametrized in terms of the $\exp (S(t))$ operator, and therefore, as follows from Eq. (444), the time-dependent wave function is not multiplicatively separable for this model, when truncations are introduced in the excitation manifold. Consequently, molecular response properties are not size-extensive in truncated CC-CI models. In the $\mathrm{CC}$ model, the time evolution is parametrized in terms of the $\exp (T(t))$ operator and, as follows from Eq. (434), the time-dependent wave function is multiplicatively separable, provided that the reference state is multiplicatively separable. Molecular response properties are, therefore, size-extensive in the $\mathrm{CC}$ model, even if truncations are introduced in the excitation manifold. For the CC-CI model, the non-sizeextensivity is weak because, compared to the CC model, we are neglecting only non-linear terms for the time-evolving state and these non-linear terms only enter when excitations are simultaneously considered for the two sub-systems in the compound system.
Let us finally consider size-extensivity of molecular properties obtained as residues of response functions. When a perturbation frequency matches an excitation energy of the unperturbed system, the frequency-dependent quasi-energy correction of Eq. (65) becomes infinite and a number of molecular properties are determined as residues of the response function, for example [Eq. (71)],

$$
\begin{aligned}
& \lim _{\omega_{X_{2} \rightarrow \omega}}\left(\omega_{X_{2}}-\omega\right) \mathcal{E}^{X_{j_{1}} X_{j_{2}}\left(\omega_{X_{j_{1}}}, \omega_{X_{j_{2}}}\right)} \\
& =\sum_{m: E_{m}-E_{0}=\omega}\left\langle 0_{0}\left|X_{j_{1}}\right| 0_{m}\right\rangle\left\langle 0_{m}\left|X_{j_{2}}\right| 0_{0}\right\rangle .
\end{aligned}
$$

For an exact wave function, the quasi-energy corrections are size-extensive [Eq. (84)] and excitation energies are sizeintensive and therefore it follows from Eq. (453) that the residues are also size-extensive. For truncated CI models, transition properties are not size-extensive, because neither excitation energies are size intensive nor quasi-energy corrections are size-extensive. For the CC-CI model, excitation energies are size intensive, but the quasi-energy corrections are not size-extensive for truncated manifolds, and therefore, the CC-CI transition properties are not size-extensive for the truncated manifolds. In the $\mathrm{CC}$ model, even when truncations are introduced in the excitation manifold, excitation energies are size-intensive and quasi-energy corrections are size-extensive, and therefore, transition properties are size-extensive.

\section{Linear versus exponential parametrization in the $\mathrm{Cl}, \mathrm{CC}-\mathrm{Cl}$ and $\mathrm{CC}$ models}

We now compare the CI, CC-CI, and CC molecular response properties in the context of linear versus exponential parametrization of the unperturbed state and its time evolution. For the CI, CC-CI, and CC models, different parametrizations are used for the wave function and the response functions are structurally very different [see, for example, the linear response functions in Eqs. (299), (340), and (383)]. However, in the limit where no truncation is performed in the excitation manifold (the FCI limit), the results obtained with the three models are identical, as in this limit, the Schrödinger equation for all three models is solved with no approximations, just in terms of different parametrizations. However, when truncations are performed in the excitation manifold, different results are obtained for the three models.

For the CI model, the unperturbed state satisfies the variational condition. This gives large simplifications in the response functions. In particular, the ${ }^{c} \mathcal{L}_{H_{0}}^{N L}$ term in Eq. (270a) vanishes in the $\mathrm{CI}$ model. The linear response function for the CI model in Eq. (299) therefore only contains one single term. For both the CC-CI and CC models, a coupled cluster unperturbed state is used. The coupled cluster unperturbed state satisfies the cluster amplitudes and multipliers equations [Eqs. (325) and (327)]. These equations also introduce simplifications in the response functions, but to less extent than the variational condition. As a result, the CC-CI [Eq. (340)] and CC [Eq. (383)] linear response functions contain additional terms compared to the CI linear response function [Eq. (299)]. 
Comparing the expressions for the CC-CI and CC response functions, we see that the non-linear terms containing the $H_{0}^{T_{0}}$ operator are greatly simplified for the CC-CI model compared to the $\mathrm{CC}$ model. The CC-CI response functions thus only contain one non-linear $H_{0}^{T_{0}}$ term [Eq. (270a)], whereas the $\mathrm{CC}$ response functions have, in general, four non-linear $H_{0}^{T_{0}}$ terms [Eq. (270b)]. Comparing the CC-CI linear response function [Eq. (340)] with the CC linear response function [Eq. (383)], we therefore also see that the CC linear response functions contain an additional term (the first term in Eq. (383)) compared to the CC-CI linear response function and also that the second term in Eq. (383) has a more involved structural form than its counterpart in the first term of Eq. (340). For the quadratic and higher-order response functions, this difference between the number and the complexity of the terms entering the CC and CC-CI response functions becomes even more pronounced, resulting in much simpler expressions for the $\mathrm{CC}-\mathrm{CI}$ than for the $\mathrm{CC}$ response functions.

For the CI and CC-CI models, the time evolution is described in terms of a linear parametrization and an explicit representation is therefore obtained for the ground and excited states. However, this is obtained at the expense that these models do not yield molecular properties that are size-extensive. This may, for example, be seen explicitly in the CC-CI linear response function that contains a term (the first term in Eq. (340)) that increases quadratically with system size. Contrary, for the CC model, where the time evolution is exponentially parametrized, molecular properties are size-extensive, but we cannot obtain an explicit representation of the ground and excited states. However, this is not important as we have a procedure for determining molecular properties without recourse to an explicit representation of individual states.

\section{Comparison of $\mathrm{CC}-\mathrm{Cl}$ and EOMCC molecular response properties}

We now compare the CC-CI and EOMCC molecular response properties. For first-order molecular properties, we obtain from Eqs. (335) and (388)

$$
\left\langle\left\langle X_{j_{1}}\right\rangle\right\rangle_{0}^{\mathrm{CC}-\mathrm{CI}}=\left\langle\left\langle X_{j_{1}}\right\rangle\right\rangle_{0}^{\mathrm{EOMCC}} .
$$

In the supplementary material, ${ }^{89}$ we have shown explicitly that the CC-CI and EOMCC linear response functions are equal,

$$
\left\langle\left\langle X_{j_{1}} ; X_{j_{2}}\right\rangle\right\rangle_{\omega_{X_{j_{2}}}}^{\mathrm{CC}-\mathrm{CI}}=\left\langle\left\langle X_{j_{1}} ; X_{j_{2}}\right\rangle\right\rangle_{\omega_{X_{j_{2}}}}^{\mathrm{EOMCC}},
$$

and that the $\mathrm{CC}-\mathrm{CI}$ and EOMCC quadratic response functions differ,

$$
\left\langle\left\langle X_{j_{1}} ; X_{j_{2}}, X_{j_{3}}\right\rangle\right\rangle_{\omega_{X_{j_{2}}}, \omega_{X_{j_{3}}}}^{\mathrm{CC}-\mathrm{CI}} \neq\left\langle\left\langle X_{j_{1}} ; X_{j_{2}}, X_{j_{3}}\right\rangle\right\rangle_{\omega_{X_{j_{2}}}, \omega_{X_{j_{3}}}}^{\mathrm{EOMCC}} .
$$

We now show that this difference holds also for cubic and higher-order response functions and that only the CC-CI model reproduces FCI results.

The EOMCC model is defined by the non-Hermitian eigenvalue equation [Eqs. (346)-(349)]. The optimization condition for the EOMCC ground state is defined by vanishing Hamiltonian matrix elements between the ground state and the EOMCC excited states,

$$
\left\langle 0_{n}^{\mathrm{CC}}\left|H_{0}\right| 0_{0}^{\mathrm{CC}}\right\rangle=0, \quad n>0,
$$

$$
\left\langle 0_{0}^{\mathrm{CC}}\left|H_{0}\right| 0_{n}^{\mathrm{CC}}\right\rangle=0, \quad n>0 .
$$

Further, the excited states in the EOMCC model are obtained from a diagonalization of the similarity transformed Hamiltonian in Eq. (350) and the excited states are therefore linearly expanded in the $\left|\mathcal{B}^{T_{0}}\right\rangle$ [Eq. (332)] and $\left\langle\mathcal{B}^{T_{0}}\right|$ [Eq. (345)] basis.

We now consider the optimization conditions in more detail. Substituting Eqs. (366) and (369) in Eq. (457), we obtain

$$
\left\langle 0_{n}^{\mathrm{CC}}\left|H_{0}\right| 0_{0}^{\mathrm{CC}}\right\rangle=\sum_{\mu_{k}}{ }^{\mathrm{CC}} \bar{C}_{n \mu_{k}}\left\langle\mu_{k}^{\mathrm{CC}}\left|H_{0}^{T_{0}}\right| \mathrm{HF}\right\rangle=0 .
$$

The optimization condition in Eq. (457) is thus equivalent to requiring that the cluster amplitudes equation [Eq. (325)] is satisfied for the unperturbed system. Inserting Eqs. (367) and (368) in Eq. (458) gives

$$
\begin{aligned}
\left\langle 0_{0}^{\mathrm{CC}}\left|H_{0}\right| 0_{n}^{\mathrm{CC}}\right\rangle= & \sum_{\mu_{k}}{ }^{\mathrm{CC}} C_{\mu_{k} n}\left[\left\langle\mathrm{HF}\left|H_{0}^{T_{0}}\right| \mu_{k}^{\mathrm{CC}}\right\rangle\right. \\
& +\sum_{v_{m}} \bar{t}_{v_{m}}^{(0)}\left(\left\langle v_{m}^{\mathrm{CC}}\left|H_{0}^{T_{0}}\right| \mu_{k}^{\mathrm{CC}}\right\rangle\right. \\
& \left.\left.-\left\langle\mathrm{HF}\left|H_{0}^{T_{0}}\right| \mathrm{HF}\right\rangle \delta_{v_{m} \mu_{k}}-\bar{t}_{\mu_{k}}^{(0)}\left\langle v_{m}^{\mathrm{CC}}\left|H_{0}^{T_{0}}\right| \mathrm{HF}\right\rangle\right)\right] \\
= & 0 .
\end{aligned}
$$

The CC amplitudes equation [Eq. (325)] is satisfied and the last term in Eq. (460) therefore vanishes, giving

$$
\sum_{\mu_{k}}{ }^{\mathrm{CC}} C_{\mu_{k} n}\left[\eta_{\mu_{k}}+\sum_{v_{m}} \bar{t}_{v_{m}}^{(0)}{ }^{\mathrm{CC}} J_{v_{m} \mu_{k}}\right]=0
$$

where we have used Eqs. (328) and (329). The optimization condition in Eq. (458) is thus equivalent to requiring that the CC multipliers satisfy the multipliers equation [Eq. (327)] for the unperturbed system. The excited states in the EOMCC model are further linearly expanded in the $\left|\mathcal{B}^{T_{0}}\right\rangle$ and $\left\langle\mathcal{B}^{T_{0}}\right|$ basis. Such linear expansions can only be obtained when the time evolution is described in terms of a linear expansion. EOMCC response functions therefore should be defined by a model where the CC amplitude and multiplier equations are satisfied for the unperturbed system and where the time evolution is described in terms of a linear expansion. These are precisely the premises for the CC-CI model [Eq. (254)], in accordance with the fact that static CC-CI molecular properties are obtained from finite field EOMCC energy calculations, as discussed in Sec. X A 3.

In Sec. IX, we have discussed how the EOMCC response functions are obtained expressing the CI response functions of Sec. VI in the basis of the eigenstates and energies $\left\{\left|0_{n}^{\mathrm{CI}}\right\rangle,\left\langle 0_{n}^{\mathrm{CI}}\right|,{ }^{\mathrm{CI}} E_{n}\right\}, n=0,1,2, \ldots$ of the $\mathrm{CI}$ eigenvalue equation in Eq. (304) and replacing the CI eigenstates and energies with the eigenstates and energies $\left\{\left|0_{n}^{\mathrm{CC}}\right\rangle,\left\langle 0_{n}^{\mathrm{CC}}\right|,{ }^{\mathrm{CC}} E_{n}\right\}, n$ $=0,1,2, \ldots$ of the EOMCC eigenvalue equation in Eqs. (346) and (347). However, when the CI response functions are determined, it is done using a stronger optimization condition than is satisfied for the EOMCC model. For the EOMCC model, the $\mathrm{CC}$ amplitude and multiplier equations are 
satisfied for the unperturbed system. In CI theory, the amplitude and multiplier equations are also satisfied, but in addition we have

$$
\left\langle 0_{n}^{\mathrm{CI}}\left|H_{0}\right| 0_{0}^{\mathrm{CI}}\right\rangle^{\dagger}=\left\langle 0_{0}^{\mathrm{CI}}\left|H_{0}\right| 0_{n}^{\mathrm{CI}}\right\rangle=0,
$$

which is not satisfied for EOMCC, because $H_{0}^{T_{0}}$ is not Hermitian. The stronger optimization condition [Eq. (462)] implies that the ${ }^{c} \mathcal{L}_{H_{0}}^{N L}$ term [Eq. (270a)],

$$
\begin{aligned}
{ }^{c} \mathcal{L}_{H_{0}}^{N L}= & \sum_{K L M} \sum_{k} \bar{s}_{k}\left(\omega_{K}\right) s_{k}\left(\omega_{L}\right) \sum_{m} s_{m}\left(\omega_{M}\right) \\
& \times\left\langle 0_{0}^{\mathrm{CI}}\left|H_{0}\right| 0_{m}^{\mathrm{CI}}\right\rangle \Delta\left(\omega_{K}+\omega_{L}+\omega_{M}\right),
\end{aligned}
$$

vanishes in CI. This term is therefore not present in the EOMCC response functions in Sec. IX. The CC-CI and EOMCC response functions therefore differ when this term contributes to the CC-CI response functions. When this term is neglected, FCI results cannot be obtained using the EOMCC response functions of Sec. IX. Contrary, the CC-CI model gives FCI limit results.

The ${ }^{c} \mathcal{L}_{H_{0}}^{N L}$ term [Eq. (463)] does not contribute when first-order amplitude equations are determined [see Eq. (338)] and EOMCC and CC-CI first-order amplitudes are therefore identical. When the linear response function is calculated using the $2 n+1$ and $2 n+2$ rules, only the first-order amplitudes are used and the EOMCC and CC-CI linear response functions therefore become identical. However, if the linear response function is determined in the asymmetric form, where first-order multipliers are used to determine the linear response function, the EOMCC linear response function is not equal to the one obtained when the $2 n+1$ and $2 n+2$ rules are applied and is not able to reproduce FCI result. EOMCC response functions in general are not capable of reproducing FCI results.

\section{SUMMARY, CONCLUSION, AND OUTLOOK}

For a time-periodic perturbation, we have recasted the time-dependent Schrödinger equation into a Hermitian eigenvalue equation by carrying out time averaging over one period of the perturbation in the composite Hilbert space. The solution to the time-dependent Schrödinger equation for a time-periodic perturbation thereby becomes a straightforward generalization of the solution to the Hermitian eigenvalue equation for a static perturbation. The Hermitian eigenvalue equation may be solved using the same techniques as for the static perturbation. The Hermitian eigenvalue equation has the quasi-energy as an eigenvalue and the time-periodic regular wave function as the corresponding eigenstate. Differentiation of the quasi-energy with respect to the perturbation strengths determines molecular response functions. We have thus arrived at a rigorous and transparent formulation of response function theory applicable to both variational and non-variational wave functions where both a linear and a non-linear parametrization of the time evolution can be used and where the optimization of the wave function for the unperturbed and perturbed system may be carried out differently.

We have illustrated the generality and simplicity of the new formulation of response function theory by deriving response functions for a $\mathrm{CI}$ model, where both the unperturbed state and its time evolution are linearly parametrized, and for a coupled cluster unperturbed state, where we have considered both a linear (CC-CI model) and a non-linear (CC model) parametrization of its time evolution. We have shown that the CC-CI molecular properties in the timeindependent limit become an analytic representation of the static properties obtained from EOMCC finite field energy calculations. We have carried out a detailed comparison of traits of response functions obtained for $\mathrm{CI}, \mathrm{CC}$, and $\mathrm{CC}-\mathrm{CI}$ models with emphasis on linear versus non-linear parametrizations of the time evolution. The linear parametrization of the time-evolution in the $\mathrm{CI}$ and $\mathrm{CC}-\mathrm{CI}$ models allows for an explicit representation of the ground and excited states, but at the expense of having molecular properties that are not size-extensive. For an exponential parametrization of the time-evolution of the $\mathrm{CC}$ state, an explicit representation of the ground and excited states cannot be obtained. However, this is not important as explicit expressions can be obtained for molecular properties that in turn are sizeextensive. A detailed analysis has further shown that the non-size-extensivity of CC-CI molecular properties is weak. Taking into consideration the simplifications that arise when molecular properties are calculated using the CC-CI rather than the CC model, the CC-CI model may provide an alternative and practical vehicle for determining molecular properties.

The development we have presented has thus brought the definition of molecular response properties and their determination on par for static and time-periodic perturbations, and it has removed inadequacies and inconsistencies of previous response function theory formulations. The development has also allowed response functions to be determined for new wave function models, e.g., the CC-CI model. In a later publication, we will also show how the Hermitian eigenvalue equation may be solved using perturbation theory and how response functions may then be determined for, for example, $\Lambda \operatorname{CCSD}[\mathrm{T}]^{90-92}$ and $\operatorname{CCSD}(\mathrm{T}-2)^{93}$ reference states.

\section{ACKNOWLEDGMENTS}

F.P. and P.J. acknowledge support from The European Research Council under the European Union's (EU) Seventh Framework Programme (FP/2007-2013)/ERC Grant Agreement No. 291371 and P.J. and J.O. acknowledge support from The Danish Council for Independent Research-Natural Sciences. F.P. also acknowledges support form the Polish National Science Centre (Project No. 3714/B/H03/2011/40).

${ }^{1}$ J. Olsen and P. Jørgensen, J. Chem. Phys. 82, 3235 (1985).

${ }^{2}$ O. Christiansen, P. Jørgensen, and C. Hättig, Int. J. Quantum Chem. 68, 1 (1998).

${ }^{3}$ G. Floquet, Ann. Ecole Norm. Sup. 2 12, 47 (1883), available online at http://www.numdam.org/item?id=ASENS_1883_2_12_47_0.

${ }^{4}$ D. J. Tannor, Introduction to Quantum Mechanics: A Time-dependent Perspective (University Science Books, 2007). 
${ }^{5}$ S. Blanes, F. Casas, J. A. Oteo, and J. Ros, Phys. Rep. 470, 151 (2009). ${ }^{6}$ H. Sambe, Phys. Rev. A 7, 2203 (1973).

${ }^{7}$ D. N. Zubarev, Nonlinear Statistical Thermodynamics (Consultant Bureau, Plenum, New York, 1974).

${ }^{8} \mathrm{~J}$. Linderberg and Y. Öhrn, Propagators in Quantum Chemistry (Academic Press, New York, 1973).

${ }^{9}$ A. D. McLachlan and M. A. Ball, Rev. Mod. Phys. 36, 844 (1964).

${ }^{10}$ P. Jørgensen, Annu. Rev. Phys. Chem. 26, 359 (1975).

${ }^{11}$ J. Oddershede, P. Jørgensen, and D. L. Yeager, Comput. Phys. Rep. 2, 33 (1984).

${ }^{12}$ J. Olsen and P. Jørgensen, in Modern Electronic Structure Theory II, edited by D. Yarkoni (VCH, New York, 1995).

${ }^{13}$ P. Jørgensen, H. J. A. Jensen, and J. Olsen, J. Chem. Phys. 89, 3654 (1988).

${ }^{14}$ H. Hettema, H. J. A. Jensen, P. Jørgensen, and J. Olsen, J. Chem. Phys. 97, 1174 (1992).

${ }^{15} \mathrm{O}$. Vahtras, H. Ågren, P. Jørgensen, H. J. A. Jensen, T. Helgaker, and J. Olsen, J. Chem. Phys. 97, 9178 (1992).

${ }^{16}$ P. Norman, D. Jonsson, O. Vahtras, and H. Ågren, Chem. Phys. Lett. 242, 7 (1995).

${ }^{17}$ D. Jonsson, P. Norman, and H. Ågren, J. Chem. Phys. 105, 6401 (1996).

${ }^{18}$ M. Jaszuński and A. Rizzo, Mol. Phys. 96, 855 (1999).

${ }^{19}$ V. Carravetta, H. Ågren, H. J. A. Jensen, P. Jørgensen, and J. Olsen, J. Phys. B: At., Mol. Opt. Phys. 22, 2133 (1989).

${ }^{20}$ V. Spirko, H. J. A. Jensen, and P. Jørgensen, Chem. Phys. 144, 343 (1990).

${ }^{21}$ J. M. Schins, L. D. A. Siebbeles, J. Los, M. Kristensen, and H. Koch, J. Chem. Phys. 93, 3887 (1990).

${ }^{22}$ D. Nordfors, H. Ågren, and H. J. A. Jensen, Int. J. Quantum Chem. 40, 475 (1991).

${ }^{23}$ H. J. A. Jensen, P. Jørgensen, H. Hettema, and J. Olsen, Chem. Phys. Lett. 187, 387 (1991).

${ }^{24}$ M. Jaszuński, P. Jørgensen, H. Koch, H. Ågren, and T. Helgaker, J. Chem. Phys. 98, 7229 (1993).

${ }^{25} \mathrm{H}$. Ågren, V. Carravetta, H. J. A. Jensen, P. Jørgensen, and J. Olsen, Phys. Rev. A 47, 3810 (1993).

${ }^{26}$ B. Minaev, O. Vahtras, and H. Ågren, Chem. Phys. 208, 299 (1996).

${ }^{27}$ B. F. Minaev, N. A. Murugan, and H. Ågren, Int. J. Quantum Chem. 113, 1847 (2013).

${ }^{28}$ P. Norman, Y. Luo, D. Jonsson, and H. Ågren, J. Chem. Phys. 106, 1827 (1997).

${ }^{29}$ P. Norman, D. Jonsson, and H. Ågren, Chem. Phys. Lett. 268, 337 (1997).

${ }^{30}$ D. Jonsson, P. Norman, O. Vahtras, H. Ågren, and A. Rizzo, J. Chem. Phys. 106, 8552 (1997).

${ }^{31}$ D. Jonsson and P. Norman, J. Chem. Phys. 109, 572 (1998).

${ }^{32}$ H. Koch and P. Jørgensen, J. Chem. Phys. 93, 3333 (1990).

${ }^{33}$ H. Koch, H. J. A. Jensen, P. Jørgensen, and T. Helgaker, J. Chem. Phys. 93, 3345 (1990).

${ }^{34}$ H. Koch, R. Kobayashi, A. Sanchez de Meras, and P. Jørgensen, J. Chem. Phys. 100, 4393 (1994).

${ }^{35}$ H. Koch, O. Christiansen, R. Kobayashi, P. Jørgensen, and T. Helgaker, Chem. Phys. Lett. 228, 233 (1994).

${ }^{36}$ F. Aiga, K. Sasagane, and R. Itoh, J. Chem. Phys. 99, 3779 (1993).

${ }^{37}$ K. Sasagane, F. Aiga, and R. Itoh, J. Chem. Phys. 99, 3738 (1993).

${ }^{38}$ E. A. Hylleraas, Z. Phys. 65, 209 (1930).

${ }^{39}$ E. Wigner, Math. Natur. Anz. (Budapest) 53, 477 (1935).

${ }^{40}$ T. Helgaker and P. Jørgensen, in Methods in Computational Molecular Physics, edited by S. Wilson and G. H. F. Diercksen (Plenum Press, New York, 1992), Vol. 293, pp. 353-421.

${ }^{41}$ T. Helgaker and P. Jørgensen, Theor. Chim. Acta 75, 111 (1989).

${ }^{42}$ O. Christiansen, H. Koch, and P. Jørgensen, Chem. Phys. Lett. 243, 409 (1995).

${ }^{43}$ G. D. Purvis and R. J. Bartlett, J. Chem. Phys. 76, 1910 (1982).

${ }^{44}$ H. Koch, O. Christiansen, P. Jørgensen, A. M. Sanchez de Merás, and T. Helgaker, J. Chem. Phys. 106, 1808 (1997).

${ }^{45}$ O. Christiansen, H. Koch, and P. Jørgensen, J. Chem. Phys. 103, 7429 (1995).

${ }^{46}$ C. E. Smith, R. A. King, and T. D. Crawford, J. Chem. Phys. 122, 054110 (2005).

${ }^{47}$ J. Noga and R. J. Bartlett, J. Chem. Phys. 86, 7041 (1987); erratum, 89, 3401 (1988).

${ }^{48}$ G. E. Scuseria and H. F. Schaefer, Chem. Phys. Lett. 152, 382 (1988)

${ }^{49}$ G. Trucks, J. Noga, and R. Bartlett, Chem. Phys. Lett. 145, 548 (1988).

${ }^{50}$ J. Noga, R. Bartlett, and M. Urban, Chem. Phys. Lett. 134, 126 (1987).

${ }^{51}$ M. Urban, J. Noga, S. Cole, and R. Bartlett, J. Chem. Phys. 83, 4041 (1985); erratum, 85, 5383 (1986).

${ }^{52}$ Y. Lee, S. Kucharski, and R. Bartlett, J. Chem. Phys. 81, 5906 (1984); erratum, 82, 5761 (1985).
${ }^{53}$ Y. Lee and R. Bartlett, J. Chem. Phys. 80, 4371 (1984).

${ }^{54}$ D. H. Magers, R. J. Harrison, and R. J. Bartlett, J. Chem. Phys. 84, 3284 (1986).

${ }^{55}$ O. Christiansen, H. Koch, A. Halkier, P. Jørgensen, T. Helgaker, and A. Sanchez de Meras, J. Chem. Phys. 105, 6921 (1996).

${ }^{56} \mathrm{O}$. Christiansen, A. Halkier, H. Koch, P. Jørgensen, and T. Helgaker, J. Chem. Phys. 108, 2801 (1998).

${ }^{57}$ F. Pawłowski, P. Jørgensen, and C. Hättig, Chem. Phys. Lett. 389, 413 (2004).

${ }^{58}$ R. Kobayashi, H. Koch, and P. Jørgensen, Chem. Phys. Lett. 219, 30 (1994),

${ }^{59}$ O. Christiansen, J. Gauss, and J. F. Stanton, Chem. Phys. Lett. 292, 437 (1998).

${ }^{60}$ K. Hald, F. Pawłowski, P. Jørgensen, and C. Hättig, J. Chem. Phys. 118, 1292 (2003).

${ }^{61}$ C. Hättig, O. Christiansen, H. Koch, and P. Jørgensen, Chem. Phys. Lett. 269, 428 (1997).

${ }^{62}$ C. Hättig, O. Christiansen, and P. Jørgensen, Chem. Phys. Lett. 282, 139 (1998).

${ }^{63}$ J. Gauss, O. Christiansen, and J. F. Stanton, Chem. Phys. Lett. 296, 117 (1998).

${ }^{64}$ F. Pawłowski, P. Jørgensen, and C. Hättig, Chem. Phys. Lett. 391, 27 (2004).

${ }^{65}$ F. Pawłowski, P. Jørgensen, and C. Hättig, Chem. Phys. Lett. 413, 272 (2005).

${ }^{66}$ M. Pecul, F. Pawłowski, P. Jørgensen, A. Köhn, and C. Hättig, J. Chem. Phys. 124, 114101 (2006).

${ }^{67}$ A. J. Thorvaldsen, K. Ruud, K. Kristensen, P. Jørgensen, and S. Coriani, J. Chem. Phys. 129, 214108 (2008).

${ }^{68}$ T. Kjærgaard, P. Jørgensen, A. J. Thorvaldsen, P. Sałek, and S. Coriani, J. Chem. Theory Comput. 5, 1997 (2009).

${ }^{69}$ A. J. Thorvaldsen, L. Ferrighi, K. Ruud, H. Ågren, S. Coriani, and P. Jørgensen, Phys. Chem. Chem. Phys. 11, 2293 (2009).

${ }^{70}$ P. Norman, D. M. Bishop, H. J. A. Jensen, and J. Oddershede, J. Chem. Phys. 115, 10323 (2001).

${ }^{71}$ P. Norman, D. M. Bishop, H. J. A. Jensen, and J. Oddershede, J. Chem. Phys. 123, 194103 (2005).

${ }^{72}$ L. Jensen, J. Autschbach, and G. C. Schatz, J. Chem. Phys. 122, 224115 (2005).

${ }^{73}$ D. Rocca, R. Gebauer, Y. Saad, and S. Baroni, J. Chem. Phys. 128, 154105 (2008).

${ }^{74}$ S. Coriani, T. Fransson, O. Christiansen, and P. Norman, J. Chem. Theory Comput. 8, 1616 (2012).

${ }^{75} \mathrm{~K}$. Kristensen, J. Kauczor, T. Kjærgaard, and P. Jørgensen, J. Chem. Phys. 131, 044112 (2009).

${ }^{76}$ T. Helgaker, S. Coriani, P. Jørgensen, K. Kristensen, J. Olsen, and K. Ruud, Chem. Rev. 112, 543 (2012).

${ }^{77}$ K. Kristensen, P. Jørgensen, A. J. Thorvaldsen, and T. Helgaker, J. Chem. Phys. 129, 214103 (2008).

${ }^{78}$ J. F. Stanton and R. J. Bartlett, J. Chem. Phys. 98, 7029 (1993).

${ }^{79}$ P. B. Rozyczko, S. A. Perera, M. Nooijen, and R. J. Bartlett, J. Chem. Phys. 107, 6736 (1997).

${ }^{80}$ P. Rozyczko and R. J. Bartlett, J. Chem. Phys. 107, 10823 (1997),

${ }^{81}$ P. B. Rozyczko and R. J. Bartlett, J. Chem. Phys. 108, 7988 (1998).

${ }^{82}$ T. Helgaker, P. Jørgensen, and J. Olsen, Molecular Electronic-Structure Theory (Wiley, Chichester, 2000).

${ }^{83}$ S. Kvaal, J. Chem. Phys. 136, 194109 (2012).

${ }^{84}$ J. Arponen, Ann. Phys. 151, 311 (1983).

${ }^{85}$ P. Kramer and M. Saraceno, Geometry of the Time-Dependent Variational Principle (Springer, 1981).

${ }^{86}$ R. J. Bartlett, Annu. Rev. Phys. Chem. 32, 359 (1981).

${ }^{87}$ M. Nooijen, K. R. Shamasundar, and D. Mukherjee, Mol. Phys. 103, 2277 (2005).

${ }^{88}$ J. Geertsen, M. Rittby, and R. J. Bartlett, Chem. Phys. Lett. 164, 57 (1989); H. Sekino and R. J. Bartlett, Int. J. Quantum Chem. Symp. 18, 255 (1984); I. Shavitt and R. J. Bartlett, Many-Body Methods in Chemistry and Physics: MBPT and Coupled-Cluster Theory (Cambridge University Press, 2009).

${ }^{89}$ See supplementary material at http://dx.doi.org/10.1063/1.4913364 for appendices showing relations between CC-CI and EOMCC response functions.

${ }^{90}$ S. A. Kucharski and R. J. Bartlett, J. Chem. Phys. 108, 5243 (1998).

${ }^{91}$ S. A. Kucharski and R. J. Bartlett, J. Chem. Phys. 108, 9221 (1998).

${ }^{92}$ T. D. Crawford and J. F. Stanton, Int. J. Quantum Chem. 70, 601 (1998).

${ }^{93}$ J. J. Eriksen, K. Kristensen, T. Kjærgaard, P. Jørgensen, and J. Gauss, J. Chem. Phys. 140, 064108 (2014). 